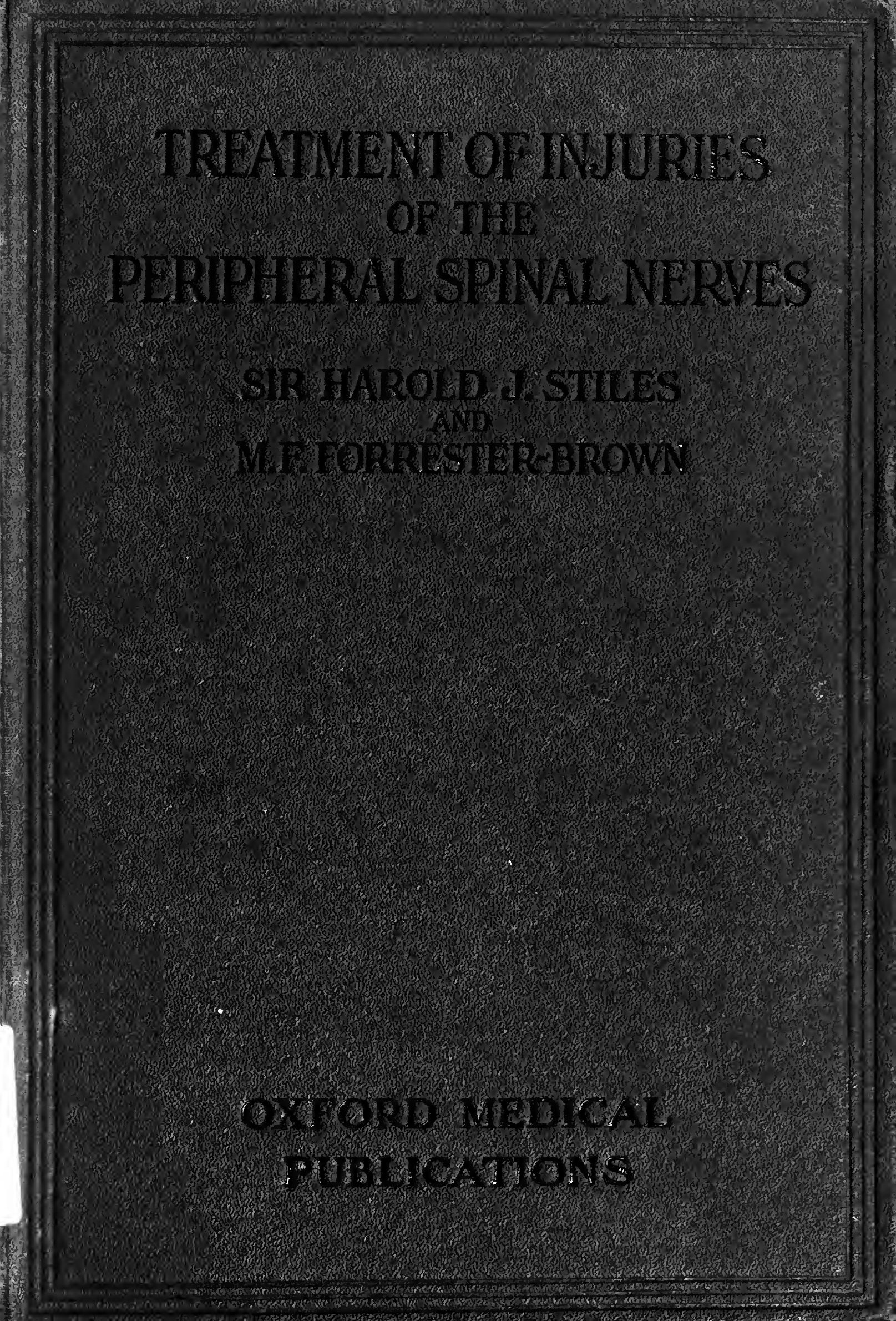




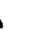




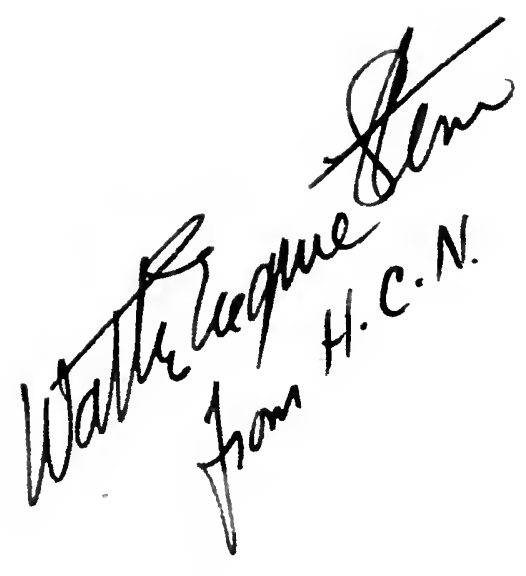




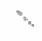

$\checkmark$ 



\section{Digitized by the Internet Archive in 2007 with funding from Microsoft Corporation}


OXFORD MEDICAL PUBLICATIONS

TREATMENT OF INJURIES OF THE PERIPHERAL SPINAL NERVES 


\title{
TREATMENT OF INJURIES
}

\author{
OF THE
}

\section{PERIPHERAL SPINAL NERVES}

BY

\begin{abstract}
SIR HAROLD J. STILES, K.B.E., F.R.C.S. (Edin.) REGIUS PROFESSOR OF CLINICAL SURGERY, UNIVERSITY OF EUINBURGH ; FORMERLY ASSISTANT INSPECTOR OF MIITAARY ORTHOPEDICS AND CHIEF SURGEON TO THE ORTHOPEIIC DEPARTMENT OF THE EDINBURGH WAR HOSPITAL, BANGOUR
\end{abstract}

AND

M. F. FORRESTER-BROWN, M.S., M.D. (LONDON) FORMERLY SURGEON, EDINBURGH WAR HOSPITAL

\section{LONDON}

HENRY FROWDE AND HODDER \& STOUGHTON

THE LANCET BUILDING

I \& 2 BEDFORD STREET, STRAND, W.C. 2 
First Printed r922

PRINTED IN ENGLAND

AT THE OXFORD UNIVERSITY PRESS

BY FREDERICK HALL 


\section{CONTENTS}

PART I

PAGES

OPERATIONS ON THE PERIPHERAL NERVES

. $I-67$

INTRODUCTORY

$$
\text { I }
$$

Anatomical Considerations

Origin of each Nerve from :

(a) Spinal roots . . . . . . . . . . . 2

(b) Plexus . . . . . . . . . . . . 3

Brachial Plexus . . . . . . . . . . 3

Musculo-spiral Nerve . . . . . . . . . . . 7

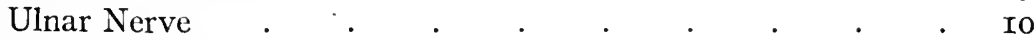

Median Nerve . . . . . . . . . . . II

Sciatic Nerve $. \quad . \quad . \quad . \quad . \quad . \quad . \quad . \quad . \quad$.

Diagnosis

(a) General principles . . . . . . . . 2I

(b) Special difficulties . . . . . . . . . . . . 30

Types of Lesions found at Operation . . . . . . 4 I

INDICATIONS FOR OPERATION . . . . . . . . . 45

Choice of Type of Operation . . . . . . 46

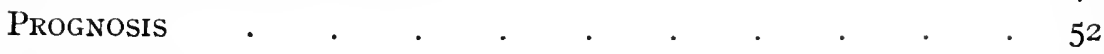

ORder of Recovery . . . . . . . . . . . 57

Treatment after Operation . . . . . . . . . . 63

PART II

OPERATIVE TREATMENT OF WAR INJURIES OF THE PERIPHERAL SPINAL NERVES . . . . . . 69-I48 General Considerations . . . . . . . . 70

Injuries to the Nerves of the Upper Extremity:

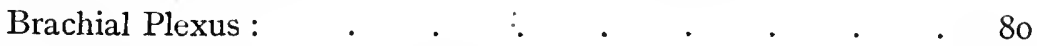

Exposure of Upper and Middle Trunks . . . . $\quad . \quad 85$

Exposure of Lowest Trunk . . . $\quad$. $\quad . \quad$. $\quad . \quad 87$

Exposure of the Entire Plexus . . . . . . 90

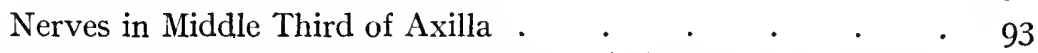

Combined Lesions of the Nerves and Vessels of the Axilla . 97

Nerves in the Lower Third of the Axilla and Proximal Half of

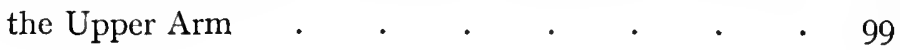


Musculo-spiral Nerve in the Lower Half of the Arm I03 Posterior Interosseous Nerve Io8 Ulnar Nerve :

In Lower Two-thirds of Forearm . . . . . $\quad$ rog

In Upper Third of Forearm, and its Transposition . . III Median Nerve :

In Lower Two-thirds of Forearm . . . . . . II7

At the Elbow and Upper Third of Forearm . . . I20

Tendon Transplantation for Median Paralysis . . . I23

Nerves of the Hand . . . . . . . . . . 124

Injuries to the Nerves of the Lower Extremity:

Sciatic Nerve : .

In Buttock and Upper Part of Thigh . . . . . $\quad$ I25

In Lower Half of Thigh ․ . . . . . . . $\quad$ I29

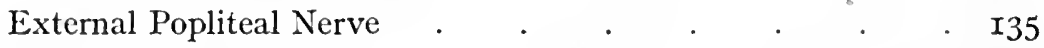

Internal Popliteal Nerve . . . . . . . . . . I36

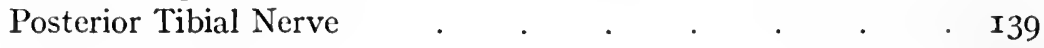

Nerves of the Foot $\quad . \quad$. $\quad . \quad$. $\quad . \quad$. $\quad . \quad$. I43

Anterior Crural Nerve . . . . . . . . . . . I44

Procedures when End-to-end Union cannot be Effected:

Nerve Grafting . . . . . . . . . . . 144

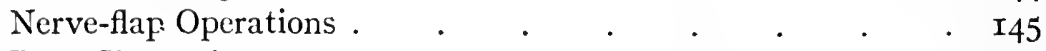

Bone Shortening . . $\quad . \quad$. $\quad . \quad$. $\quad . \quad$. 147

Treatment of Nerve Injuries when Pain is the chief Disability . $\mathbf{I}_{48}$

\section{PART III}

SURGERY OF THE PERIPHERAL NERVES . . . $149-78$

TENDON-TRansPlantations :

I. Indications . . . . . . . . . . . 149

2. Essentials for a Successful Operation . . . . I5O

3. Details of some Special Operations

(I) For Musculo-spiral Paralysis . . . . . ${ }_{56} 6$

(2) For Complete Flexor Paralysis in the Upper Limb . ${ }_{\mathbf{I} 62}$

(3) For Paralysis of the Intrinsic Muscles of the Hand - $\quad$ I66

(4) For Partial Flexor and Extensor Paralysis in the Upper

Limb . . . . . . . . . I7I

(5) For Quadriceps Paralysis and Injury . . . . I72

(6) For Paralysis of the Tibialis Anticus, or a Single Extensor I73

(7) For Complete External Popliteal Paralysis . . I75 


\section{PART I \\ OPERATIONS ON THE PERIPHERAL NERVES}

INTRODUCTORY.

Anatomical Considerations :

Origin of each Nerve from:

(a) Spinal roots.

(b) Plexus.

Distribution :

(a) Motor, including levels of branches.

(b) Sensory.

(c) Trophic.

(d) Effects of complete division.

Diagnosis :

(a) General principles

(b) Special difficulties

Types of Lesion Found at Operation.

INDICATIONS FOR OPERATION.

Choice of Type of Operation.

Prognosis.

ORDER OF RECOVERY.

Treatment after Operation.

\section{INTRODUCTORY}

In the following monograph an attempt is made to place at the disposal of the general surgeon, who may be called on to deal with an occasional case of peripheral nerve injury, the experience which has been gathered from the exceptionally abundant material provided by the Great War. It is hoped to map out for the surgeon who has no special experience of the subject those paths.which will lead to a successful result for himself and his patient and to help him to avoid those pitfalls which have entrapped most workers at first, before they learned to look out for them.

To deal successfully with a peripheral nerve lesion involves attention to several factors :

(a) First it is necessary to diagnose the existence of a lesion of the - nerve suspected, and this is not so easy as it sounds in all cases, for there 
are ischæmic conditions which simulate closely lesions of the brachial plexus and posterior tibial nerve respectively, and these are by no means improved by operative interference.

(b) Secondly it is desirable to diagnose approximately the nature and extent of the lesion, for if the respective merits of resection versus conservative methods have not been weighed in each case before operation, the surgeon will often be faced at the time by embarrassing alternatives. For example, in partial lesions of the median nerve it is often justifiable to sacrifice a few intact motor fibres in order to obtain satisfactory suture of destroyed sensory fibres, without which the hand is functionally useless. If the function of the limb has not been carefully studied before operation, an unwise decision may be made.

(c) Thirdly the operative technique of these cases is a special one, requiring an intimacy with the anatomy of the regions involved more exact than that which suffices for many other types of operation. Moreover it is important to have in mind the available methods of dealing with any given lesion, in order not to waste time over ones which are not adapted to the particular case, or which have been proved by modern experience to be doomed to failure, such for instance as the attempt to bridge gaps by foreign material of various kinds. It is now known that there is a potential gap in every nerve which can be overcome with certainty in practically all cases by a given technique, and these gaps include the ones commonly met with, where the general destruction of tissue is not so great as to make the nerve lesion itself of secondary importance.

\section{ANATOMICAL CONSIDERATIONS}

Although the difficulties of diagnosing the existence of a lesion of any given nerve are not bounded by a knowledge of the anatomy of the nerve, as the inexperienced are apt to think, yet anatomical considerations are an essential preliminary. They will therefore be dealt with first, and certain peculiarities which are apt to lead to error will be considered later.

As the external marks of injury may not always correspond exactly with the level at which the nerve has been damaged, it is important to be able to diagnose this from the clinical signs, considered in the light of a knowledge of the origin and connexions of the nerve. We will proceed then to deal in turn with each nerve, considering:

I. The Origin of the Nerve from:

(a) The Spinal Roots. These, it is to be noted, are given differently by various observers, and even within the limits of the cases tested at operation by the writers, stimulation of the same root in different cases has given different responses, suggesting that their ultimate distribution is by 
no means constant, within narrow limits. As lesions of the roots are not as amenable to operative treatment as those of the limb plexuses, it is often of importance to decide whether the injury has occurred to the former or the latter.

(b) The Limb Plexus. The arrangement of these is not uniformly constant either, but a knowledge of the common forms is valuable.

2. The Peripheral Distribution of each nerve is of even more importance in the diagnosis of the nature and extent of a lesion, but even in this variations occur, and their possibility must always be borne in mind. This matter will be dealt with at some length in a later section.

In connexion with the Motor distribution of any nerve, it is important to know the levels at which the branches leave the main trunk to supply particular muscles, both in connexion with the escape of certain muscles when a nerve has been completely divided, and also regarding the possibilities of freeing the nerve widely for suture without injuring intact branches. Attention will be drawn later to certain groups of motor branches which can be stripped up for a long distance from the main trunk in order to relax it for suture, where a big gap is present.

With regard to the Sensory distribution of the nerves, though many writers have pointed out that the areas for deep sensibility, e.g. firm pressure, overlap, so that anæsthesia of this type does not extend over the whole skin area corresponding to the nerve, yet this fact is still often overlooked, with the result that complete lesions are regarded as incomplete, or recovery is diagnosed before any has taken place.

The relations of the nerves are dealt with fully in the operative section of the book and will not be referred to in the present section, which is merely intended to remind the reader of their origin and distribution, without a mental image of which no satisfactory study of a case or operation is possible. The nerves will be dealt with now in the same order in which they appear in the operative section.

\section{BRACHIAL PLEXUS}

The nerves to the upper limb are derived from the anterior primary divisions (mixed sensory and motor fibres) of the fifth, sixth, seventh, and eighth cervical and first thoracic spinal nerves. A variable contribution is given by the first thoracic, being very small in some cases. These trunks appear in the posterior triangle of the neck, where they lie behind the scalenus anticus muscle on, or in the substance of the scalenus medius, and at the outer border of that muscle they combine to form the primary trunks of the brachial plexus. These primary trunks divide and recombine to form secondary cords, which give off the various nerves to the limb.

The relations of the brachial plexus will be dealt with fully in the operative section, p. 80. 
It may be useful here to remind the reader of the following points :

(a) Soon after emerging from the spinal foramina, the nerve roots give off the following important muscular branches:

From the fifth cervical the nerve to the rhomboids (posterior scapular).

From the fifth, sixth, and seventh cervical twigs which combine to supply the serratus magnus (long thoracic nerve of Bell).

Both these are adductors of the scapula.

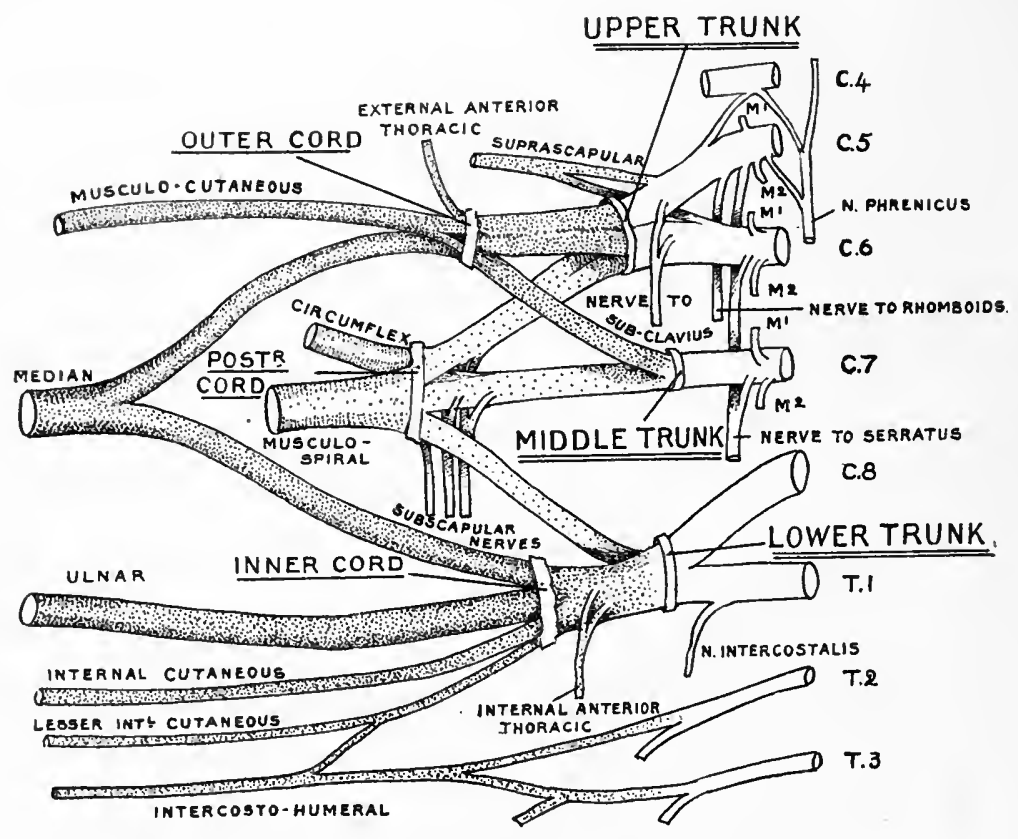

FIG. I.-The brachial plexus.

(b) The roots then form the three Primary trunks (or cords) of the plexus, thus .

Upper trunk by union of the fifth and sixth.

Middle trunk by the seventh alone.

Lower trunk by the union of the eighth cervical and first thoracic.

(c) Immediately after so combining, sometimes before it, each root divides into an Anterior and Posterior part, which form the Secondary Cords as follows:

Outer cord from the anterior parts of upper and middle trunks. Inner cord from the anterior parts of the lower trunk.

Posterior cord from all the posterior parts (usually fifth, sixth, seventh, and eighth cervical). 
(d) The Secondary cords give off the nerves to the limb as follows: Outer Cord gives off-

Musculo-cutaneous (flexors of the elbow).

Nerve to pectoralis major (external anterior thoracic).

Outer head of median (pronator teres and long flexors).

Inner Cord gives off-

Nerve to pectorals (internal anterior thoracic).

Inner head of median (pronator quadratus and thenar muscles). Ulnar nerve.

Internal cutaneous.

Posterior Cord gives off-

Circumflex (deltoid and teres minor).

Subscapulars (subscapularis, latissimus dorsi, and teres major).

Musculo-spiral (extensors of elbow, wrist and knuckles, and supinators).

Regarding the peripheral distribution of each nerve root, the following generalizations, if not regarded as infallible, may prove useful to the reader. They are derived from the statements of Benisty and correspond in most particulars with those of Kocher and of Poirier and Charpy.

Injuries of the Fifth and Sixth Cervical produce the well-known type of paralysis associated with the name of Erb, i. e.

(a) Paralysis of the abductors and external rotators of the shoulder (deltoid and supra- and infra-spinatus);

of the flexors of the elbow (biceps and supinator longus).

(b) Weakness of the adductors and internal rotators of the shoulder (teres major, pectoralis major, latissimus dorsi, and subscapularis).

(c) Very slight involvement of the pronator teres and supinator brevis; of the flexors of the wrist;

and of the thenar muscles.

If the lesion is above the point of union of the fifth and sixth, there is also paralysis of the rhomboids and of most of the serratus magnus (adductors of the scapula).

A lesion of the Outer Cord differs from that of the fifth and sixth roots in that :

(a) There is no involvement of the shoulder muscles nor supinator longus.

(b) There is complete paralysis of the pronator teres and radial flexors of wrist.

2. A lesion of the Seventh Cervical causes paralysis of :

(a) The coraco-brachialis (which is not involved with the biceps in Erb's).

(b) All the musculo-spiral distribution except the supinator longus. 
In contrast with this the Posterior Cord gives the additional paralysis of :

(a) The circumflex nerve (deltoid and teres major).

(b) The subscapular nerves (subscapularis, teres major, and latissimus dorsi), and also supinator longus.

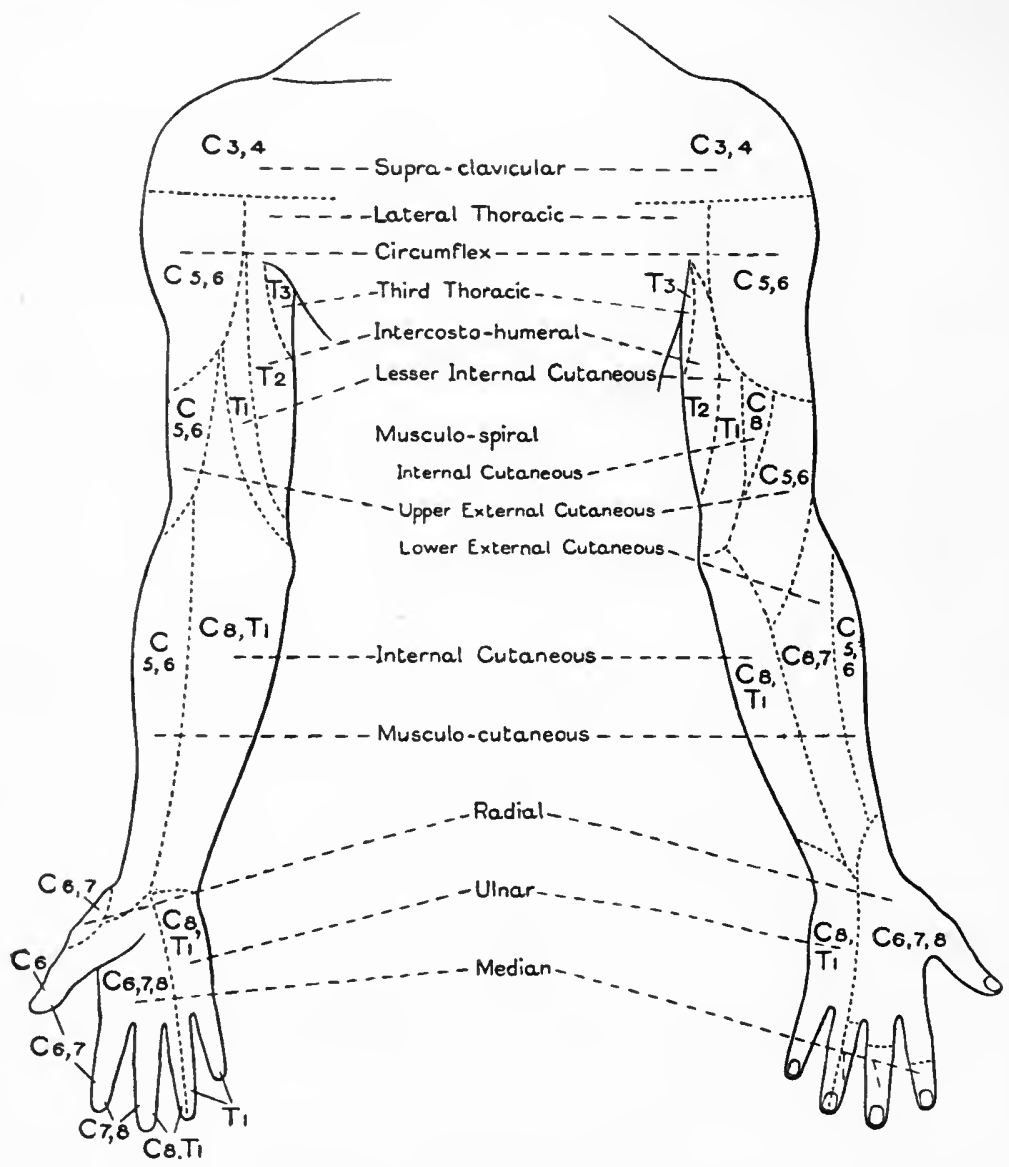

FIG. 2.-Innervation of the skin of the upper limb. All these areas of distribution are the maximum areas.

3. A lesion of the Eighth Cervical and First Thoracic causes paralysis 'of :

(a) The intrinsic muscles of the hand.

(b) Flexors of the digits.

In contrast with this, lesions of the Inner Cord also involve the flexor carpi ulnaris, and combined lesions of the Median and Ulnar affect in addition :

(a) The pronators.

(b) All the wrist flexors. 
The Sensory distribution of each root is shown in the accompanying diagram (Fig. 2).

We pass now to a consideration of the individual nerves which spring from the brachial plexus.

The nerves to the shoulder girdle and shoulder muscles (the posterior scapular, subscapulars, circumflex, and thoracics) run so short a course before breaking up into fine branches that they are seldom amenable to surgical repair, apart from neurolysis, and therefore they will not be considered in any further detail. Fortunately they are seldom all divided in the same case, while the muscles which they supply are usually able to substitute for one another to a considerable degree. Where the shoulder muscles are so extensively injured as to leave the joint flail, the best treatment is an arthrodesis, as movements of the scapula on the trunk give very useful function for the limb.

The nerves which run a long course down the limb are those which are suitable for suture, in spite of the existence of gaps of several inches, provided a careful technique is carried out in accordance with anatomical considerations. These will now be considered in detail.

\section{MUSCULO-SPIRAL}

\section{Origin.}

(a) From roots: sixth, seventh, and eighth cervical, chiefly from the seventh, occasionally from the first thoracic also (Poirier and Charpy).

(b) From the plexus-posterior cord, below the origin of the subscapulars, in common with the circumflex; it practically forms the continuation of the cord into the limb

\section{Branches.}

(I) Internal cutaneous.

(2) To triceps: a leash of branches arises, of which the highest, to the Long Head, leaves the main nerve on the latissimus dorsi tendon, and one to the Inner Head comes off next and accompanies the ulnar nerve.

The others to the inner and outer heads and anconeus appear to come off in the musculo-spiral groove, but can easily be freed up to the apex of the axilla.

(3) External cutaneous also comes off in middle of arm, and runs parallel to the nerve for some distance in a more superficial plane, so that it forms a useful guide.

(b) In arm.

(a) In axilla. 
(4) To brachialis and supinator longus in the groove between those two muscles at the level of the elbow-joint.

(5) To extensor carpi radialis longior (and sometimes to the brevior also) just below the abovenamed.

$\left.\begin{array}{l}\text { (6) Radial, the sensory terminal branch. } \\ \text { (7) Posterior interosseus, the motor terminal }\end{array}\right\}$ one.

(c) At elbow.

(d) At neck of radius

This nerve before entering the substance of supinator brevis gives off twigs for that muscle and for the extensor carpi radialis brevior.

In the supinator brevis it breaks up into a leash, of which the superficial set supply:

Extensor communis digitorum and minimi digiti,

Extensor carpi ulnaris;

while the deep set supply:

Extensores ossis metacarpi and brevis pollicis.

Extensor indicis.

Extensor longus pollicis.

The Joints supplied by the musculo-spiral are:

Elbow (by ulnar collateral, radial and muscular branches).

Superior radio-ulnar.

Carpus (by posterior interosseus).

Metacarpo-phalangeal joints, a variable number.

These latter show trophic changes in certain cases of injury to the musculo-spiral nerve, especially in irritative lesions, but they are normal in many other cases of complete division of the nerve.

\section{Musculo-Spiral Distribution.}

Complete division of the musculo-spiral before it has given off any branches results in:

Paralysis of:

Extensors of elbow, wrist, knuckles and all joints of thumb.

Supinators (including supinator longus, which is chiefly a flexor of the elbow, but excluding the biceps, which is a powerful supinator).

\section{Anæsthesia :}

Cutaneous (to wool and prick) over the dorsum of the second and third metacarpals and corresponding proximal phalanges. At its maximum this extends over the whole dorsum of thumb and almost to the tips of index and middle fingers; at its minimum it is reduced to the index knuckle. The areas of the internal and external cutaneous branches often 
fail to show anæsthesia, even in the presence of high lesions, owing to overlapping by the internal cutaneous and musculo-cutaneous nerves.

Deep anæsthesia exists as loss of muscle-pain in the above-mentioned muscles. Joint-sense is rarely affected.
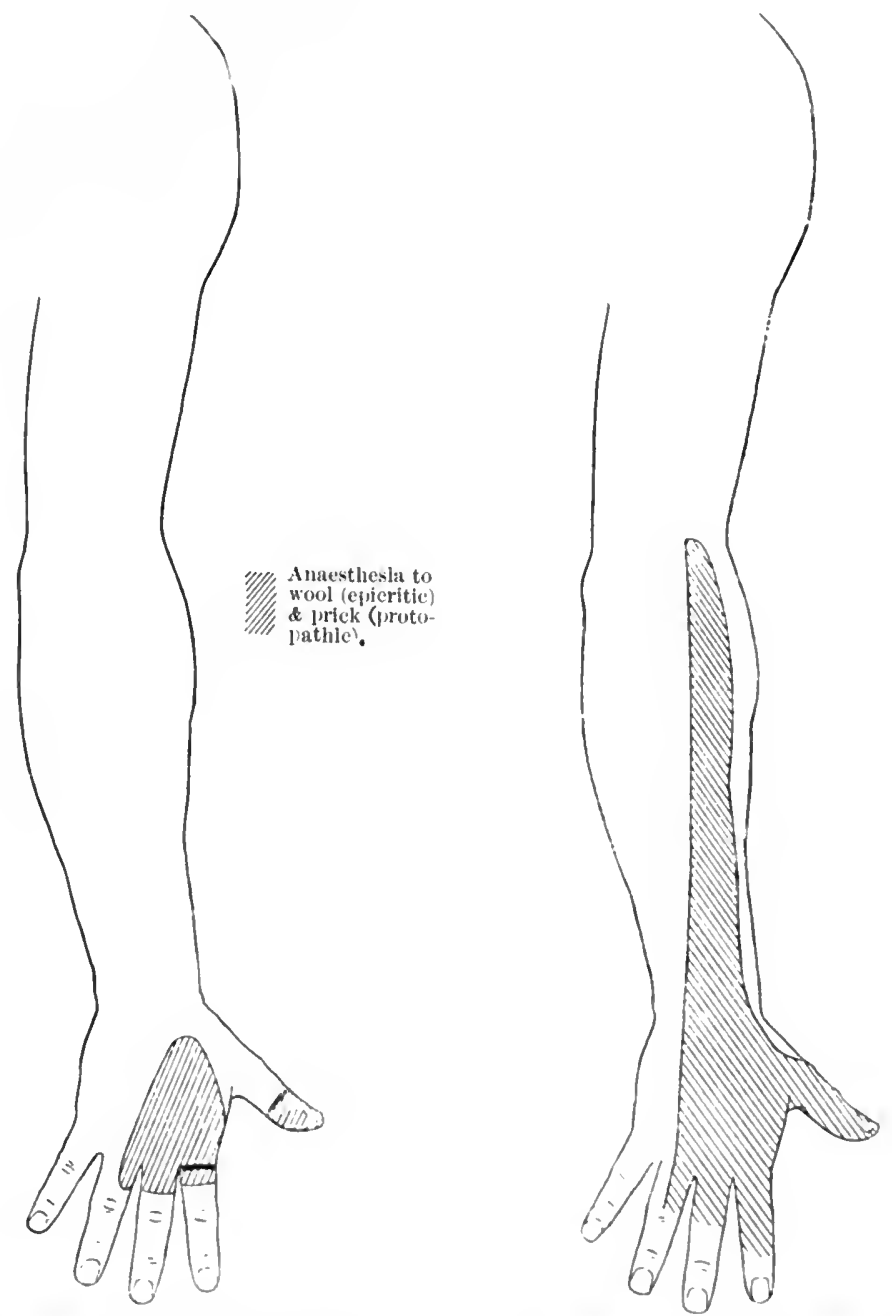

FIG. 3a.-Musculo-spiral (complete division). Dorsum.

Fig. 3b.-Musculo-spiral. Maximum area of anæsthesia (not found in all cases).

Trophic changes may occur in the form of a dry arthritis of the knuckles with limitation of flexion. Changes in the skin and nails are uncommon. 


\section{ULNAR NERVE}

\section{Origin.}

(a) From roots: eighth cervical and first thoracic, and sometimes seventh cervical (Poirier and Charpy).

(b) From plexus : inner cord, below origin of internal cutaneous.

\section{Branches.}

No branches arise in the arm, which makes it easy to free a long stretch of the nerve. Occasionally there is a communicating branch to median in middle of arm.

\section{Motor.}

(I) One to each head of the flexor carpi ulnaris arises behind the internal condyle; they can be traced up from the main nerve to lower third of arm.

(2) The flexor profundus digitorum to ring and little fingers receives branches on its anterior surface:

(a) just below those to the ulnaris (traceable up into arm);

(b) in middle of forearm.

(3) The intrinsic muscles of the hand are supplied by the two terminal branches of the ulnar into which it divides opposite the pisiform:

(a) Superficial branch to palmaris brevis (also sensory twigs).

(b) Deep branch to:

Abductor opponens and flexor brevis minimi digiti;

All the interossei;

Third and fourth lumbricals;

Adductor pollicis and deep head of flexor brevis.

\section{Sensory.}

(I) Palmar cutaneous arising 2 in. above wrist (or higher from a special vaso-motor branch).

(2) Dorsal cutaneous, at same level, but turning backward round the ulna, so that it is not relaxed by flexion of the wrist, and therefore it limits the amount that a distal segment of ulnar can be pulled upward for the purpose of suture.

(3) Digital, arising in the palm from the superficial terminal branch supplying the little finger and adjacent side of ring finger.

The Joints supplied by the ulnar are :

Elbow.

Inferior radio-ulnar.

Carpal.

Two inner knuckles.

Interphalangeal joints of ring, little and middle fingers. 


\section{Distribution.}

Complete division of the ulnar results in :

Paralysis of-Ulnar flexor of wrist;

Flexors of ends of ring and little fingers (Fig. $4 d$ );

Adductors of all digits and abductors of the four fingers; therefore loss of power to grip firmly with the thumb and index (Fig. 4a);

Flexors of all knuckles and extensors of interphalangeal joints, i. e. interossei and two inner lumbricals, therefore clawing, most marked in little finger, progressively less in ring and middle fingers, due to unopposed action of sublimis and extensor communis (Figs. $4 b$ and $c$ ).

Anæsthesia (Figs. $5 a$ and $b$, p. I4) :

(a) Deep-to muscle pain of all the above muscles;

to joint sense of three joints of little finger and two interphalangeal joints of ring finger, occasionally fewer ;

to pressure and deep pain of ulnar border of hand and whole of little finger (occasionally only of tip of little finger).

(b) Cutaneous (wool, prick, and temperature).

Ulnar half of hand as far as second space, whole of little finger and ulnar half of ring finger.

Trophic changes in the corresponding muscles, joints and skin. Especially noticeable are atrophy of the pulp and redness of end of little finger (Fig. $4 d$ ).

\section{Origin.}

\section{MEDIAN NERVE}

(a) From roots: sixth and seventh cervical (outer head); and eighth cervical and first thoracic (inner head).

(b) From plexus: outer cord to outer head (to pronators and flexors).

Inner cord to inner head (to intrinsic muscles and skin).

\section{Branches.}

None in arm, except an occasional communication with the ulnar and, fairly commonly, a low separation of the musculo-cutaneous or a part of it from the outer head of median.

\section{Motor.}

(I) To pronator teres two branches from outer side of nerve to superficial head of muscle

(2) To superficial flexors in forearm, a leash of branches, which can be freed up from median as high as elbow.

i. e. to deep head of pronator teres,

At level of internal condyle.

flexor carpi radialis and palmaris longus, flexor sublimis digitorum.

Between two heads of pronator teres. 


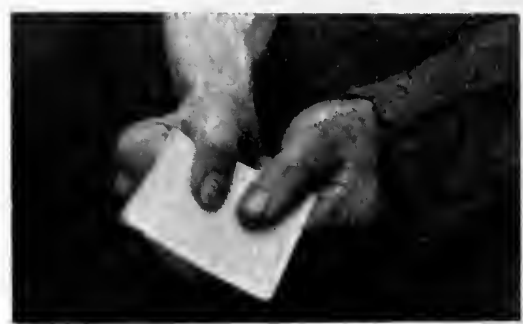

FIG. 4a.-Paralysis of $R$. adductor pollicis; L. thumb normal. Compare grasp on the two sides; that of $\mathrm{R}$. depends on the flexor longus and opponens and is very weak; on $\mathrm{L}$. side the adductor pollicis is acting strongly and keeps thumb extended.

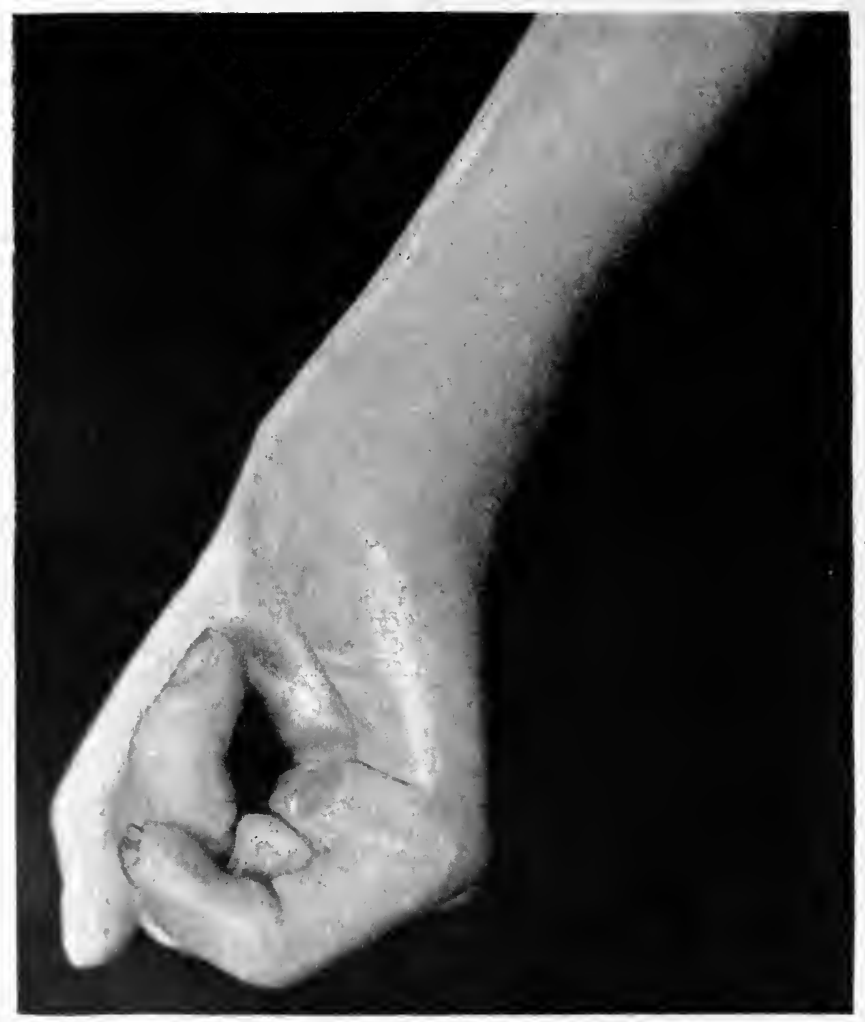

FIG. 4 d.-Trophic changes most marked in the little finger, owing to ulnar nerve injury. Note the condition of the nail. Incomplete flexion from paralysis of the profundus. 

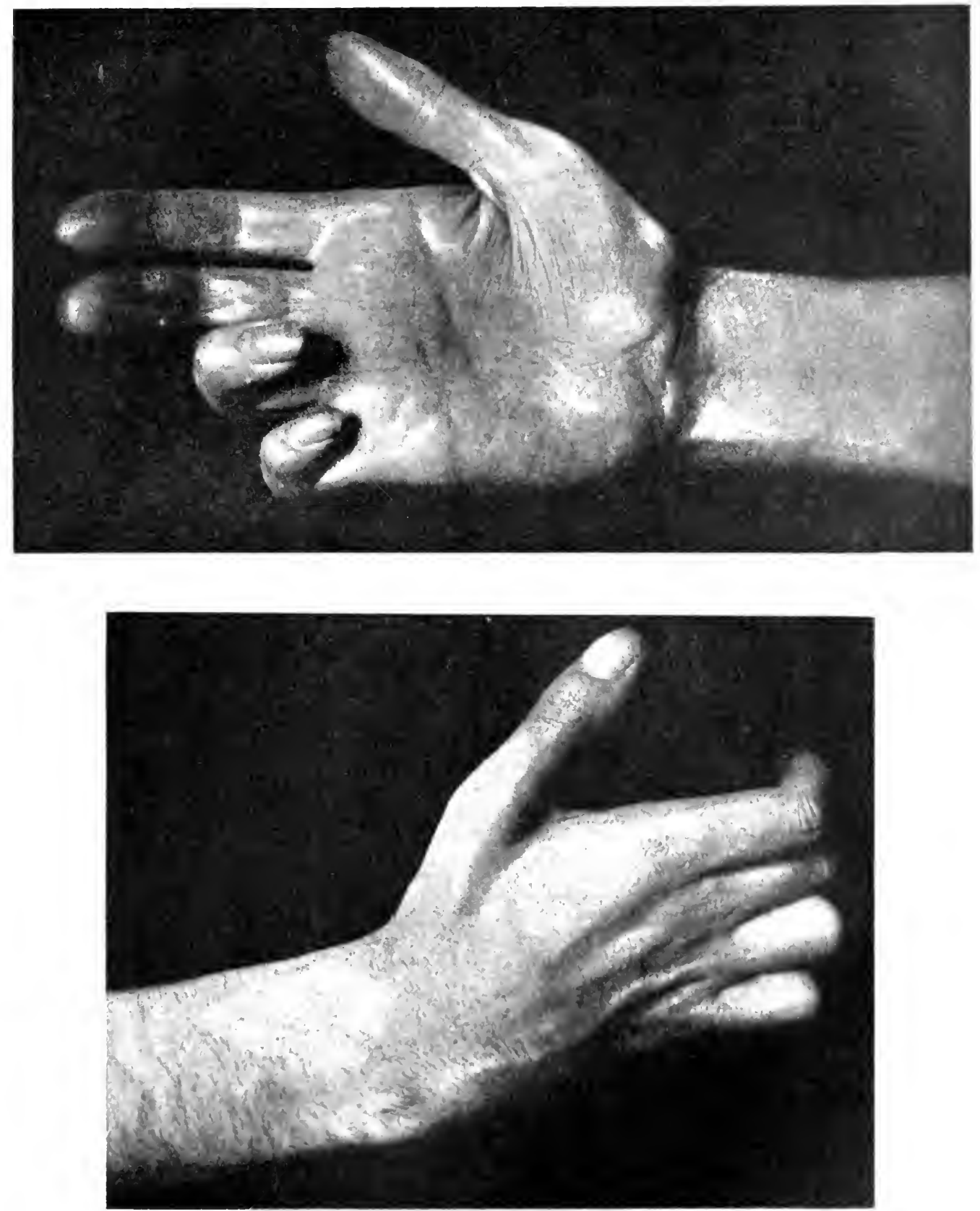

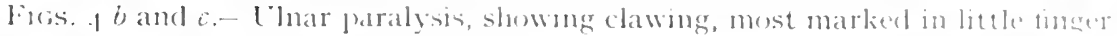
also a little contracture in flexion. Note atrophy in the interesseus apilces, esperialls first; also curving of the mats of two imner fingers. 

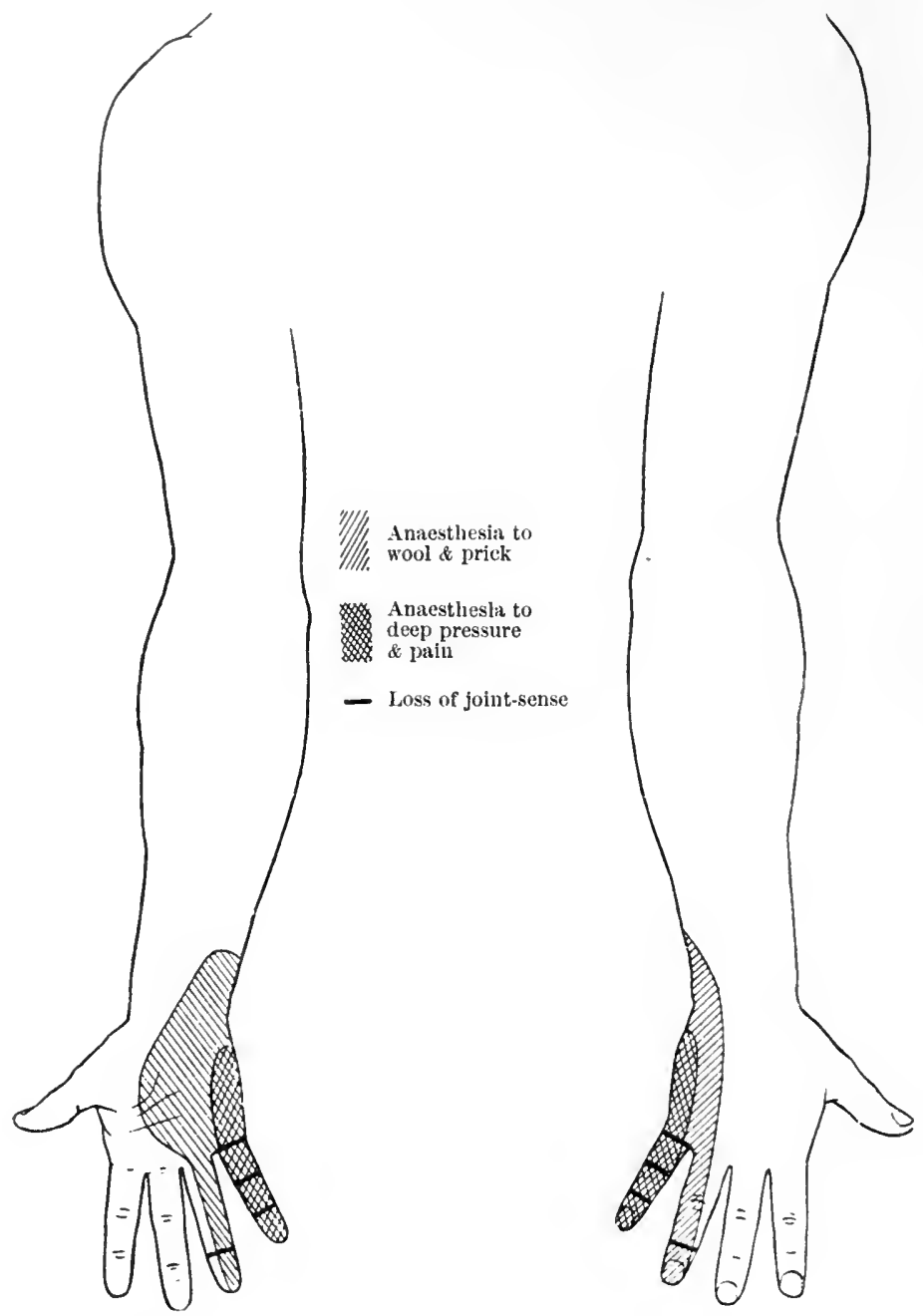

FIG. 5a.-Ulnar (complete division). FIG. 5 b.-Ulnar (complete division). Palm. Dorsum.

(3) Similar leash to deep flexors (anterior interosseus),

i. e. to flexor profundus to index and mid fingers, flexor longus pollicis, pronator quadratus (entering its deep aspect).

(4) A few twigs to flexor sublimis (radial and coronoid heads) coming off in middle of forearm.

(5) To thenar muscles. 
Thenar branches of median.

External to abductor, opponens, flexor brevis pollicis and first lumbrical.

Internal to second lumbrical (with digital branch).

Sensory branches.

(I) Palmar, coming off $x \frac{1}{2}$ in. above wrist.

(2) Digital-external coming off with the motor branch and going to thumb and radial side of index,

internal to second and third spaces and corresponding sides of fingers.

Joints supplied by median are :

Elbow (by branch to pronator teres and anterior interosseus recurrent).

Wrist and carpus (by anterior interosseus).

Inferior radio-ulnar (ditto).

Knuckles.

Interphalangeal of thumb and index, middle and ring fingers.

\section{Distribution.}

Complete division of median results in :

Paralysis of :

Pronators.

Radial flexors of wrist.

Flexors of all proximal interphalangeal joints.

" " terminal joints of thumb, index and middle fingers.

" . first, second, and third metacarpo-phalangeal joints.

Extensors of interphalangeal joints of outer three digits (lumbricals).

Abductor and opponens pollicis, i. e. loss of the power to rotate thumb opposite index finger for the pincer action in picking up objects.

Anæsthesia (Figs. $6 a$ and $b$ ):

Deep : to muscle-pain of the above muscles.

Anæsthesia to pressure of ends of thumb, index and middle fingers.

Loss of joint sense in interphalangeal joints of index and terminal joints of thumb and middle fingers (occasionally more).

Cutaneous: Front of index, middle and radial half of ring fingers ; also dorsum of terminal one and a half phalanges of these; also radial half of palm, excluding part of thenar eminence, which is supplied by musculo-cutancous.

Trophic changes are marked in cases of injury to the median nerve, being most advanced in the terminal phalanx of the index finger, and less so in those of the middle finger and thumb, which sometimes escape entirely. 

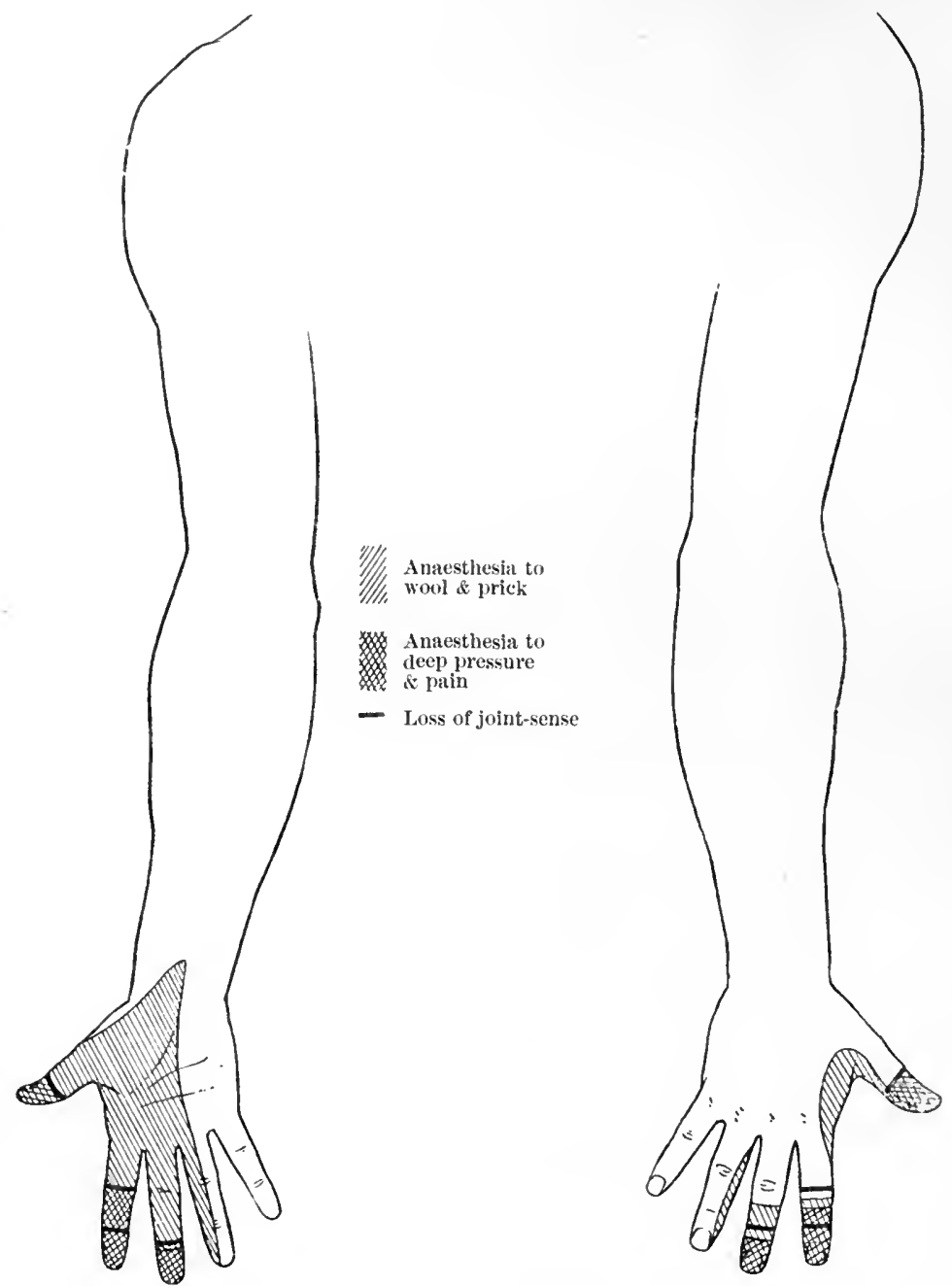

FIG. 6a.-Median (complete division). Palm.

FIG. 6b.-Median (complete division). Dorsum.

The changes vary in different cases, depending partly on whether the lesion is complete or partial, and also on the extent to which the arterial supply to the limb has been interfered with.

Cases of complete division tend to show:

atrophy of the pulp of the digits ;

curving of the nails, both longitudinally and transversely; and cracking ;

a slightly red, shiny appearance of the skin over the anæsthetic area, where it is atrophic and has lost its hairs and glands, and is 
consequently dry, compared with that in neighbouring healthy parts ;

marked atrophy of the muscles on the outer half of the thenar eminence, exposing the metacarpal; also of the outer half of the flexor aspect of the forearm, so that the interosseus membrane can be palpated.

Cases where the main artery has been obliterated also, show atrophy of the whole hand, which appears like that of some smaller person, with tapered fingers and pale, smooth skin, even though its fellow may be the horny hand of a manual labourer.

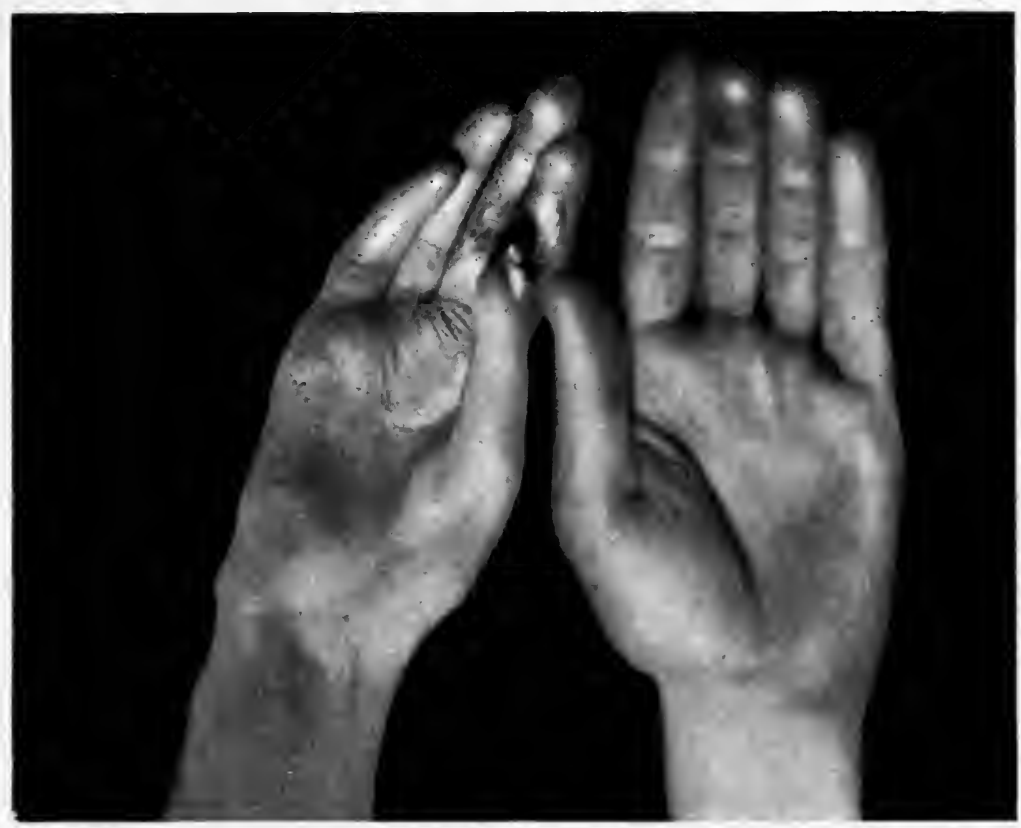

Fig. 7.-After ligature of the brachial artery, atrophy of the whole hand, shown beside the normal one; skin atrophic, but covered with scales; fingers tapered; muscles wasted

Cases with irritation of the median frequently have a succulent appearance of the digits, which are purplish-red at the tip and may have a little heap of cuticle under the free-edge of the nail ; the nails tend to be thick and cracked, sometimes looking as if they had been half wrenched out of their bed; the skin is shiny and may sweat excessively, when there is apt to be a papular sweat-rash, or it may be dry and covered with fine scales.

All intermediate variations between the two types may be met with. In many cases a whole nail falls off and is only slowly replaced. Commencing recovery can often be diagnosed by noticing improved nutrition in the base of the nail. 


\section{SCIATIC}

Origin. From the anterior primary divisions of the fourth and fifth lumbar and first, second and third sacral ; occasionally fourth sacral also (Poirier and Charpy).

The nerve arises in two parts: (a) the peroneal, which becomes the external popliteal, highest and from posterior secondary divisions of the fourth and fifth lumbar and first and second sacral.

(b) The tibial, which becomes the internal popliteal and hamstring nerve, lower and from anterior secondary divisions of the fourth and fifth lumbar and first, second and third sacral. The hamstring nerve is often independent right up to its origin; at other times its branches run in the main sciatic sheath and appear to be given off from the main nerve at various levels in the thigh.

The level down to which the peroneal and tibial nerves are incorporated in a common sheath also varies; they are sometimes separate throughout their course, often so from the level of the notch downwards, and occasionally they are bound together closely as low as the popliteal space. Usually they can be easily separated in the middle of the thigh, and always they can be freed from one another up to the notch by an incision in the sheath.

\section{Branches in the Buttock.}

Hamstring nerve from the tibial (internal) nerve, forming :

(I) Superior nerve to semitendinosus;

(2) Nerve to long head of biceps, which runs superficially across sciatic.

(3) Inferior nerve to semitendinosus, which may come off in middle of thigh.

(4) One or two nerves to semimembranosus and one to adductor magnus.

\section{Branches in Thigh.}

(Parts of hamstring nerve appearing to come from tibial nerve, as noted above.)

Nerve to short head of biceps-from peroneal (external) nerve.

Superior articular nerve to knee-, , , ,

Terminal branches: external and internal popliteal.

\section{Branches of External Popliteal Nerve.}

(a) In popliteal space:

Nervus communicans fibularis (sensory, forming part of ext. saphenous).

Inferior articular to knee. 
(b) At neck of fibula the nerve breaks up into three:

(I) Nerve to tibialis anticus (recurrent tibial), which sometimes gives a twig to peroneus longus.

(2) Musculo-cutaneous, which supplies the peroneus longus and brevis as it passes between them, and then becoming subcutaneous in the lower third of the leg in the line of the septum between the extensors and peronei, is distributed by two branches:

(a) Internal to inner side of great toe and first cleft with corresponding sides of toes.

(b) External to third and fourth clefts and corresponding toes.

(3) Anterior tibial, which furnishes the following branches:

(a) Motor to: Tibialis anticus by upper and lower twigs ;

Extensor longus digitorum;

Extensor propius hallucis;

Extensor brevis digitorum.

(b) Sensory to first cleft and corresponding sides of toes.

\section{Branches of Internal Popliteal.}

(r) Sensory. Nervus communicans tibialis, which helps to form external saphenous supplying outer side of leg, foot and little toe.

(2) Motor to:

Gastrocnemii, the branch to inner head coming off highest;

Soleus, coming off sometimes with branch to outer gastrocnemius, and running on the superficial aspect of the muscle, between whose two heads it can be traced down to the middle of the leg, giving off branches to each as it goes, and ending in the inner head.

Plantaris.

Popliteus, running over the posterior surface of the muscle and turning up its distal border to enter the deep aspect. This branch may supply the tibialis posticus, thus explaining the escape of that muscle in some complete lesions of the posterior tibial nerve.

(3) Articular branches to the knee-joint-two or three.

(4) Terminal branch, the posterior tibial.

\section{Branches of Posterior Tibial Nerve.}

(i) Motor to:

Tibialis posticus, arising above the arch of the soleus usually;

Flexor longus hallucis, running along with the peroneal vessels to the deep aspect of the muscle.

Flexor longus digitorum, some twigs high up and some near ankle. 
(2) Sensory :

Internal malleolar.

Internal calcanean.

(3) Terminal, in the hollow behind internal malleolus : internal and external plantar.

Branches of Internal Plantar (corresponding to median in hand).

Motor to :

Abductor hallucis, one for calcanean head arising before the nerve passes between the two heads; others as it does so.

Flexor brevis hallucis; in common with cutaneous branch to inner side of great toe.

Flexor brevis digitorum, entering its superior (deep) surface.

Accessorius, entering its inferior (superficial) surface.

First lumbrical, from cutaneous branch to first cleft.

Sensory: cutaneous twigs to sole, along cleft between internal and central parts of plantar fascia.

Three digital branches to inner side of great toe and first and second clefts.

Branches of External Plantar (corresponding to ulnar in hand).

Motor to :

Abductor minimi digiti.

Flexor brevis minimi digiti by the cutaneous branch to outer side of little toe.

Second, third and fourth lumbricals, that to second from nerve to adductor hallucis.

Adductor hallucis.

All the interossei.

Sensory to :

Outer side of little toe, communicating with external saphenous.

Fourth cleft and corresponding sides of toes.

\section{Joints supplied by Sciatic.}

Hip (by the hamstring nerve).

Knee.

Ankle.

Both tibio-fibular joints.

Tarsal and all toe-joints.

\section{Distribution of Sciatic.}

Complete division of the sciatic at its origin results in :

Paralysis of all hamstrings, i.e. all flexors of knee;

all leg and foot muscles. (Foot drops from effect of gravity.) 
Anæsthesia (Figs. $8 a$ and $b$, p. 22).

(a) Deep :

Loss of joint sense in ankle and all foot and toe joints.

Loss to pressure distal to the necks of the metatarsals.

Loss of muscle-pain in all the paralysed muscles.

(b) Epicritic and protopathic (cutaneous) over:

(i) all leg except a strip down inner side (long saphenous from anterior crural) ;

(ii) all foot except under the arch (long saphenous from anterior crural).

Trophic : wasting of all the paralysed muscles ;

clawing of the toes, especially where posterior tibial alone, or chiefly affected (Fig. $8 \mathrm{c}$ ) ;

adhesions in all the joints below the ankle;

shiny skin with loss of hair and absence of sweating;

trophic ulcers, especially on points of pressure (heel and balls of toes).

\section{DIAGNOSIS}

\section{a. GENERAL PRINCIPLES}

The diagnosis of a nerve injury requires a careful examination of the various functions of the nerve, motor, sensory and trophic, and also the electrical responses. As with diagnosis in many other branches of Surgery, error can only be avoided by a wide exposure of the part affected. In the case of the peripheral nerves the whole limb and adjacent part of the trunk must be bared. A case which illustrates this was seen by the writers, in which there was a complete paralysis of all the forearm and hand muscles following a gunshot wound just above the elbow. There was a large scar over the origin of the supinator longus, which could easily have been the site of a complete division of the musculo-spiral, but was not extensive enough to involve the median and ulnar, and there was no exit scar because the missile had been removed through the entry wound. Examination of the limb higher up revealed the mark of a pressure sore, just below the anterior axillary fold, which the man then explained had been caused by the ring of a Thomas' splint. In view of this, no operation was resorted to then, and complete spontaneous recovery occurred.

The following routine examination has been found to give all essential information without being unduly time-taking, and a chart which was in use at the Edinburgh War Hospital is appended (p. 24). After a little practice it can be filled up in 3-5 minutes, provided the patient is intelligent.

I. Examination of all scars. Even if the line joining the entry and exit scar does not cross the course of a nerve trunk, it must be remembered 

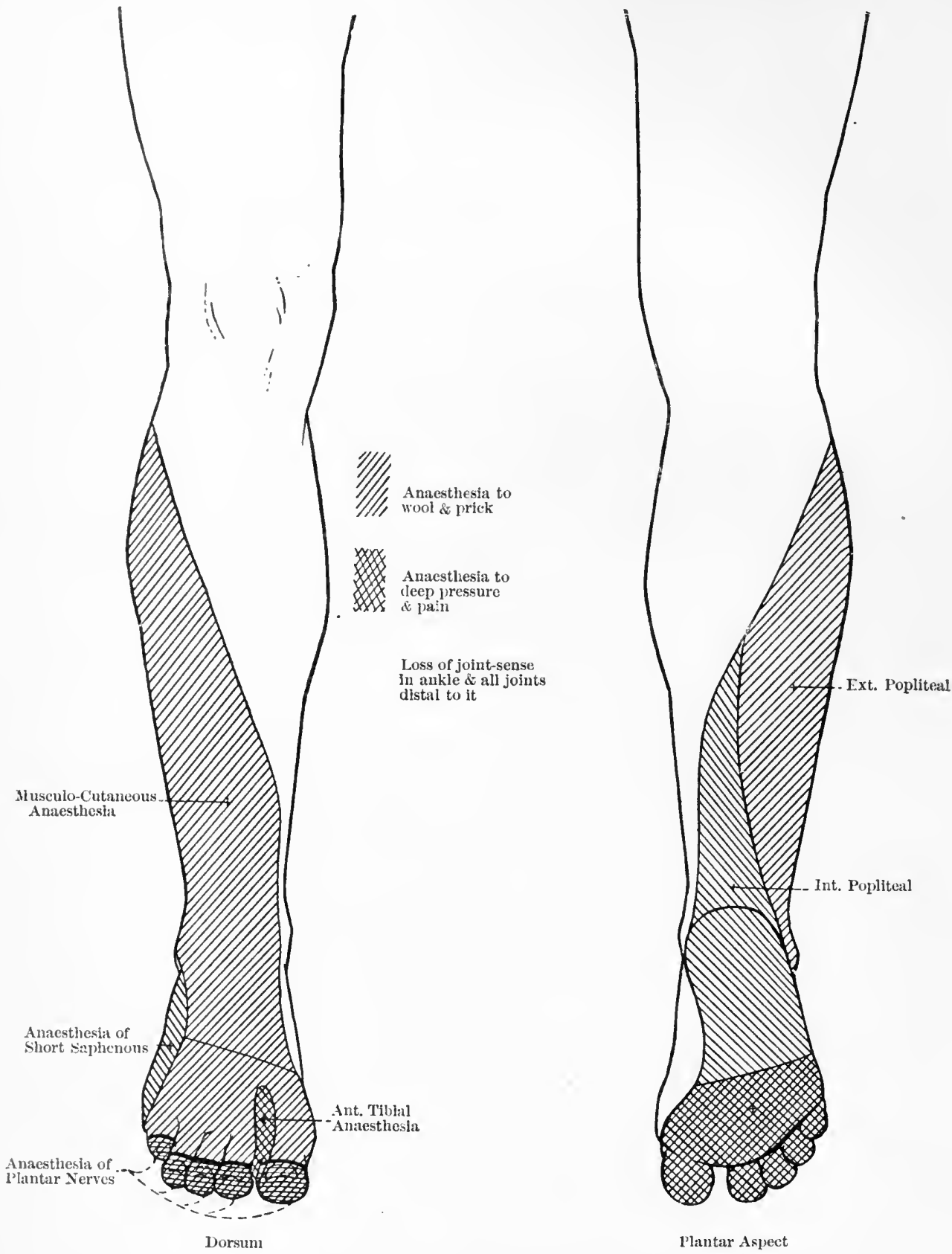

FIGS. $8 a$ and 8 b.-Anæsthesia in a case of complete division of the Great Sciatic nerve. 
that the missile may in its course have ricochetted off a bone, or a fragment of it may still remain inside.

2. Examination of the bones for fracture, as callus may have involved the nerve secondarily, or a fragment of bone may have done so ; moreover an unnoticed non-union of a bone may be the cause of the muscular weakness, of which the patient complains.

3. Examination of the joints by passive movements to ascertain that the paralysis is not of mechanical origin.

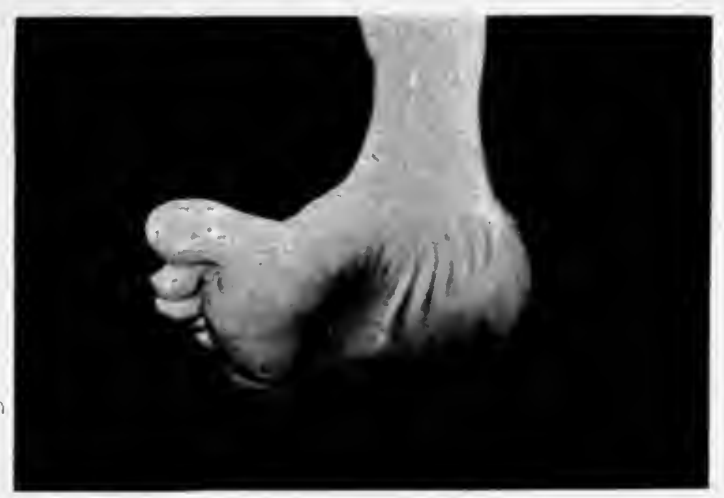

FIG. 8c.-Claw-foot in sciatic, or posterior tibial paralysis; some equinus, and trophic sore under head of fourth metatarsal.

4. Testing the active movements of each muscle below the level of the injury.

\section{Motor Function.}

In testing active movements, the following precautions must be observed :

(i) The joint above that on which the muscle acts must be fixed passively by the observer, otherwise small movements of the joint may be missed, or may be falsely induced. Thus in testing the action of the flexor profundus digitorum on the terminal phalanges, the middle and proximal phalanges must be firmly grasped by the observer and fixed ; otherwise the contraction of the sublimis, while bringing down the middle phalanges, may jar the distal ones and simulate a weak active flexion ; this is the more marked when some contracture of the profundus already exists.

(ii) If no obvious active movement is obtained, it is then necessary to place the limb in the neutral position as regards gravity, as that may be too powerful a resistance for a weakened muscle. For instance, when the musculo-spiral is recovering after a nerve suture, it is found at first that the carpal extensors are unable to dorsiflex the wrist against gravity (in 
full pronation), whereas they are able to do so in mid-supination, or at a still earlier stage, in full supination only, as in this latter they are aided by gravity. This contraction, assisted by the force of gravity, must not be confused with the rebound from active palmar flexion, which does not represent any activity of the extensors at all, though it is often mistaken for that.

(iii) If no active movement is obtained by the above methods, then it is necessary to feel the belly and the tendon of the suspected muscle and also to observe them in a good light, when very weak contractions can usually be detected. Here also fallacies are possible, for in the presence of paralysis, neighbouring healthy muscles are usually thrown into contraction by the patient's effort to obtain the required movement, and these are apt to lift the paralysed muscle forward, or if they are its antagonists, to put its tendon on the stretch, simulating a contraction of the muscle itself. Indeed, during recovery from paralysis, there is no definite line between this passive stretching of the paralysed muscle, and the recovery of its tone which makes its tendon more tense during the stretching, and which precedes any actual contraction of the muscle itself. The observer who watches a case week by week, may feel certain that recovery has begun and yet be unable to convince an onlooker; yet one or two weeks later there may be sufficient evidence to satisfy the most sceptical.

5. Sensory Tests. Alterations of sensation usually give more reliable information as to the extent of the nerve injury than do those of motor function, for the available tests are more delicate.

Some workers have used a most elaborate system of sensory tests, but these are not essential in the majority of cases of peripheral nerve injury. The following have been found sufficient to allow a correct pre-operative diagnosis in the majority of many hundreds of cases; while there are instances where complete loss of function of a nerve exists when the fibres have no interruption of their continuity, or others where an abnormal nerve supply to a muscle leads to the mistaken diagnosis of incomplete lesion, and in such cases no tests, however elaborate, will prevent error.

(a) The first test is for Tinel's sign of tenderness along the course of the nerve with a tingling sensation, referred to the peripheral distribution of the nerve. The lowest level of tenderness is supposed to represent that reached by the new axones as they grow down after an injury or suture.

\section{SENSORY CHART}

Subjective Disturbances (pain, tingling, burning, \&c.).

Distribution.

Character.

Date of onset.

Progress. 
Level of Tenderness on Nerve.

Distribution of referred pain.

Distribution of Ancesthesia to :

Wool.

Prick.

Pressure.

Joint sense.

Muscle tenderness.

Distribution of Hypercesthesia of:

Skin.

Joints.

Muscles.

Reflexes.

Superficial.

Tendon jerks.

\section{TROPHIC CHANGES}

Distribution of Skin Changes.

Sweating.

Scaliness.

Rash.

Ulcers.

Nail Changes.

Vaso-motor Phenomena.

Character of pulse.

Congestion.

Pallor.

Swelling.

CEdema.

Muscle Changes.

Wasting.

Contractures.

Joint Changes, including $X$-ray appearances.

This sign, therefore, is valuable for prognosis. If it is found below the original wound, it proves that the nerve is not completely cut through ; as, however, there is nothing quantitative about it, it does not prove that the majority of fibres are not interrupted and irrecoverable. Where a large neuroma can be felt at the level of the injury, while Tinel's sign is entirely absent below, a complete division of the nerve can be diagnosed with certainty.

Where this tenderness extends up the nerve above the wound, as it is apt to do in cases of irritation of the nerve with pain, it shows that the pathological process has ascended up the nerve, and therefore, no operative procedure which does not deal with the nerve above the level of tenderness, is likely to benefit the pain.

(b) Next Epicritic sensation is tested by touching the suspected area with 
a wisp 'of cotton wool or a camel's hair brush. In doing this it is essential that the patient's eyes should be shut, otherwise visual impressions will be interpreted by the patient as tactile ones. Moreover, a patient who is unwilling to have an operation, or unduly optimistic after one, may give misleading replies.

Testing the appreciation of the finer degrees of temperature has not the imporfance in cases of peripheral nerve injury that it has in ones of a central origin, for unlike the latter, peripheral cases tend to have it involved together with the tactile.

(c) Propathic Sensation (superficial pain) is tested with the point of a pin, and this should be held so that the pad of the observer's finger touches the patient simultaneously with the pin, otherwise he may mistake pressure by the pin for the pain sense which it should evoke. As pressure is recorded by a different set of nerve endings, this mistake may lead to errors of diagnosis, besides much waste of time. If the finger pad is applied occasionally without the pin, most mistakes will be eliminated, including deliberate ones on the part of the patient.

(d) Deep sensation, which is said to be served by nerve-fibres running with the tendons, is tested as follows:

(I) Muscle-pain by pinching the superficial muscles supplied by the nerve and asking the patient to compare the sensation with that evoked in corresponding muscles of the healthy limb. The pain is increased in cases of irritation of the nerve and during regeneration as soon as new axones have reached the muscle, so that from this test, the probability of speedy return of power in a paralysed muscle can be foretold. Next to Tinel's sign, this is the most valuable for prognosis and for distinguishing complete from incomplete lesions.

(2) Pressure sensation is tested by pinching the soft parts and bone, or applying firm pressure with the blunt end of a pin to the most peripheral part of the nerve distribution and thence working centrally. In testing all tactile sense it is important to work from the anæsthetic area towards that of normal sensation, as the first appreciable stimulus is more readily noted by the patient than is the disappearance of one, which may also be delayed in perception or in its subsidence, and so referred to the wrong area; this occurs especially during regeneration. In testing for exaggeration of painful impressions the method must be reversed, as an increase of discomfort is more easily appreciated than a diminution.

(3) The Sense of Position of the joints is tested by fixing the limb above the joint to be tested, and then while the patient's eyes are shut, moving the distal part several times and asking him to name the final position. It is well before he closes his eyes to have an agreement with him as to the names to be applied to different positions, as the expressions 'Up' and 'Down', or 'Bent' and 'Straight' seem capable of 
many interpretations. Head has recommended that a chart be kept of the replies, correct and incorrect, for each position, as even a normal individual may make a few errors. In practice, however, it is soon evident whether there is an impairment of the joint sense. This is the better appreciated if the distal joints are tested before the more central ones, for then the improvement in the replies as the distribution of a normal nerve is reached is at once apparent.

6. Trophic Disturbances are finally noted.

Vasomotor phenomena should first be looked for, as they may exist apart from nerve injury, where the main vessel to the limb has been interfered with, or even in purely functional cases, largely in association with disuse.

(a) The pulse should be felt for. Its absence may merely be an abnormal anatomical arrangement of vessels.

(b) Cyanosis of the whole limb is common in functional cases; whereas it is frequently found limited to the ends of the digits in cases of median or posterior tibial injury, especially where there is a lesion of the main vessel also.

(c) Redness limited to the terminal phalanges of the digits supplied by a particular nerve, is frequent in severe nerve injuries and is very characteristic, being most marked in the digit which is solely supplied by that nerve (e.g. little finger for the ulnar, and index for the median) and gradually diminishing as the territory of the next nerve is reached, so that an injury of the particular nerve could be diagnosed at a glance, though the extent of the lesion must be estimated from other factors.

Changes in the Skin and its appendages have next to be looked for.

In association with the redness noted above there is often atrophy of the skin, which is thin, shiny, hairless.

In another type of case the skin is horny and there is a little heap of epithelium under the nail.

Sweating is apt to be excessive in cases of nerve irritation, especially median and posterior tibial cases; while after complete division of the nerve it is absent. The sharp line between the normal skin in one nerve territory and the dry area, or part covered with beads of sweat, is very remarkable; indeed the extent of the anæsthesia can often be forctold from it.

Skin eruptions, especially small papules like a 'sweat-rash', are not uncommon.

Blisters are apt to occur, and when they break to leave chronic ulcers, which are very slow to heal. Their origin can nearly always be traced to trauma, or to immersion of the limb in rather hot water, there being no warning discomfort owing to the anæesthesia; sometimes water which is easily borne by the healthy limb causes blisters on the injured one. 
The Nails show changes in nearly all cases of nerve injury ; these vary from a longitudinal cracking, which is only noticeable from a comparison with the sound limb, to the formation of crooked horns with numerous transverse cracks. Even in mild cases the nails tend to be curved over the ends of the fingers from atrophy of the finger pulp. In cases of arterial injury, the whole nail may undergo dry gangrene, as may the terminal phalanx itself.

The Muscles undergo marked trophic changes in the form of :

(I) Atrophy, which may come on rapidly; indeed the absence of atrophy in a lesion more than a few weeks old, suggests that the paralysis is chiefly functional.

(2) Induration, ending in fibrosis, a condition which is most apt to occur where a vascular injury is associated with a nerve one; though it also develops late in an unrecovered paralysis.

(3) Contractures, which are of two types, the spasmodic, which yields under anæsthesia and is associated with pain from adhesion of the muscle to the nerve, in such a position that its movements pull on the central end of the nerve, or other irritative condition in the nerve; and secondly, the fibrotic, in which actual shortening has occurred in the muscle of a permanent kind. The first type yields almost miraculously to nervefreeing; the second requires tendon lengthening.

The bones and soft parts atrophy also after severe nerve injuries, so that wasting of the pulp and shrinking of the whole ends of the fingers is common, and its distribution in the territory of a particular nerve is at once suggestive of an injury to that nerve, even though the patient may complain of no weakness or numbness.

The joints also usually suffer in association with the other tissues. Apart from limitation due to scar tissue, or contractures of cut or paralysed muscles, they become stiff from a form of dry arthritis, in which the cartilage is eroded, the ligaments become inelastic and shortened in whatever position the paralysed muscles allow them to remain. These changes are not prevented nor cured by daily massage, but after suture of a nerve, as regeneration occurs, the joints tend slowly to return to normal, even before voluntary power is regained, showing that the condition is dependent more on the trophic function of the nerve than on regular movement. As soon as active movements return, the joint improvement becomes rapidly greater.

These joint changes are apt to be most marked in the knuckles, where injudicious splinting in extension readily exaggerates them. Still, they can be seen developing in a patient who wears no splint at all after the injury and who is on daily massage.

All or any of the above-mentioned trophic changes are evidence of an organic lesion of a nerve, and therefore, are of great value, where a func- 
tional paralysis is suspected. It must, however, be remembered that they do not exclude the existence of a functional element superadded to an organic one.

\section{ELECTRICAL TESTS}

To test with accuracy the electrical reactions of a difficult nerve case requires a special study of the subject and considerable experience, so that it hardly comes within the scope of the general surgeon, and those who are interested in the subject should consult the special works where it is dealt with at length. For practical purposes two phenomena are to be considered in any given case :

(I) The absence of a Faradic response in the muscles. This represents loss of excitability of the nerve to the muscle, which can normally be best stimulated by placing one electrode on the 'motor-point ' of the muscle, i.e. over the point of entry of the main part of the nerve into it ; the second electrode is placed either on the nerve trunk higher up, or on some ' indifferent point ', such as the back. With the induction coil, in a normal case a brisk contraction of the whole muscle is obtained, tetanic in character, i.e. lasting as long as the current is continued. This response is lost when there is a severe injury to the nerve, even in cases where the continuity of the fibres persists.

If no response is obtained by stimulation of the motor-point, the nerve should be tested well above the site of injury, because sometimes its excitability is lost below the lesion, although its conductivity remains so that it can be stimulated in the upper healthy part. When the main nerve is thus stimulated, all the muscles which it supplies respond, whereas stimulation of the motor-points enables individual muscles to be tested separately.

(2) Alteration of the response to Galvanism is the other important guide to the condition, and indeed provides more information than the mere loss of faradic response, which may represent merely a temporary block in conductivity. Normally the galvanic current stimulates a muscle to contract through its nerve-supply, but after the nerve has been destroyed, a response is still obtained by direct stimulation of the musclefibres. The galvanic current gives no contraction while it is flowing through the muscle, but only at make and break, best at make with the kathode as active electrode (KCC). No tetanus is obtained as with the faradic, which really represents a series of very rapid makes and negligible breaks; the galvanic contraction is a single sharp one which rapidly spreads from one end of the muscle to the other, beginning at the motorpoint. When the nerve has been destroyed, the contraction is totally different in character, taking the form of a sluggish wave which passes slowly down the muscle as the current stimulates each muscle fibre 
successively, instead of simultaneously through the nerve. The contraction may start at the anode (ACC). Relaxation is as slow as contraction or more so. The muscle may respond to a weaker current than the intact nerve, i.e. the irritability may appear to be increased. This slow contraction is of great diagnostic value in pointing to a loss of function of the nerve. After nerve-suture, an increased briskness in the galvanic response may be the first evidence of regeneration.

With incomplete lesions or regenerating nerves, a response to stimulation of a particular muscle may be masked by a rapid contraction of its direct antagonist. This phenomenon is regarded by some observers as diagnostic of recovery of the nerve. It must not be confused with that overflow of the current to all the muscles of the limb, which occurs when a muscle fails to respond to an ordinary strength of current and a very strong one is used.

Sometimes both faradic and galvanic responses are unobtainable, because :

(a) the skin resistance is increased by thickening, trophic changes, or subcutaneous infiltration from chronic sepsis; in such a case massage and baths for a few days may eliminate this hindrance;

(b) the patient may be unable to tolerate even a weak current. This happens in cases of irritation of the nerve, and sometimes at a certain stage of recovery. This can often be overcome by a course of weak galvanic baths.

To sum up, if a serious nerve lesion is diagnosed on clinical grounds, and the faradic response is unobtainable, while the response to galvanism is slow and worm-like, then an exploratory operation is definitely indicated.

\section{SPECIAL DIFFICULTIES IN DIAGNOSIS}

No apology is made for including observations which have been previously noted by other workers, for although nearly all the points can be found recorded somewhere, yet, in the author's experience, they have not obtained general recognition. Indeed, even when one is alert to the numerous possibilities of error, one may still be misled by an abnormality of nerve distribution which could only be diagnosed after an exploratory operation, and this is well illustrated by some caserecords at the end. The practical importance of remembering the possibility of abnormal nerve distribution is that it often justifies early exploration in cases which are not improving, even though the lesion appears to be an incomplete one, because the symptoms may be due to actual division of one nerve, so that spontaneous recovery would never occur.

All the points noted below have been found of practical value in 
actual examination of the numerous cases of peripheral nerve injury which were treated in the Edinburgh War Hospital (Special Military Surgical Section).

The subject will be considered under the following headings: (I) The patient's mentality: (2) Sensory difficulties; (3) Motor difficulties; Abnormal nerve distribution; (5) The interpretation of electrical tests.

I. Patient's Mentality. Naturally the difficulties of the observer are much increased if the patient, owing to mental inertia, or some other reason, refuses to take an intelligent interest in his own case, as all the evidence of nerve function is obtained through the medium of the patient's answers and actions. Some of the patients who are interested in themselves will in their anxiety (consciously or unconsciously) simulate recovery. If, however, the small precautions mentioned below are used, this source of error will be easily eliminated in respect of sensation.

2. Sensory Difficulties. There are fewer traps in the sensory examination than in the motor. It must be remembered that when a limb is cold, sensation is much less acute. Thus, in cases of recovering epicritic sensation, where there are still punctate areas of anæsthesia, if the patient's hand is cold, he may be unable to feel light touch at all or will localize it wrongly, whereas he may have responded accurately on a warm day; and this accounts for the variations from day to day in the sensory charts of a recovering nerve case. For this reason, the man should always soak his hand in warm water for about twenty minutes before the examination. The heat also increases the excitability of weak muscles to electrical and voluntary stimuli, though if too prolonged it weakens their contraction. The patient's eyes must be kept closed while sensation is being tested, as he is apt to confuse the visual stimulus with that of touch, especially if he is anxious for improvement.

In testing Tinel's sign, care must be taken not to be misled by tingling from an adjacent nerve, as in so many regions two or more have an identical course, e.g. the ulnar and internal cutaneous in the upper three-fourths of the arm. By asking the patient to mark out the peripheral distribution of the tingling, this error is avoided.

Muscle tenderness is sometimes hard to tell from nerve tenderness where the nerve runs through the muscles it supplies, as the ulnar does in the palm of the hand.

3. Difficulties in Motor Diagnosis. The motor function of a nerve is the most difficult to test when it is impaired, because the movement characteristic of most muscles can be imitated by the combined action of neighbouring muscles; or if the muscle is too weak to move the joints, and the observer feels its belly for evidence of contraction, he may be misled by the tightening of the fascia over the paralysed muscle, due to the pull of other muscles connected with that. This last difficulty 
arises particularly in reference to the pronator radii teres and flexors of the wrist, which all arise from the walls of the same compartment of the deep fascia of the forearm. Similarly the fascia is drawn tight over the extensor communis by contraction of the carpal extensors, and this closely imitates a contraction of an atrophied extensor communis itself.

When a muscle is weak or paralysed, the brain stimulus, like the electrical one, is apt to overflow to all the neighbouring muscles, to the whole limb, or even to the whole body, and these widespread disturbances mask any small contraction of a weakened muscle. A sensible patient can be taught to eliminate these accessory actions, but in a man of weak intellect or will-power they form a great cause of difficulty in the examination and re-education of recovering muscles.

The following are the more common instances of simulated action which are liable to cause error.

\section{Substitution in the UpPer Limb.}

(a) Abduction of the arm at the shoulder-joint, normally carried out by the deltoid, can be executed by a hypertrophied supraspinatus.

(b) Flexion of the elbow, in the absence of the biceps, can be partially carried out by the supinator longus and pronator radii teres, and if the triceps contracts alternately with these, the biceps may be lifted forward in simulation of a weak contraction.

(c) Extension of the elbow, in cases of paralysis of the triceps, may occur as a rebound from vigorous flexion, especially if gravity is allowed to act; therefore the triceps should always be tested with the arm abducted and the elbow working in a horizontal plane. When one or two heads of the triceps are paralysed while the third is acting, it is often difficult to be sure which is affected, as the deep fascia tightens over the whole muscle.

(d) When both pronators are paralysed, pronation can be obtained for about half the normal extent by means of the supinator longus, and, if the flexor carpi radialis happens to have escaped, even better by that.

In cases of complete division of the median just below the supply of the pronator radii teres, when therefore the pronator quadratus must be paralysed, we have observed that pronation lacks the last few degrees, and also lacks force. The converse lesion is rare, and would be difficult to verify.

(e) When all the three carpal flexors are paralysed, together with the finger flexors, which have a subsidiary action on the wrist, that joint can still be palmar-flexed by the extensor ossis metacarpi pollicis.

A weak contraction of the flexor carpi ulnaris is difficult to confirm, as its tendon is always lifted forward, during flexion, by the flexor carpi radialis, and its belly difficult to isolate. 
With regard to the palmaris longus, the middle-finger tendon of the sublimis is easily mistaken for this muscle; this is prevented by holding the patient's fingers straight and the wrist in only slight flexion; the sublimis tendon stands forward most in the last stage of strong closure of the fingers.

$(f)$ Flexion of the interphalangeal joints of the thumb and fingers is caused, in the absence of active contraction of the flexors, by the increased tension on their tendons which results during full dorsiflexion of the wrist and knuckles. This is much more marked if there is a considerable degree of contracture of the flexors. It is to be noted that

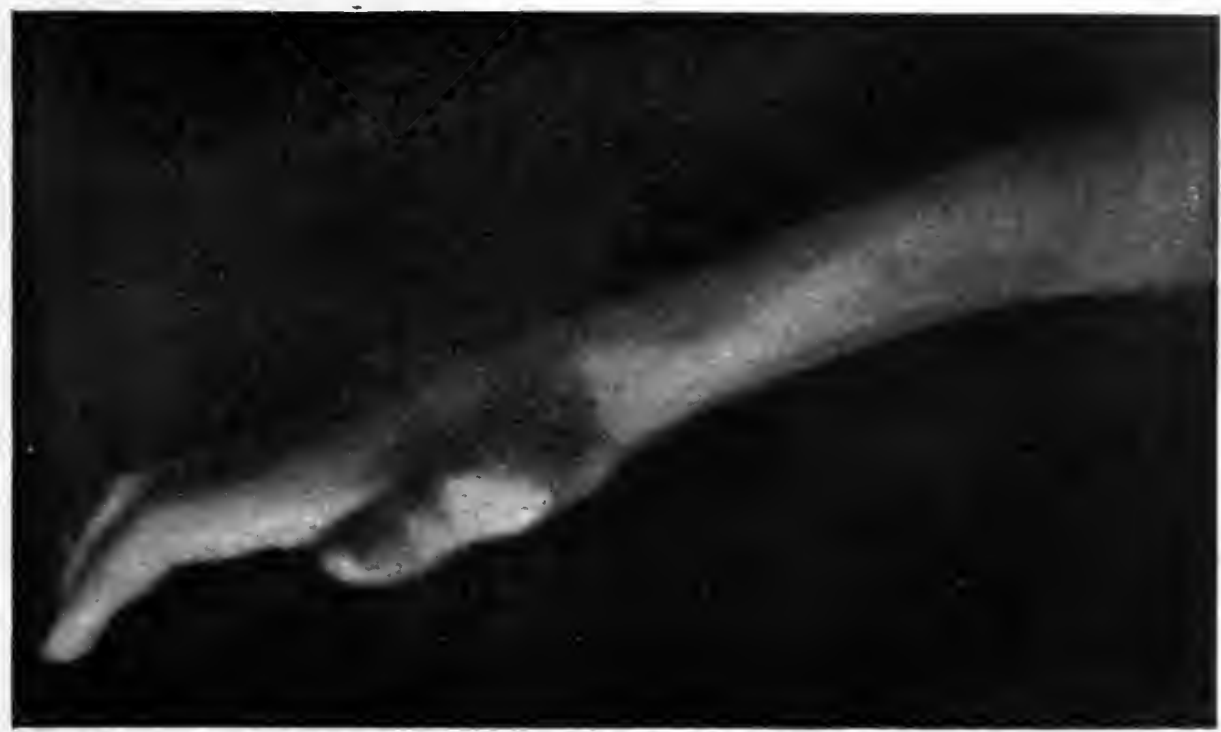

FIG. 9a.-Complete median and ulnar paralysis-hand extended. Action of extensor communis does not quite straighten interphalangeal joints.

when the first weak voluntary movements return in these muscles, they are only able to move the finger-joints in this hyperextended position, as they are incapable of dealing with any slack in their tendons. This corresponds to the well-known persistence of wrist-drop after recovery of the musculo-spiral nerve in some cases, where no cock-up splint has been worn during the paralytic stage to prevent over-stretching of the toneless extensors. The same phenomenon can be noted in the toes. (Figs. $9 a$ and $b$.)

(g) Extension of the wrist can be induced by a similar process through full flexion of the fingers, especially if the wrist extensors are at all shortened by splint treatment.

(h) The return of power in the extensor communis is particularly 
hard to diagnose, as the synergic contraction of the unimpaired flexors of the wrist causes a falling of the wrist, while in that position the interossei alone would be sufficient to extend the fingers. It must not be forgotten that the interphalangeal joints normally depend for extension on the intrinsic muscles of the hand, although, if all the finger flexors are paralysed by a combined median and ulnar lesion, the unopposed extensor communis is then able to extend all the joints; as progressive recovery of the flexors occurs, the action of the communis on the interphalangeal joints diminishes proportionately, until finally it is restricted to the knuckles alone. Then, if no return of power appears in the

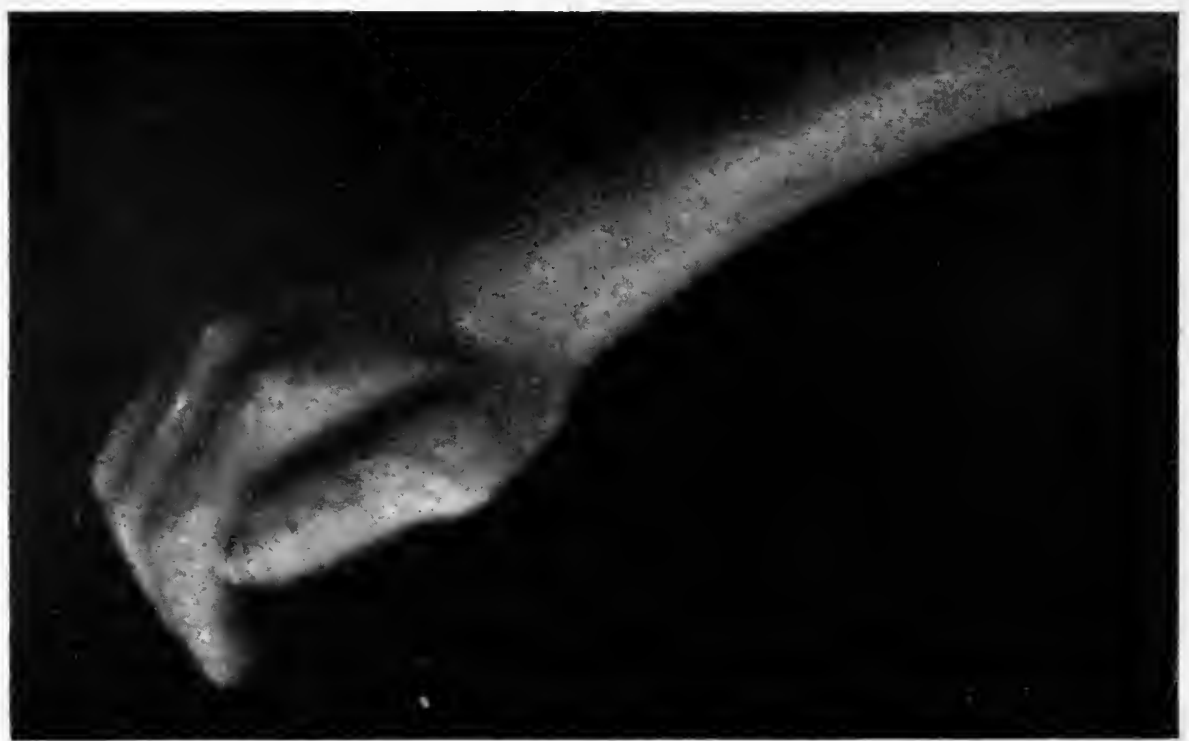

FIG. 9 b.-Same case-trick flexion of fingers by means of dorsiflexors of wrist.

intrinsic muscles, the long flexors roll the finger up on itself, in spite of all the efforts of the extensor communis, which only causes clawing, and the digit is useless for picking up objects.

(i) Extension of the terminal joint of the thumb is caused by the thenar muscles, though not with the same power as by the extensor longus pollicis.

(j) As has been pointed out above, the extensor action of the interossei and lumbricals is not easily substituted, except by the one group for the other. As far as our cases showed, the average extension due to a lumbrical was about equal to that obtained by the interossei which act on the same digit, and conversely; though in certain cases of ulnar paralysis the two fingers with a median supply to their lumbricals showed 
very weak extension, while other cases of median paralysis showed a similar weakness of those digits although all the interossei were acting.

The abductor action of the interossei is readily imitated by the extensor communis, except, of course, that the patient is unable to separate the fingers while keeping the knuckles flexed. The adductor action of the small muscles we have never seen imitated, except perhaps feebly by the extensor indicis; but no patient with true ulnar paralysis could ever show any gripping action by adjacent digits. Tinel reports that all except adduction of the little finger can be done by substitution.

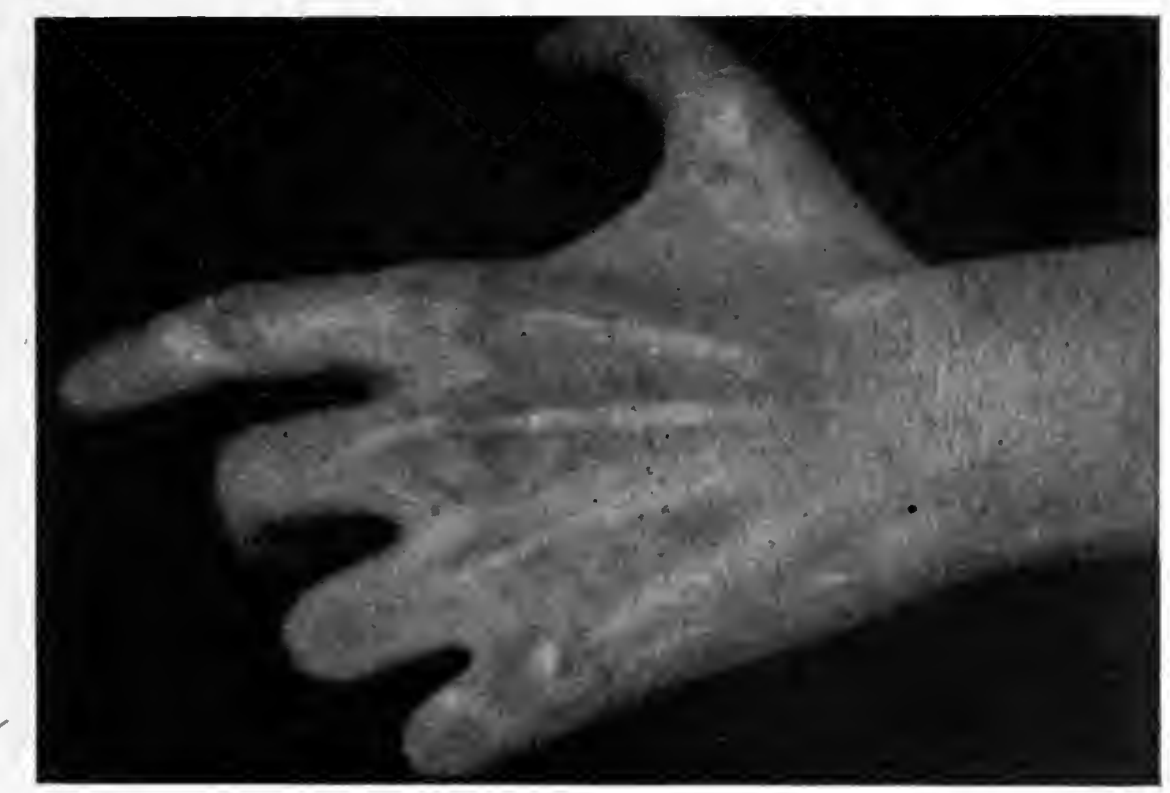

FIG. 10.-Abduction of the fingers carried out by the extensor communis in a case of complete ulnar paralysis.

Of course, cases must be discriminated where the median nerve supplies more than its usual muscles, and vice versa. (Fig. IO.)

(k) Paralysis of the thenar muscles which are supplied by the median nerve (opponens, abductor brevis, and superficial head of flexor brevis) is often difficult to diagnose, although it may be suspected when there is flattening in the region of these muscles. The deep head of the flexor brevis (ulnar supply) is often well developed and can oppose the thumb to the little finger with great vigour, while the extensor ossis metacarpi (abductor longus) abducts it well. Even the combined action of these two, however, fails to give the rotation of the whole thumb which is associated with contraction of the opponens, and which can be appreciated 
by watching the direction that the nail faces. When the opponens and extensor ossis act together, they exactly imitate the action of the abductor brevis.

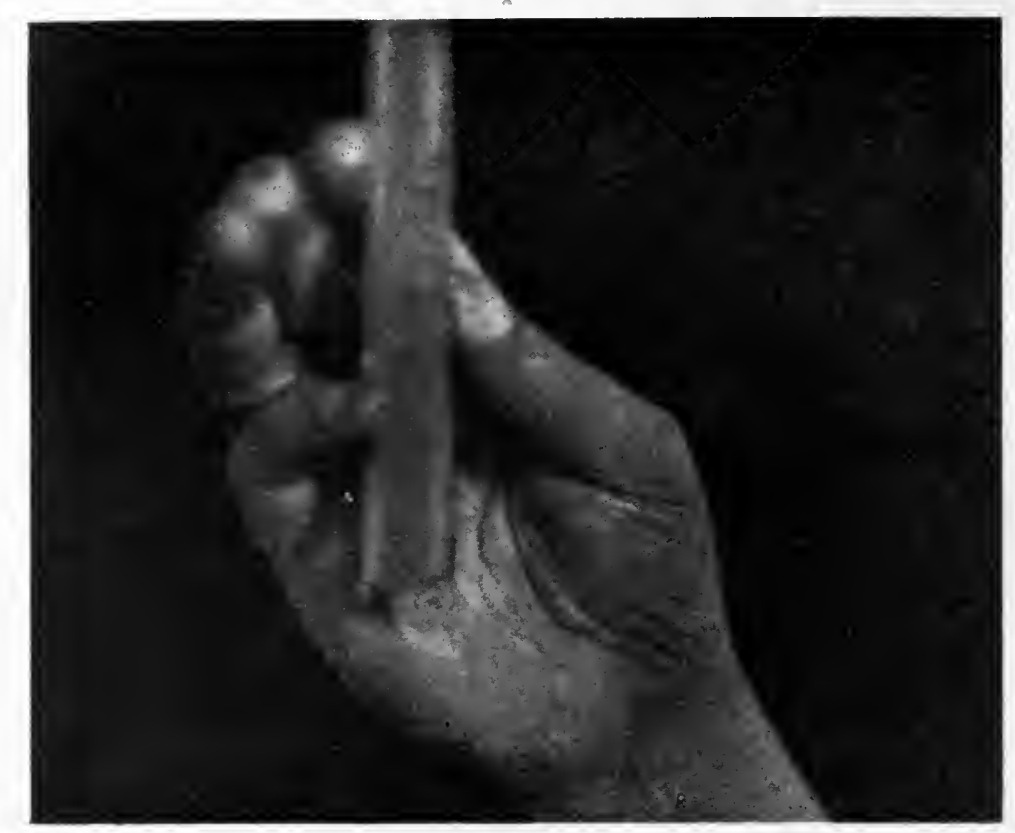

FIG. II.-Pseudo-opposition of thumb by deep head of flexor brevis (ulnar) in case of complete median paralysis.

Substitution in the Lower Limb.

(a) Flexion and extension of the knee, in the absence of active movements, is apt to be produced by a rebound after the contraction of the opponents of the paralysed group, especially if aided by gravity, and therefore these movements should be tested in the neutral position, and preferably in the form of resistance to the observer's hand, rather than as an actual movement; in cases of great weakness, the patient should be asked to support the limb against gravity by the suspected muscles. In this way tone may be detected in the muscles, even if no palpable contraction is obtained.

When the quadriceps is not acting, the tensor fascire femoris is found to have a weak extensor effect on the knee. Similarly the hamstrings are feebly imitated by the gracilis, and of course also by the gastrocnemii, but they are usually involved by the lesion which affects the hamstrings.

(b) Plantar flexion of the foot, in internal popliteal paralysis, can be slightly obtained by the action of the peroneus longus. 
(c) Eversion of the foot, when the peronei are paralysed, can be fairly well carried out by the extensor longus digitorum, which also has a strong dorsiflexing action on the ankle when the tibialis anticus is unable to act.

(d) Inversion of the foot, it must be remembered, is caused by either the tibialis anticus or tibialis posticus, so that if only one of the popliteal nerves is injured, it is not lost ; if both are affected, the other foot muscles are not likely to escape, and indeed none other has this action. Inversion of the heel is dependent on the tibialis posticus, the anticus affecting the part in front of the os calcis chiefly.

(e) Flexion of the toes, like that of the fingers, can be imitated by the claw effect of the long extensors, when the intrinsic muscles are out of action.

- It is necessary to add that patients rarely complain.spontaneously of paralysis even of all the flexors of the toes and of the intrinsic muscles of the foot, as they would do with a corresponding lesion of the upper limb, and they may not notice the anæsthesia of the sole and toes; this explains the low percentage of operations which have been done on the posterior tibial nerve, as small degrees of sensory and motor impairment, which would be considered indications for operation on the nerves of the forearm, are not so regarded in the leg, and often are not noted at all.

The late stages of a posterior tibial paralysis may cause considerable discomfort to the patient, owing to the development of clawing comparable to that which follows ulnar and median paralysis, and also occurrence of trophic sores, resulting from friction of a boot or from warming the foot in front of the fire. In this hospital we have not had the opportunity of examining sufficient calf wounds some. years after infliction to determine the proportion which develop such changes.

4. Abnormal Distribution of Nerves. These cases are impossible to diagnose with certainty before operation, and they are liable to be reported as rapid recoveries, unless they are early and carefully examined after operation.

In the Edinburgh War Hospital several cases have been seen where the median nerve supplied all the intrinsic muscles of the hand, though not the whole ulnar skin area. One case was seen where some of the median muscles were supplied by the ulnar, though again not its skin distribution.

The anatomists, especially Quain, and Poirier and Charpy, quote numerous observations of an anastomosis between the median and ulnar nerves in the arm or forearm, as well as that of the cutaneous branch in the hand. On a few operation cases here, a communicating twig has been observed, though usually such would have been injured by 
the gunshot wound. It was not noticed in the cases which showed its presence clinically, having probably lain well out of the field of operation.

In none of the following cases were the sensory and trophic functions of the injured nerve taken over by its fellow, but they only showed improvement after nerve suture, and at a period when regeneration might well have occurred.

\section{Case r.-Median supplying ulnar muscles.}

Pte. S., rst R.S.F. Gunshot wound of left elbow, Nov, r, r9r6. Partial ulnar paralysis.

Before Operation, at Bangour, May 2, 1917.-Found to have scar adherent to back of humerus, tender, with referred tingling down little finger. Paralysis of hypothenar muscles and $3^{\text {rd }}$ and $4^{\text {th }}$ dorsal interossei, not of other ulnar muscles. Ulnar ancesthesia (not on ring finger at all) to wool, prick, pressure ; not joint sense. Hyperalgesia of the paralysed muscles. Trophic changes slight.

Operation, May 8, by Col. Stiles.-Stiff elbow manipulated; sinus into internal condyle cleaned out. Ulnar nerve found embedded in scar in its course round internal condyle, and to consist of scar, tissue for an inch and a half except for a narrow bundle of fibres along its inner aspect. An inch and a quarter resected; nerve transposed by dividing flexor origins; sutured with elbow at right angle.

Healed in three weeks, a little serum having drained at one point. Motor.Profundus acts, also adductor pollicis, three outer lumbricals, abductor indicis.

After Operatron.-May 19.-Anasthesia unchanged. Trophic.-Hand looks and acts almost normally, the only disability being in little finger. Good grip; no clawing. June 29.-Faradism to ulnar in forearm gave response in : flexor profundus, 3 and 4 ; adductor pollicis ; outer interossei and flexor carpi ulnaris ; the abductor minimi digiti and inner interossei responded to direct faradic stimulation. No response of median muscles during this stimulation, so that the response in ulnar must have been caused by a large anastomosis from median in forearm, not a direct median supply in hand. Sept. 8.- Trophic sore developed on little finger from burn; ulnar tender to wrist. Sept. I6.-Fell on elbow, this lit up the infection; healed October. November.-Contracture developing in little finger, which is cyanosed and shiny. May, r919.-Recovery of all ulnar muscles (except $4^{\text {th }}$ lumbrical), joint and pressure sense (not prick and wool); disappearance of trophic changes formerly noted.

The noticeable features of this case are the retention of power in the radial half of the ulnar intrinsic muscles after resection and suture of the ulnar nerve at the elbow, which can only be explained by the existence of a large communication from the median in the forearm; and the fact that the anæsthesia was complete after the operation, and only became reduced when there had been time for nerve regeneration; the same applying to the trophic changes, which, however, were less widely distributed than in the average ulnar case.

\section{Case 2.-A similar case.}

Pte. McC., H.L.I. Gunshot wound of right elbow, Aug. 8, 1916.

Before Operation.-Nodule on ulnar in wound; 'pins-and-needles' in ring and little finger. 
First Operation, April 17, 1917.-Two and a half inches of ulnar nerve resected at elbow for neuromata in scar tissue. Ulnar transposed beneath flexor origins and sutured with elbow at right angle.

Second Operation, Aug. 21.- Neuroma one inch by a third of an inch found on ulnar in arm, resected; sutured with elbow flexed.

AFter Operations.-Oct. 5--All ulnar muscles act, though small ones weak (note by Capt. Bramwell). Typical ulnar anæsthesia; still some pain in ulnar distribution. Grip good. May, 1919.-Return of all sensation (except to wool of clorsal ulnar branch). All muscles act. Grip, R. 19, L. 24. Writes with left hand.

\section{Case 3.-Similar case, but less extensive median supply.}

Pte. McC., Munster Fus. Gunshot wound of left forearm, Sept. 24, 1916.

BeFore Operation, Jan. 28, 1917.-Paralysis of all ulnar small muscles except adductor pollicis and abductor indicis. Ulnar anæsthesia to prick and wool. No trophic changes.

Operation, Jan. 30.-Transposition and suture of left ulnar, which was found hard for half an inch just above branch to flexor carpi ulnaris, which was spared. Elbow flexed to $60^{\circ}$.

- After Operation.-Feb. 6.-Abductor indicis and adductor pollicis acting; not profundus or flexor carpi ulnaris. April 13.-Recovery of flexor carpi ulnaris. June 15.-Recovery of profundus 3 and 4 (fceble). June 9.-Trophic sore on little finger; contracture of it has developed. Oct. Io.-Still complete ulnar anæsthesia to wool, prick, pressure, joint and muscle pain. Abductor minimi digiti acting feebly.

Case 4.-This case illustrates the converse, i.e. supply by the ulnar of median muscles apparently a rarer phenomenon

Pte. C., W. Riding.-Gunshot wound of left arm, May 20, 1918. Ulnar and median injury.

Before Operation, July 15.-Motor.-All ulnar muscles act, and thenar ones; no other median ones. Median anæsthesia to wool, prick, and pressurc. Median and ulnar acutely tender in wound. Trophic.-Median area scaly and cold; no other trophic changes.

Operation, Oct. 24, by Colonel Stiles.--Suture of median and freeing ulnar in arm. Plaque of scar 4 in. by 2 in. found on neurovascular bundle in arm; good faradic response in ulnar, easily freed; median ended in scar; 2-in. gap; no faradic response.

After Operation.-Nov. 20.-Abductor pollicis and flexor brevis pollicis still act, and all ulnar muscles. Jan. Io, 1919.- Profundus I now acts feebly. May 5.-Recovery of all median muscles; flexion of index weak. Ulnar good. Recovery of pressure and joint sense in median.

5. Electrical Tests. Into the elaborate subject of the difficulties in obtaining satisfactory electrical reactions, their significance, and their order of return, it is not proposed to enter here, because it may be a useful reminder to mention that the electrical responses are difficult to obtain if the limb is cold, and it should therefore always be given a soak in warm water, before they are attempted.

In nearly all the cases which we have observed continuously, weak voluntary contractions have appeared before a response to faradism : but in several pensioners who had received no treatment for months 
after a nerve suture, and then reported, a faradic response was obtained in muscles which appeared to have made no voluntary recovery; however, immediately or shortly after the application of electricity, voluntary contractions were obtained, showing that the patient really lacked re-education. This provides an argument for giving cases prolonged after-treatment under the surgeon who has seen the lesion at operation.

The testing of the exposed nerve at operation by a very weak faradic current gives valuable information as to the condition of the motor fibres, especially where a positive response is obtained; but this does not eliminate the need for judgement by the surgeon in dealing with partial lesions, where it may prove best to sacrifice a few intact motor fibres in order to suture satisfactorily sensory fibres which have been damaged and which are of supreme importance, such as those of the median. On the other hand, a nerve which fails to respond to faradism at operation may yet have the power of recovery when it is relieved from severe compression, as shown in the case recorded below.

Such then are the difficulties which beset us in determining whether: (a) A given lesion is partial and recoverable, or complete; (b) A case has improved since operation, and whether the improvement is due to the operation.

Case 5.-Lesion at first thought incomplete, because opposition of thumb was powerful. Suture of median in forearm.

Pte. B., age 19. Admitted to Bangour, Nov. 10, 1918. Wounded early September, 1918. Immediate tingling in hand, which cramped into flexion. Healed end of October I9I8.

On Admission.-Punctured entry scars on inner side of left wrist (tender) and elbow. Joints free. Motor.-Paralysis of abductor and opponens pollicis, but powerful flexor brevis which imitated opponens. Sensory.-Median anæsthesia (one and a half fingers) to wool and prick. Joint and pressure sense lost in terminal joint and phalanx of index. Median small muscles analgesic. Median tender in lower third of forearm. Trophic.-Unhealed sore from burn on end of index ; wasting of thenar muscles. Electric.-Galvanic response only in abductor and opponens pollicis. $X$-ray. - Shows a small foreign body in lower third of forearm. Lesion diagnosed as partial one.

Expectant treatment tried till Jan. 29, 1919, i.e. massage, galvanism, strapping to keep thumb opposed; but no improvement.

Operation, Jan. 29, by Dr. Forrester-Brown.-Suture of left median in forearm. Median invaded by scar, and adherent to the deep aspect of radial head of sublimis, in which, in a nodule of scar, lay the foreign body. A few fibres ran down the posterior aspect of the nerve; no faradic response. An inch and a half resected till healthy bundles reached. Sutured with wrist in straight line and elbow a little flexed. Primary healing.

Progress.-Feb. I4.-Hot soaks, massage, and galvanism begun. Feb. 21.Sensory: Return of joint sense. No distal tingling. Motor: Still paralysis of abductor pollicis; can oppose thumb-? how. March 2I.-Sensory: Pressure now felt; prick felt on palm; wool felt on parts of index and thumb. Wrist extends well. Began farm work. March 28.-Sensory: Wool not correctly 
localized; prick felt except at tip of index. Motor: Can now abduct thumb. Electric: Faradic response in abductor and opponens. Trophic: No ulcer now, though he touched finger with a cigarette. March 31.-No anæsthesia. Normal thumb movements. Confirmed by Colonel Stiles. July.-Re-enlisted.

Case 6.-Musculo-spiral freed from callus. Recovery, although no response to faradism at operation.

Pte. B., age 34. Admitted to Edinburgh War Hospital, Jan. 22, I919. Gunshot wound of chest, and left fractured humerus, July 29, 1918. Wrist-drop. Healed Feb. 22, 1919.

ON Admission.-Joints.-Shoulder movements a little limited. Motor.Paralysis of musculo-spiral except triceps and twitch in extensor communis. There seems a little tone in radial and thumb extensors. Sensory.-Radial anæsthesia to wool and prick. Slight neuralgia. Tenderness of supinator longus and extensors. Trophic.-Much wasting of extensors. Electrical.-Diagnosis of incomplete lesion of the nerve, probably compression, by Capt. McDonald, because faint faradic response in thumb and finger extensors, which was immediately overcome by their opponents contracting. Slow galvanic response in extensor carpi radialis longior (A.C.C. greatest). No response in supinator longus or extensor carpi radialis brevior.

Operation, Feb. 5, by Dr. Forrester-Brown.-Musculo-spiral nerve freed from callus. Nerve found to have $S$-twist round spur of callus; nerve thick, hard; but no faradic response except in triceps and supinator longus. Spur of bone removed, and raw surface covered with flap of triceps. Nerve freed and incised longitudinally in several places. Long cock-up splint. Primary healing.

Progress.-Massage and galvanism begun. Feb. 24.-Apparently tone in extensors of wrist. Away six weeks at auxiliary hospital, where treatment was continued. April 28.-Can hold wrist dorsiflexed and extend it in mid-supination. Tone in extensors of knuckles. Extensors filling out. May 8.-Thumb and knuckle extensors just act. May 19.- Seen by Colonel Stiles, who found all extensors acting. May 29.-Can keep wrist dorsiflexed while gripping. June 13.-Can dorsiflex wrist a little against gravity when fingers are flexed. July I I.- Wrist still falls to a straight line when knuckles are extended. November.-Good use of all extensors : grip fairly strong. December.-Discharged for light work.

\section{TYPES OF LESIONS FOUND AT OPERATION}

Before we consider the indications for operation and the particular operation to be selected, it is advisable to review the common types of lesion which we are likely to meet in the nerve when it is exposed at the operation.

A. Complete Division. When the surgeon finds the nerve completely divided at the operation, it is immaterial to him whether the severance was caused by the original missile, or by a splinter of bone, or followed the development of a hæmatoma within the sheath. The only importance of these different origins is that in the latter case the gap to be bridged tends to be short. When a peripheral nerve has been divided, the axones from the proximal portion begin to grow downward, but usually some or 
all of them meet with obstruction before they reach the sheath of the distal end and so they are deflected from the correct path and curl in all directions, some upwards again ; they also branch and thus they form a definite tumour of variable size, the true neuroma. In cases where sepsis has been at all prolonged, there is mixed with the nerve elements

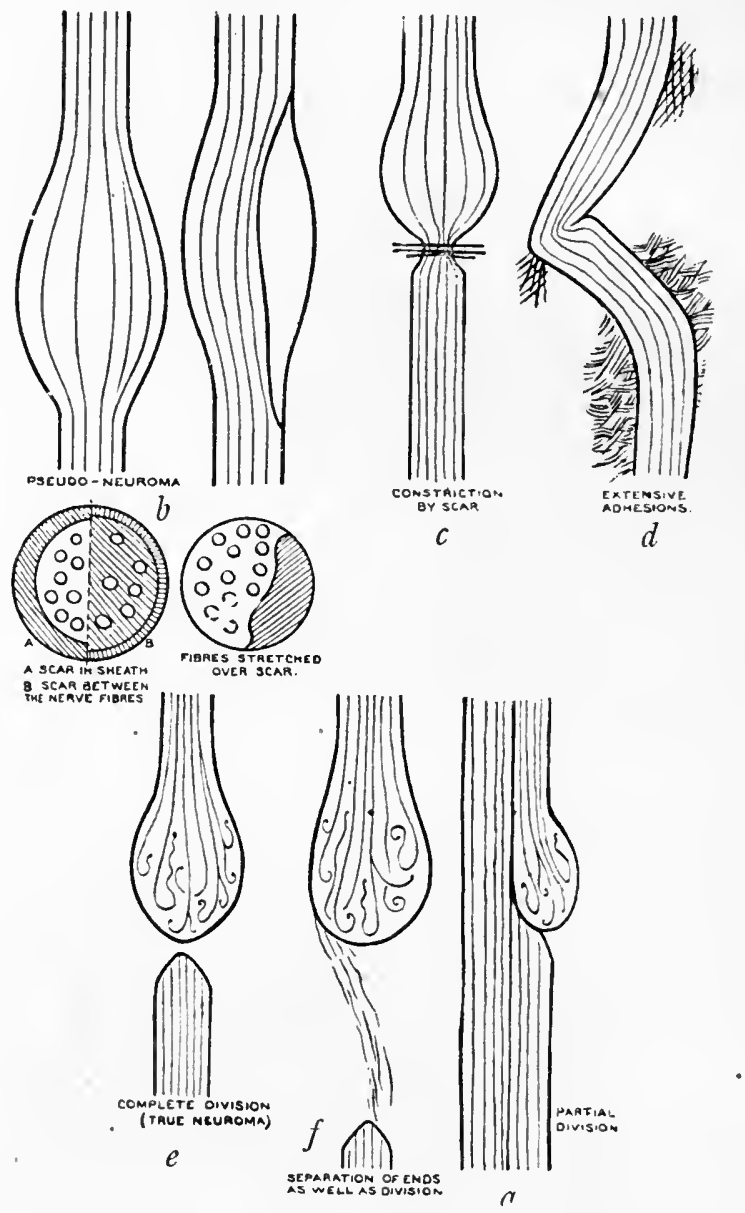

Fig. 12.-Types of nerve lesion.

a considerable amount of scar tissue, which may even preponderate. Under these circumstances the neuroma may attain the size of a walnut ; often it is no larger than a cherry-stone.

The proximal neuroma may be connected to the distal end of the nerve directly, or by a narrow strand of scar tissue, or both ends may be inextricably involved in a mass of scar which infiltrates all the tissues of the part. Whichever of these conditions obtains, it is always difficult to trace the ends at the level of the lesion without inadvertently injuring 
a healthy part of the nerve, and therefore the nerve should always be sought first above and below the lesion where its relationships remain normal. (Fig. I2 $e$ and $f$.)

B. Incomplete Division of the nerve is a not uncommon finding and presents one of the most difficult operative problems, for fibres which have been divided and are involved in even a small amount of scar tissue will not recover unless the ends are freshened and sutured, while to carry this out may necessitate division of other intact fibres. When only part of the nerve has been divided, the affected portion may represent only a few fibres, or a group of fibres which were about to leave the nerve as one branch, or one of the main divisions of the nerve, such as the peroneal portion of the sciatic. One of the heads of the median is not uncommonly affected in this way. Many explanations have been offered to account for the escape of part of the nerve under such circumstances, but none are quite satisfactory. It does appear that, as with large arteries, so with nerves, or parts of nerves, they are more liable to injury in places where they are anchored by the origin of large branches, or by their course round some bone, e. g. the ulnar round the internal condyle of the humerus, and the external popliteal round the neck of the fibula.

In these cases of incomplete division, the proximal ends of the divided axones may form a small neuroma within the sheath of the main nerve, so that when this is found at operation, the destruction of some fibres should at once be suspected. Where the lesion affects a well-defined nerve bundle, it is often easy to strip this up in both directions from the main nerve and suture it, while relaxing the rest of the nerve, but not dividing it also. Where the divided fibres represent more than two-thirds of the whole nerve, the most satisfactory method of suture is to divide the intact fibres and suture all together at the same level. (Fig. I2 a.)

C. The Pseudo-neuroma is another common type of lesion responsible for symptoms of incomplete division. It is consequently sometimes confused with the previous type, which is unfortunate, for the treatment is quite different.

The pseudo-neuroma forms a swelling in the course of a nerve-trunk, similar in feel and perhaps in appearance to the true neuroma, but careful examination reveals the fact that all the fibres of the nerve pass through it without interruption of their continuity; the enlargement is due to the presence of considerable amounts of scar tissue, which may be distributed evenly between the nerve fibres, these latter often being swollen also, or the scar tissue may be in one mass with the fibres stretched over its surface, either as a cord, or a thin sheet. The length of nerve affected by such a lesion varies greatly in different cases, from about $\frac{1}{2}$ in. to 3 in. or even more. (Fig. 12 b.) 
D. Constriction of the Nerve by a localized band of scar may be the only lesion present, though usually it is complicated by the presence of adhesions to other structures or by the development of a neuroma of one of the three types already described situated above the level of the constriction. (Fig. I2 c.)

E. Diffuse Induration of the whole nerve is a common lesion, or possibly of its sheath only, for several inches, or even in the whole length of the limb, as in the so-called ascending neuritis, where the process affects the nerve for considerable distances above the level of the original wound, sometimes even reaching the spinal cord. This induration may be associated with thickening of the nerve, sometimes fusiform, or with a reduction of its calibre for the whole distance. Such a nerve on cross section presents a ground-glass appearance of the cut fibres, and they may be swollen and reduced in number; there is absence of the normal brisk bleeding from the proximal end and the nerve feels hard when palpated; it usually also has a yellowish tinge. Similar changes, in a milder degree, are common in the distal end of a nerve which has remained severed for many months.

F. Adhesions through the medium of scar tissue to other structures are the commonest lesion found after gunshot wounds of the nerves, and they generally complicate all the other types. (Fig. I2 d.)

The exact significance of scar tissue as a cause of nerve symptonis is not yet fully understood. For instance, in some cases where freeing of a nerve from dense scar has been followed by clinical recovery, yet a second exploration done for some other purpose has shown the persistence of a considerable amount of scar round the nerve, often more than in other cases which had been operated on for irritative symptoms and had improved rapidly after the operation. Similarly in cases operated on for tendon injury, one often finds dense scar involving a nerve which has given no evidence of impaired function at a careful clinical examina. tion. This is common with fractures of the ulna, when injury to the inner half of the profundus is being dealt with and the ulnar nerve may be found to run for several inches in a canal of scar tissue, without having given any symptoms.

Where the adhesion fixes the nerve to some tendon in such a position that contractions of the muscle or movements of the joint put tension on the proximal end of the nerve, it is easy to understand the existence of pain and other irritative symptoms. Such a condition is frequently found in cases of so-called functional contracture, which are readily cured by neurolysis followed by suitable splinting. 


\section{INDICATIONS FOR OPERATION}

Where a case of peripheral nerve injury is not steadily improving at the end of three months after healing of the wound, it is a good rule to explore the nerve. The cases which are going to improve spontaneously, almost always do so very soon after the sepsis subsides. Moreover, no case of neurolysis has come under the writers' notice which has been made worse by operation, though a few failed to improve at all. The only cases which lost anything by operation were those in which a small bundle of intact fibres had to be sacrificed in order to suture the main part of the nerve which had been divided. Without operation, such a case would have stood no chance of recovery at all. The large number of cases whose signs suggest an incomplete lesion, while almost complete division of the nerve is found at operation, justify early intervention which saves much time and uncertainty to both patient and staff. Benisti in a recent article recommends the same attitude on the grounds that:

(I) Surgical exploration is without danger.

(2) The diagnosis of a complete division is impossible on clinical grounds alone.

(3) Delay is harmful where complete division exists, and sometimes in other cases also.

The percentage of cases with early nerve symptoms which recovered spontaneously was impossible to determine, owing to war conditions. In numerous cases the presence of signs of nerve involvement was not looked for in the early stages and had cleared up by the time healing occurred. Thus in I9I6-I7, when only healed cases were sent to the orthopœdic wards at Bangour, it was found that in many of them the nerve injury had first been noted, when the question of return to duty occurred after healing of the wounds; in some of these cases at operation a direct wound of the nerve trunk was found, especially in the case of injury to the ulnar and posterior tibial nerves, where in the absence of contracture, the disability is overlooked by the patient till he attempts heavy work. In the early days, few posterior tibial nerves canie for operation, whereas more recently they have formed a fair proportion of the nerve operations. When cases of wounds in the neighbourhood of large nerves and all the fractures were sent direct from France to the orthopædic wards, where a routine examination for nerve injury was made, a large proportion were found to exhibit nerve symptoms at first, though many cleared up spontaneously, or became insignificant as the sepsis subsided. Rarely, if ever, did nerve symptoms first develop during healing, so that a primary wound, rather than the contraction of scar tissue, seems to be responsible for the lesion. 


\section{CHOICE OF TYPE OF OPERATION}

When the nerve has been exposed at operation, the decision as to which procedure will best fit the lesion found, is often one of extreme difficulty, demanding both judgement and experience. It will most often be made wisely, if the preliminary clinical examination has been careful and thorough. In this connexion it may be well to refer to the great variation in interference with function presented by different nerves.

Thus with the musculo-spiral, sensory loss is negligible, provided the muscles which it supplies are working well; whereas there are no others which can substitute their function efficiently. On the other hand, its motor function is coarse, compared with that of the intrinsic muscles of the hand, which accounts for the relative rapidity of its return after nerve-suture; probably also the so-called 'complete' recoveries of the musculo-spiral have a persistence of as much anæsthesia, as would disable the patient seriously, if it were in the median distribution.

Median anæsthesia, on the contrary, is most disabling, even in mild degrees. For instance if a man with full use of all his forearm and hand muscles, but with anæsthesia of the tips of his index finger and thumb (a common clinical picture), be asked to button his coat, he will fumble about and be unable to perform this simple act, because he lacks the sensory information, which should guide the movements. On the other hand the motor function of the median can be largely substituted by the ulnar. The practical bearing of this is that every effort should be made to restore sensation in median injuries, even if this entails the sacrifice of motor branches, especially as these can largely be compensated for by tendon-transplantations from the extensors.

In the case of the ulnar, as with the musculo-spiral, it is the motor function which is predominant in importance; but it is the lowest, hand, branches, not those in the forearm, which matter most. Loss of the ulnar intrinsic muscles gives in most instances, though not in all, a very weak hand. Its anæsthesia is more troublesome than that of the musculo-spiral, because it involves the ulnar border of the hand and the little finger, which are apt to lean on rough or hot objects such as radiators, and so acquire traumatic ulcers, which are slow to heal

Combined partial lesions of the median and ulnar with loss of all the intrinsic muscles of the hand are most disabling, as the long flexors curl the fingers up, when any attempt is made to grasp an object, owing to the loss of the steadying extension of the interossei and lumbricals on the interphalangeal joints and their flexing power on the knuckles. If either a lumbrical or interosseus remains acting on a finger, this effect is scarcely noticeable; it is the loss of both which is so serious 
Paralysis of the long flexors eliminates the deformity, but leaves the digit useless. The disability can to a certain extent be overcome by a tendon-transplantation.

Loss of the thenar muscles can be partly compensated for by an arthrodesis of the first carpo-metacarpal joint so that the first and second metacarpals form an angle of $60^{\circ}$, which is the common functioning position of the thumb; but though this gives the delicate pincer action, it does not permit of the strong adduction of the thumb on which its powerful grip depends.

Complete sciatic paralysis has been noted in some cases to give very little disability, apart from the liability to trophic ulcers. Paralysis of the external popliteal alone, on the contrary, is apt to lead to deformities from the unopposed action of the flexors, while its sensory and trophic disturbances are insignificant. Injury to the internal popliteal is likely to be followed sooner or later by troublesome trophic sores and clawing of the toes, both of which are most disabling for walking; incomplete lesions also frequently have severe neuralgic symptoms.

The disability due to the nerve injury having been duly weighed, it remains to consider what procedures for remedying it are at our disposal.

To deal first with complete lesions, we may refer shortly to the possibilities of overcoming a given gap in various nerves at different levels.

The routine which we would advise is as follows:

I. Extensive freeing of the nerve. This alone gives $I$ in. to any nerve in the whole length of the arm, or forearm, and $2 \mathrm{in}$. in the thigh or leg. This depends on the inherent clasticity of the nerve.

2. Relaxation of the nerve by temporary fixation of joints in suitable positions.

Adduction of the arm at the shoulder gives an additional I in. after freeing to all the nerves which cross the axilla, i.e. musculo-cutaneous, musculo-spiral, median, and ulnar.

Flexion of the elbow after freeing gives 2 in. to the nerves which pass in front of it; only $I$ in. to the musculo-spiral which passes from front of elbow to back of forearm.

Extension of the elbow gives I in. to the ulnar which passes behind it.

Flexion of wrist gives about I in. to median and ulnar.

Flexion of the knee gives 2 in. to the sciatic and its branches.

Plantar flexion of the ankle gives $I$ in. to the posterior tibial and its branches.

This device is not found to permanently limit the joint, as might have been feared. If a six weeks' interval is allowed to elapse after the 


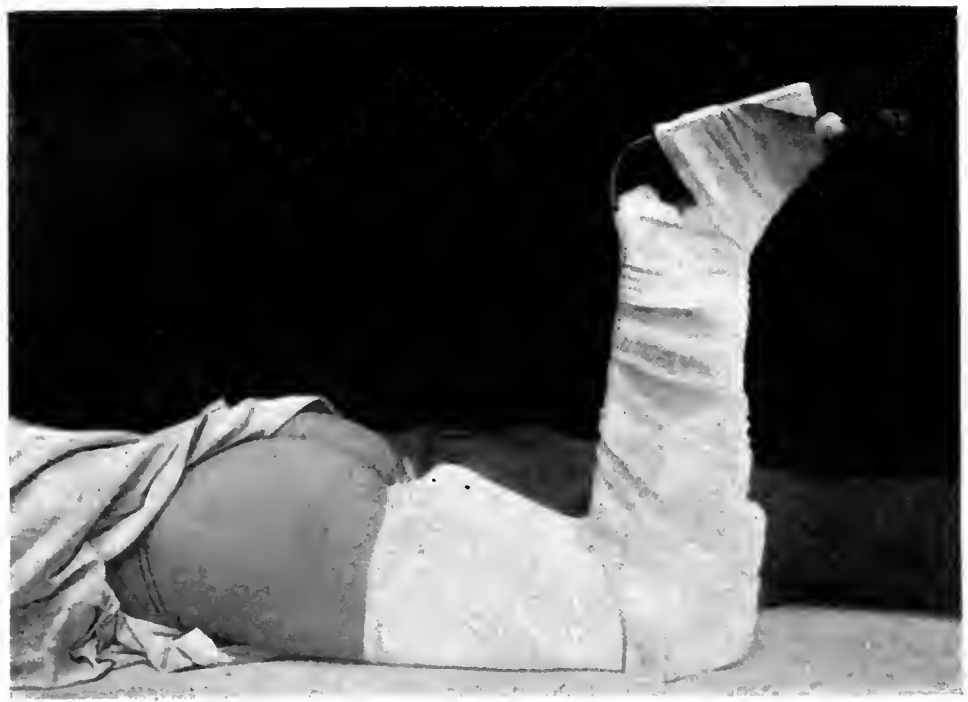

FIG. I3.- Splinting after suture of posterior tibial nerve (or sciatic). Note flexion of knee and plantar-flexion of ankle.

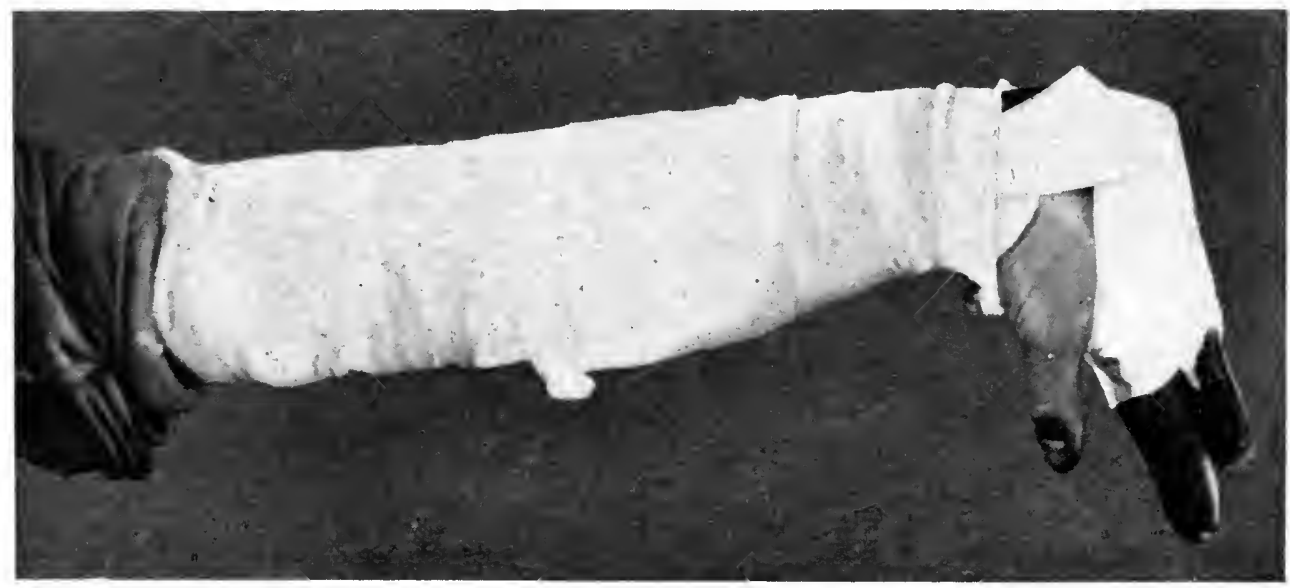

Fig. I4.-Splinting of limb after suture of ulnar nerve, when it has not been transposed. Note extension of elbow, with flexion of wrist to right angle.

operation before any stretching is allowed, and the force used is strictly controlled by the guidance of pain, it will be found that the range of joint increases rapidly without danger of separation of the nerve-ends, nor irritation of the nerve. Some of the cases with extreme tension have recovered the nerve function early.

3. Alteration of the course of the nerve. The transposition of the 
ulnar from the back to the front of the elbow-joint is the most striking instance of the value of this procedure, as it is then relaxed $2 \mathrm{in.}-3 \mathrm{in}$. by flexion of the joint instead of the $I$ in. previously gained by extension. The musculo-spiral sometimes benefits by being brought to the front of the humerus from the back, but this depends largely on the line of the humerus after a fracture; forward bowing of the bone makes the anterior route longer.

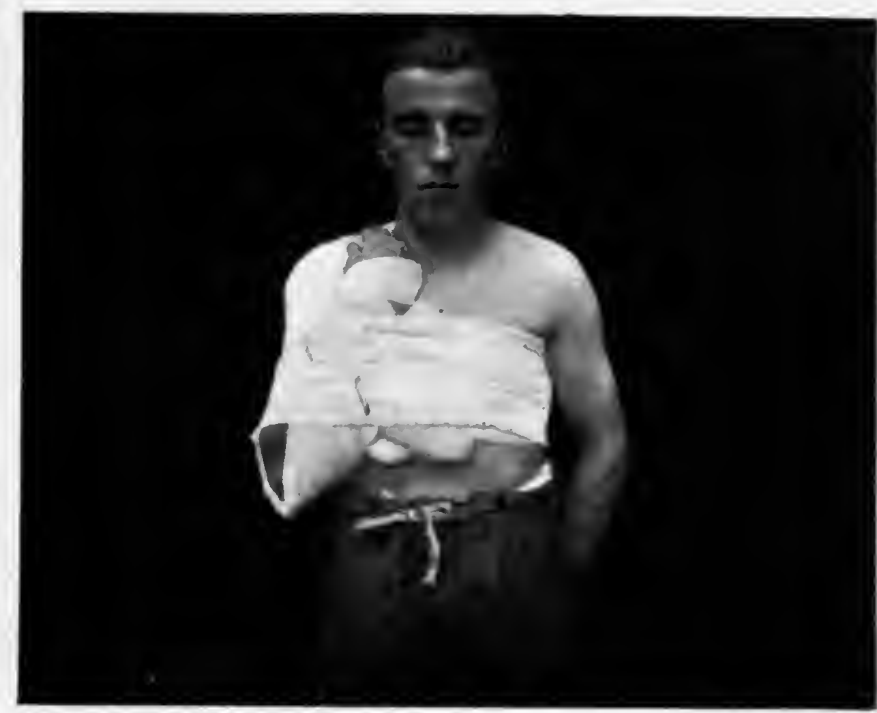

FIG. 15-Bandaging of limb after suture of median and ulnar (if transposed). Note flexion of elbow, adduction of arm, and slight flexion of wrist

Other nerves whose course may sometimes with advantage be altered are the median by bringing it superficial to the pronator teres, and the posterior tibial superficial to the soleus, so as to gain the full benefit of flexion of the corresponding joints, instead of letting the nerve be held at an angle by the muscle.

4. Stripping up of branches which anchor the nerve and prevent concentration of its slack at the required point after freeing. As noted in the anatomical section, certain groups of branches lend themselves to expensive stripping up from the main nerve, and in our experience we have not found that their function suffers by this procedure. It is only where the lesion is distal to these branches that freeing them relaxes the nerve much, allowing its proximal end to be drawn down till the original point of origin of the branches lies distal to their entry into the muscle, ..e. relaxing them. Where the lesion is above their origin, they themselves become tense almost at once. (Fig. I6.) 
5. Sacrifice of branches. It must always be remembered, when suture is prevented by tension of branches distal to the lesion, that these are already functionless, and therefore it is better to suture the rest of the nerve at their expense, than none of it. Many motor branches can be compensated for by an immediate or later tendon-transplantation.

6. Shortening the bones often enables a gap to be overcome, which can be so in no other way. If an ununited fracture is already present, e. g. humerus or radius, this is frequently the best treatment for the bone as well as the nerve condition. Where, however, the bone is intact, it must be remembered in connexion with war injuries that there is the ever-present possibility of a flare-up of sepsis, and if this involves a bone, non-union may result, and convert what was a disability into a disaster.

7. The two-stage operation must be the resort when all other

a.

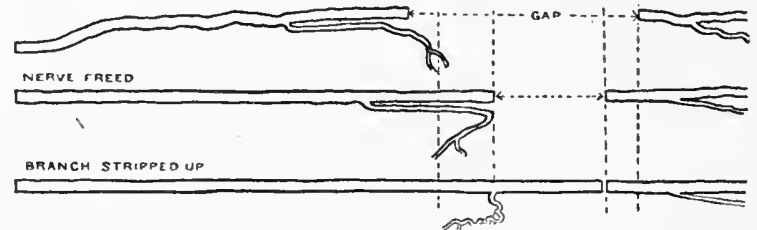

FIG. I6.-Diagram to show effect $(a)$ of freeing nerve; $(b)$ of stripping of branches and letting them take a recurrent course in overcoming gap in a nerve.

methods of direct suture fail. The principle is to bring the untrimmed ends of the nerve into contact, .or as near as possible by the methods of relaxation described above, and then anchor them by strong sutures. Within 2-3 weeks stretching of the nerve is begun through the medium of the joints and is carried out as rapidly as pain will allow, so as to forestall any anchoring of the nerve by adhesions; as soon as the full range is obtained, the nerve is explored again and by repeating the process enough slack gained to allow of resection of the damaged ends. The gain at the second operation is not nearly as great as the first, for the nerve does not readily regain elasticity. This method is applicable to a nerve unavoidably explored in the presence of sepsis, with the modification that no extensive freeing is done, to avoid opening up healthy tissues to the infection. (Fig. I9, p. 79.)

By the above technique the following gaps have been dealt with :

Median, $4 \frac{1}{2}$ in. in the arm of forearm.

$6 \frac{1}{2}$ in. in forearm if motor branches sacrificed in forearm.

Ulnar, $3 \frac{1}{2}$ in. in arm or forearm ; 5 in. if transposed to front of elbow. 
Musculo-spiral, 3 in. ; $3 \frac{1}{2}$ in. or 4 in. if transposed to front of humerus.

Sciatic, 4 in.

Posterior tibial in upper one-third of leg 4 in. below its main branches 6 in.

It must be remembered that with more extensive destruction of nerve than is represented by the above figures, there is sure to be such widespread injury to the other tissues, as to render the limb of little value, even if regeneration of the nerve were obtained, which makes its destruction of minor consideration.

The available figures for nerve-grafting and all the suggested forms of artificial bridge, do not support the value of such. If all other methods fail there can be no harm in trying the introduction of a graft from some large cutaneous nerve.

Tendon-transplantation will be dealt with in a separate section.

\section{METHODS OF DEALING WITH PARTIAL LESIONS}

I. Where two-thirds or more of the calibre of a nerve has been destroyed, and the remaining third does not constitute one of the leashes which can be stripped up for some distance, then it is usually justifiable to sacrifice it in order to do a neat suture of the whole nerve.

2. Incision of the nerve-sheath in several places in a longitudinal direction has been found a useful procedure in cases where there is either great thickening limited to the sheath, or else infiltration of scar tissue among the nerve bundles. The exact rationale of the method is difficult to explain; probably it relieves tension. When this method has failed and serious loss of function persists, then it is usually best to operate again and resect the pseudo-neuroma; but when cases are found not to have loss of continuity of the axones, the conservative method should be given a trial, because there is no certain method of judging which among such cases will recover well and which will fail to improve; even the results of testing the exposed nerve with a faradic battery are not conclusive; for instance, the absence of response in certain muscles does not prove that they will not recover

3. Division of constricting bands of scar and the removal of as much of the scar tissue as possible are methods which should always be applied, though here again the outcome cannot be foretold with certainty. If scar tissue is left in the bed into which the nerve falls back, adhesions are sure to re-form, but the symptoms will not necessarily recur. Where all the scar tissue cannot be removed, part of a healthy muscle or flap of fat should be interposed between it and the nerve. Wrapping round 
with any tissue, even living ones, creates a great danger of strangulation, as shown at second exploratory operations, and therefore cannot be recommended.

\section{PROGNOSIS}

The conditions which affect the prognosis after nerve operations are many of them out of the surgeon's control and their relative importance is difficult to estimate. Tables which were worked out on a series of 500 cases at the Edinburgh War Hospital were inconclusive, except in a negative sense by showing that factors which seemed most unfavourable did not necessarily prevent a good end-result, while cases where all desirable conditions were present, might yet make slow progress, or none. The following factors are those which are likely to influence the result most.

I. The length of time which elapses between the original injury and the operation. In the writer's experience cases have recovered, where the nerve had been degenerated for $2 \frac{3}{4}$ years before suture, and recovery after even longer periods has been described by other observers. On the other hand, a case in which primary healing of a bullet wound occurred, so that the ulnar nerve was able to be sutured at the wrist on the tenth day, showed very slight evidence of recovery after Io months, whereas one might have expected it to occur rapidly in such a case.

2. Long duration of the initial sepsis might be expected to influence the issue unfavourably, both by delaying operative measures and also by reducing the vitality of the central nerve fibres, on whose activity regeneration depends. In the figures at the writers' disposal, this factor could not be proved to have any constant effect.

3. The lighting up of sepsis after operation did appear to act somewhat unfavourably, though not to the extent which one might reasonably expect ; indeed in several cases where severe flares had occurred, recovery after nerve suture took place both rapidly and well, notably in a brachial plexus, operated on the tenth day after the wound on account of pain, while a small sinus was still present; the posterior cord was sutured and suppuration occurred, yet I 8 months later all the muscles which it supplied were acting, while all the other muscles supplied by the plexus were strong, and the only anæsthesia was over the first knuckle (radial). Naturally sepsis is a factor which one would not deliberately risk, but unfortunately it is apt to intervene in spite of all precautions, and at periods as late as two years after healing of the wound. For this reason, it is not advisable to delay the operation for a long period in the hope of avoiding a 'flare-up', because one merely adds to the patient's invalidism, without ensuring safety at the operation. A more rational method is to test the activity of the infection by a course of 
heavy massage to the scars, as soon as these have healed. If this induces a flare-up, it would be obviously unsafe to do a nerve-suture, and the alternatives then are:

(a) to do a radical excision of all the scar tissue, without attempting to get the ends of the nerve together; if primary healing occurs after this, the suture may be done with impunity;

(b) to repeat the course of heavy massage at intervals until no flare occurs; this form of auto-inoculation is equivalent to a course of vaccine treatment, and the dosage is more easily regulated

4. The nature of the initial lesion naturally affects the prognosis greatly.

(a) Incomplete lesions may represent:

(I) Division of a part of the fibres of the nerve, which cannot therefore be expected to recover without suture; while the latter may be mechanically impossible without sacrificing the other intact fibres.

(2) Constriction of the nerve by scar tissue; these cases often show remarkable recoveries after freeing.

(3) Fibrous infiltration of the nerve between its fibres with thickening of the sheath, forming a pseudo-neuroma. Such may improve considerably after multiple longitudinal incisions have been made in the sheath to relieve the tension; on the other hand, they may give most disappointing results after neurolysis, and indeed it may be preferable to resect the whole affected part of the nerve and wait for the regeneration to follow suture, as this has a chance of becoming more complete.

(4) Adhesion of the nerve to neighbouring structures. When this represents fixation of the nerve to a tendon in such a position that every movement puts a strain on the proximal end, there often results a functional paralysis associated with the patient's attempts to avoid pain, and this will be cured almost miraculously by freeing the nerve at operation. Although the contracture can be overcome passively, and is obviously of a spasmodic type and chiefly voluntarily, yet it is useless to treat it by non-operative measures, as is proved by the history of these cases, which have usually had massage and baths for years before they reach the surgeon, whereas they are cured a few weeks after the operation.

(5) Neuritis, possibly of the ascending type which involves the nerve for many inches above the gunshot wound. In these the nerve may not be much thickened, possibly even 
narrowed, but it feels unduly hard and has a yellowish tinge, instead of the normal pinkish white. Such lesions often cause severe pain of the burning (causalgic) type, very resistant to treatment of any form, and though they may slowly recover spontaneously, they seldom benefit by operative measures directed to the site of injury. Where the pain is not improving and is such as to render the patient's life unbearable, division of the nerve in a healthy part above the lesion and primary suture is not only justifiable but necessary. Provided this is done above the level to which the pathological process has ascended, it will cure the pain; unfortunately the neuritis is apt to pass up into the nerve plexuses, or even into the spinal cord, and these cases will be unrelieved by suture of the peripheral nerve.

From what has been said above regarding the nature of the incomplete nerve lesions, it is not surprising to find that the results of neurolysis operations do not compare as favourably with those of nerve suture as one might at first be inclined to expect.

(b) Complete severance of the nerve naturally calls for suture and the prognosis depends primarily on whether this is able to be attained. The question of the possibilities has been dealt with. It may be remarked here that within the period at which the cases have been able to be re-examined, i. e. one to two years, few show absolutely complete recovery, though as they are continuing to progress, there is no reason for supposing that they may not ultimately attain it. On the other hand, very few show no evidence of regeneration, so that it will be only by examining a large series at the end of four to six years after the operation, that any reliable idea of the final prognosis can be attained.

5. The presence of complications in the form of injuries to other important structures may affect the outlook for the ultinate function of the limb so adversely that the nerve injury itself becomes of minor importance. This is a point which is apt to be overlooked unless a systematic examination of every function is made before operation. At the operation some form of tendon-transplant is often of as much importance as the nerve suture, while a bone or joint operation may be an essential preliminary to the latter. It is necessary, therefore, to plan out the whole line of treatment at the first examination, before starting on any operative measure.

The complications of most serious import are :

(a) Fractures of bones with non-union, or with mal-union in such a position as to impair the function of the muscles. These should be 
corrected before the nerve is dealt with, as the forcible manipulations which they entail might throw undue strain on the nerve-suture.

(b) Joint stiffness, usually following prolonged sepsis in the limb. If it involves the joints across which the injured nerve runs, it may prevent that postural relaxation of the nerve which is usually necessary for suture. This is especially apt to occur in sciatic injuries, where a fracture of the femur has been followed by stiffness of the knee in the extended position, and it often renders suture impossible. All sorts of measures have been suggested for meeting this difficulty, but some of them impair the function of the limb more than the nerve injury itself, and as regeneration within a reasonable time can never be guaranteed, it is, in the lower limb, frequently preferable to resort to amputation. In the upper limb, a prelininary arthroplasty of the elbow is suitable.

(c) Tendon complications, such as division, adhesions, or contracture are very common and very tiresome accompaniments of nerve wounds The treatment which benefits them, i. e. early movement after operation, is often contraindicated for the nerve, and much judgement is required in balancing their respective needs.

(d) Injuries to large vessels not infrequently accompany that to the nerve and adversely affect the prognosis, as usually the circulation in the limb remains permanently below normal, so that it is unduly sensitive to cold, while the muscles are subject to sudden loss of power. There is also a greater liability to trophic ulcers after slight trauma than in uncomplicated nerve cases. In this connexion, it must be remembered that any extensive operation for the nerve may impair the compensatory collateral circulation dangerously; this is likely to happen after ligature of the brachial artery, if an attempt is made to suture the musculospiral and the nerve has to be traced round the groove on the humerus, for here it is impossible not to interfere with the superior profunda vessels, which are the main elements in the collateral circulation. In such a case, the nerve should not be dealt with but a tendon-transplant resorted to.

6. The nature of the operation done, naturally has a most important bearing on the prognosis. If it is inappropriate to the lesion, no improvement can be looked for; thus if a nerve is merely freed, while a large proportion of its fibres remains divided, complete recovery must not be expected.

(a) Extensive interference by measures such as transposition of the ulnar nerve to the front of the forearm may influence the outlook unfavourably, but it must be remembered that such is only resorted to when the ends of the nerve are so widely separated that no recovery at all could take place without it. Out of nineteen cases of transposed ulnars, six developed neuromata at the site of suture and had to be re-sutured, 
after which the ones that were observed began to show signs of regeneration. This is a remarkable proportion, as only two were found in all the other nerve sutures. On the other hand, the remaining transposed ulnars showed as good a proportion and degree of recovery as the other nerves.

(b) The degree of tension after suture did not appear to have any constant effect on the result, though some observers have said that the greater the tension the more likely is regeneration to be satisfactory. This has been explained on the assumption that where tension is not great, parts of the nerve sheath are apt to be inverted by the sutures and to form an obstruction to the down-growing axones.

(c) The suture material used will have an unfavourable influence if it is of a kind which induces tissue reaction. The experiments of Sargent have shown that catgut and any absorbable material cause most reaction, while linen-thread and fine artificial silkworm-gut cause least ; I6o linenthread was entirely used in the cases in the Edinburgh War Hospital.

(d) The bed provided for the nerve at operation is important, as the formation of fresh adhesions may induce a recurrence of the symptoms, or prevent regeneration after suture. In the writers' experience, all the artificial means which have been recommended to prevent adhesion, such as Cargile membrane, fat and fascial grafts, not only failed to do so, but were apt to strangle the nerve; whereas exploration of nerves which had been expensively freed at some previous operation for suture, showed only the lightest adhesions where they lay among the normal muscles, though they might be densely adherent at any spot where it had been impossible to excise all the old scar tissue. It seems therefore best, when a complete resection of the scar cannot be done, to interpose normal muscle or fat between the scar and the nerve, but to avoid any wrapping round of the latter, for fear of strangulation.

(e) Finally, as to the outlook when end-to-end suture is impossible: in the series of cases at our disposal, out of $45^{6}$ this occurred $3 \mathrm{I}$ times (6.6 per cent.), mostly in the early cases, when the technique had not been fully evolved. Out of eleven nerve-graft cases, five showed evidence of regeneration and two considerable recovery of function; so that, if it is quite certain that no other method is available, this should be tried, but it certainly does not offer much hope, and the patient should arrange his life as if there were none, otherwise he may become a useless invalid.

The function of the musculo-spiral nerve can be satisfactorily substituted by tendon-transplantation, but the intrinsic muscles of the hand have not yet been so well replaced; and the median anæsthesia is a most serious disability from the point of view of function.

7. After-treatment has great influence on the ultimate result after bone and tendon operations, but as far as one can judge, little or none 
on nerve regeneration. Cases have completely recovered without any massage or electrical treatment, while others on whom every care was expended, have shown but the slowest regeneration. The question of relaxation of paralysed muscles will be dealt with (p. 64), but it must be granted that even the musculo-spiral nerve has recovered its function completely in a few cases, where no splinting was done to counteract the ill effects of gravity. The great value of after-treatment is to overcome the complications, such as tendon adhesions and stiff joints, and by electricity re-educate the patient in the use of muscles, which have so long been paralysed that the brain has ceased to take heed of them, so that return of nerve control necessarily implies return of voluntary power. The most astonishing restoration of function can often be produced late after a nerve suture in a patient, who has not realized that the nerve has regenerated, by demonstrating this to him with a faradic current, and then inducing him to repeat the movements voluntarily.

\section{ORDER OF RECOVERY}

The following table shows the order of recovery of the various functions of the nerves in a series of cases on which we were able to make regular observations for considerable periods. War conditions, with the continual transfer of cases from Base to Auxiliary and back, and the rapid changes of Medical Officers, have made the available records of this nature scanty and incomplete in proportion to the mass of material which was available, a most regrettable fact from the scientist's point of view.

It will be seen from the data given below that a considerable number of cases show definite evidence of nerve-regeneration within eight weeks after suture.

It will be noted that frequently Tinel's sign of tingling on percussion on the nerve at the level which the down-growing axones have reached was the first sign and a valuable one. But we must mention that in two cases which reached almost complete recovery, this sign was absent until long after return of voluntary power in the muscles, although it was carefully looked for week after week.

We have seen no case in which the signs of regeneration did not correspond to a down-growth of axones from above, i. e. where muscular branches came off distal to the lesion and nearer to it than sensory ones, as in the median at the elbow, it was motor recovery which preceded sensory. Where both sets came off at the same level, the order of recovery varied in different cases. In the case of the median and ulnar at wrist, some sensory improvement was noted before motor recovery, 
but this might be accounted for by the more delicate tests we possess for sensory function. If merely a few fibres of the intrinsic muscles had recovered, it would be impossible to detect their contraction.

The larger muscles showed a return of voluntary power before any response to faradism, but the converse was often true of the intrinsic hand muscles, especially in patients who had been discharged early after suture and had no treatment. This probably is accounted for by the smaller degree of interest that patients take in the recovery of these small and complex muscles, whose actions are so well imitated by combinations of other muscles.

Although the Rate of Regeneration after nerve suture has varied considerably in our cases, yet the most rapid recovery of lesions in the upper arm has never equalled that for ones at the wrist, where a shorter distal segment has to be regenerated.

All the evidence available from the cases which have been examined systematically for considerable periods, supports the theory of a downgrowth of new neurons from the upper segment of nerve centrifugally along the distal segment. For example, the order of return of voluntary power in different groups of muscles has corresponded to the level of origin of their branches from the main nerve, or at any rate to the total length of their branches. Where motor branches arise higher than sensory ones, it has been the motor function which has returned first.

No reliable case has been found either of immediate return of function after suture, nor of recovery too rapid to be accounted for by the above theory. Numerous cases have been noted with abnormal sensory and motor distribution of nerves, and these, unless careful records are kept immediately before and after operation, may be mistaken for rapid recovery. The variations in rate seemed independent of the nerve involved, and of all the factors which it occurred to us to study.

Of the cases which have been followed to recovery, nearly all showed some evidence of its commencement by the sixth week after operation, the earliest signs being usually tingling from percussion on the nerve below the line of suture, tenderness of the muscles supplied immediately below the lesion, and the return of deep sensation. If the level of tingling descended steadily week by week, the case usually showed return of other functions at a corresponding rate. The absence of this sign of Tinel's was, however, no proof that recovery was not occurring.

When deep sensation and joint sense had returned, there was often a delay of many months before wool and prick were felt, and even when this was returning, the most distal part of the nerve distribution was apt to remain anæsthetic. 


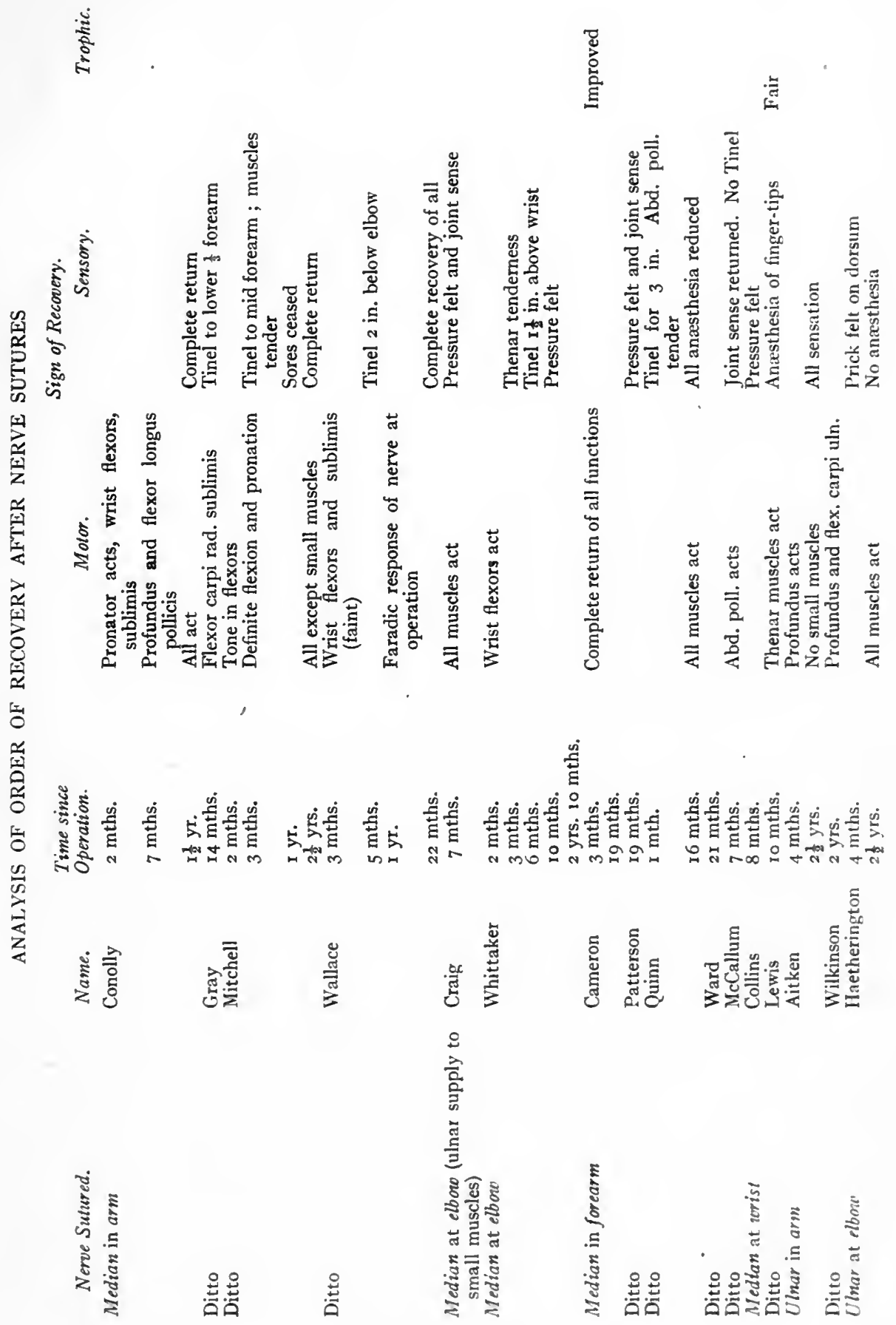




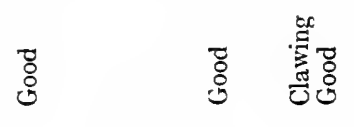

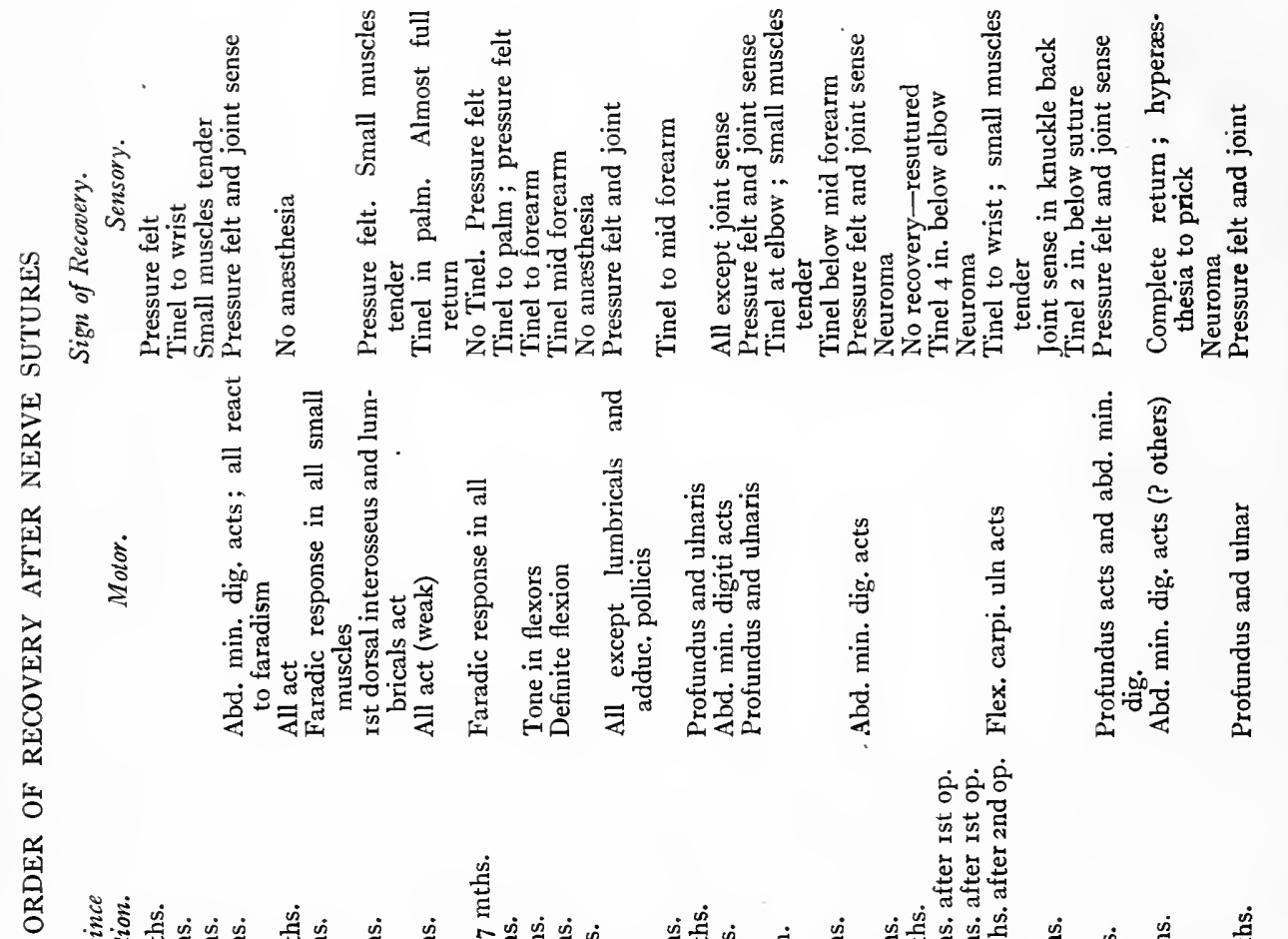

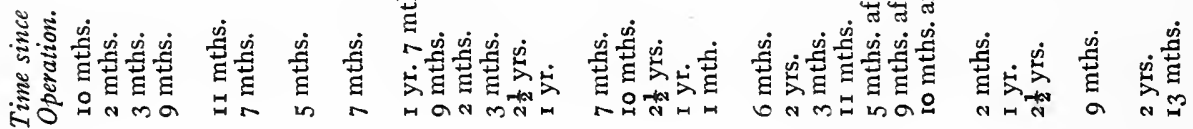
空

$\frac{\sqrt[n]{2}}{\frac{5}{2}}$

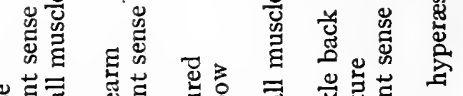

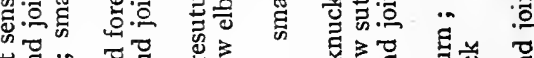

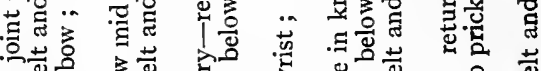

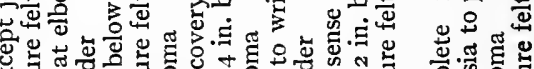

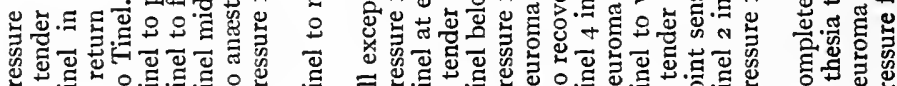

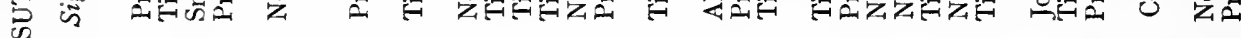

章

赔 

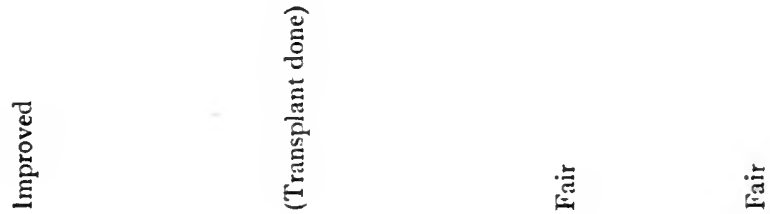

岂

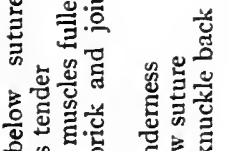

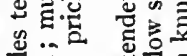

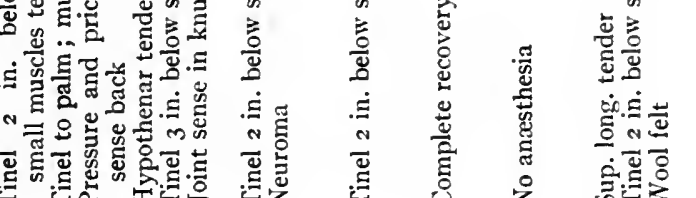

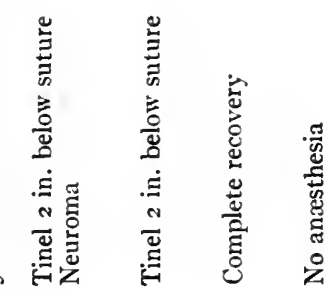

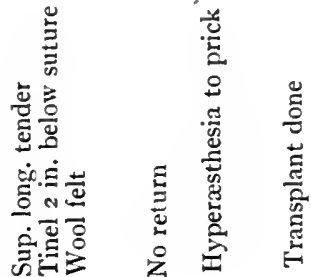<smiles>C#CC=C</smiles>

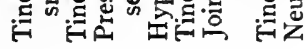
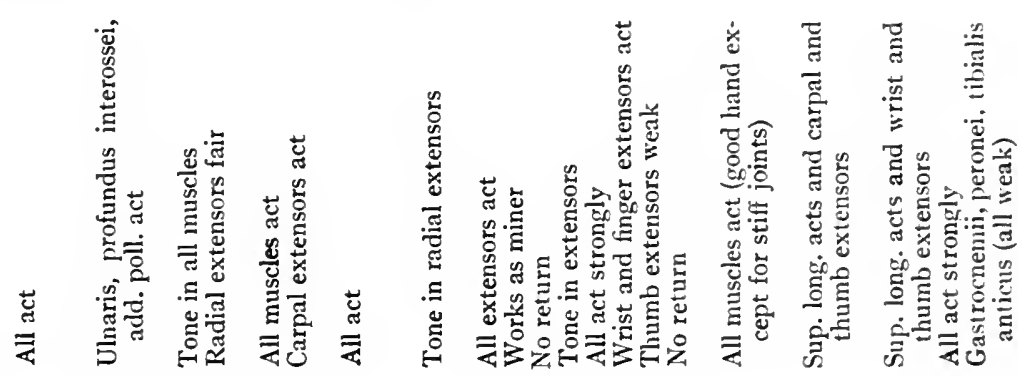

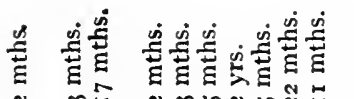

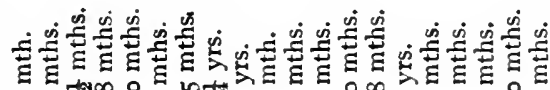

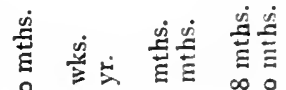

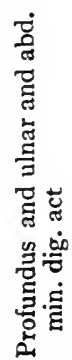

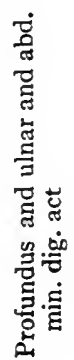
气

\section{.}

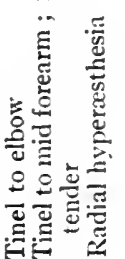

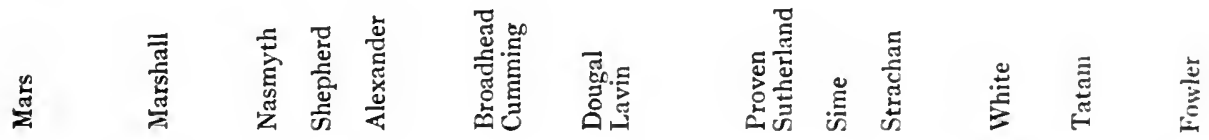
葛

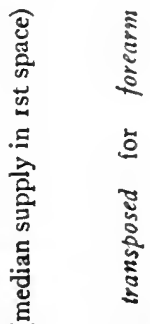

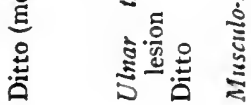

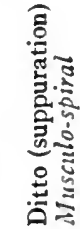

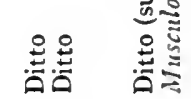

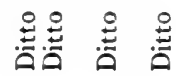

כ)

¿ूँ

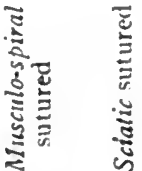




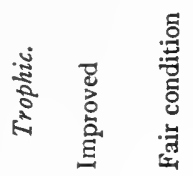

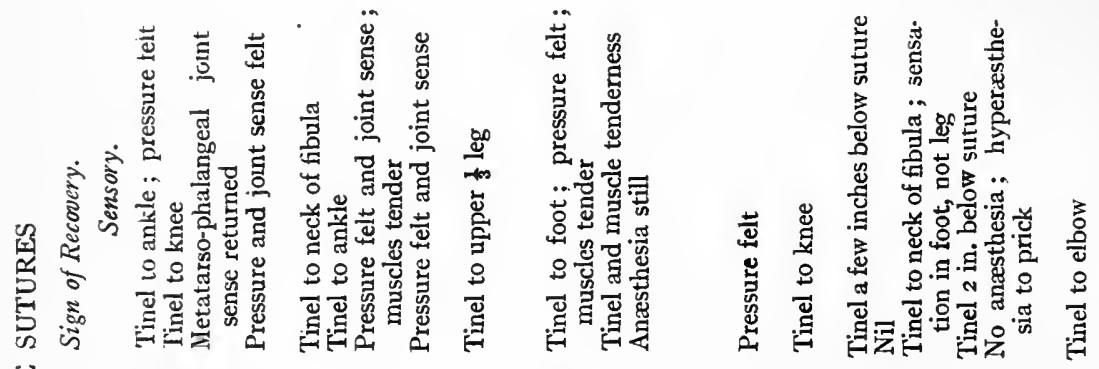

崚

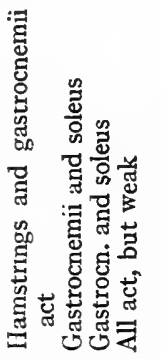

芯 芯

节 范

官

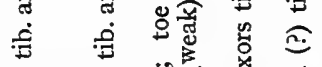

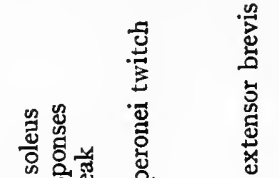

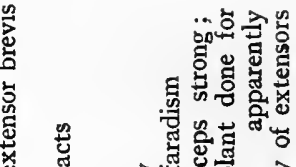

चี नี

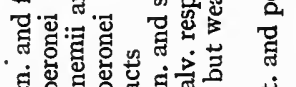

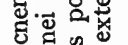

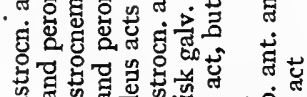

苍 的

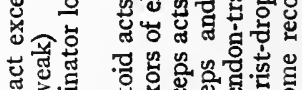

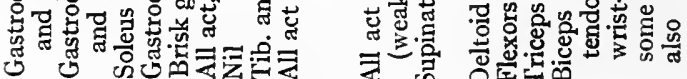

贸.

爱

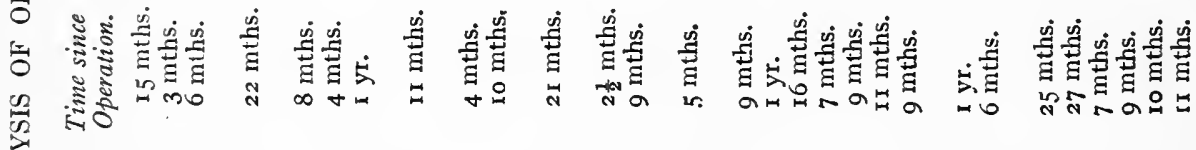

空

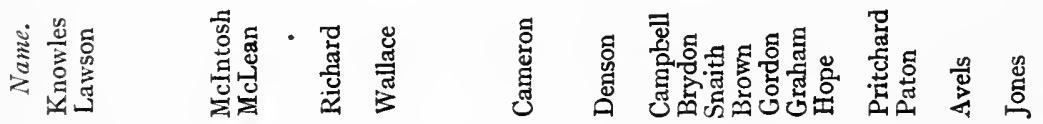

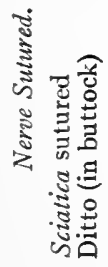

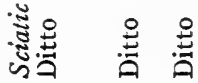

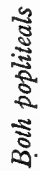

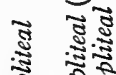

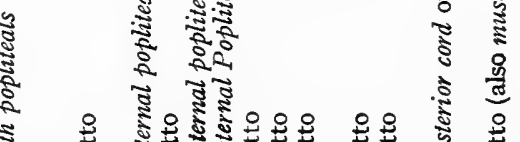

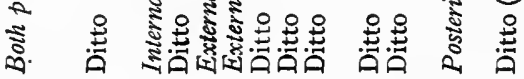




\section{AFTER-TREATMENT}

Some writers have advocated long and elaborate treatment of cases of nerve injury before operation, with a view to freeing tendon and joint adhesions and restoring the nutrition of paralysed muscles to make them receptive of returning nerve impulses, but it seems more logical to regard the operation itself as the preparatory treatment. For instance, many of the tendon adhesions are of a toughness which will only yield to the knife ; the peripheral joint adhesions are dependent on perverted trophic influences in the nerve and subside spontaneously when regeneration occurs, whereas the most vigorous treatment of other kinds fails to prevent relapse; similarly the wasting of the muscles is not prevented by electricity, while they fill out spontaneously as the nerve recovers irrespective of the amount of massage \&c. given. The only preliminary treatment which is important before a nerve operation is that directed to ensure free movement of the joints across which the nerve passes in the direction which will relax it for suture, e.g. the knee before exploration of the sciatic.

After-treatment in the case of nerve operations is also of value more in dealing with the complications than as a direct aid to regeneration. It must always be remembered that perfect anatomical regeneration may occur and yet the limb remain functionally useless, if there exist complications such as joint and tendon adhesions which are often more disabling than a nerve injury. In dealing with such, baths, massage, a gymnasium and electrical treatment are essential adjuncts to an operation.

Although it is difficult to find evidence that any form of after-treatment directly accelerates the process of nerve regeneration, yet it is frequently seen that the return of voluntary movement is delayed for want of muscle re-education, the patient remaining unconscious of the presence of regeneration until taught the use of his muscles again by a combination of electricity with suggestion. It is especially in paralysis of the intrinsic muscles of the hand and of the external popliteal that this is liable to happen. Patients have reported at Bangour more than a year after nerve-suture saying there was no recovery of their muscles, but when shown a faradic response in these, they at once began voluntary contractions, which increased rapidly in strength. These men might have been saved much time by re-education at an earlier stage. Electrical re-education cannot be begun until regeneration has occurred; however, treatment by Galvanism early after the operation serves to keep the patient interested in his limb, which is valuable. It might logically be expected to improve the circulation through the muscle, and so its 
nutrition; this is difficult to prove, for it certainly does not prevent considerable atrophy.

After-treatment has two other very important functions :

(a) to prevent separation of sutured nerve-ends by careless stretching of joints, when there has been considerable tension ;

(b) to prevent over-stretching of the weakened muscles by their opponents or gravity, for this may maintain the paralysis even after the nerve has recovered.

As regards the former point: if any neglect in the few weeks immediately following the operation permits a sudden strain on flexed joints with consequent rupture of the suture line, the operation has been done in vain, and no recovery need be looked for. Such an accident can easily occur when the splints are changed at the dressings, and therefore the surgeon should always remove the stitches himself and give the most careful instructions regarding the splints to the sister; it is well also to enlist the patient's interest in the matter. To ensure absolute safety, it was the practice in the Edinburgh War Hospital to allow no increase in the range of the joints, beyond that noted as safe at operation, until six weeks later; then gently to stretch them as much as they would yield without causing nerve-pain, and after this to trust to the patient's own choice. Although extreme flexion of three joints of the upper limb was resorted to in difficult cases, none was seen in which the full range was not ultimately obtained (apart from direct joint injuries); usually free movement was complete in 3-5 months, according to the degree of tension at operation. It is satisfactory, though not essential, to obtain the full range before the patient is allowed to leave hospital, as judgement based on the operative findings is required in deciding on the rate of increase permissible, while if the flexed position is allowed too long, habit may produce a functional contracture.

As regards muscle-overstretching, some neurologists now deny the importance of the continuous relaxation of paralysed or weakened muscles. Nevertheless, at Bangour one was impressed with the rapidity with which certain cases, especially those of wrist-drop and foot-drop, recovered after the application of a constant support, perhaps in the course of days, although they had made no progress for weeks or months previously with other forms of treatment. Similarly, the men who were conscientious about maintaining the relaxed position, were noticed to progress more rapidly than those who were careless. It is impossible to give figures in support of this; nevertheless, the impression was irresistible. Tendon-transplant cases, which in the early stages of one's experience were allowed to become over-stretched, formed striking instances of the sort, and recovered again with continuous support.

It is a good principle to support all muscles which would otherwise 
be over-stretched by the effects of gravity, until such time as voluntary power returns and does not diminish during gradual withdrawal of the support. The final result of tendon-transplant operations was found to depend largely on the rigid application of this treatment until re-education was complete.

In the case of mixed injuries, it is best to relax only that group which could be over-stretched by gravity. The moderate stretching of their opponents which results, seems in no way injurious. Complete median

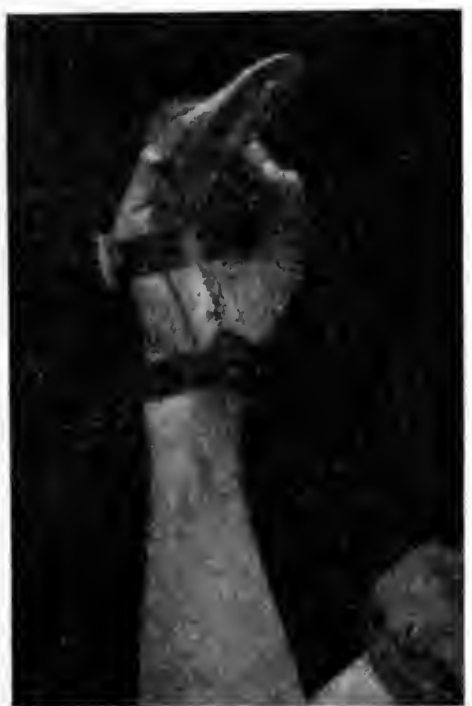

$a$.

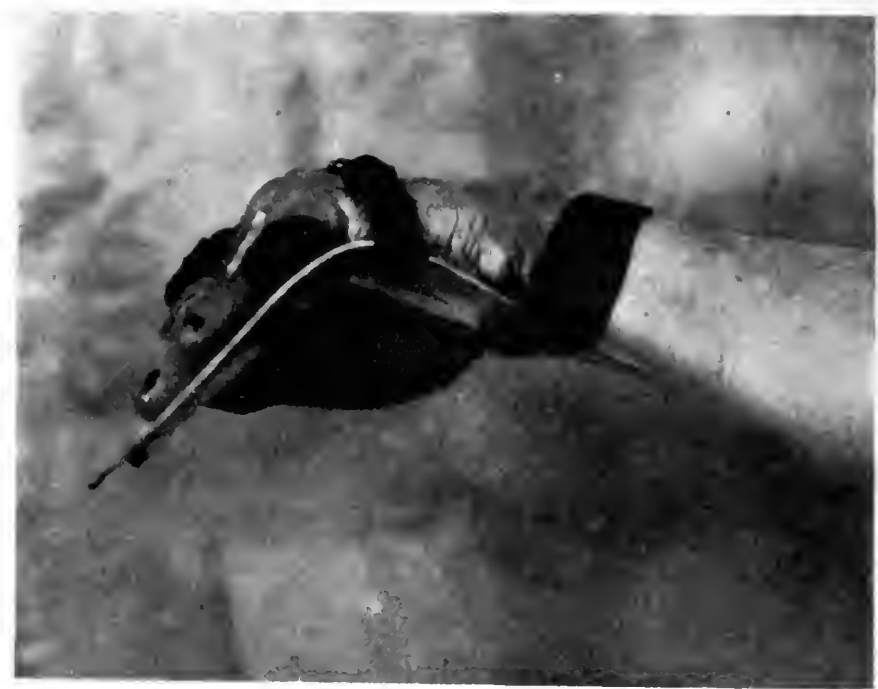

b.

Fig. 17.-Major Danforth's papier-mâché splint, reinforced by metal, for paralytic claw-hand.

and ulnar injuries really represent mixed injuries, for relaxation of the long flexors means over-stretching the intrinsic hand muscles which are extensors of the same joints. Neither group are appreciably affected by gravity. As these cases show a tendency to flexion contracture, they should not be splinted in flexion; sometimes the contracture must be overcome by splints; also the flattening of the hand ('ape-like') with loss of opposition of the thumb is usefully overcome by a small plaster splint. One was introduced by Col. Danforth, U.S.A., consisting of a square of plaster of Paris bandage folded into two, with the corner wedged in between the thumb and index finger, while the rest is wrapped round the first metacarpal and bandaged firmly to the hand to maintain the transverse arch.

With regard to the objection often brought forward that continuous 
splinting causes stiff joints, the matter is not yet proven. There were at Bangour uncomplicated musculo-spiral cases kept continuously on long cock-up splints till recovery occurred ten to eighteen months later, and having no stiffness of the knuckles, provided that daily massage was given and that the splint was shaped so as not to cause hyper-extension. On the other hand, unsplinted cases of median and ulnar injury and septic cases can be seen daily developing increasing stiffness up to a severe form, in spite of baths and massage. The best prevention and treatment of such was in our experience elastic traction, applied exactly in the line of the deformity or limitation, any grinding together of the joint surfaces

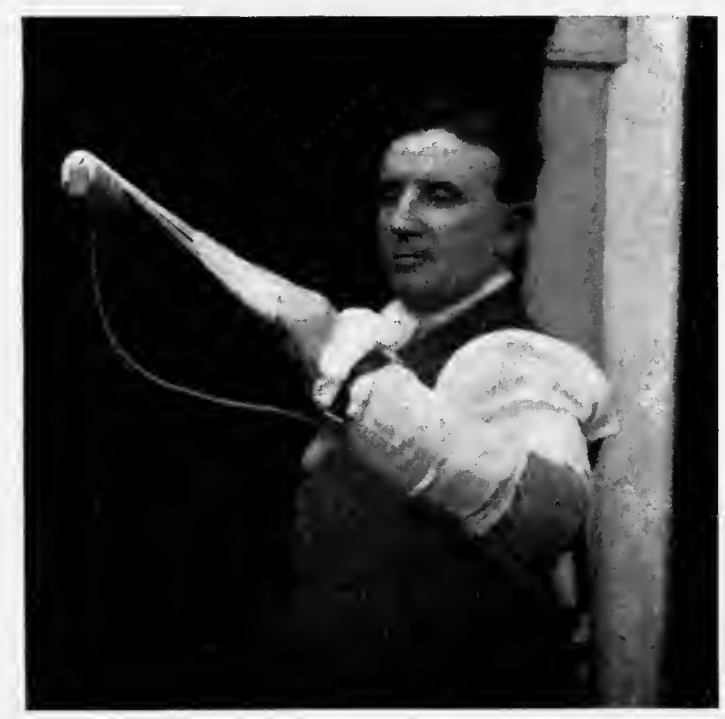

FIG. I8. - Traction-splint for contracted fingers and stiff knuckles; made of springy metal.

being carefully avoided. The splint was of springy metal of the 'banjo ' type, or else a plaster-cast with a wire out-rigger. A contracture could be treated equally well, and indeed simultaneously by this means.

In conclusion, it may be said again that it is for the prevention of contractures, and of stiff joints, and for the re-education of muscles during nerve regeneration that out-patient treatment has its value after operation. Once a stationary condition of the lesion is reached, outpatient treatment is only harmful, by turning the patient into a chronic invalid. He should be encouraged to face his disability frankly and to lay out his life in accordance with the limitations it imposes. That a fair decision may be reached in each individual case, it is most important that the patient should be re-examined at regular intervals by the surgeon 
who has seen the actual lesion at the operation. It should be remembered that although the restoration of a limb to the perfectly normal condition, can only be looked for in a minority of the cases, yet in war time twothirds of the men were able to do work useful to the community and sufficient to earn their livelihood, and the same would hold good in peace time, if economic conditions permitted. A very small interference with function suffices to incapacitate a man for specially skilled or very heavy work, but fortunately there are many useful occupations which are less exacting in this respect, and though the surgeon cannot hope to put many of his nerve cases into the former category, yet he may well render limbs which were useless suitable for the second. 


\section{OPERATIVE TREATMENT OF WAR INJURIES OF THE PERIPHERAL SPINAL NERVES}

\section{General Considerations.}

INJURIES to the Nerves of the Upper Extremity.

Brachial Plexus :

Exposure of Upper and Middle Trunks.

Exposure of Lowest Trunk.

Exposure of the Entire Plexus.

Nerves in Middle Third of Axilla.

Combined Lesions of the Nerves and Vessels of the Axilla.

Nerves in the Lower Third of the Axilla and Proximal Half of the Upper Arm.

Musculo-spiral Nerve in the Lower Half of the Arm.

Posterior Interosseous Nerve.

Ulnar Nerve :

In Lower Two-thirds of Forearm.

In Upper Third of Forearm, and its Transposition.

Median Nerve :

In Lower Two-thirds of Forearm.

At the Elbow and Upper Third of Forearm.

Tendon Transplantation for Median Paralysis.

Nerves of the Hand.

Injuries to the Nerves of the Lower Extremity.

Sciatic Nerve.

In Buttock and Upper Part of Thigh.

In Lower Half of Thigh.

External Popliteal Nerve.

Internal Popliteal Nerve.

Posterior Tibial Nerve.

Nerves of the Foot.

Anterior Crural Nerve.

Procedures wilen End-to-end Union cannot be Effected.

Nerve Grafting.

Nerve-flap Operations.

Bone Shortening.

Nerve Anastomosis. 


\section{GENERAL CONSIDERATIONS}

As the operative treatment of peripheral nerve injuries frequently entails extensive, and sometimes difficult dissections, it is essential that the operator should possess a thorough practical knowledge of dissecting-room anatomy. If he has not already acquired this knowledge, he should either take a short course in the dissecting-room, or be content to serve a preliminary apprenticeship as assistant to a surgeon who is constantly operating on these cases.

In abdominal operations, one assistant is generally sufficient, but in operations on the nerves of the extremities, three are generally required, namely, one to steady the limb, a second to swab the wound and secure the vessels, and a third to take charge of the retractors.

As the dissection may be a long one, the operator should sit rather than stand, and should have the limb supported at a convenient height.

It is not advisable to apply a tourniquet to the limb, as much time has to be spent in securing the bleeding points after its removal, and the tendency to subsequent oozing into the tissues is greater than when no tourniquet is used; morcover, the vessels often serve as a guide to the nerve and its branches. During the operation the wound should not be bathed with normal saline; the best lotion is the patient's own blood.

The knife should have the keenest possible edge, which should be bulged into a slight belly a little behind the point, and the handle should be sufficiently heavy to give the instrument the proper balance.

The dissecting forceps should have toothed extremities to enable the nerve to be picked up by gripping the sheath only, and the spring of the forceps should not be too strong, otherwise the muscles of the operator's thumb will soon become tired.

In using the knife, clean, rapid, and decisive cutting should be done with the belly of the knife rather than scratchy work with the point. Accurate suturing of the wound is greatly facilitated by scratching the skin at right angles to the direction of the incision; this is specially important, as the joint in the neighbourhood of the wound frequently requires to be flexed to prevent tension on the sutured nerve. While making the incision, it is an advantage to stretch the skin transversely between the forefinger and thumb of the left hand.

In making the skin incisions, it is hardly necessary to point out the importance of an accurate knowledge of the surface guides to the nerves. Should the original scar happen to be in the neighbourhood of the normal skin incision for exposure of the nerve, the skin incision should be modified so as to enable it to be excised. In this connexion, it may in certain cases be advisable to excise the scar at a preliminary operation, this procedure 
at the same time being a useful indication as to the presence or absence of latent sepsis.

The dissection to expose the injured nerve should err on the side of being too extensive rather than too limited, and the importance of exposing the nerve above and below the lesions in the first instance cannot be too strongly insisted upon. This is especially important when the nerve is embedded in or displaced by scar tissue or callus.

While the writer is careful to use gloves in all septic cases, he considers it a distinct asset to be able to dispense with them where rapid and delicate dissecting work is required; but the omission of gloves must not be attempted unless the surgeon has learned how to disinfect his skin and at the same time maintain its smoothness. Nor is it necessary in performing nerve dissections to protect the wound from the adjacent skin by clamping gauze to its cut cdges. By so doing, one only encumbers the field of operation unnecessarily, and by covering the surface-landmarks the difficulties of the dissection are greatly increased.

In freeing the nerve, the operator should conserve the branches as far as possible; the best way to avoid them is to know exactly where to expect them.

During the dissection it is often a help to flex the limb a little so as to take the tension off the nerve and allow also of the retraction of the adjacent structures. The nerve should, of course, be handled with the greatest possible gentleness. The advantage of toothed dissecting forceps has already been referred to.

As already stated, the nerve is often adherent to or surrounded by, and sometimes considerably displaced by, an abundance of cicatricial tissue which binds it down to the adjacent muscles, which have themselves been more or less extensively injured. The dissection, therefore, must frequently be carried wide of the injured nerve in every direction, and it is most important that the muscles and tendons, as well as the nerve, should be freed from all cicatricial tissuc. When the injury involves one of the nerves of the forearm, it may be necessary to combine the suturing of the nerve with a repair or transplantation of one or more of the adjacent tendons.

The nerve lesion itself must now be dealt with, but, before this is done, all the vessels should be tied so as to get rid of the forceps. Next, the degree of excitability of the nerve to the faradic current should be ascertained, and, while this is being done, the limb should be carcfully exposed distal to the lesion; hence the disinfection of the skin should be continued to its extremity. Attention is called to the possibility, from spread of the current, of mistaking contraction of opposing muscles for movements of the muscles that are being watched. Again, although stiffness of the fingers should, as far as possible, be dealt with before 
operating, this factor may introduce a difficulty in eliciting or translating the electrical findings.

If the lesion be a complete one, the bulbous or cicatricial extremities should be amputated with a very keen-edged knife, the nerve being meanwhile supported; and enough of the stump should be removed to expose apparently normal nerve bundles throughout the entire face of both stumps. If the nerve has not been completely divided, all cicatricial tissue should be removed from its sheath, after which the nerve should be carefully palpated to ascertain its degree of induration, and whether or not there are any localized cicatricial or gliomatous nodules in its substance.

If the nerve has been completely divided, the cicatricial, or neuromatous, stumps should not be amputated until all is ready for suturing. In amputating the ends, the nerve should be carefully supported so that a clean cut may be made, and for this purpose a very keen-edged knife should be used.

If, on stimulating the nerve above the thickened or bulbous portion, it is found that the muscular response is fairly good, it is often advisable to incise the involved portion longitudinally in one or two places; if, on the other hand, the faradic response is feeble, an exsection should be done and enough removed to expose nerve fibres free from all scar or neuromatous tissue. In dealing, however, with partial lesions of nerves, no hard and fast rules can be laid down; each case must be judged on its own merits. Personally, if in doubt, the writer is inclined to favour exsection, provided always he is satisfied that the two stumps can be brought into apposition without tension after the whole of the damaged segment has been removed.

With regard to closure of the wound, subcutaneous sutures are unnecessary, and their introduction only serves to prolong the operation. It must be remembered, however, that wounds in the long axis of the limb may give rise to a little tension, so that the sutures should be left in for ten days or so. Silkworm gut is the best material to use.

With regard to drainage, it is seldom necessary to introduce a tube, especially if interrupted sutures are employed, when all that is needed is to leave an interval between one or two of the stitches sufficient to admit of the escape of the blood-stained serum.

When flexion of the adjacent joint is necessary to take the tension off the nerve, the limb should be carefully splinted, and the first dressings should not be left entirely to the nurses. It is hardly necessary to point out that those in charge of the after-treatment should not be in too great a hurry to straighten the limb.

Where joints have to be flexed to allow of approximation of the segments in cases of suture, the question of how soon after operation 
extension should be started and completed is important. The exact time after operation at which firm union occurs at the suture-line is not definitely known, and, therefore, in this connexion, it is better to err on the side of safety. The pain experienced by the patient when tension is put on the nerve is a useful indication as to the rate at which the extension of the flexed joint should be proceeded with. It should be pointed out, however, that if extension of the joint is begun before the new axones have grown into the distal segment, rupture of the suture is possible without pain. For example, the usual practice at the Edinburgh War Hospital in the case of a sciatic nerve suture requiring knee flexion to an angle of $90^{\circ}$ for relaxation purposes is to wait for a period of four to six weeks from the date of operation before commencing to extend the knee ; gradual extension, a few degrees every week, is then proceeded with, employing two months more before obtaining complete extension. In no case has limitation in extensibility occurred as a result of flexing joints for such periods. A retaining splint is advisable in these cases, as extension of the joint may otherwise inadvertently take place.

Increased experience has convinced the writer that the tendency has been to delay operation too long. We are justified in delaying if there is definite evidence that improvement is taking place, and this is more likely to happen if the nerve has been contused as a result of a fracture, or if the symptoms are due to the pressure of callus. In such cases the improvement is progressive, and often ends in complcte recovery. In cases, however, where the nerve has been directly injured by a bullet or piece of shrapnel, it is a mistake to wait, although the lesion may be only partial. Many of these partial lesions are attended with severe pain, with aggravated trophic disturbances and with cicatricial or reflex contractures. In such cases valuable time is wasted in waiting for a recovery, which in the end is only very partial. The operation can do no harm, the wound is healed in a fortnight, and the exploration enables us to ascertain the exact nature of the lesion. The pain, the trophic changes, the reflex spasm, and the contractures often rapidly disappear. In short, the operation will not only expedite the recovery, but will at the same time render it more nearly complete. The majority of authors recommend a delay of from three to six months to avoid the risk of lighting up latent sepsis. Unfortunately, even a delay of six months is no guarantee against this danger. For some time past our practice has been to apply firm, and then heavy, massage to the wound as soon as it has completely healed. If this causes no inflammatory reaction, operation may be undertaken within two or three weeks from the date of healing.

While in some cases the freeing of the nerve is a very simple matter, in others it may be difficult on account of adhesions to vessels or from the fact that the nerve is embedded in dense scar tissue or bone callus. 
It occasionally happens, too, that the nerve has become considerably displaced from its normal course, and when this is the case, the operator may divide it accidentally, unless he is careful to expose it on the proximal and distal side of the lesion before dealing with the lesion itself.

In cases in which there is no response to the faradic current clinically, the nerve may sometimes be traced intact through a long stretch of dense scar tissue or along a deep groove in callus. After the nerve has been completely freed, it is carefully examined by inspection, by palpation, and by the faradic current (see p. I49).

Inspection may reveal the nerve to have an almost normal appearance, and yet the faradic response may be very feeble or even entirely absent. In such cases the nerve is generally somewhat atrophied-looking, and on palpation the impression gained is that the sheath is imperfectly filled, giving the nerve a ribbon-like, rather than a cylindrical, feel; another important feature of these cases is that the condition above described may extend for several inches. The indications here are to be content with freeing the nerve rather than doing a resection, and especially is this the case when the dissection has revealed evidence that the nerve has been compressed. In these cases the extent of the lesion and its imperfect delimitation make the more radical operation of resection unsuitable, at any rate until neurolysis has been given a chance.

More frequently, the freed nerve will be found to be thickened, and more or less firm and indurated, and this may take place either in a uniform or nodular manner. Not unusually, two nodular thickenings are separated by a short interval in which the nerve is shrunk. This generally signifies that the nerve fibres have been severely contused or ruptured, while the sheath has remained intact. In other cases, the lesion is represented by an elongated or more or less fusiform thickening of the nerve, which is generally, though not invariably, of a firmer consistence than the normal nerve. From the operation point of view, it is important to remember that this thickening may be almost confined to the sheath, and when this is the case, the thickening is generally secondary to an extravasation of blood around the nerve or to the spread of suppuration along the perithecal cellular tissue. In one instance, there were three thickenings of the sheath of the sciatic, each corresponding to the position of a drainage tube. It is necessary to remove the thickened sheath with scissors curved on the flat before undertaking a more detailed investigation of the nerve. At this stage the question will often arise as to whether the operator is to be content with merely freeing the nerve, or whether he should proceed to do a resection followed by end-to-end suturing. This is often a very difficult question, especially when the faradic current elicits a fairly good response in some of the muscles supplied by the nerve below the lesion. Out of 500 nerve opera- 
tions performed at the Edinburgh War Hospital, the nerve has been resected in practically 50 per cent. of the cases. In a few cases, patients whose nerves were merely freed have had to have the lesion resected at a subsequent operation on account of the insufficiency or absence of improvement. Looking back on these cases in the light of present experience, the writer is satisfied that he would now do a resection in several of the cases in which neurolysis was done, and in a few cases he would now do a neurolysis where formerly he had done a resection. Every case must be treated on its own merits. For further information the reader is referred to the chapter on the Diagnosis and Lesions, as well as to that dealing with the electrical examination of the nerve. But, while it would be useless to attempt to lay down hard and fast rules which would apply to all cases, a few words may be said with regard to the more doubtful ones. If, after examination by inspection, palpation, and the faradic current, we are still in doubt, the next thing to do is to incise the injured area longitudinally. Should the cut surface reveal nothing but fibrous scar tissue, resection is indicated; if, on the other hand, bundles of nerve fibres can be traced through the thickened segment or through the neuroma, it is not usually necessary to resect the nerve. In a case where certain fibres are destroyed, it is necessary to consider the relative value of the two sets, and if those destroyed are of vital importance, it may be wise to sacrifice the intact fibres as resection gives the only chance of adequate recovery. A good example of this is in a lesion of the median in the upper arm where the function of the index finger and thumb is lost, while that of the pronator teres, the flexor carpi radialis, and palmaris longus is present ; resection would be advisable in such a case. On the other hand, if the index finger and thumb were functioning and the pronator teres and flexors of wrist gone, a policy of conservatism would be best ; in the latter case the retention of sensibility in the median distribution would be a preponderating factor in favour of conservatism. Sometimes it is advisable to make a second longitudinal incision into the nerve; by incising the shcath, tension is relieved and harmful pressure is gradually removed from the nerve fibres, and this procedure, along with the neurolysis, may be sufficient to initiate recovery of function. The musit difficult cases are those in which the cut surfaces of the lesion present a firm, pinkish, homogeneous appearance without any macroscopic: fibrillations. Although the microscope often reveals the presence of young axis cylinders in the neuroma, they have failed in many cases to entır the nerve on the distal side of the lesion. Such thickenings should, therefore, bc resected, and especially is this the case when the stumps can be brought into apposition without having to divide important motor branches.

In partial lesions of the nerve, where only a portion of the circum- 
ference has been injured, the question is less complicated, as the unilateral neuroma may often be excised without having to resect the whole thickness of the nerve. To prevent undue kinking in cases of partial resection and suture, it is often an advantage to split the nerve longitudinally before suturing, so that the uninjured portion runs a wavy course instead of being sharply angled. This is specially advantageous in dealing with unilateral injuries of the sciatic nerve. If considerably more than half the fibres have been divided, it is wiser to do a complete resection to enable the partially divided nerve to be accurately sutured.

When the nerve has been completely severed and the ends have become separated, or when a section of the nerve has been destroyed so that a wide gap intervenes between the stumps, the aim of the operation should be to effect an end-to-end suturing of the prepared stumps, successive slices being removed in a proximal and distal direction respectively until healthy, or at any rate almost healthy, nerve bundles are exposed. In slicing the nerve it is important that a sharp knife be used and the sheath of the nerve segments be held firmly and accurately by tooth forceps, to avoid making anything but a clean transverse section. In determining how much to resect, special attention should be paid to the condition of the fibres on the proximal side of the lesion; it is important when trimming the stumps to attack the proximal end first, because if a doubtful part has to be left in order that the ends may be approximated, it is better that it should be in the distal segment. Before slicing the bulbs, however, the operator, for the reason stated later, should satisfy himself that after having done so, the ends can still be approximated. Before suturing the prepared stumps, all scar tissue should be removed, muscles and tendons should be freed from adhesions, and, if necessary, sutured or otherwise repaired. Again, before suturing the stumps, all bleeding should be carefully arrested and hæmostatic forceps removed. In some cases it is advisable to sew up part of the skin incision before carrying out the suture of the nerve, e. g. in lesions in the axilla where adduction of the arm is necessary to obtain coaptation of the stumps.

Before, however, the nerve is sutured a stitch should be introduced into corresponding aspects of the proximal and distal portions of the sheath, an inch or two from the stumps; these are for the purpose of acting as temporary guides to prevent axial rotation of one stump upon the other while they are being sutured. In cases where there was physical continuity before resection, the guides should be inserted before the nerve is divided.

With regard to the suturing of the stumps themselves, either fine linen thread, fine silk, or thin catgut may be used. The writer prefers the No. I6o linen thread supplied by Messrs. Turnbull \& Wilson, drapers, Edinburgh. An objection to chromic catgut is the irritation produced 
by the chromic acid. The sutures should take up the sheath only, and for their introduction either a fine,sewing needle or a fine curved needle may be used according to the option of the operator. The sutures need not be more numerous than is necessary to retain all the nerve bundles within the sheath, and the amount of tension of each suture only moderate; usually from four to six sutures are sufficient for a nerve the size of the median. Only enough of the sheath should be included in the suture to ensure an efficient grip being obtained ; if too much is included, inversion is very liable to occur. It is recommended that the first two sutures be introduced at opposite aspects of the circumference; after tying them, they are left long so that they may be used to steady and rotate the nerve while the remaining sutures are being introduced.

Special ring forceps or a small size of Lane's fenestrated tissue forceps are convenient instruments for retracting or steadying the nerve. Fine toothed forceps are employed to take up the edges of the sheath while the sutures are being introduced.

Some opcrators favour the envelopment of the sutured stumps in a sheath of fat or fascia lata, others prefer to slide a portion of a vein over the suture line, while others again make use of Cargile's membrane. The idea is to prevent the nerve from becoming adherent to the adjacent tissues and to prevent also the ingrowth of fibrous tissue between the sutures. The writer is of opinion that all such devices only serve to set up the very mischief they are intended to prevent. The few cases he has operated on in which one or other of these methods has been employed at a former operation, have only served to confirm this opinion. All that is necessary is to see that the sutured nerve is not left in contact with scar tissue, bare bone, separated periosteum, or the deep surface of the skin opposite the suture line. Healthy muscle, fat, or loose cellular tissue form the best and most natural bed for a nerve.

We have now to deal with a case in which the injury to the nerve has been more extensive, and where there is considerable doubt as to whether a direct end-to-end suture can be effected. It is safe to state that those who have had a large experience in operating on war injuries of the peripheral nerves would now be able to obtain an end-to-end suture in cases in which, from insufficient experience, they were, in the early part of the war, unable to do so.

Let us take, by way of example, extensive destruction of the median nerve in the middle third of the upper arm. As this nerve gives off no branches until it reaches the elbow, it may straightway be freed from this level up to the pectoralis minor muscle. This having been done, the next step is to see if the ends can be brought into contact after fully flexing the clbow, and at the same time bringing the arm to the side. Should this not suffice, the next thing to do is to stretch the two portions 
of the nerve; this is done by grasping the bulbous ends with artery forceps and making gradual traction first on the one stump and then on the other. It should be observed that it is of no use attempting to lengthen the nerve by traction unless it has been completely freed, and the greater the length to which it is freed, the more it can be lengthened. Observe, too, the importance of making the traction before the bulbs are removed. Should the ends still fail to meet, or rather should the bulbs still fail to overlap so that the stumps when freshened would not meet, a further gain can be got by mobilizing the pronator teres muscle, so that the branches which are given off by the median in the upper part of the forearm may be isolated and stretched along with the main trunk. The mobilizing of the pronator teres and the division of the tendinous arch of the flexor digitorum sublimis muscle also help to shorten the course of the nerve, because when the elbow is flexed the nerve instead of being tacked down comes forward like a bowstring.

If this fails, what next? Fortunately we have still another procedure, and that a valuable one, which we can draw upon, and that is to suture the bulbous ends together, or as nearly together as they will come, using thread strong enough to stand the strain of the traction. While this is being done the limb must be held with the arm well adducted and the elbow fully flexed. A fortnight later the elbow is gradually extended so that from six to eight weeks after the operation full extension is reached. If still more traction is deemed advisable, the limb may be gradually abducted from the side by means of a splint provided with a lengthening screw. After the arm has reached the horizontal plane, or nearly so, the nerve is again cut down upon, when it will be found that it has become so stretched that with the arm again adducted and the elbow flexed, the bulbs can be removed and the freshened stumps approximated. This may with advantage be termed the Two-stage Slow-stretching operation. In the opinion of the writer this is a valuable procedure which should be resorted to in preference to such alternative operations as the nerve flap operation, nerve grafting, nerve anastomosis, bone shortening, or bridging with cat-gut, calves' arteries, \&c. (Fig. I9.)

To illustrate another principle, we will suppose we are dealing with an extensive lesion of the median nerve in the middle of the forearm. The important point here is to dissect out and follow both proximally and distally all the motor branches which the median gives off at the bend of the elbow and upper part of the forearm. The details of the operation will be given later on (see p. I0o). When this has been done, the trunk of the nerve is freed as far above the elbow as necessary. By applying traction to the upper bulb, it will be found that the extensively freed portion of the nerve proximal to the lesion can now be pulled well down into the forearm, because the dissected out branches of the median now 
offer no hindrance. Their origins become approximated towards their points of entrance into the muscles; in other words, these branches become relaxed instead of stretched when the median is pulled downwards. This is all the more important as, owing to the fixation of the median at the wrist, very little can be gained by upward traction of the nerve distal to the lesion. We may therefore lay it down as a principle that, in attempting to obtain an end-to-end junction after an extensive nerve resection, full advantage should be taken of a long proximal dissection, and while the main trunk is being freed, any motor branch or branches which are encountered must be freed as far as possible in both

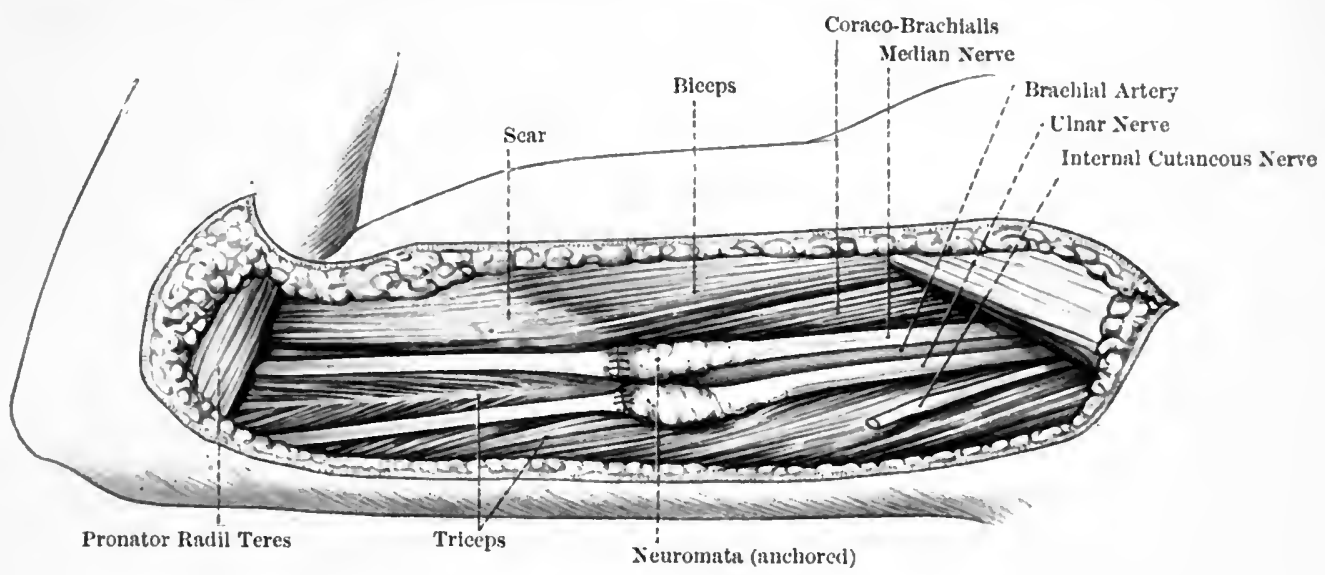

FIG. 19.-Operation for extensive lesions of the median and ulnar in the arm. The nerves felt hard for some distance above the neuromata, so that if all the unhealthy part had been resected, end-to-end suture would have been impossible. Therefore the neuromata were anchored at the first operation with the joints flexed. After a course of gradual stretching of the joints (and indirectly of the nerves), a second exploration was made and, after resection of the unhealthy parts of the nerves, end-to-end suture carried out with the joints again flexed.

directions. In this connexion, attention should be drawn to the anatomical fact that the motor branches which are furnished to limb muscles which belong to the same group, almost invariably arise close together from the parent trunk; moreover, by dissecting upwards the individual branches along the trunk (by splitting its sheath), they are generally found to group themselves into a common bundle which can be dissected still farther upwards. These statements apply not only to the branches of the median in the upper part of the forearm, but also to the corresponding branches of the ulnar and still more so to the branches of the musculo-spiral to the triceps, and best of all perhaps, to the branches of the sciatic which go to supply the hamstring muscles, with the exception of the branch to the short head of the biceps, which is explained by the 
fact that morphologically this muscle represents a displaced portion of the gluteus maximus muscle.

Limbs which have been flexed in order to allow of the approximation of the divided ends of the nerve must be carefully splinted. To enable the joints to be gradually extended, hinged or bendable splints are an advantage, and the splints should be retained until complete extension of the joint has been reached; otherwise the stumps may be inadvertently torn apart at the suture line. The special splints used will be referred to when dealing with the individual nerves.

\section{INJURIES TO THE NERVES OF THE UPPER EXTREMITY}

\section{BRACHIAL PLEXUS}

Surgical Anatomy. It will conduce to a clearer understanding of the operative procedures called for in dealing with the manifold lesions which may be met with in war-injuries of the brachial plexus, if attention be directed in the first place to the more important points regarding its surgical anatomy. (Fig. 20.)

A description of the mode of formation of the plexus will be found on p. 3. Speaking surgically, the anterior and middle scalene muscles constitute the chief guides to the cervical portion of the plexus. The roots (anterior rami) of the plexus emerge from behind the lateral border of the scalenus anterior, while the three trunks and their divisions rest upon the scalenus medius. Both these muscles, along with the plexus and the third part of the subclavian artery, are covered by a thin but definite layer of fascia, which is a lateral continuation of the prevertebral layer of deep cervical fascia. This fascia must be divided before the component parts of the plexus can be defined, freed, and separated from one another.

The dissection to expose the upper part of the cervical portion of the plexus, namely, the anterior rami of the fifth and sixth cervical nerves, their union a little external to the scalenus anticus to form the upper trunk and its anterior and posterior divisions, is a comparatively simple matter; exposure of the lowest trunk, formed by the anterior rami of the eighth cervical and part of the first thoracic nerves requires, on the other hand, a deep and delicate dissection.

The suprascapular nerve, which springs from the lateral border of the upper trunk, $I \frac{1}{2}$ in. above the clavicle, passes downwards, outwards, and backwards to disappear behind the clavicle close to the anterior border of the trapezius. If, therefore, we follow the upper trunk of the plexus towards the clavicle it appears to end in three nerve strands, namely, its two divisions and the suprascapular nerve. The anterior 


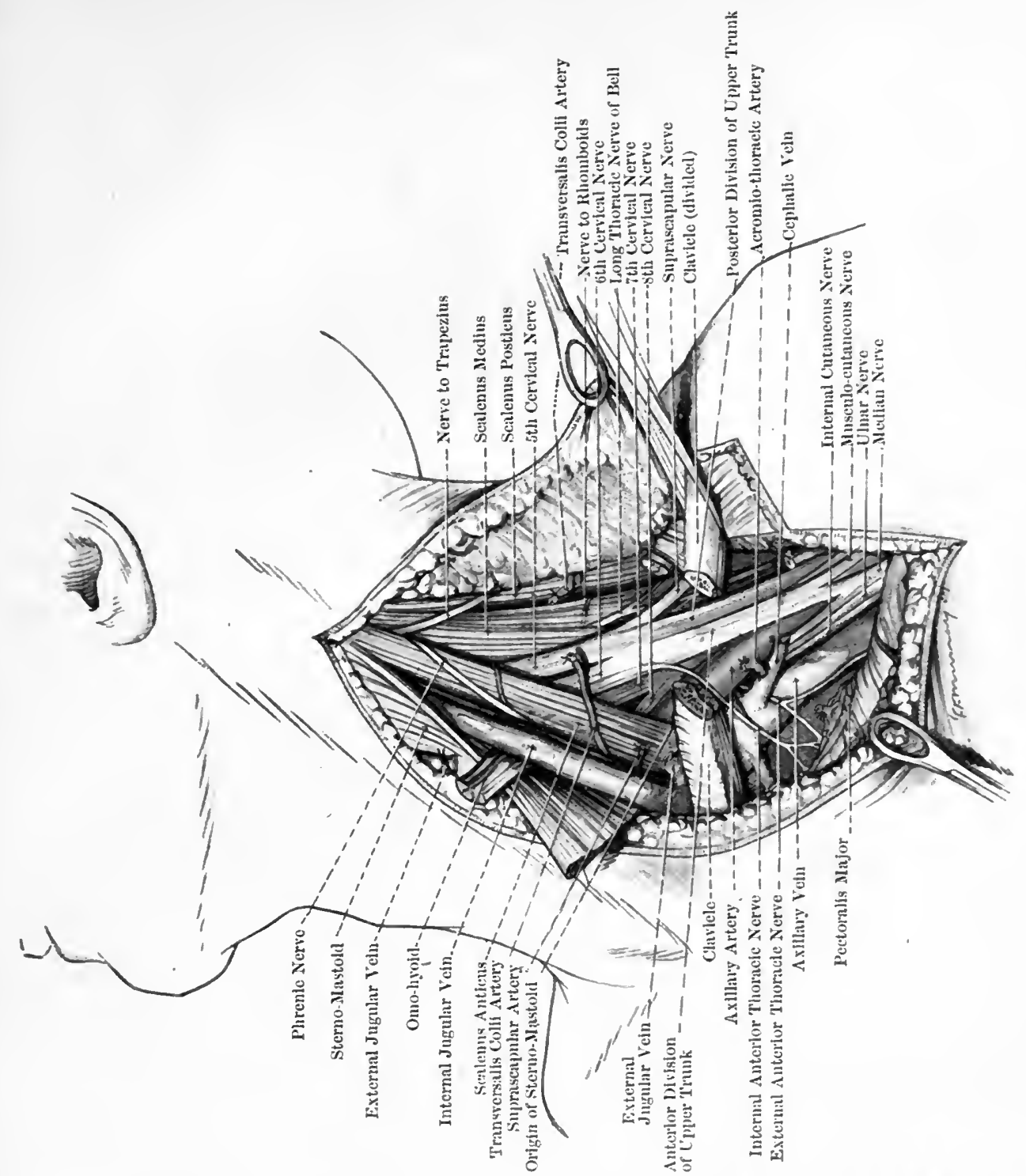

廿.

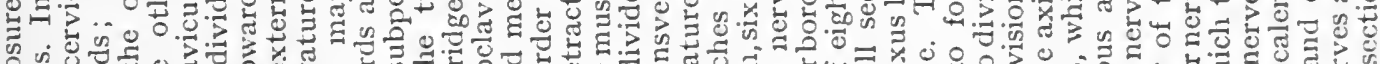
\% 0.

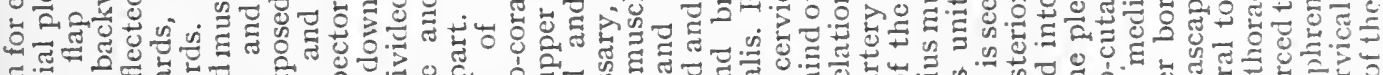

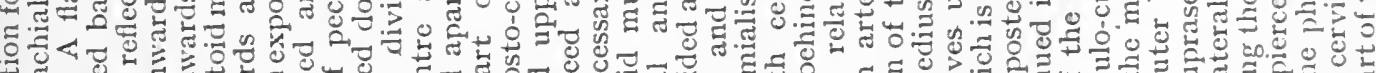
焉.

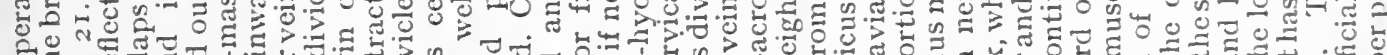

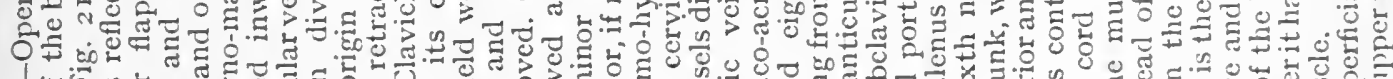

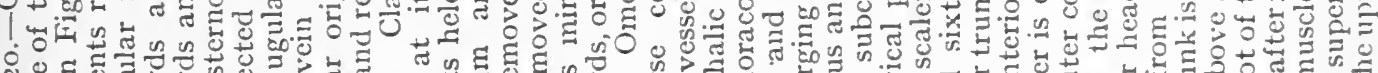

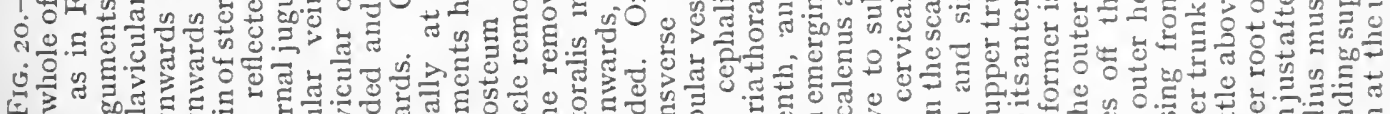

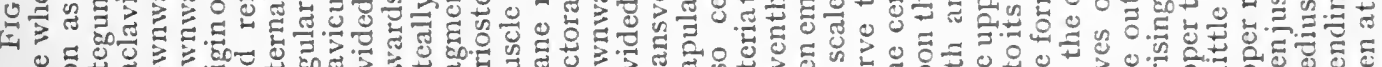

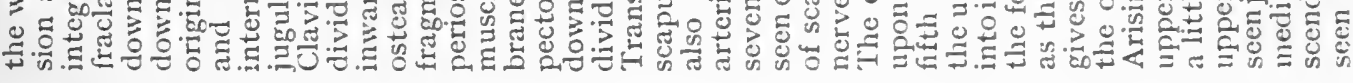


division helps to form the outer cord of the plexus, and furnishes the fibres to the external anterior thoracic nerve, the musculo-cutaneous, and the outer head of the median. Electrical stimulation of this division causes contraction of the upper part of the pectoralis major; the flexors of the forearm; the pronator teres, the flexors of the wrist (with the exception of the flexor carpi ulnaris), and the flexor digitorum sublimis. The posterior division helps to form the posterior cord of the plexus; stimulation of it causes contraction of the muscles inserted into the proximal half of the humerus, with the exception of the supraspinatus and infraspinatus muscles which are supplied by the suprascapular nerve and pectoralis major as noted above; also contraction of the supinator brevis muscle.

The lowest trunk of the plexus (formed by the anterior rami of $8 \mathrm{C}$. and I T.) lies deeply at the root of the neck, wedged in between the third part of the subclavian artery and the upper surface of the first rib. As shown by Wood Jones, this nerve trunk often takes as large (and sometimes even larger) a share in the formation of the subclavian groove on the rib as does the artery itself. To expose this trunk the third part of the subclavian artery must be freed and retracted downwards and inwards. When this has been done, note that the anterior ramus of the first thoracic nerve joins that of the eighth cervical at the inner border of the first rib just in front of the inner edge of the insertion of the scalenus medius muscle. Note also that both these rami lie in direct contact with the postero-lateral aspect of the cervical pleura.

The anterior division of this lowest trunk conveys fibres to the internal anterior thoracic nerve, to the inner head of the median, and to the ulnar nerve; hence when stimulated it causes contraction of both pectoral muscles, of the deep layer of muscles of the front of the forearm (flexor digitorum profundus, flexor pollicis longus, pronator quadratus), and of all the intrinsic muscles of the hand supplied by the ulnar nerve. The posterior division helps to form the posterior cord of the plexus, and when stimulated it causes contraction of the extensors of the wrist, fingers, and thumb, and to some extent also of the triceps.

The middle trunk, formed by the anterior ramus of the seventh cervical alone, is smaller than the upper and lower trunks, and is to some extent overlapped by them, so that it does not come fully into view until they have been retracted upwards and outwards and downwards and inwards respectively. The anterior division of this middle trunk helps to form the outer cord of the plexus, and when this division is stimulated it causes contraction of some of the fibres of the pectoralis major, of the coraco-brachialis, of the deep forearm muscles, and of those intrinsic muscles of the hand which are supplied by the median nerve. The posterior division contributes to the formation of the posterior cord 
of the plexus, and its stimulation causes partial contraction of the triceps along with contraction of the extensors of the wrist, thumb, and fingers.

Of the supraclavicular branches, the suprascapular has been already referred to; it is a useful guide to the upper trunk. If, along with an injury of the plexus, the muscles supplied by this nerve are paralysed, we know that the lesion involves the upper trunk; and if the rhomboid muscles are also paralysed, we know that the highest root of the plexus is involved, because this nerve springs from the outer border of the anterior ramus of the fifth cervical nerve before it joins the sixth. It emerges from the anterior surface of the scalenus medius a little above the highest root of the nerve to the serratus magnus and passes downwards and outwards upon this muscle for a short distance before it disappears from view by passing beneath the anterior border of the levator anguli scapulæ muscle.

The three roots of the long thoracic nerve spring from the anterior rami of the fifth, sixth, and seventh cervical nerves close to the intervertebral foramina. These roots supply respectively the upper, middle, and lower portions of the serratus magnus muscle. The upper two roots emerge upon the anterior surface of the scalenus medius muscle, while the lowest passes in front of it. All these roots are comparatively slender, so that it is not difficult to understand how an isolated paralysis of the serratus may be met with as a result of laceration, severe bruising, or overstretching of the scalenus medius muscle. Thus formed, the trunk of the nerve descends behind the plexus, which must be retracted downwards and inwards to expose it. While the nerve may be injured along with the plexus as it courses behind it, serratus paralysis as part of the syndrome of a brachial plexus lesion points rather to the lesion having involved the roots of the plexus, and this is all the more indicated if the rhomboid muscles are also paralysed.

While the main root of the phrenic is derived from the fourth cervical nerve, it generally receives a small root from the third and fifth cervicals. In extensive lesions of the brachial plexus, especially when the upper runk is involved, it is not uncommon to find the phrenic either embedded in scar tissue or completely severed. When the nerve is intact, electrical stimulation of it causes a bulging of the corresponding half of the epigastrium. Paralysis of one half of the diaphragm, the result of injury to the phrenic, is at once detected on $\mathrm{X}$-ray examination by the imperfect descent of the muscle on the paralysed side. Where the nerve descends upon the scalenus anticus muscle beneath the prevertebral fascia it is overlapped by the lateral border of the internal jugular vein, and is exposed therefore when this vein is mobilized and retracted inwards.

The external and internal anterior thoracic nerves supply the pectoral muscles; they spring from that part of the plexus which lies behind the 
clavicle, the external nerve from the inner border of the outer cord, the internal from the inner border of the inner cord; hence the pectoral muscles draw their nerve supply from all the roots which go to form the plexus. These two anterior thoracic nerves come into view in operations on the plexus which call for division of the clavicle and exploration of the upper axilla.

The details of the operative procedures which may be called for in dealing with war injuries of the brachial plexus will vary with the nature and level of the lesion, as well as with its extent and complications. It is necessary in the first place to describe the normal dissection for the exposure of the whole supraclavicular portion of the plexus.

With regard to the position of the patient, the shoulders should be elevated by putting a sandbag between the shoulder blades, and the head placed so as to stretch out the lateral aspect of the neck. A nurse should be detailed to keep the shoulder depressed by pulling on a clove hitch applied to the wrist, the ends of the bandage being sufficiently long to keep her well out of the way of the operator.

There are two incisions which may be regarded as 'normal', namely, the single oblique incision and the L-shaped incision. The former should begin rather above the middle of the posterior border of the sternomastoid muscle and end a little internal to the coracoid process. If it is found necessary to follow the plexus into the axilla, the incision is prolonged downwards as far as may be necessary. (Fig. 2I.)

The L-shaped incision is placed with its upper limb parallel, and a little internal to the posterior border of the sternomastoid, while its lower limb runs horizontally outwards a little below and parallel to the middle two-fourths of the clavicle. The writer prefers this latter incision because it gives free access to all parts of the plexus and allows of free removal of scar tissue-an important point when the plexus has been injured by shrapnel or shell-casing, which, besides injuring the plexus, has caused considerable laceration of adjacent structures. An advantage of placing the horizontal limb of the incision below the clavicle is that the resulting cicatrix is less liable to be chafed than it would be if this portion of the incision were placed either above or opposite that bone.

After reflecting outwards the triangular flap of skin and platysma, the posterior border of the sternomastoid is defined. In doing this the lower part of the external jugular vein is freed and divided between forceps. The outer fibres of the sternomastoid are then divided close to the clavicle, so that the muscle may be more thoroughly retracted inwards. This procedure is especially called for when the lowest trunk of the plexus is involved. The deep fascia is now divided along the upper border of the clavicle, and, along with the subjacent cushion of 
fat, is dissected upwards and backwards so as to expose the posterior belly of the omohyoid muscle which is freed and retracted, or, if necessary, resected. The transverse cervical vessels are divided between forceps as they pass outwards in front of the plexus. By retracting the sternomastoid muscle well inwards the scalenus anticus is exposed and its outer border defined and freed.

The upper trunk of the plexus is now exposed, descending upon the scalenus medius muscle. The two roots of this trunk, formed by the anterior rami of the fifth and sixth cervical nerves, may now be followed upwards and inwards behind the scalenus anticus. Note that the fifth nerve is considerably smaller than the sixth, and that it appears from behind the outer border of the scalenus anticus opposite the transverse process of the fifth cervical vertebra, which corresponds to the level of the lower border of the thyroid cartilage. Care must be taken not to mistake the trunk of the descending superficial nerve for the anterior ramus of the fifth cervical. This mistake is all the more likely to be made, seeing that faradic stimulation of the trunk of the descending superficial cervical nerve may give rise to elevation of

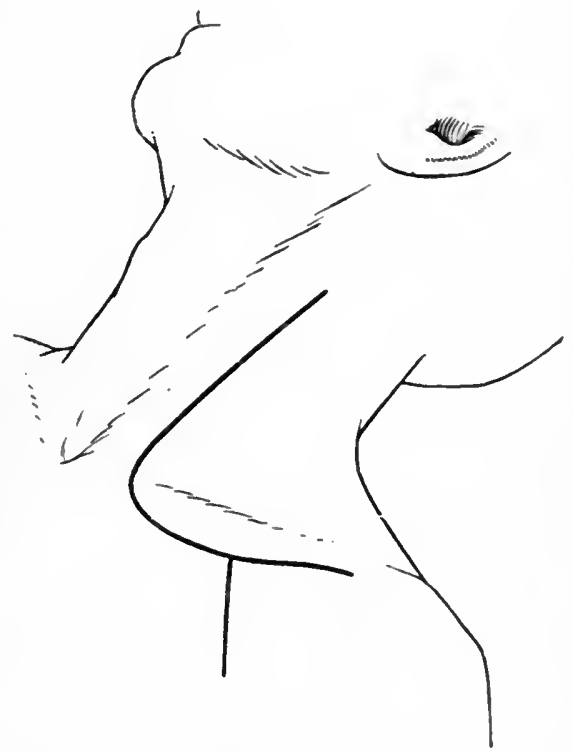

FIG. 2I.-To illustrate incision for complete exposure of the brachial plexus. the scapula, the result of contraction of the trapezius muscle. This contraction of the trapezius, which is due to an overflow stimulation of the accessory spinal nerve, might easily be mistaken for contraction of the levator anguli scapulæ and rhomboid muscles which are supplied by the fifth cervical nerve. The anterior ramus of the sixth cervical nerve descends in front of the transverse process (carotid tubercle) of the sixth vertebra, against which it is liable to be contused or even lacerated. In lesions of the upper trunk of the plexus it is not uncommon to find that this process has been fractured, in which case it will be found to be enlarged and unduly prominent. If there should be any difficulty in finding the upper trunk, the best plan is to expose the suprascapular nerve just above the clavicle and then to trace it upwards. Immediately below the origin of this nerve the upper trunk divides into its anterior and posterior divisions.

A clean bullet wound of the neck may cause an isolated lesion of this 
upper trunk, which may be either completely divided, embedded in scar tissue, or the seat of a cicatricial neuroma. If the lesion is limited to the upper trunk, its resection may be followed by end-to-end suturing of the anterior rami of the fifth and sixth cervical nerves to the two divisions of the trunk. along with the suprascapular nerve. Before, however, the distal stumps can be brought into contact with the fifth and sixth roots, the plexus must be well freed, not only in the neck but downwards behind the clavicle into the axilla. This is done by removing the adipose tissue which surrounds the plexus and is continued down along it behind the clavicle into the axilla. While this fat can be separated to a large extent by the finger, there is beneath it a fascial layer which forms a more immediate covering to the plexus and binds together its various strands. To remove this fascia a clean knife-dissection is called for. The short proximal stump, formed by the anterior rami of the fifth and sixth cervical nerves, must be freed sufficiently to allow the sutures to be introduced. For this purpose the scalenus anticus muscle must be freed and retracted inwards, so that the two roots may be followed in a proximal direction posterior to the muscle. Before this can be done satisfactorily, it is necessary to remove a quantity of fat and one or two deep cervical glands which lie along the lateral aspect of the internal jugular vein, and then to free and retract inwards the vein itself. The opportunity may now be taken to investigate the phrenic nerve as it descends upon the scalenus anticus muscle, covered by a thin layer of prevertebral fascia. This step in the operation is troublesome if the vein is bound down to the muscle by firm adhesions or scar tissue. If the nerve to the rhomboids (posterior scapular) and the nerve to the serratus magnus (long thoracic) have not been involved in the lesion, care should be taken not to injure them at this stage of the dissection. If, on the other hand, they are already involved, the chances are that the anterior rami have been damaged too near the spinal canal to admit of successful end-to-end suture. In this case the only alternative is to anastomose the distal stump into a lateral wedge removed from the anterior ramus of the seventh cervical nerve; or, if the distal stump is represented by the two divisions of the upper trunk and by the divided suprascapular nerve, one of them may be transplanted into a wedge removed from the lowest trunk (formed by the junction of the anterior rami of the eighth cervical and first thoracic nerves), the others into the seventh nerve as above described. It is a question, however, whether it is wise to injure nerve trunks which have not been involved for the sake of a problematic benefit.

When the lesion is below the fifth and sixth roots and involves the upper trunk along with its two divisions, the clavicle will generally have to be divided in order to ascertain if by freeing and pulling upwards the 
axillary portion of the plexus, it is possible to perform a resection followed by end-to-end union. If this is impossible, the operator will generally have to content himself with freeing and incising the injured trunks. If, however, these have been completely severed and it is evident that the freshened stumps could not be approximated after the bulbs have been removed, the latter should be left on and sutured to one another in the hope that by the slow-stretching method they may be removed at a subsequent operation and the freshened ends approximated. If the lesion is still more extensive, the operator will have to consider whether it is worth while doing a lateral anastomosis or a nerve-grafting operation ; in attempting the latter procedure, a graft consisting of several cables must be employed. A two- or three-ply graft of the internal cutaneous nerve of the arm or the cutaneous portion of the musculo-cutaneous nerve of the leg may be utilized. It is also worth while considering whether it might not be possible to make use of the trunk of the descending superficial cervical nerve and its proximal subdivisions.

Exposure of lowest Trunk. Next, the exposure of the lowest trunk of the plexus, namely that formed by the anterior rami of the eighth cervical and first thoracic nerves, must be described. This is done by freeing and tracing the outer border of the scalenus anticus muscle down to its insertion into the scalene tubercle of the first rib. This entails the removal still lower down of the fat and one or two of the lower carotid lymph glands, so as to expose the lowest portion of the internal jugular vein, which must be freed and retracted inwards. In working close to the angle between this vein and the subclavian vein, care must be taken not to wound either the dilated terminal portion of the external jugular vein or the transverse cervical and suprascapular veins just where they open into it. The most likely vein to be injured is the suprascapular, as it is hidden from view by the clavicle. It is important, therefore, to deliberately isolate these veins, and, having divided them between hrmostatic forceps, to displace the proximal stumps inwards along with the lower part of the internal jugular. At this stage of the dissection, care must also be taken not to injure the thoracic duct if it is the left side of the neck which is being operated on. The best way to avoid the duct is to keep the edge of the knife in contact with the structures one intends to expose ; the duct, being very elastic and surrounded by loose fatty tissue, will generally take care of itself, provided, of course, it is not involved in adhesions.

The third part of the subclavian artery is now seen and its pulsation felt as it rests on the first rib immediately external to the anterior scalene muscle. Before the artery can be fully exposed, freed, and mobilized, its sheath, formed by the prevertebral fascia, must be divided. If the vessel be now retracted downwards and inwards, the lowest trunk of the 
brachial plexus is brought into view. To expose the two roots of this trunk (8C. and I T.), the outer border of the scalenus anticus must be divided close to the scalene tubercle and the remainder of the muscle, along with the phrenic nerve and the lower part of the internal jugular vein, must be retracted downwards and inwards. The important relations of these two roots, and of their union to form the lowest trunk of the plexus, to the pleura, the subclavian artery, the first rib and the inner border of the scalenus medius muscle have already been referred to.

While extensive lesions of this trunk and its roots are generally fatal owing to their close proximity to the main vessels, there are nevertheless two types of lesions met with which are more or less amenable to operative treatment.

There is the type in which this trunk, either alone or along with the rest of the plexus, has been severely contused between the clavicle and the first rib. In this case the clavicle has usually, though not invariably, been fractured, and one of the fragments has been driven backwards against the plexus, or it is being compressed by excessive callus; or a missile may have been lodged in the root of the neck or upper part of the pleural cavity and given rise to inflammatory thickening and adhesions around and between the constituents of the plexus, especially between it, the rib, the pleura, and the subclavian artery. In these diffuse types of injury the indications are to remove all cicatricial tissue and compressing bone, and to make a clean dissection, if necessary, of the whole plexus. Wherever the sheath is much thickened to form neuromatous swellings, it should be either incised or, still better, dissected away so as to expose the nerve bundles.

In many cases in which the plexus has been contused, improvement may be brought about by non-operative methods. In others, again, operation is called for either on account of a residue of persistent paralysis or on account of severe and persistent neuralgia. For example, in a patient who suffered from severe brachial neuralgia, the writer was obliged to operate immediately after the wound above the clavicle had healed. The skiagram showed a cubical piece of shell-casing situated behind the apex of the lung in the neighbourhood of the posterior part of the first intercostal space. The neuralgia was most intense along the sensory distribution of the lowest trunk of the plexus. The adhesions involved more especially the lower end of the internal jugular vein, the lower part of the scalenus anticus muscle, the subclavian artery, the cervical pleura, the two lowest roots of the plexus, and the trunk formed by their union. In freeing the lower end of the internal jugular vein and the scalenus anticus muscle, the cervical pleura was opened into. The subclavian artery was freed and retracted downwards and inwards, after which the lowest trunk and its two roots were separated from the 
pleura and the inner border of the first rib, to both of which they were fixed by adhesions. With the subclavian artery retracted downwards and inwards, the finger was introduced into the opening made into the cervical pleura, and, after separating the adherent apex of the lung, an irregularity was felt towards the posterior part of the inner border of the first rib, this portion having been injured by the missile. As no foreign body could be felt, about $I_{\frac{1}{2}}$ in. of the first rib was resected well posterior to the subclavian artery. Before the resection, the insertion of the scalenus medius muscle was detached from the rib and the latter was freed from the parietal pleura. After removing the piece of rib, the finger could be passed behind the cervical pleura so as to separate this membrane as far back as the tubercle. When this had been done the missile could be felt behind the thickened pleura a little below the posterior part of the inner border of the first rib. In detaching the scalenus medius from the first rib, care should be taken to keep close to the bone, so as not to injure the nerve to the serratus or one of its roots, especially the lowest.

With regard to the opening which had been accidentally made into the cervical pleura, it was not possible to close it with sutures, as the edges of the opening were adherent to the internal jugular vein and to other important structures which could not be included in the sutures. In the treatment of such a complication the main point, of course, is to prevent suction of air into the pleural cavity. This is most conveniently done by carefully plugging down to the opening with a strip of iodoform gauze and then stitching the deep structures and skin as closely as possible round the end of the gauze which is brought out through the skin wound. A large dressing should be firmly fixed in position by a carefully applied bandage. When the gauze packing is removed, which should not be until the eighth or tenth day, the small opening left in the skin wound is completely closed by means of a suture. As long as the temperature remains normal, or nearly so, the slight pleuritic effusion which results from the operation may be allowed to absorb.

The type of lesion of the lowest trunk of the plexus next to be referred to is of special interest both as regards its nature and its mode of production. This lesion is characterized by the presence of a localized neuromatous thickening of the anterior ramus of the first thoracic nerve, just where it arches over the sharp inner border of the first rib to joir the anterior ramus of the eighth cervical nerve to form the lowest trunk of the plexus. The history of such a condition is that the patient had received a gunshot wound, that the bullet had traversed the upper part of the chest and the root of the neck, and that in doing so it had passed through the inlet of the thorax. The disruptive force, which radiated in all directions from the missile, caused that portion of the first thoracic nerve which joins the eighth cervical, to be severely contused by being 
driven violently against the sharp inner border of the first rib, the result being that some of the fibres were cut completely across,. while the sheath of the nerve itself remained almost intact, though thickened. A fibrous neuroma about the size and shape of a large grain of wheat developed in the substance of the lowest trunk exactly opposite the edge of the rib, that is to say, just where the first thoracic joins the eighth cervical nerve. The writer has met with two very typical examples of this condition. The symptoms are essentially the same as those which are met with in that particular type of brachial neuralgia and paresis of hand muscles which results from the gradual pressure of the inner border of the first rib upon the branch of the first thoracic as it ascends over, and is, as it were, stretched across the inner border of the first rib to join the eighth cervical nerve.

With regard to the treatment of this localized neuroma, non-operative measures should be given a fair trial, and if no improvement results after three or four months, the lowest trunk of the plexus and its two roots should be exposed in the manner just described, and the neuroma removed. Should the lesion, however, prove to be more diffuse, the involved portion of the nerve should be thoroughly freed from adhesions and its thickened sheath excised.

From the operator's point of view these casesare particularly fascinating as the track of the bullet need not necessarily, be in the immediate neighbourhood of the lesion, and therefore the dissection, although a deep and delicate one, is not complicated by the presence of scar tissue and adhesions.

Exposure of the entire Plexus. To complete the operative treatment of brachial plexus injuries it is necessary to refer to the procedure to be adopted when the lesion has involved the portion of the plexus which lies behind the clavicle along with one or all of the cords. Such lesions are liable to occur when the missile has traversed the axilla as well as the root of the neck, or when the clavicle has been fractured and the ends driven backwards against the plexus, or when the plexus is compressed between a mass of redundant callus and the first rib. There is another type of case, namely where the shoulder has been forcibly depressed, with the result that the plexus has either been contused against the first rib or ruptured in the neighbourhood of its roots. In such cases the upper two roots may be torn across, while the lower trunk of the plexus is at the same time severely contused between the clavicle and the first rib. The indication here is to make a complete exposure of the whole plexus, and, having ascertained the exact nature of the lesion, to deal with it accordingly. Unfortunately in many cases little or nothing can be done; but while this is the case, an exploratory operation should be undertaken in practically all cases, because it is often impossible to foresee from clinical evidence alone which cases are hopeless 
and which can be benefited. Not infrequently one discovers that the paralysis is due to compression, and by relieving this, rapid and marked improvement frequently takes place. The best incision for exposure of the whole plexus is the angular one already mentioned, with the addition of a third limb which extends downwards from the horizontal limb along the interval between the pectoralis major and the deltoid muscles.

The middle two-fourths of the clavicle is exposed and its muscular attachments and periosteum are freed from its middle third. It is then divided at its centre with a Gigli saw, but before doing so, it is advisable to dill the two holes through which the wire is to be threaded for the subsequent suturing of the fragments. These holes should pass obliquely through the fragments, so as to avoid irritation of the plexus by a wire on the postero-inferior aspect of the bone. If the plexus is being pressed upon by callus, the redundant portion should be removed at this stage, and in some cases it may be necessary to resect the fractured area and to remove a comminuted fragment which has been displaced backwards.

After dealing with the clavicle, the next step is the removal of the subclavius muscle, the costo-coracoid membrane and the fascia which surrounds and binds together the axillary vessels and the component parts of the plexus. To obtain sufficient access for this purpose it is advisable to retract the clavicular portion of the pectoralis muscle (which has already been separated from the clavicle) well backwards and inwards, taking care to dissect out and preserve the external anterior thoracic nerve if it has not been destroyed. The anterior fibres of the deltoid, which have also been detached from the clavicle, are retrac'ed backwards and outwards, so as to expose the insertion of the pectoralis minor into the coracoid process. The upper border of this muscle is now freed and the muscle itself either retracted well downwards, divided, or partly resected, according to the extent of the lesion. There is no object in preserving the muscle if the internal anterior thoracic nerve is destroyed. During this part of the operation, the fragments of the clavicle must be well retracted by means either of two bone hooks, or of two strips of sterile bandage. (Fig. 20, p. 8I.)

The difficulty, and it is frequently a very real one, which the surgeon is most likely to be confronted with, arises from the amount and density of the scar tissue and the manner in which it has implicated not only the nerve cords, but also the vessels, especially the axillary vein and its tributaries. The safest procedure is to begin the deep dissection by exposing the axillary vein, and the best way to do this is to follow upwards the cephalic vein to its entrance into it, and then to free the whole axillary vein so that it may be retracted well inwards. The axillary artery is next exposed and mobilized, after which the cords of the brachial plexus are dealt with. The outer cord is the most superficial, and as it descends 
it comes to lie in front of the axillary artery. Behind and lateral to this cord is the posterior division of the upper trunk which helps to form the posterior cord. By tracing the latter downwards the commencement of the musculo-spiral nerve will be reached. The inner cord and its branches are exposed by retracting the axillary artery outwards and the corresponding vein inwards. The two heads of the median are easily recognized by their relation to the axillary artery, which is embraced by them. Not infrequently the two heads of the median unite at a much lower level, while occasionally the fibres of the musculo-cutaneous nerve run along with its outer head and thence in the median itself to leave it again in the upper third of the arm. The commencement of the ulnar nerve lies deep to the inner head of the median.

The nerve lesions which may be met with in the clavicular and subclavicular regions are very varied. Moreover, they are very liable to be complicated by the presence of an aneurism, either true, false, or arteriovenous, and the operation may be rendered still more difficult by the fact that the axillary artery or vein, or both, may have been previously ligatured for the arrest of a primary or secondary hæmorrhage, and should the wound have suppurated, as not infrequently happens, the nerves may be so embedded in scar tissue that their isolation is wellnigh mpossible. No definite rules can be laid down for the treatment of such complicated cases. Patience, perseverance, and, best of all, a thorough knowledge of the anatomy of the region are the chief desiderata; indeed, in some cases the operation will have to be abandoned, and the surgeon must then console himself with the fact that he has given the patient a chance, and, while he has not been able to do him any good, he has at any rate done him no harm. Sometimes the operation, while it may have done nothing to help the paralysis, will be found to have relieved pain, and to have diminished the trophic disturbances in the hand, and to have improved the circulation of the limb.

If a resection and suture of one or more of the nerves has been performed, it will generally be necessary to divide the whole pectoralis major muscle so as to enable the distal portion of the injured nerve or nerves to be sufficiently freed for the purpose of upward traction being applied to them.

After the nerves have been dealt with and after all bleeding has been very carefully arrested, the clavicle is wired.

With regard to the muscles, it is not necessary to completely restore the attachments of the muscles or to suture the whole of the divided portion of the pectoral muscle ; indeed, if this be done and the arm kept bandaged to the side for several weeks, there is a risk that abduction would be permanently restricted.

When the dissection has been an extensive one, it is generally advis- 
able to introduce a drainage-tube to provide for dependent drainage through a stab wound, placed posteriorly.

In applying the dressing, the arm may be bandaged to the chest if the deltoid muscle is not paralysed, and the bandage should be made to grip the elbow in such a way as to produce the maximum elevation of the shoulder; if, on the other hand, the deltoid is also paralysed, the arm should be abducted at the same time as the shoulder is elevated, and to do this efficiently a plaster-of-Paris bandage is much better than a splint: in applying the plaster bandages, a Hawley table is a great advantage. If the operation has involved a dissection of the root of the neck, the head should be adducted towards the shoulder. The clbow should be kept in the flexed position, and if the posterior cord of the plexus is involved, the wrist and hand must be kept in the position described under lesions of the musculo-spiral nerve.

\section{NERVES IN MIDDLE THIRD OF AXILLA}

For descriptive purposes we may include in this dissection operations performed for lesions of any of the large terminal branches of the brachial plexus situated between the lower border of the pectoralis minor and the upper border of the latissimus dorsi. To obtain proper access to the nerves in this part of their course, the axilla must be laid freely open from the front.

The arm is abducted to a right angle, and, to cnable it to be at the same time rotated outward as much as possible, the elbow should be slightly elevated on a sandbag. The shoulder on the affected side should be brought well towards the edge of the operating table.

The incision, which is begun immediately below and internal to the coracoid process, is carricd across the pectoralis major and the anterior fold of the axilla so as to meet the arm opposite the inner border of the coraco-brachialis along which it may be continued in a distal direction as far as necessary. The cephalic vein, which is exposed in the subcutaneous tissue at the upper end of the wound, is freed and displaced laterally. The fibres of the lower two-thirds of the pectoralis major are divided transversely so as to expose the subpectoral fascia which forms the immediate roof of the axilla. When, as so often happens, the missile which has injured the nerve or nerves has traversed the axilla, this fascia is generally thickened and scarred in such a way as to bind down the pectoralis major to the subjacent biceps and coraco-brachialis muscles. After dissecting away this fascia, the inner border of the coraco-brachialis is defined and freed in an upward direction as far as the coracoid process, and downwards into the arm. In doing this, forceps are applied to one or two vessels which pass between the muscle and the axillary vessels. After these vessels have been secured, the muscle is retracted outwards, 
when the median nerve will be at once exposed lying antero-external to the axillary artery. The internal cutaneous nerve and the axillary vein are also freed and retracted inwards, the axillary artery being then fully exposed. The freeing and retracting of these structures is a perfectly simple matter distal to the lesion, and this should always be done as a preliminary measure, so that the relation of the injured nerve to the axillary artery may be clearly defined before any attempt is made to free the injured portion.

The next step in the dissection is to expose the vessels and nerve trunks above the lesion. To do this, the coraco-brachialis must be followed and freed right up to its origin from the coracoid process, and the lower border of the pectoralis minor must also be defined by dividing the fascia along it, after which the muscle can be retracted upwards and inwards. In dealing with the muscles the operator must remember the important structures which are closely related to them. For example, in freeing the upper part of the coraco-brachialis, descending branches of the acromio-thoracic vessels will be divided, and a sharp look-out must be kept for the musculo-cutaneous nerve, which enters the substance of the muscle about one inch below the tip of the coracoid, the nerve having just previously given off its motor branch to supply that muscle. If the scar tissue has involved the muscle itself, some difficulty may be experienced in isolating the musculo-cutaneous nerve, and not infrequently it will be found that the lesion has extended upwards so as to involve the outer cord of the plexus, and along with it the outer head of the median which lies in close contact with the lateral aspect of the axillary artery. The distance between the origin of the musculocutaneous nerve from the outer cord of the plexus and its entrance into the substance of the coraco-brachialis is only about an inch to an inch and a half, so that a complete lesion of the musculo-cutaneous above the muscle generally entails a resection of the lowest part of the outer cord along with the commencement of the outer head of the median and the musculo-cutaneous nerves. In order to obtain sufficient access for this purpose, it is almost always necessary to divide completely the pectoralis minor and then to free the axillary vessels higher up so that they may be more thoroughly retracted inwards. Before this can be done, the long thoracic artery is divided and the axillary fat dissected inwards. When the outer head of the median nerve is followed downwards, it will be found to join the inner a little below where the musculo-cutaneous nerve enters the coraco-brachialis muscle, that is to say, a little above the upper border of the tendon of the latissimus dorsi. One of the main difficulties in this dissection is the close relation of the nerves to the large veins. Troublesome bleeding not infrequently occurs from the venae comites of the brachial artery, which pass up into the axilla and 
generally open into the axillary vein somewhere between the lower border of the pectoralis minor and the upper border of the latissimus dorsi. It is in this situation that they are so closely related to the musculocutaneous, and especially to both heads of the median nerve. Sometimes it will be necessary to resect a portion of the axillary vein. In this case the cephalic vein becomes the most important channel for the return of blood from the upper extremity; hence care should be taken not to wound this vein in making the incision through the skin and subcutaneous tissue.

After the median nerve, along with its two heads, and the musculocutaneous nerves have been dealt with, the ulnar nerve should be examined. It is exposed by thoroughly freeing the axillary vein and retracting it either inwards or outwards, whichever is found to be the most convenient. Usually the best plan is to divide between forceps, close to their termination, the veins which proceed from the inner wall of the axilla to join the axillary vein, and then to retract the latter outwards along with the artery and the nerves already mentioned. If there is much scar tissue, the divided pectoralis major will have to be retracted well inwards, so that the fatty contents of the axilla may be freed from the vein and displaced inwards towards the chest. The intercosto-humeral and the lesser internal cutaneous nerves may be divided. If the ulnar nerve be bound down to the posterior wall of the axilla by scar tissue, it may be necessary to divide the subscapular artery close to its origin, just as is done in clearing out the axilla in cases of malignant disease of the breast. The long subscapular nerve (nerve to the latissimus dorsi), which is closely related to the subscapular vessels, must be sought for and preserved if possible.

The ulnar nerve, having been completely freed, is now followed upwards to its origin from the inner cord of the plexus a little below the pectoralis minor. If the ulnar is involved close to its origin, the inner head of the median is liable to be injured as well, and if a resection is necessary, both nerves may have to be sutured to the inner cord. The internal cutaneous nerve may be disregarded, unless there is a possibility that it may be required as a graft.

The musculo-spiral nerve is seldom involved alone at the level of this dissection. It is best exposed in the first instance below the lesion, by the same dissection as that to expose the ulnar nerve, along with which it is frequently involved. The ulnar, along with the axillary vein, is retracted well inwards, while the artery, the median and musculocutaneous nerves are retracted outwards along with the coraco-brachialis muscle. The musculo-spiral is now seen at the floor of the wound, where it rests on, and may be firmly adherent to, the subscapularis muscle. Immediately external and parallel to the musculo-spiral is the circumflex 
nerve which curves dorsally across the lower border of the subscapularis between it and the upper border of the latissimus dorsi, these two muscles forming the upper and lower boundaries respectively of the quadrilateral space through which the nerve leaves the axilla. This nerve is frequently involved along with the musculo-spiral, and, like the ulnar, it may be firmly adherent to it. In freeing it from scar tissue it may be impossible to avoid injuring the posterior circumflex vein, and sometimes even the artery may have to be divided. Another difficulty is that the nerve has such a short course that only a limited portion of it can be resected with any hope of bringing the ends into contact. In freeing the musculospiral in the proximal direction, the lowest subscapular nerve may have to be sacrificed, but the upper and middle (long) subscapulars, which supply respectively the subscapularis and latissimus dorsi muscles, can frequently be preserved. In following the musculo-spiral distally its internal cutaneous branch may be ignored, but the upper branches to the triceps which come off at, or a little above, the lower border of the latissimus dorsi should be looked for and, if possible, preserved from injury. If one or more of the other branches of the plexus has to be resected as well as the musculo-spiral, it is important to suture the latter first.

The operator must not hesitate to remove, if possible, all scar tissue from the axilla. This should be done not only to prevent its readhesion to the nerves, but with the object also of obtaining free movement at the shoulder-joint.

If the pectoralis minor muscle has not been involved in scar tissue it should be resutured, as also should the divided portion of the pectoralis major, any scar tissue it may contain having been previously dissected away. Interrupted sutures of $2 \mathrm{I}$-day iodine catgut should be used for this purpose. It is, however, a matter of little or no importance if the pectoral muscles can only be very imperfectly sutured. The deficiency in the lower part of the greater pectoral is to a large extent compensated for by hypertrophy of the clavicular fibres. This is well seen after the radical operation for cancer of the breast.

It should be remembered that the large nerves of the axilla, like the axillary vessels, are taken off the stretch by adducting the arm, so that the ends of a divided nerve which are some distance apart when the arm is abducted to a right angle may come together readily when the arm is well adducted. To suture the nerves, however, with the arm adducted, the operator must stand above the patient's shoulder and look down into the wound. Another point to bear in mind is that the arm should not be abducted for the purpose of removing the stitches, and the use of a rubber or gauze drain should be avoided. The wound should be rendered as dry as possible before closure. Drainage must be provided 
for by allowing the wound to gape a little at one or possibly two places between the sutures, all of which should be interrupted. If there is reason to suspect a. good deal of oozing, freer drainage can be got by passing several strands of catgut through a buttonhole opening made through the posterior wall of the axilla, in the interval between the subscapularis and the teres major muscles just medial to the long head of the triceps, care being taken to avoid the subscapular artery and its dorsal scapular branch. As there is sometimes a good deal of serosanguineous discharge, it is well to apply a large absorbent dressing, which should include the whole of the neck and chest as well as the whole upper extremity except the hand.

The bandage should be applied so as to maintain the arm adducted across the chest, while the elbow is kept flexed by means of a Jones double-gutter elbow splint bent to the necessary angle. A long cock-up splint of proper pattern (see p. ro7) must also be applied to the hand in cases in which the musculo-spiral nerve has been involved. When the median and ulnar nerves have been sutured, the wrist should be held palmar flexed by means of a bent single gutter splint applied to the back of the forcarm and hand.

Combined Lesions of the Nerves and Vessels of the Axilla. While the blood-vessels are sometimes found to be injured along with the nerves, it must be confessed that such combined lesions are much less frequently met with than one would expect from the very close relation of the nerves to the axillary vessels. No doubt one reason is that many soldiers who have received such injuries succumb to hæmorrhage. Nevertheless, among the patients who reach-the orthopædic centres, one sees many cases of gunshot injury to the main nerves in the axilla in which the vessels have escaped in a most remarkable manner. The artery escapes more frequently than the vein, due no doubt to the greater thickness of its walls and to its greater elasticity. In some cases one or both of the main vessels, or one of the large branches, has been ligatured to arrest the primary hremorrhage. In such cases the subsequent operation on the nerves is rendered difficult in consequence of the abundance of dense scar tissue which is often found in the neighbourhood of the injury. In other cases the nerve injury is complicated by the presence of a pulsating aneurism in the axilla, either of the true or false variety, and sometimes arterio-venous in type. In other cases again, there is a firm oval swelling which does not pulsate, and which is found to be an organized hrematoma.

In all these cases two or more of the nerves are liable to be involved, the lesion being complete in some and partial in others; in the latter case the symptoms are generally due to the nerve being stretched and spread out as it courses over the wall of the sac. Peripheral pain, along with partial paralysis and trophic lesions, are common symptoms. 
The following operation which the writer performed in a case of multiple nerve lesions of the axilla complicated with aneurism may be taken as an example of the kind of procedure to be adopted. There was a thick-walled, oval, pulsating aneurism, the size of a hen's egg, its contre being situated at the level of the lower border of the subscapularis muscle. The incision employed was the same as that above described, except that more room had to be got by the addition of a second incision at right angles to the main one which followed the line of the axillary vessels. This second incision crossed the axilla a little medial to the junction of the arm and chest. After the flaps had been reflected, the sternal fibres of the pectoralis major and the subpectoral fascia were divided transversely until the pectoralis minor was exposed. The axillary vein and median nerve were then freed below the aneurism and retracted outwards along with the coraco-brachialis muscle. The ulnar was retracted inwards. The lower part of the axillary artery was then exposed and traced upwards to the aneurism. The next step consisted in freeing the pectoralis minor, which was retracted well upwards. The axillary fat was then removed from the upper part of the axilla, the intercosto-humeral nerve divided, and forceps applied to one or two unimportant branches of the axillary vessels. This enabled the axillary vein to be freed and separated from the axillary artery above the level of the lesion. The artery was then isolated and clamped immediately above the aneurism, and a second pair of forceps was applied to the vessel immediately below the aneurism. The securing of the artery as close above and below the aneurism as possible is an essential step in the operation and one which should be carried out at as early a stage as possible ; indeed, it is the primary object of what may be regarded as the first stage of the operation. The artery having been controlled, the median nerve, along with its two heads, and the ulnar nerve were then carefully dissected off the anterior wall of the sac, while the musculospiral was exposed on the proximal side of it. When freed, the two former nerves were found to be so attenuated and flattened that little else than their sheaths remained.

The anterior wall of the sac was now laid freely open and some old laminated clot rapidly removed, when free, but not alarming, bleeding took place from two openings in the wall of the sac. The bleeding was arrested by clamping the portions of the sac containing these openings with strong artery forceps. The remainder of the clot was then removed from the sac, partly with the finger and partly with a sharp spoon. The sac was found to extend for a considerable distance upwards behind the vessels, between them and the subscapularis muscle. After the anterior wall of the sac had been cut away, the axillary artery was ligatured where it was clamped immediately above and below the aneurism. The 
posterior wall of the sac was so adherent to the deeper structures that only a portion of it could be removed; the remainder was firmly adherent to the musculo-spiral nerve ; indeed, the latter actually formed part of the wall of the sac. The circumflex nerve was not identified. The lowest subscapular nerve was preserved, but the middle (long) subscapular was involved in the sac. The subscapular artery was ligatured, and the posterior circumflex was certainly one of the vessels opening into the sac.

The final steps of the operation consisted in the resection of $I_{\frac{1}{2}}$ in. of the median and ulnar nerves and suture. Fortunately the musculospiral gave a fairly good response to the faradic current so that it was not necessary to interfere with it. The treatment of the wound was the same as that described in the previous operation. The ulnar nerve has now completely recovered, and the median nearly so.

\section{NERVES IN THE LOWER THIRD OF THE AXILLA AND PROXIMAL HALF OF THE UPPER ARM}

All the terminal branches of the three cords of the brachial plexus are so closely related to the main blood-vessels of the axilla and upper half of the arm that for descriptive purposes and to avoid repetition they may with advantage be considered together, especially as the lesion may involve two, or even more, of the nerves. The chief nerves to be considered in this region are the median, the ulnar, and the musculo-spiral. All these nerves are best exposed by the normal incision employed for exposure of the lower half of the axillary artery and the upper half of the brachial. The incision begins at the apex of the axilla opposite the medial border of the coraco-brachialis, which corresponds to the junction of the anterior and middle thirds of the outer wall of the axilla. From this point it is carried down along the inner border of the muscle as far as may be necessary. Should it be found necessary later to trace the nerve still higher in the axilla, the incision may be best extended upwards by carrying it along the free border of pectoralis major. At the upper part of the incision the axillary fascia is exposed and below this the deep fascial envelope of the arm. Through this fascia the internal cutaneous nerve can usually be seen coursing along the anterior aspect of the basilic vein. The deep fascia should be incised along the inner border of coracobrachialis, instead of directly over the neuro-vascular bundle. The internal cutaneous and basilic vein are both freed and retracted backwards. The vein is liable to be wounded accidentally if the incision penetrates too deeply just posterior to the nerve. The inner border of the coraco-brachialis muscle is then freed and retracted forwards and outwards. In doing this a few muscular branches of the brachial artery, along with their companion veins, are divided and ligatured. The median nerve is now exposed lying in contact with the anterior aspect of the 
brachial artery. When it has been freed in an upward direction, its two heads will generally be found to join and embrace the artery about the level of the upper border of the latissimus dorsi, which may be regarded as the upper limit of the dissection we are now considering.

The next step in the dissection is the exposure of the ulnar and musculo-spiral nerves. To lay bare the ulnar, the basilic vein may be retracted either forwards or backwards, when the nerve will be found lying immediately posterior to the axillary and brachial arteries. Like the median, it gives off no branches either in the axilla or the upper arm, so that it is easily and rapidly freed in both directions. (Fig. 22.)

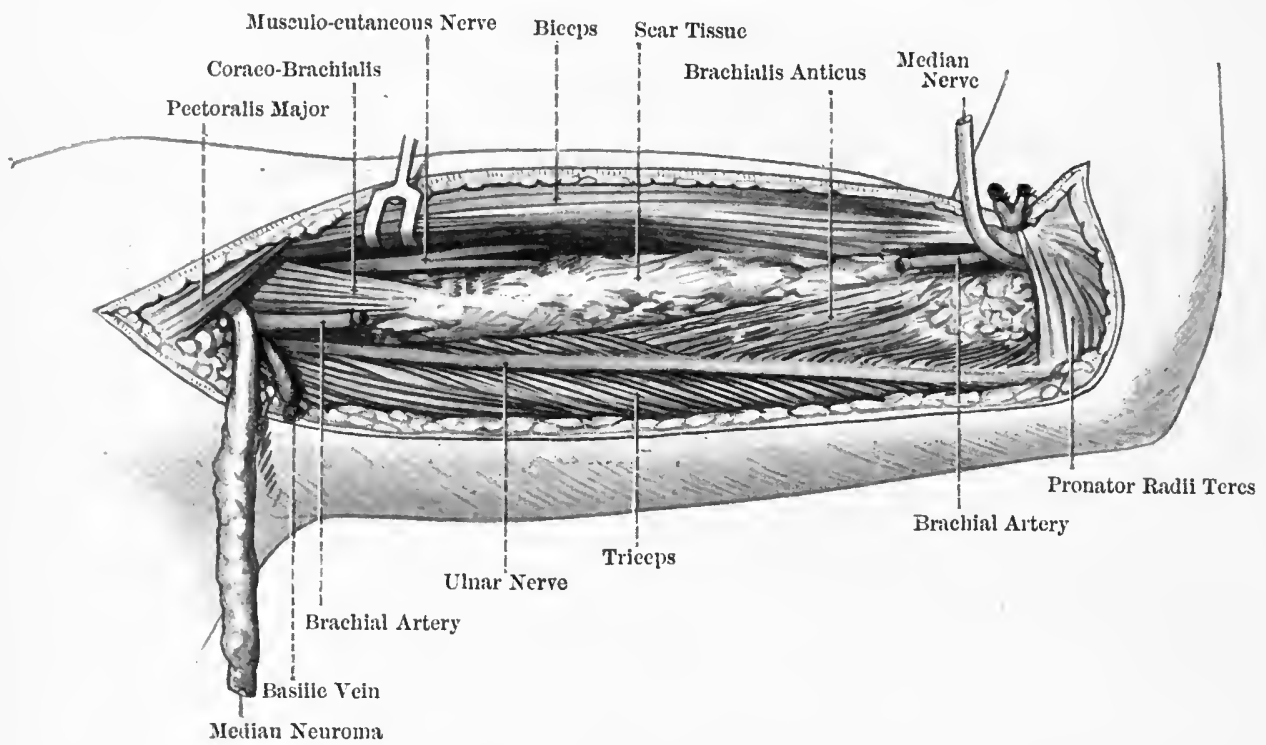

FIG. 22.-Operation for lesion of median and ulnar nerves in the middle of the arm. Median involved in mass of scar tissue at insertion of coraco-brachialis and artery thrombosed in middle third of arm.

If it be a lesion of the musculo-spiral with which we have to deal, two methods of approach are available. In the one, the main vessels, along with the internal cutaneous, the median, and the ulnar nerves, are retracted inwards and backwards; while in the other, these structures are retracted in the opposite direction, namely, forwards and outwards. The writer desires particularly to draw attention to the great advantage of the former over the latter method. It will be remembered that the musculo-spiral nerve takes an outward direction as it courses distally behind the humerus to enter the musculo-spiral groove. If the lesion be situated either in the lower part of the axilla or in the proximal half of the arm, the incision should be precisely the same as for exposure of the 
median nerve. The musculo-spiral is first exposed above the lesion, that is to say, where it lies behind the axillary artery upon the tendon of the latissimus dorsi, close to its insertion. Here it is readily exposed by retracting the coraco-brachialis muscle and musculo-cutaneous nerve well outwards, while the main vessels, along with the nerves already mentioned, are all retracted backwards and inwards. To follow the nerve as far as

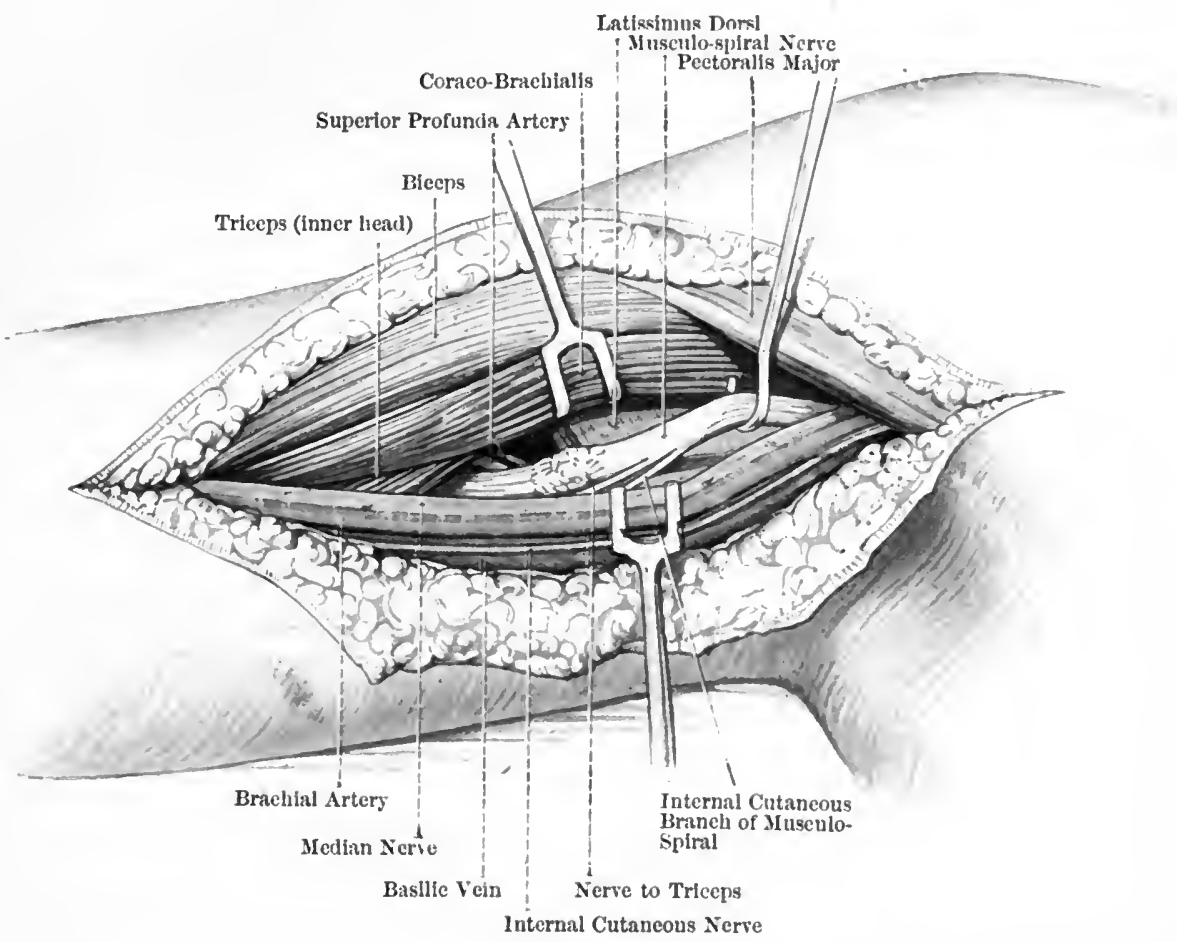

FIG. 23.-Operation for a neuroma of musculo-spiral (N. radialis) in upper third of arm, immediately below the branches to the triceps. Note that the median nerve and main vessels have been retracted inwards and backwards.

the musculo-spiral groove, the dissection is continued downwards deeply between the brachial artery and the coraco-brachialis muscle. (Figs. 23 and 24.) The artery, along with its venæe comites and the basilic vein, and also the median nerve are freed in a distal direction as far as the insertion of the coraco-brachialis; if they are now retracted well inwards and back. wards, the superior profunda vessels will come into view so that they may be divided between forceps and ligatured, a step which greatly facilitates the following of the nerve into the upper part of the musculo-spiral groove. In a certain proportion of cases (one in five, according to Quain) the musculo-cutaneous nerve leaves the outer head of the median at 
a much lower level than usual, even as low as the insertion of coracobrachialis. This arrangement makes more difficult the exposure of the musculo-spiral by inward retraction of the brachial vessels and nerves.

The branches which arise from the musculo-spiral in this dissection are the internal cutaneous, the ulnar collateral to the inner head of the triceps, and branches to its long and outer heads. All these branches come off close together at the level of, or a little below, the lower border of the latissimus dorsi ; by careful dissection, they can generally be preserved intact when the lesion is placed a little above the musculo-spiral groove, and, if necessary, they can be traced up from the main nerve as a separate

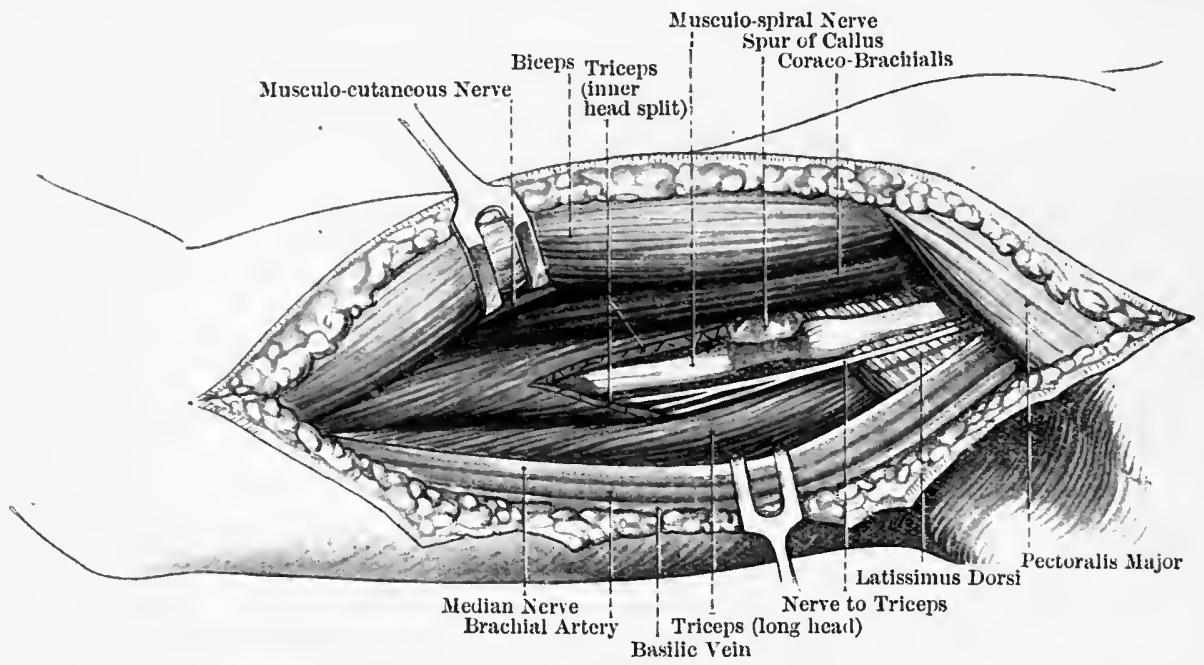

FIG. 24.-Operation to free the musculo-spiral (N. radialis) from a spur of callus. Note the bulbous swelling immediately above the compressed portion of the nerve. Lesion exposed by retracting the median nerve and main vessels backwards and inwards. Medial head of triceps divided and radial nerve traced distally along the musculo-spiral groove. Branches to triceps freed from the injured portion of the nerve.

bundle high up into the axilla. The nerve can be followed for a considerable distance along the musculo-spiral groove without altering the position of the limb and, what is more important, without dividing the outer head of the triceps. All that is necessary is to divide the upper fibres of the inner head of the triceps close to the humerus. The superior profunda artery has already been secured, but forceps will again have to be applied to the companion vein at a lower level. In this way the musculo-spiral may be exposed and freed from the upper border of the latissimus dorsi down to the middle of the groove, and this exposure will admit of an end-to-end suture after resection of $I_{2} \frac{1}{2}$ in. of the nerve, provided the arm is adducted. 


\section{MUSCULO-SPIRAL NERVE IN THE LOWER HALF OF THE ARM AND ITS TRANSPOSITION IN DIFFICULT CASES}

This operation involves the dissection to expose the nerve from the lower end of the musculo-spiral groove to its bifurcation, which takes place opposite the external condyle of the humerus under cover of the supinator longus muscle. In the upper third of the dissection, the nerve lies immediately behind the external intermuscular septum; in the middle third it lies in the substance of the septum; while in the lowest third it is separated from the septum by the supinator longus and extensor carpi radialis longior muscles. The incision to expose the nerve should, therefore, cross the septum very obliquely, its upper extremity being placed a little behind the insertion of the deltoid and its lower extremity midway between the biceps tendon and the external epicondyle of the humerus. The median cephalic vein is divided between forceps at the lower end of the incision, while at its middle the proximal and distal dorsal cutaneous branches of the musculo-spiral are exposed. The former, which courses downwards and forwards in front of the septum, is the smaller, and may be ignored; the latter, which takes a dorsal direction, is more important as it serves as a useful guide to the musculospiral. By tracing this cutaneous branch in the proximal direction it will be found to pierce the external intermuscular septum, and by following it upwards in the substance of the septum its origin from the musculospiral nerve will be reached. (Fig. 25.)

Another method of finding the nerve is to expose the anterior border of the supinator longus muscle at the lower end of the incision, and then to free it in an upward direction so that it may be well retracted, after which the nerve will be found close to its bifurcation, deep down in the interval between this muscle and the brachialis anticus. During this step of the dissection, branches of the radial recurrent vessels must be secured and ligatured. The opcrator is warned against searching for the nerve in the first instance opposite the middle of the wound, because in this situation there is practically no guide to the interval between the supinator longus and the brachialis anticus muscles, and there is a distinct likelihood that the nerve will be missed owing to the dissection being carried into the substance of the brachialis anticus instead of between it and the supinator longus. The best plan, therefore, is to begin by exposing the nerve either at the upper or at the lower part of the wound (Fig. 25.)

The main trouble in the operation is, however, generally due to the dense scar tissue which often surrounds the nerve and binds it firmly down to the site of an old fracture of the humerus. In dissecting away 
this scar tissue a good deal of inconvenience may be caused from the bleeding of the superior profunda vessels which are very closely related to the musculo-spiral nerve.

After resecting the injured portion of the nerve, it will generally be necessary to prolong the incision into the antecubital fossa in order completely to free the nerve down to its bifurcation. During this step of the operation it will generally be necessary to sacrifice the nerve to the supinator longus, and sometimes also that to the extensor carpi radialis longior. Every attempt should, however, be made to preserve the branch to the extensor carpi radialis brevior which arises from the

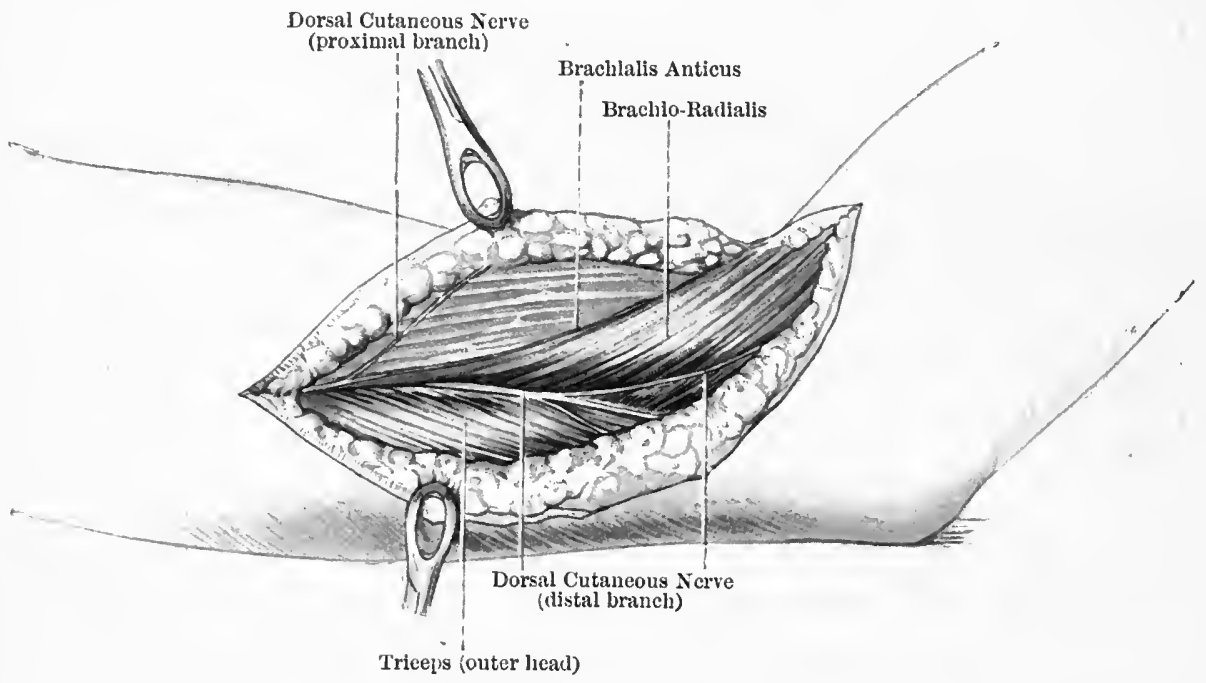

FIG. 25.-First stage of operation to expose the musculo-spiral (N. radialis) in lower third of arm. At the upper part of the dissection the superior and inferior dorsal cutaneous branches of the musculo-spiral are seen emerging from the external intermuscular septum. The interval between the brachialis anticus and the supinator longus (brachio-radialis) muscle is defined by defining the antero-medial border of the latter muscle at the bend of the elbow and then tracing it upwards.

commencement of the posterior interosseous. The small branch to the brachialis anticus is of no importance, as this muscle derives its chief nerve supply from the musculo-cutaneous nerve.

If there is troublesome oozing from cicatricial tissue, the wound will require to be drained.

Tension on the sutured nerve is best avoided by maintaining the forearm in the flexed and pronated position.

Next, let us suppose we have to deal with an extensive lesion of the nerve as it occupies the musculo-spiral groove. The usual procedure is to make a long oblique incision across the back of the arm in the line of 
the nerve, which is then exposed by dividing the outer head of the triceps. The disadvantages of this method are: (I) the division of the muscle, and (2) the inability to bring the divided ends of the nerve into apposition when, as so often happens, $3 \mathrm{in}$. or so have to be resected. It is in such cases that the transposition method proves so valuable. Suppose, for example, that the musculo-spiral is extensively involved at the upper end of the groove. The nerve is first exposed on the inner side of the arm in its upper half between the lesion and the axilla, care being taken not to injure those of its motor branches which have already been referred to as coming off opposite the insertion of the latissimus dorsi. The lesion itself is then followed into the upper part of the musculospiral groove. The steps of this operation have been already fully described (see p. Ior).

The nerve is now exposed at the junction of the middle and lowest thirds of the arm through a separate incision in the manner described above, and the lesion is then followed upwards into the lower part of the groove. (Figs. 25, 26, and 27.) The two dissections can be made to meet without actually joining the skin incisions and, what is more important, without cutting across the outer head of the triceps. The whole extent of the lesion having been freed from its adhesions to the triceps and humerus, the nerve is divided above and below the destroyed portion. An oblique tunnel is then made in an upward direction beneath the brachialis anticus muscle, between it and the front of the humerus, and forceps are passed along it in the opposite direction, so as to grasp the lower stump of the nerve and pull it upwards through the tunnel until it emerges above the upper end of the groove where it is sutured to the upper stump. If, on the other hand, the lesion is situated at the lower end of the musculospiral groove, the same dissections are made, the only difference being that the upper stump is pulled from above downwards through the tunnel and united to the lower stump at the lower end of the groove. It will be observed that the motor branches of the musculo-spiral come off for the most part above and below the resected portion of the nerve; it is only the dorsal cutaneous branches, an unimportant branch to the brachialis anticus, and possibly, the branch to the supinator longus that have to be sacrificed. In applying the dressing, the elbow should be maintained in the position of full flexion with the forearm pronated.

The war has taught us that resection of the musculo-spiral nerve followed by end-to-end sutures gives results superior to those which follow resection and suture of any other nerve, the explanation no doubt being that the musculo-spiral is almost entirely a motor nerve, and that we are accustomed to measure the results of nerve-suture operations according to the degree of restoration of the motor, rather than of the 


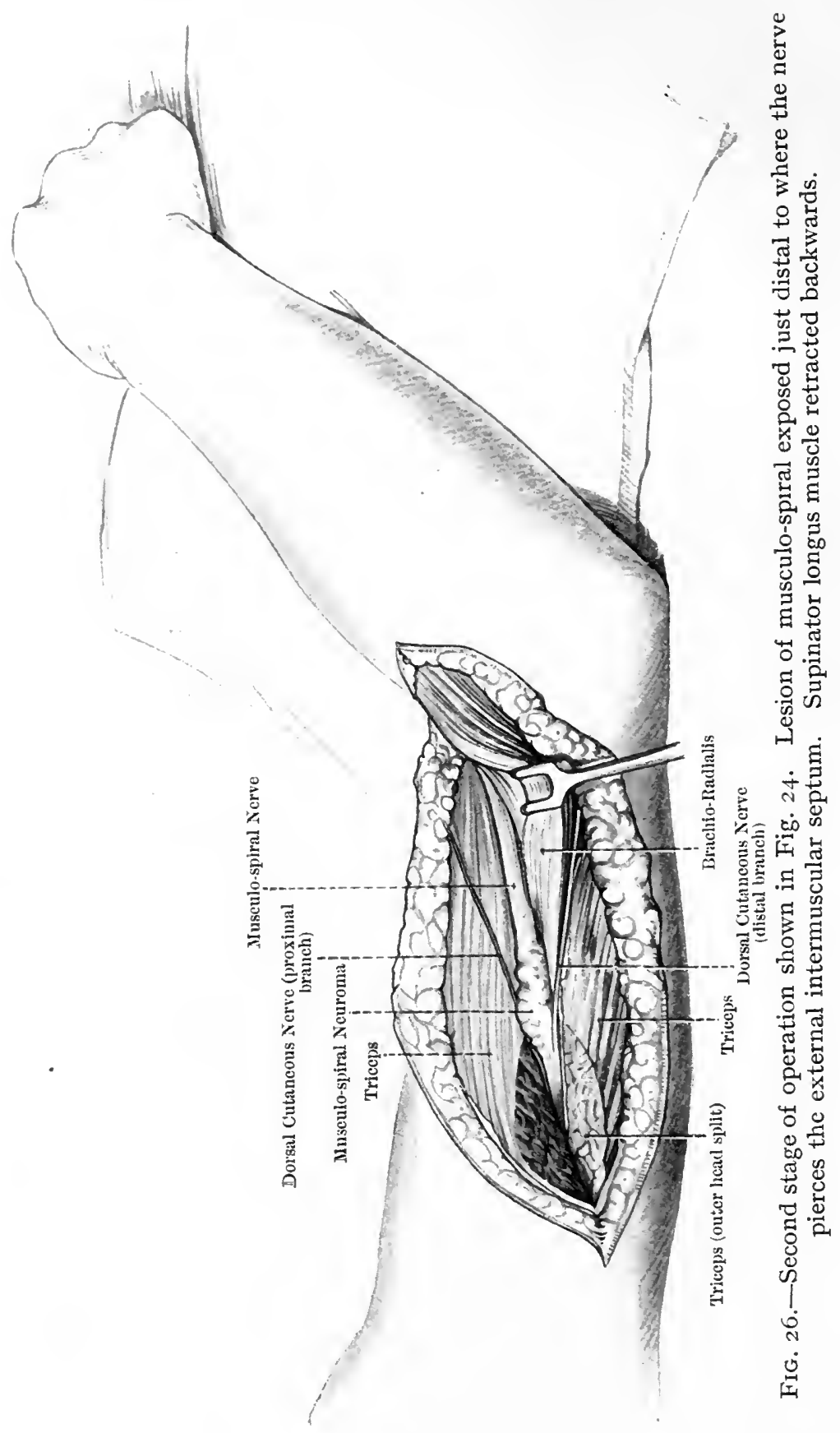


sensory and trophic, functions. Seeing then that the results of suture of the musculo-spiral are so good, it is the duty of the operator to take advantage of any step in the technique of the operation which will enable the divided ends to be brought into apposition without tension after the resection of as long a segment of the nerve as possible. Captain M. S. Danforth, one of my senior assistants at the Orthopædic Centre of the Edinburgh War Hospital, found that by this method of transposition of the nerve from the back to the front of the humerus, he was able to

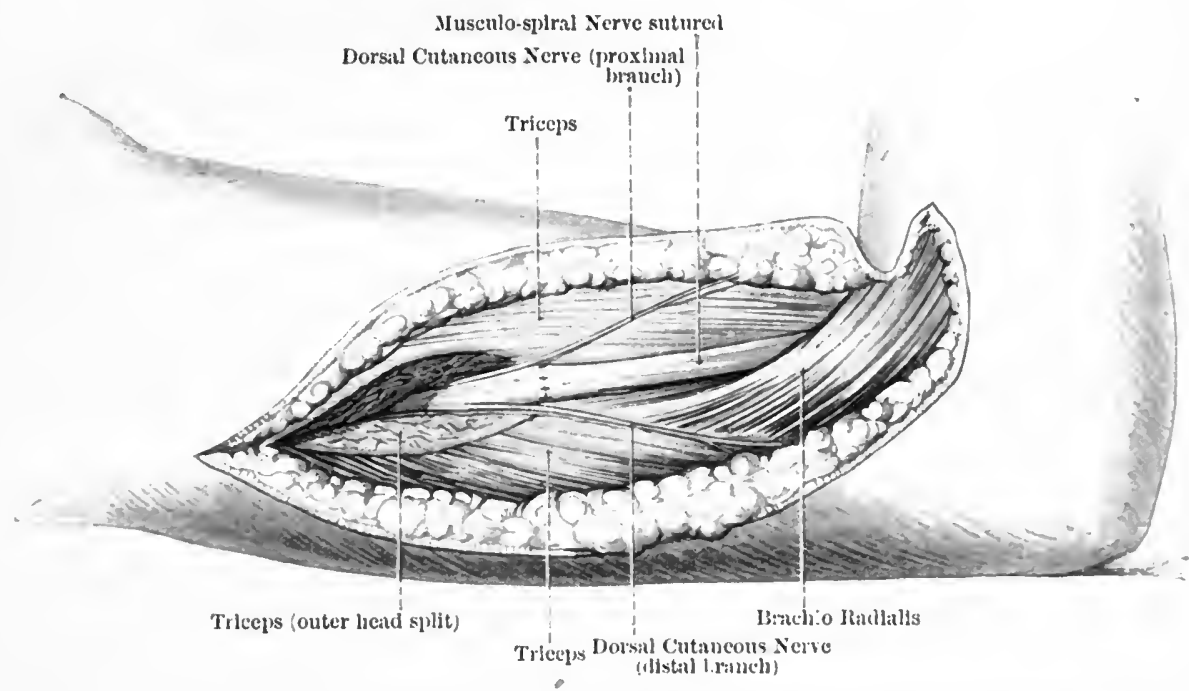

FiG. 27.-Third stage of operation shown in Figs. 24 and 25. Neuroma resected and stumps sutured. Forearm flexed and pronated to relax the nerve.

gain an inch; that is to say, he was able to bring into apposition stumps which would have remained an inch apart had he not transposed the nerve from the back to the front of the humerus.

The writer has himself carried out Captain Danforth's method and can strongly recommend it whenever the operator finds difficulty in bringing the ends together after an extensive resection of the nerve in or near the musculo-spiral groove. The gain is due only partly to the fact that by transposing the nerve to the front of the humerus it is made to take a straighter and more direct course; it is due still more to the fact that, when the nerve is brought entirely in front of the humerus, it is capable of much greater relaxation when the elbow is flexed than would be the case if it were left in its natural position behind the humerus.

With regard to the position of the limb after operation, the arm is adducted and the elbow flexed in the manner already described on p. 97. 
A special splint is required to maintain the wrist and hand in the proper position, namely, with the wrist dorsiflexed to an angle of $45^{\circ}$ and the metacarpo-phalangeal and interphalangeal joints in a position a little short of free extension. The thumb should be kept extended in the position it takes up when grasping a tumbler.

If there is every reason to believe that the lesion of the musculospiral is so extensive that even by transposing it the divided ends cannot be brought together, or if an exploratory incision shows this to be the case, the drop-wrist can be dealt with most satisfactorily by tendon transplantation. Indeed, it is safe to say that, of all the operations which the war has added to the orthopædic list, none have given more gratifying results than the operation of tendon transplantation in cases of musculospiral paralysis in which nerve suture is out of the question.

The splinting of the wrist and hand and the after-treatment are very essential, and for the necessary details the reader is referred to the section dealing with tendon transplantations (pp. $5_{54}$ and $\mathrm{I}_{56}$ ).

\section{POSTERIOR INTEROSSEOUS NERVE}

Lesions of the posterior interosseous nerve and its branches are seldom amenable to direct surgical treatment, because no sooner has the nerve trunk traversed the supinator brevis muscle than it proceeds to break up into a leash of small branches, some of which enter the deep surface of the extensor digitorum communis muscle, while others descend beneath that muscle to supply the extensor carpi ulnaris and the special extensors of the thumb and index finger.

Operations on the back of the forearm are generally performed for the purpose of removing scar tissue and repairing muscles and tendons, or for tendon transplantations, and in these operations the surgeon is concerned more with avoiding than with attacking the posterior interosseous nerve and its branches. It occasionally happens, however, that the posterior interosseous nerve is injured between its origin from the musculo-spiral and its exit from the substance of the supinator brevis muscle. The best access to the nerve is got by freely mobilizing the two radial extensors of the wrist. This is done by splitting the intermuscular septum first between these muscles and the supinator longus and then between them and the extensor digitorum communis ; then, by separating the extensor carpi radialis longior from the supinator longus right up to the external supracondylar region of the humerus and retracting the latter muscle well forwards, the bifurcation of the musculo-spiral nerve is reached. If the radial extensors of the wrist be now well freed down the forearm and retracted to the radial side, while the extensor digitorum communis is retracted towards the ulnar side of the forear $m$ 
a space is opened up at the bottom of which the oblique fibres of the supinator brevis muscle are exposed. By dividing the fibres of this muscle in the line of the nerve, the latter is fully exposed, and if only a limited portion of it has been destroyed an end-to-end suture can be effected by fully flexing as well as fully pronating the forearm. If the stumps cannot be approximated, a portion of the radial nerve should be used to bridge the interval.

In splinting the limb at the close of the operation flexion and pronation of the forearm may be maintained by means of a Jones's double-gutter elbow splint, the wrist and fingers being dorsiflexed on a long cock-up splint.

\section{ULNAR NERVE}

In Lower Two-thirds of Forearm. Here the ulnar nerve gives off no motor branches, and of the two sensory branches which come into the field of operation, one, namely the palmar cutaneous branch, may be disregarded, while the other, the dorsal digital branch, is more important. It should be preserved if possible, because blisters and ulcers are peculiarly liable to occur as a result of anæsthesia of the skin over the dorsal aspect of the little finger.

In dealing, however, with extensive lesions of the ulnar, proximal to the dorsal digital branch, the latter may prevent the lower stump being pulled far enough upwards to meet the upper stump. When this is the case, the operator should not hesitate to divide it, if by so doing the stumps can be approximated, it being taken for granted, of course, that the branch has previously been extensively freed in both directions.

To expose the ulnar in the lower two-thirds of the forearm, an incision is made along the lower two-thirds of a line extending from the posterior aspect of the internal epicondyle to the outer border of the pisiform bone. The superficial veins having been secured, the deep fascia is split in the line of incision. The nerve should be exposed in the first instance in the lower third of the forearm. The well-defined edge of the tendinous portion of the flexor carpi ulnaris muscle is the best guide to the nerve. (Fig. 28.) After this tendon has been freed and retracted well inwards and somewhat backwards, the nerve will be scen to lie immediately dorsal, rather than internal, to the ulnar artery and its venæ comites. Note that the nerve is in close contact with these vessels and that all these structures are covered by a thin layer of fascia, which binds them down to the inner border of the flexor digitorum profundus muscle. The dorsal digital branch above referred to arises from the ulnar about the junction of the middle and lower third of the forearm. If necessary, it may be 
as nearly as possible by means of traction sutures or shortening the course of the nerve by transposing it to the front of the elbow. By the latter procedure, not only is the nerve made to take a straight and therefore a shorter course, but by placing it in front of the limb we are able to take full advantage of flexion of the elbow, which serves to shorten its course still more. This is a procedure which the writer has invariably chosen, in the first place because the nerve can generally be transposed without sacrificing its motor branches to the flexor carpi ulnaris and the inner half of the flexor profundus muscles, and, secondly, because we have now had a sufficient number of recoveries after adopting this method to know that the extensive freeing of the nerve, combined with its transposition, does not interfere with its regeneration. In the rare cases where, in order to get the stumps together, it is necessary to make the transplantation still more complete, the branches above mentioned have to be divided (Fig. 30); the sacrifice is more than justified, because we now know that in the long run the patient will generally regain sensation and the use of the ulnar intrinsic muscles of the hand, which will more than compensate him for the paralysis of the flexor carpi ulnaris and of the profundus tendons to the ring and little fingers. Moreover, this paralysis can to a large extent be circumvented by dividing the two inner tendons of the flexor profundus muscle and suturing them to the two outer tendons, which receive their supply from the median nerve.

If the lesion of the ulnar nerve should happen to be in the upper part of the forearm, its motor branches may have already been destroyed, in which case there need be no hesitation about doing a complete transposition. Should it happen that the patient's elbow is also ankylosed at an obtuse angle, it may not be possible to approximate the stumps in consequence of the inability to flex the elbow. Unfortunately, it is in just such a case that little can be hoped for by applying traction sutures to the bulbs, as the only means by which the nerve can be lengthened is by gradually extending and then dorsiflexing the wrist. It is, therefore, in a case of this kind that we may have to resort to nerve-grafting, to a flap operation, or to shortening of the humerus. If the case were a suitable one it might be worth while considering the advisability of doing an arthroplasty of the elbow before attacking the nerve.

In describing the operation of transposition and suture of the ulnar nerve we cannot do better than take as an example a case in which the nerve has been extensively injured in the lower two-thirds of the forearm, and in which it has been necessary to resect more than $3 \mathrm{in}$. The operation will be a continuation of that which has just been described. The incision, which has been confined to the lower two-thirds of the forearm, is prolonged upwards, a little in front of the internal condyle, and is con- 


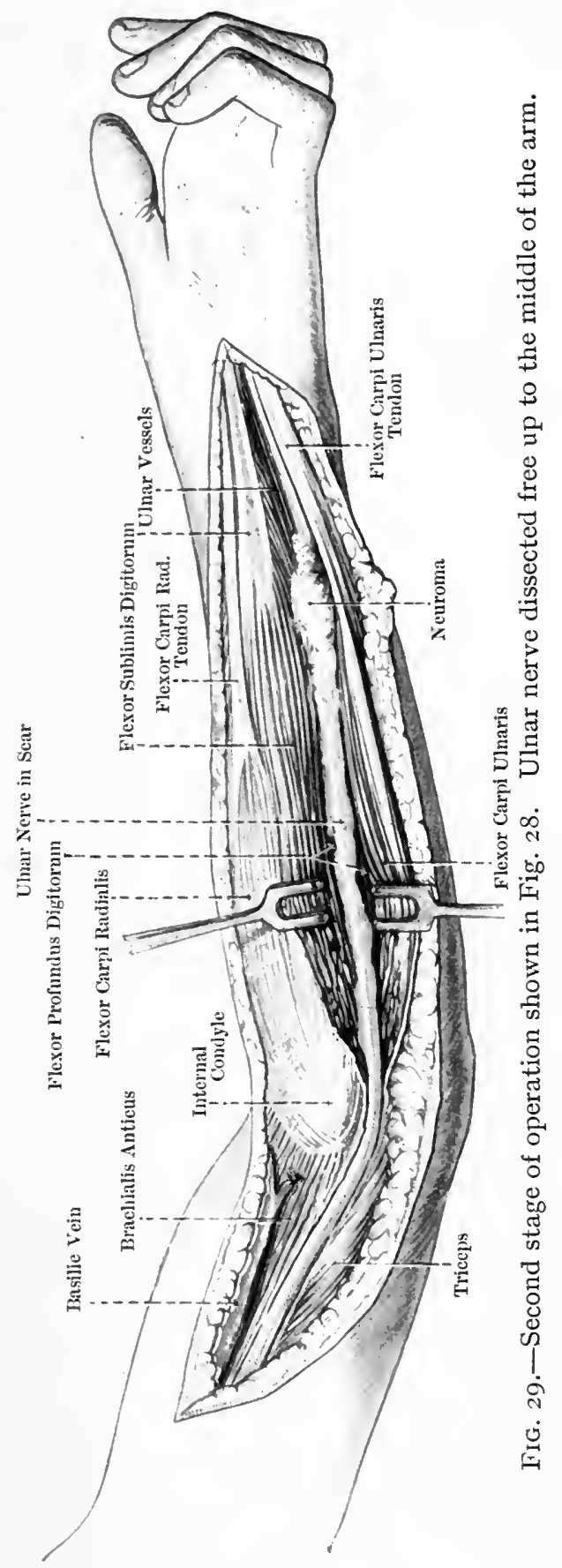

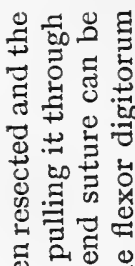

ฮี

क

胥先

흘 코

过茟

崩

嵌节导艺

후 $\overrightarrow{7}$

물

क्ष

总寻焉

䒠虽

है

EF $\exists$

的瓷

은

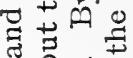

क人 인요

ज.

记热 욜

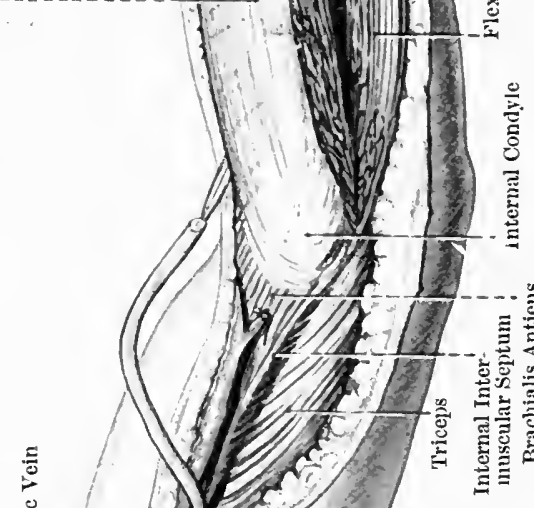

तै 过

总氙㟧

का क्ष तै क

포요코융

可记

잉

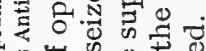

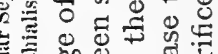

施

on

단듀

뭅요

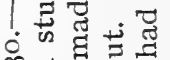

됴 है च

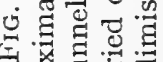

的离司泀 
tinued up the inner aspect of the arm a little behind the medial border of the biceps and coraco-brachialis muscles. Both edges of the wound are undermined in the region of the elbow. The anterior flap is reflected forwards so as to expose the outer border of the pronator teres muscle, and in doing this the median basilic vein is secured by two forceps and divided between them. The internal cutaneous nerve may generally be preserved. The posterior flap is reflected back beyond the internal condyle, and, higher up, behind the internal intermuscular septum. The ulnar nerve is now exposed, as it lies immediately behind the lower part of the septum, where it is sometimes so sunk in the substance of the triceps as to be almost hidden from view. It is in this situation that the inferior profunda artery will be encountered; here it can generally be freed along with the nerve, but, behind the internal condyle, where it anastomoses with the ulnar recurrent vessels, it will have to be divided, and it must be secured with forceps, as the bleeding is troublesome. The nerve is now freed from the back of the internal epicondyle. Here it is covered by a thick fascia, which is often so closely adherent to the nerve that care must be taken not to injure it. The nerve is next followed into the forearm by splitting the flexor carpi ulnaris muscle in the interval between its humeral and ulnar origins, and the dissection is continued downwards through the substance of the muscle until the upper limit of the previous dissection is reached. It is at this stage of the operation that the branches which are given off by the ulnar nerve to the forearm muscles are carefully exposed and freed, as far as possible, in both directions. Each head of the flexor carpi ulnaris receives a separate branch, each of which arises from the corresponding aspect of the nerve about an inch below the internal condyle. The inner portion of the flexor digitorum profundus receives two or three branches which arise about an inch lower down. If the gap in the ulnar nerve is a large one, it will be necessary to follow it through the internal intermuscular septum and to free it well up towards the axilla. This is done with the object, firstly, of straightening out the slight but distinct angle which the nerve makes as it enters the septum, and, secondly, so that full advantage may be taken of applying traction to the nerve before it is sutured. In tracing the nerve through the septum care must be taken not to injure the small ulnar collateral branch of the musculo-spiral which courses downwards immediately in front of it to supply the inner head of the triceps.

The proximal segment of the ulnar nerve having been freed from the lower part of the forearm to the upper third of the arm, it is now brought in front of the elbow as far towards its centre as the dissected-out motor branches will admit. The nerve must not be allowed to remain superficial to the internal condylar group of muscles, partly because it would be too much exposed to irritation, so that a painful neuroma would 
be liable to form, and partly because the maximum relaxation of the nerve would not be obtained if it were left to arch over the convexity of the muscles. These muscles, namely the pronator teres, the flexor carpi radialis, the palmaris longus, and the subjacent fibres of the flexor digitorum sublimis, are divided opposite and in the direction of the transposed nerve, and the latter is then sunk into the muscular trough, the floor of which is formed by the upper fibres of the flexor digitorum profundus muscle. The divided muscles are then sutured over the nerve. (Fig. 3r.)

It will be observed that by adducting the upper arm and fully flexing

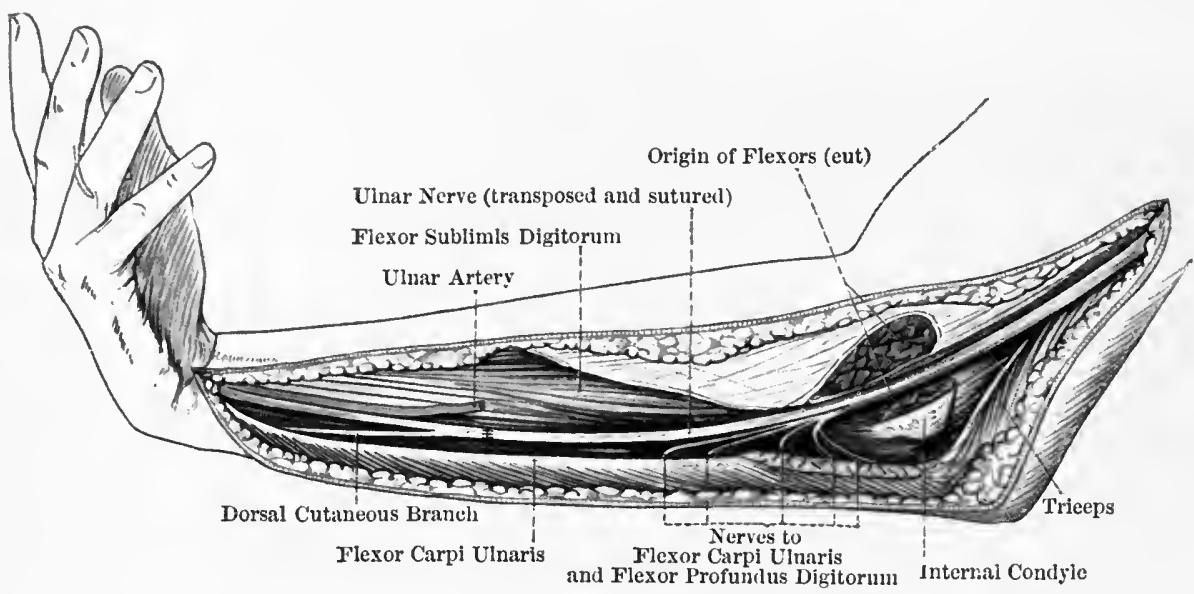

FIG. 3I. End-to-end suture of ulnar nerve after resection of a lesion involving it in the middle third of the forearm. The nerve has been freed from the wrist to the junction of the lower and middle thirds of the arm. The branches have been freed so as to allow of the transposition of the trunk of the nerve to the front of the internal condyle, where it has been sunk into a trough made in the flexor muscles, which are then sutured over the nerve. The elbow and wrist maintained flexed to prevent tension on the sutures.

the elbow and ulnar-flexing the wrist, along with traction on the two segments of the nerve (before the bulbs have been removed), the freshened stumps may be approximate even after 4 or 5 in. of the nerve has been resected. Reference to Fig. 32 will show that the proximal segment may be pulled downwards to such an extent that the motor branches of the ulnar may be made to take a recurrent direction as they pass from their origins to enter the muscles.

If these motor branches have already been destroyed, or if it is necessary to sacrifice them in order that the ulnar nerve may be made to take a straighter course, the muscles above-mentioned need not be divided. Instead of this the proximal segment of the nerve is pulled from above 
downwards through the tunnel made beneath the muscles, as shown in Fig. 30. The tunnel is made partly with a blunt instrument, such as a Kocher's dissector, and is then widened, either by forceps or by the index finger. In making the tunnel, care must be taken to see that it runs beneath and not through the fibres of the flexor digitorum sublimis, and the operator must take care also not to injure the ulnar artery.

After the nerve has been pulled downwards through the tunnel, it is important to see that it is neither kinked nor tacked down in any

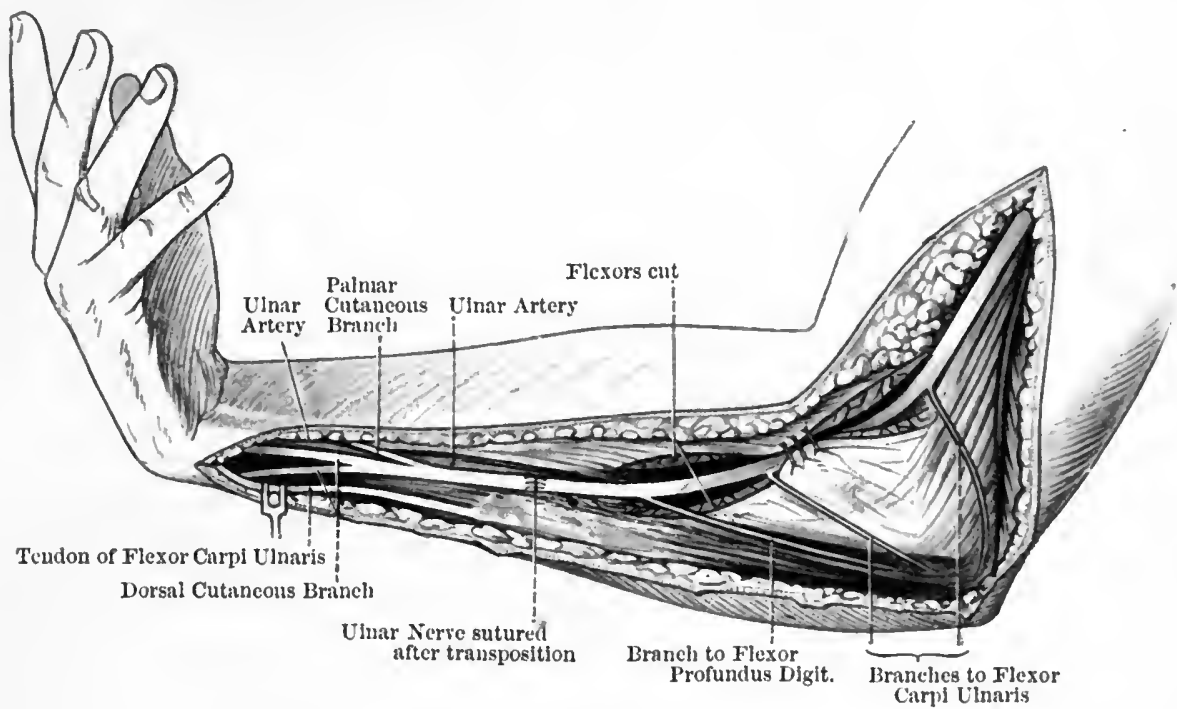

FIG. 32.-Operation similar to that shown in Fig. 30 except that more of the ulnar nerve ( 4 in.) had to be resected. Note the recurrent course taken by the motor branches after the proximal stump has been pulled down and sutured to the distal stump. Flexor carpi ulnaris muscle partly replaced by scar tissue. Elbow and wrist-joints flexed to take tension off the nerve. The patient is recovering epicritic sensation and the function of the intrinsic muscles of the hand. The stretched motor branches to the forearm muscles have continued to functionate well. The elbow- and wrist-joints could be fully extended eight months after the operation.

way by the intermuscular septum. It is a good plan to remove that portion of the septum which is crossed by the nerve, so that the latter may not rest upon its sharp edges.

The upper-arm portion of the wound must be closed before the nerve is sutured, because, while the latter is being done, the limb must be adducted and maintained in this position during the remainder of the operation and for two or three weeks subsequent to it. It is an advantage, therefore, to use catgut in suturing this portion of the wound. The limb 


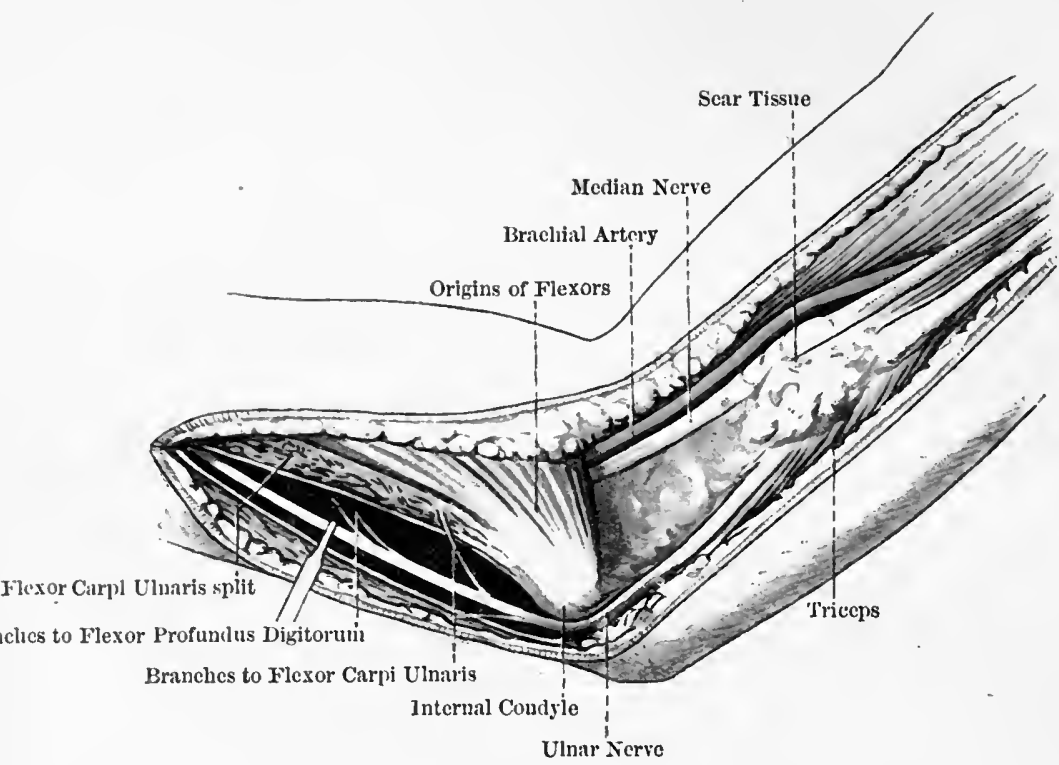

FIG. 33.-First stage of operation for a lesion of the median and ulnar nerves in the lower third of the arm. Motor branches of ulnar freed to allow of its transposition to the front of the elbow. This procedure was necessary, otherwise the elbow could not have been flexed to obtain an end-to-end suture of the median nerve.

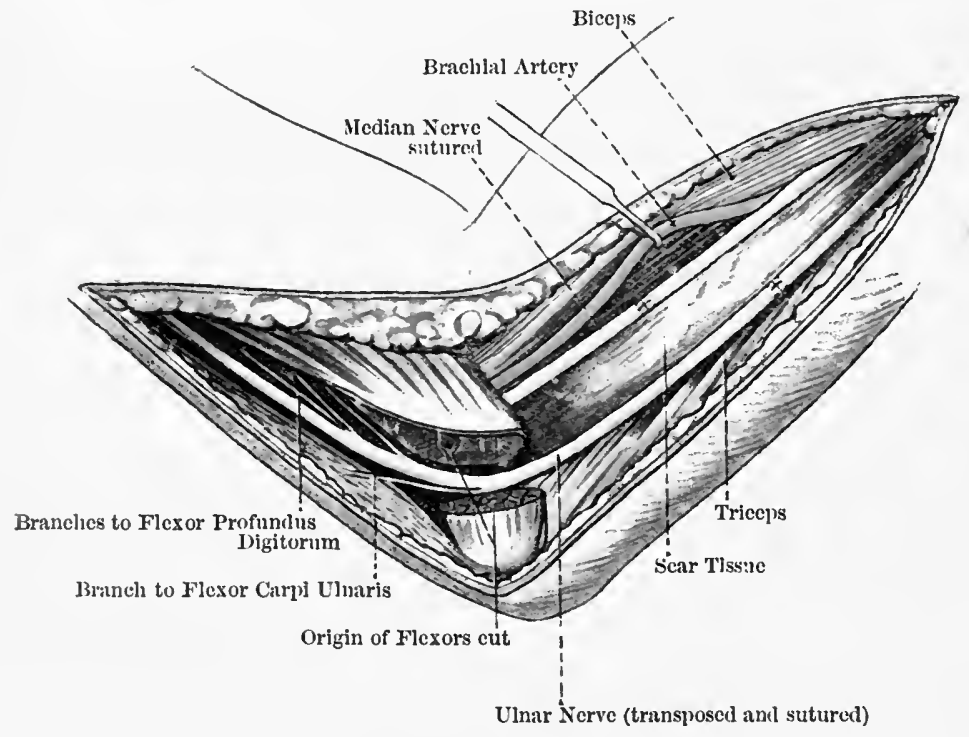

Fig. 34.-Second stage of operation in Fig. 33. The ulnar nerve has been transposed to the front of the internal condyle and both it and the median have been sutured after resection of the injured portions. Note that the motor branches of the ulnar nerve have becin made tense by the upward traction on its distal stump. Elbow flexed to relax both nerves. 
should, of course, be bandaged to the chest with the forearm fully flexed and the wrist kept in the position of ulnar flexion. The stage of rightangled abduction of the arm should not be reached until six or eight weeks from the date of operation.

When transposition is necessary for a lcsion of the ulnar nerve in the upper arm, it will be found that the gain derived by dissecting up the motor branches is much less than in a forearm lesion, as in this case the operator is not approximating the points of their origin and entry into the muscles they supply. Again, if sacrifice of the forearm branches is necessary in the case of an upper-arm lesion, it will be the distal portion of the nerve which passes through the tunnel beneath the flexor muscles; the line of suture in this case lies above the tunnel and not below it, as in a forearm lesion. (Figs. 33 and 34.)

When the line of suture happens to fall within the tunnel beneath the flexor bellies the latter must be divided in the line of the displaced nerve, which is then dropped into the trough, the muscles being then resutured over it.

In splinting the limb after the operation, adduction of the upper arm, flexion of the elbow and palmar flexion of the wrist towards the ulnar side are, when necessary, obtained respectively by means of a body bandage to fix the arm to the side, a Jones's double-gutter elbow splint for the elbow and a small single gutter splint bent to the requisite angle opposite the wrist. To maintain the physiological position of the hand and to prevent contractures, Captain Danforth, U.S. Army, has devised a small hand splint of papier mấché, which is applied to the palm and fingers and retained in position by straps and buckles; the splint is made from a plaster model of the hand held in the physiological position. Should the condition of ulnar griffe be already established or imperfectly cured, a suitable form of finger-traction splint will have to be used. (Figs. I7, p. 65 , and fig. I8, p. 66.)

\section{MEDIAN NERVE}

In Lower Two-thirds of Forearm. In dealing with the median in the lower two-thirds of the forearm, it should be remembered that immediately above the wrist the nerve is relatively superficial, whereas when traced upwards it lies deeply under the radial head of the flexor sublimis digitorum. The incision to expose the nerve should be made in the line of the flexor carpi radialis tendon. In the lower third of the forearm, the nerve is best reached by retracting the tendon outwards, but in the middle third the best access is obtained by inward retraction of the muscle; at this level, exposure is completed by dividing the radial head of the flexor sublimis digitorum near its radial attachment. In doing this the proximity of the radial vessels and nerve should be kept in mind. 
An important point to remember, too, is that the nerve clings to the deep aspect of the flexor sublimis digitorum and is liable to be retracted along with that muscle, in which case it would be sought for in vain on the flexor profundus digitorum muscle, which forms the floor of the wound. The small arteria mediana serves as an additional guide to the nerve. (Fig. 35.)

The median, when exposed by the above method, is easily traced down to the wrist. Just above the wrist it is overlapped by the flexor carpi radialis and palmaris tendons, but when these are destroyed only fascia separates it from the skin. In cases where there is much scar tissue the nerve may be drawn forwards from its bed and become closely adherent to the skin; in these circumstances the scar may be acutely painful, and it is easy to injure the nerve when making the skin incision. The palmar branch of the median pierces the deep fascia a little above the annular ligament, and it also is not infrequently involved in the scar. At the end of the operation, it is important to see that the median nerve does not lie immediately under the skin, otherwise a painful neuroma may result. This can generally be avoided by roofing over the nerve by the lower fleshy part of the sublimis, which can be stitched to the deep fascia in the neighbourhood of the tendon of the flexor carpi ulnaris.

In exceptional cases, owing to the position of the scar which has resulted from the original injury, or it may be from a previous operation, it may be necessary to expose the median in the lower half of the forearm by an incision placed nearer the medial border of the limb and then to free, retract outwards, and evert the medial border of the flexor sublimis digitorum until the nerve is reached between this muscle and the flexor profundus digitorum.

In suturing the median in the lower two-thirds of the forearm the chief relaxation is obtained by flexion of the wrist; comparatively little is obtained by flexion of the elbow, owing to the nerve being anchored by the numerous motor branches which come off in the upper third of the forearm.

The anchoring of the proximal stump of the divided median nerve by the branches which it gives off in the upper part of the forearm can, to a large extent, be overcome by dissecting them well into the muscles they supply and by freeing them up along the parent nerve to a point well above the elbow. This manœuvre is less successful in relaxing the distal portion when the lesion is in the upper arm, but even in these cases it is of some help. (Fig. 36.)

Gaps to the extent of 4 or 5 in. can be dealt with by the method described. Approximately $I_{\frac{1}{2}}$ in. are gained by free dissection, I in. by efficient freeing of the branches, and $I_{2} \frac{1}{2}$ in. by flexion of the joints. In the case of a more extensive gap, the wisest plan is to suture the untrimmed 


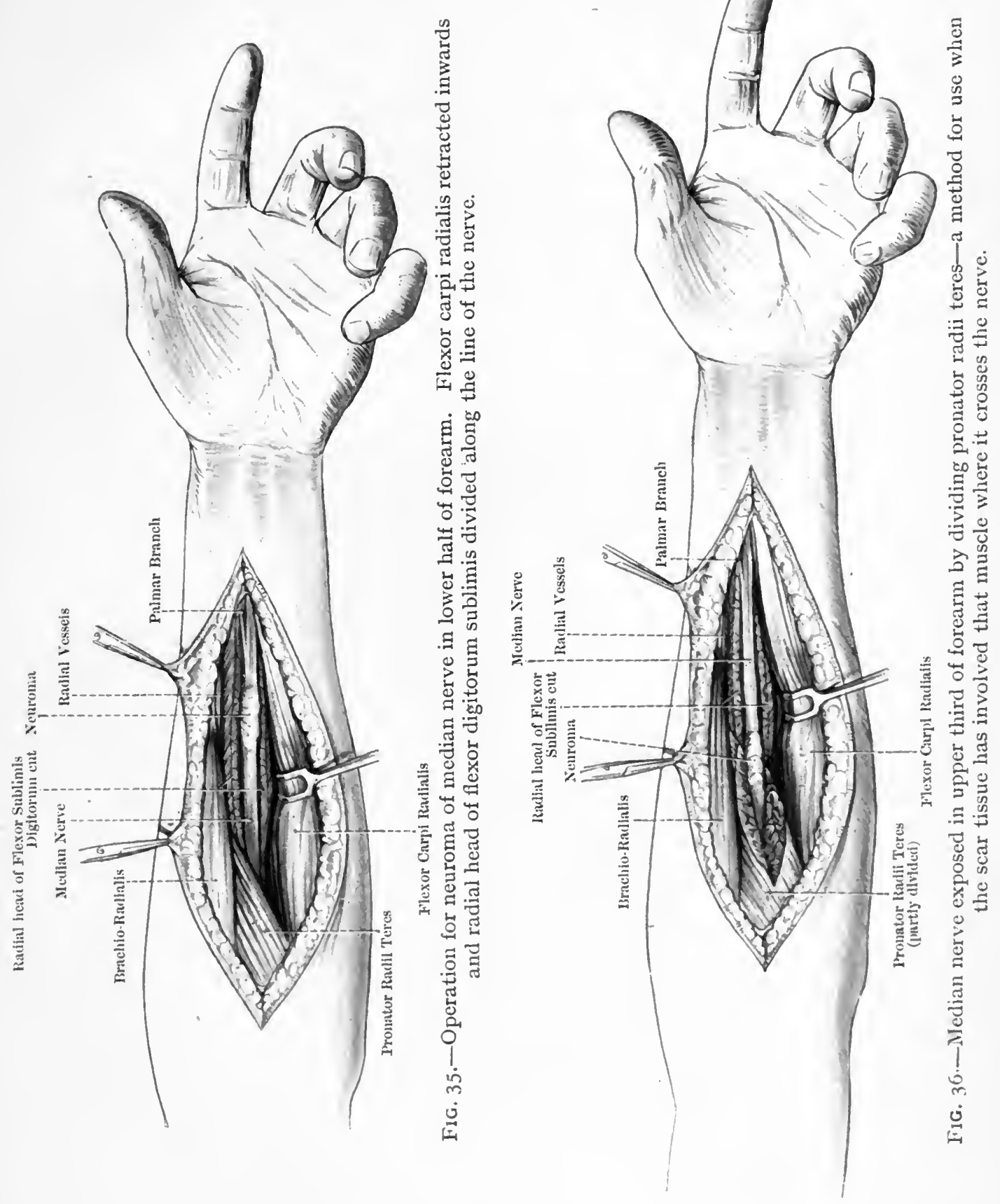


stumps of the nerve at the first operation, and after the nerve has been slowly stretched (as above detailed in chapter on General Considerations, p. 78), to re-expose the nerve at a second operation when a satisfactory end-to-end suture can generally be accomplished. This two-stage slowstretching method will in most cases obviate the necessity of resorting to nerve-grafting, \&c. The procedure is more likely to be needed when the elbow is ankylosed at an obtuse angle or even at an angle of $90^{\circ}$. The question of doing an arthroplasty of the elbow may have to be considered in such a case.

Before leaving the median nerve, reference must be made to the frequency with which it is found to be firmly adherent by dense scar tissue to the long flexor tendons, both sublimis and profundus; and not infrequently the tendons themselves are firmly adherent to one another, and one or more of them may have been completely destroyed. In these cases the freeing and suturing of the median nerve forms only a part of the operation; equally important is the freeing and repair of the tendons. All trace of scar tissue must be carefully dissected away. The tendons must be freed, and the distal stumps of those which have been completely severed must be sutured either to an adjacent intact tendon, sublimis to sublimis and profundus to profundus; or, what is sometimes preferable, the palmaris longus and flexor carpi radialis, whichever is uninjured, or both, may be severed close to the annular ligament and sutured to the distal stump, or stumps, of the divided flexor tendons. When several of the long flexor tendons have been destroyed both methods will have to be employed.

At the Elbow and Upper Third of Forearm. A single dissection will suffice to illustrate the operative technique which is called for in dealing with lesions of the median nerve in this locality. The incision is made along the inner border of the biceps in the lower third of the arm, and crosses the antecubital fossa a little internal to the tendon of the biceps, passing thereafter down the middle of the upper third or more of the forearm. It is best to find the nerve above the elbow and trace it downwards, injury to the branches being thus more easily avoided. It is usually necessary to divide and double ligature the median basilic vein just above the bend of the elbow, the internal cutaneous nerve being freed and retracted inwards. The deep fascia having been incised along the inner border of the biceps, the nerve will be found lying a little internal to the artery.

The next step is to define the outer border of the pronator radii teres, care being taken not to injure its upper branch of supply, which comes off the inner border of the median at the level of the internal condyle and runs parallel to the main trunk for a distance of about $I$ in. The belly of the pronator teres must now be powerfully retracted in a downward 
and inward direction so that the median can be followed into the upper part of the forearm. As it passes between the two heads of the pronator muscle it gives off a leash of branches, the second branch to the pronator, chiefly for the supply of its deep head, arising from the radial side of the nerve. All the other branches come off from a single bundle which can be traced up the mesial side of the nerve to about $\mathrm{I}$ in. above the bend of the elbow. From the front of this leash, branches enter the deep aspect of the flexor carpi radialis, the palmaris longus, and the flexor sublimis digitorum muscles ; from its posterior aspect passes the anterior interosseous nerve which descends behind the tendinous arch of the sublimis, to supply the radial half of the flexor profundus digitorum, the flexor pollicis longus, and pronator quadratus. It is while tracing the median beneath the superficial head of the pronator radii teres muscle that the difficulties of the operation are encountered; these difficulties are the result of the close relation of the nerve to the ulnar artery, its venæ comites, and to the profunda vein. The motor branches are best avoided by dissecting from above downwards along the outer aspect of the nerve.

To trace the nerve distal to the pronator, the incision is continued distally along the radial border of the flexor carpi radialis, which must be freed completely, care being taken not to wound the radial artery or its venæ comites where they cross the radial attachment of the pronator to disappear beneath the fleshy belly of supinator longus. The dissection is deepened by splitting the intermuscular septum between the pronator teres and flexor carpi radialis; when this has been done, the two muscles are well retracted so as to enable the operator to expose and divide the tendinous arch which connects the radial with the condylo-ulnar portion of the flexor sublimis digitorum. (Fig. 37.) With regard to the nerve supply of the flexor sublimis digitorum, its condylo-ulnar head is supplied high up by a nerve arising alone or in connexion with that to the superficial muscles. A separate branch enters the radial head, and a third branch is given off still lower for the supply of the belly to the index finger (Quain).

Owing to the depth of the median in this situation, to the number of branches given off and to its close relation to the ulnar artery, to its venæ comites and profunda vein, it is essential to obtain a good exposure of the parts. If, therefore, free retraction of the pronator radii teres fails to give sufficient exposure, the operator should not hesitate to cut the muscle across. Fortunately its main nerve of supply, which comes off from the median at the level of the internal epicondyle, enters the upper part of the muscle. This nerve should be sought for and isolated when the supero-lateral border of the muscle is being freed.

The greatest possible care should be taken to avoid unnecessary injury to the motor branches, especially those which go to supply the 
flexors of the thumb and fingers. If the lesion has already destroyed the median where these branches are given off, there should be no hesitation about continuing the dissection to any extent in both directions, provided the nerve stumps can be brought into contact without undue tension after the injured segment has been resected. The reason for doing everything possible to obtain an end-to-end suture, even although there is no hope of the patient recovering from his motor paralysis, is that there is

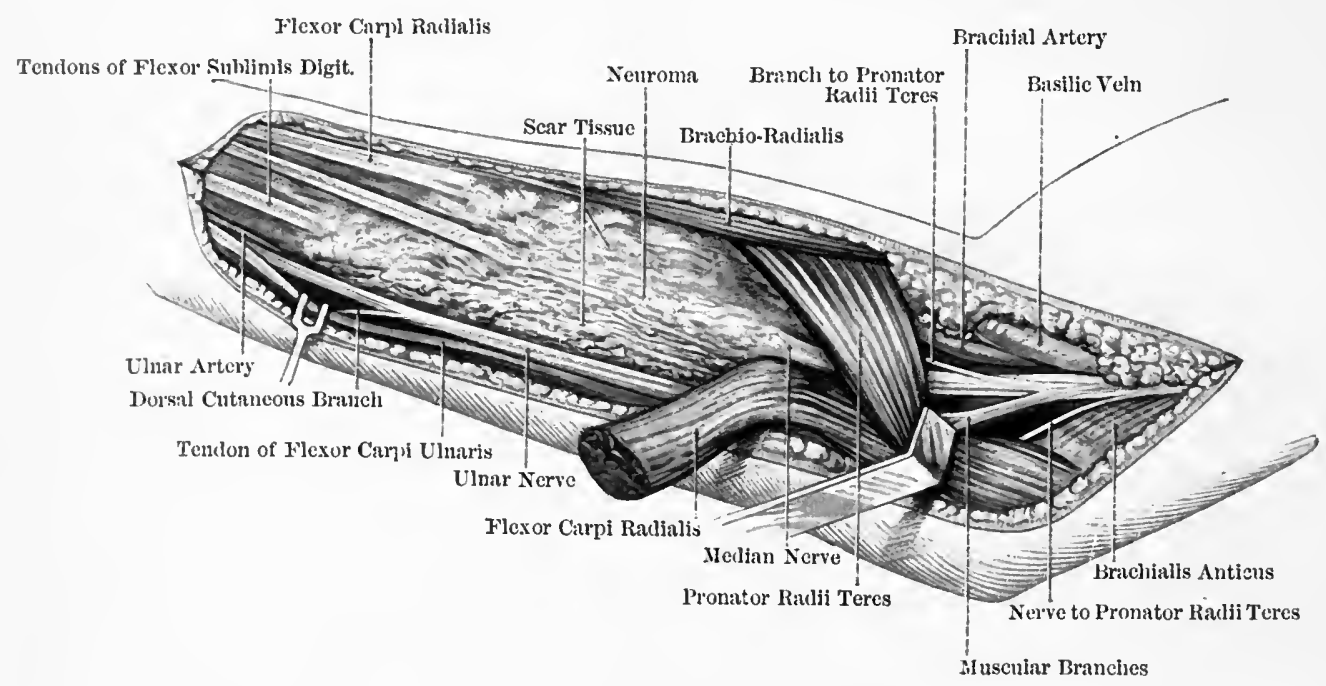

FIG. 37.-Extensive lesion of median nerve in middle of forearm. End-to-end suture was possible after freeing the branches up from the nerve and pulling its proximal end down till they became recurrent, and then flexing the wrist and elbow completely. Flexor carpi radialis sutured; tendons freed. After six months the joints extended freely, and patient was recovering power in the thenar muscles and median sensation and had the use of all the flexors.

every probability that sensation will be restored and that the trophic symptoms will disappear. Moreover, should severe neuralgia exist, this may also disappear, or at any rate be greatly relieved, by the resection. The paralysis can be subsequently dealt with by tendon transplantation.

The importance of restoring sensibility is well illustrated in injuries to the median in the lower half of the forearm. Here, although the nerve supply of the long flexors is not interfered with, the patient experiences great impairment of the motor function of the thumb, not merely as the result of the paralysis of the intrinsic muscles of the hand supplied by the median but also from the loss of sensation in the digits. If, for example, he be asked to button his coat, it will be observed that the difficulty arises from his inability to feel when he has got a proper grasp of the button. 
Hence the importance of making cvery attempt to obtain an end-to-end suture of the median nerve in spite of the fact that its motor branches may have already been destroyed. By extending the dissection from the wrist well up into the axilla the ends can be approximated after as much as 6 in. of the nerve has been destroyed.

After operations on the median nerve, the elbow and wrist are splinted in the same manner as has been described in dealing with the ulnar nerve except that there is no necessity for ulnar flexion at the wrist. With regard to the hand it is important to maintain the integrity of its transverse arches, so as to prevent stretching not only of the transverse ligaments but also of the paralysed thenar muscles. This is best done by using Danforth's splint described on p. $x \mathbf{r} 7$. If this splint is not available a leather glove may be employed; this should be provided with a strap and buckle extending transversely across the palm of the hand at the level of the middle of the first metacarpal bone, so that, by tightening the strap, the thumb is maintained in the position of opposition.

Tendon Transplantation for Median Paralysis. In cases where sensation of thumb, index, and middle fingers is preserved or curable, the lost motor function of these digits can be fairly well restored by means of tendon transplantation, \&c.

There is no muscle which can be made to act as substitute for the thenar muscles, but it has been found that even when these are completely paralysed, including the adductor pollicis, they will not be much missed if the thumb is arthrodesed at its carpo-metacarpal joint in a position of opposition and abduction, so that the angle between the first and second metacarpals is $60^{\circ}$. Radiographic examination has shown that this angle scarcely varies whether large or small objects are being grasped. It is no disadvantage if this arthrodesis results in a short fibrous, rather than a bony, ankylosis, as this allows slight flcxion at the carpo-metacarpal joint. It is a mistake to put the thumb in a position of more than moderate opposition, otherwise the hand is unable to grasp large objects. This arthrodesis opcration must precede any transplantation operation to the long flexors, otherwise the tension of the transplanted muscles will be altered by the bone operation and their function upset.

Several possible transplantations arc possible to replace the long flexors of the thumb, index, and middle fingers. The one which has been done at the Edinburgh War Hospital with success is as follows:

Into the tendon of the flexor longus pollicis at the wrist is transplanted one-half of the extensor ossis metacarpi pollicis detached as near to its insertion as possible. This ultimately gave very powerful flexion of the terminal phalanx of the thumb.

Should the middle finger, as often occurs, have its profundus supply from the ulnar nerve, the operator may confine his attention to the 
flexors of the index finger. The extensor carpi radialis longior is detached from its insertion and brought to the front of the forearm in a subcutaneous tunnel superficial to the supinator longus and then deep to the tendon of the flexor carpi radialis. It is split longitudinally, half being transplanted into the sublimis and half into the profundus tendon of the index finger, just above the anterior annular ligament. The transplantation should be done with more than semi-flexion of the interphalangeal joints, but careful after-treatment is required to prevent permanent shortening (for details v. p. I62).

\section{NERVES OF THE HAND}

In extensive lacerated wounds of the hand little or nothing can be done to repair the nerves. Operations designed for this purpose are only undertaken when the nerve lesion is strictly limited, such as may occur as the result of a clean bullet or small shrapnel wound, and especially when a foreign body is present in close contact with the nerve. The operation, which is usually done for the relief of pain and tenderness, consists generally in the removal of the missile, and if this has already been removed, it consists in the removal of the scar tissue in which the injured nerve is embedded. It is seldom possible to suture the stumps of the nerve. Captain Danforth had a case in which the deep ulnar branch of the nerve had been divided close to its origin. After removing the small area of scar tissue where the nerve had been divided, the stumps could be approximated by full flexion of the wrist.

To expose the ulnar nerve in the palm, an incision is commenced well above the wrist and carried along the flexor carpi ulnaris tendon to the radial border of the pisiform bone, and thence distally into the palm in a direction towards the interval between the ring and little fingers. The nerve is first exposed above the wrist by retracting the ulnaris tendon and in the palm by dividing the palmaris brevis muscle. The deep branch which is given off from the ulnar edge of the nerve opposite the lower border of the pisiform bone is traced to where it pierces the septum between the abductor and flexor brevis minimi digiti muscles. By splitting the septum and retracting these two muscles, the nerve may be followed and freed distally. The branches of supply to the hypothenar muscles generally spring from the deep branch of the ulnar, close to its commencement, so that if they have been injured, the chances are that the ulnar itself has also been involved. When this is the case, an attempt should be made to suture the single stursp of the ulnar to its superficial and deep divisions. In Captain Danforth's case the deep division was divided a little distal to where it gives off its branches to the hypothenar muscles. The operation was done for the purpose of restoring the function of the interossei, the two inner lumbricals and the deep thenar muscles. 
Operations on the median nerve in the palm usually form part of an operation for the freeing and repair of tendons. When the nerve alone has to be dealt with, it is invariably for the relief of painful conditions resulting from implication of its sensory branches. In the removal of scar tissue which does not involve the median or any of its branches care should be taken not to injure the digital branches to the thumb and index finger. If the operator's knowledge of anatomical detail is not equal to the occasion, he should not hesitate to refresh his memory by reference to a text-book of anatomy.

The splinting of the hand after operation has already been referred to (sce p. II7).

\section{INJURIES TO THE NERVES OF THE LOWER EXTREMITY}

\section{SCIATIC NERVE}

From the operator's point of view, lesions of the great sciatic nerve may be divided into (I) those situated in the buttock and upper part of the thigh above the point where the long head of the biceps crosses the sciatic, and (2) those situated below this level.

In the Buttock and Upper Part of the Thigh. In cases of lesions situated in the buttock and upper third of the thigh, the best access is obtained by an incision shaped like a mark of interrogation. The incision is a downward extension of the one employed by Kocher for excising the hip-joint. It extends along the outer two-thirds of a line running from the posterior superior iliac spine to the upper border of the great trochanter, thence downwards a little internal to the posterior border of the trochanter, and then downwards and inwards towards the mid line of the back of the thigh, down which it may be prolonged as far as required.

The thick subcutancous fat having been divided, the gluteus maximus is split in the direction of its fibres opposite the upper limb of the incision, whilst the lower fibres of the muscle are divided vertically a little internal to their tendinous insertion. (Fig. 38.)

The advantages of this incision are fourfold, namely (I) free exposure; (2) less interference with the nerve supply of the gluteus maximus; (3) less blecding; (4) skin wound farther away from the ano-genital region. The inner portion of the muscle is then freed from the decper structures and reflected inwards with the integuments until the nerve is reached. In doing this, care is taken not to injure the branches of the inferior gluteal nerve when dividing or applying forceps to the branches of the gluteal and sciatic vessels which enter the deep surface of the muscle. The great sciatic nerve is now exposed as it descends in the fat dorsal 
to the quadratus femoris and the upper part of the adductor magnus. Forceps are applied to any branches of the superficial divisions of the gluteal artery which have been divided. The lower border of the piriformis muscle is now defined by dividing the subgluteal fascia and separating the subjacent fatty layer with the finger or a Kocher's dissector.

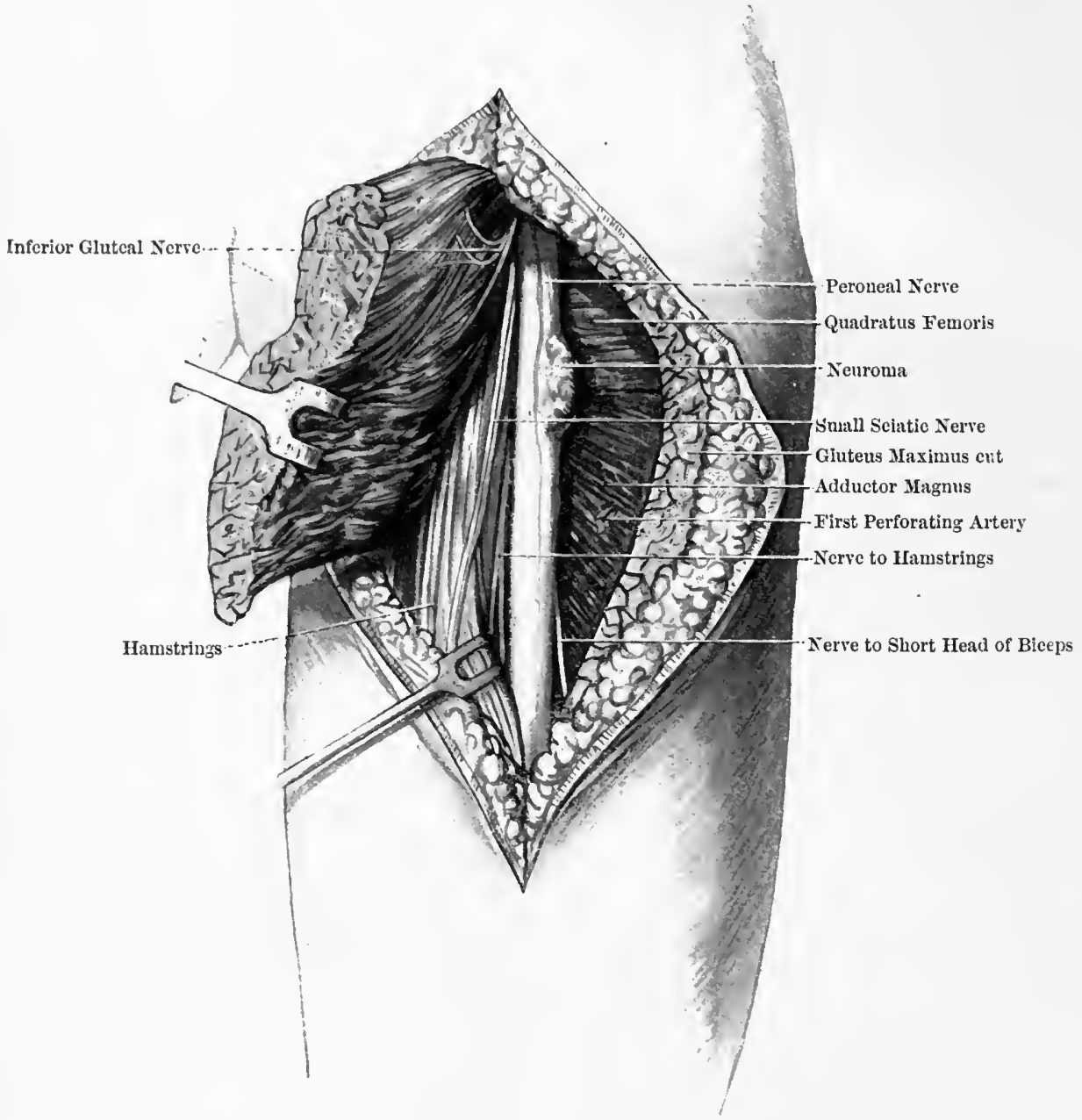

FIG. 38.-Operation for a neuroma involving the peroneal portion of the right sciatic nerve opposite the lower border of the quadratus femoris. Through the upper part of the incision the gluteus maximus has been split; below this the muscle has been divided close to its aponeurosis and reflected inwards.

In freeing and retracting upwards the piriformis muscle, the following structures will be met with immediately dorsal and superficial to the great sciatic nerve, namely the sciatic vessels and their branches, the 
inferior gluteal nerve, and the small sciatic nerve. After the vessels have been secured and the deep wound rendered as dry as possible, the above nerves are sought for and traced up to their origin from the dorsal aspect of the lowest part of the sacral plexus. If the small sciatic nerve be not involved in scar tissue, it is freed and displaced inwards. Frequently, however, it is found to be involved in scar tissue along with the great sciatic, in which case there need be no hesitation in sacrificing it, as it is a purely sensory nerve. Care should be taken, however, to preserve as far as possible the inferior gluteal nerve which often arises from the plexus in common with the small sciatic and then breaks up almost at once into a leash of branches which radiate upwards and downwards to enter the deep surface of the gluteus maximus muscle. If the trunk of this nerve is sacrificed, the whole of the gluteus maximus will be paralysed, with the result that the patient will be permanently lame, on account of his inability sufficiently to extend and abduct the thigh.

The great sciatic nerve is now thoroughly exposed and freed from the obturator internus and gemelli muscles, so that the nature and extent of the lesion may be ascertained. The involved portion of the nerve is now completely freed from adjacent scar tissue, the whole of which should be dissected away. This having been done, the nerve is dissected free as far below the lesion as may be necessary.

In order to reach the nerve above the injured segment, the piriformis muscle is thoroughly freed and retracted well upwards, and it may even be necessary to divide the muscle. The upper section may have to be made through the nerve at, or immediately above, the origins of the small sciatic and inferior gluteal nerves, that is to say, through the lowest part of the sacral plexus itself. In following the nerve upwards ventral to the piriformis muscle care should be taken to avoid injuring the pudendal nerve and the nerve to the obturator internus muscle. Sometimes, however, they are involved along with the great sciatic nerve. The nerve to the quadratus femoris muscle, which lies between the great sciatic and the ischium, is still more likely to be involved in following the nerve downward into the thigh. The upper part of the long head of the biceps is thoroughly freed and retracted well inwards. When this has been done, the great sciatic nerve will be found descending on the dorsal surface of the upper part of the adductor magnus muscle, embedded in and surrounded by adipose tissue. (Fig. 39.) Laterally the wound is limited by the deep surface of the fascia lata and the external intermuscular septum which passes from it between the biceps and the vastus externus to gain attachment to the linea aspera of the femur. In working through the fatty layer which surrounds the great sciatic nerve, several small bloodvessels will be encountered superficial to the nerve; these should be divided between forceps. They are muscular branches derived from 


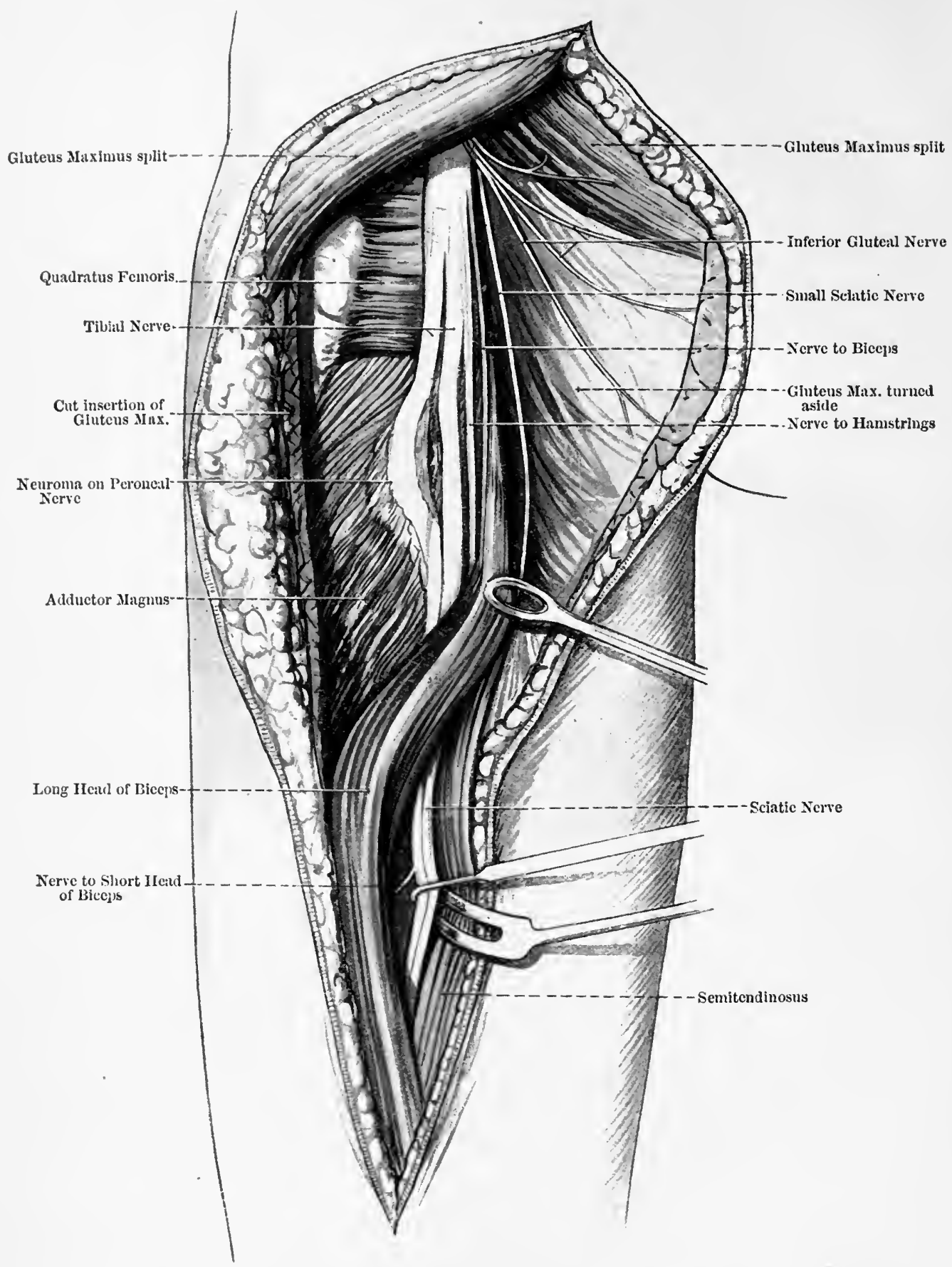

F1G. 39.-Operation for a neuroma involving the peroneal portion of the left sciatic nerve at the level of the gluteal fold. Same method of approach as shown in Fig. 38, except that the dissection has been continued distally into the thigh. The neuroma in the buttock has been exposed by retracting the biceps and semitendinosus muscles inwards; in the thigh the sciatic has been exposed between these two muscles. 
the anastomosis between the sciatic, the terminal branch of the internal circumflex, the transverse branch of the external circumflex, and the superior perforating branch of the profunda femoris. More important, however, are the branches of the great sciatic nerve which go to supply the hamstring muscles. These should be sought for and traced to their origin from the great sciatic. They all converge to join its medial border, where they often form a single trunk which can sometimes be dissected free from the injured and bulbous portion of the great sciatic as high up as the quadratus femoris muscle. By paying attention to this point, the nerves of supply to the hamstrings may be preserved instead of being sacrificed accidentally. When freeing the sciatic nerve from the adductor magnus, a sharp look-out should be kept for the branch which comes off from its ventral aspect to enter the upper part of this muscle on its dorsal aspect. Sometimes, especially when the femur has been fractured, the great sciatic is bound down to the adductor magnus by such a mass of dense scar tissue that it is impossible to preserve this branch. Fortunately, however, the adductor magnus is supplied also by the deep division of the obturator nerve.

With regard to the treatment of the operation wound the divided or split gluteus maximus is sutured with $2 \mathrm{I}$-day iodine-tannic-gut (Luken's or some other equally reliable brand), and care should be taken not to injure the muscle by tightening the sutures too vigorously. Due attention should be given to hæmostasis in order to do away as far as possible with the introduction of a drainage tube. If, as not infrequently happens, the removal of much scar tissue has been attended with venous oozing which could not be completely arrested, a tube should be introduced. In applying the dressing plenty of wool should be used so that firm pressure may be made during the application of the bandage.

In the Lower Half of the Thigh. While the great sciatic nerve usually bifurcates a little below the middle of the thigh, it may do so anywhere between the sacral plexus and the lower part of the thigh. 'Out of ${ }^{3} 8$ cases recorded by the Collective Investigation Committee the whole of the sciatic nerve emerged from the pelvis below the piriformis muscle in II 8 cases; in I7 cases the nerve left as two trunks, of which one, the peroneal, pierced the piriformis muscle; while in the remaining three the whole nerve passed through this muscle' (Quain).

An assistant should have charge of the leg, and his duty should be to see that it is held at whatever angle of flexion the surgeon finds most suitable for the particular step of the operation he happens to be concerned with. A sandbag should be placed under the lower end of the femur so as to extend the thigh a little beyond the straight. This position still further relaxes the nerve and allows also of a more thorough retraction of the hamstring muscles. The thigh should be rotated slightly inwards. 
The operation will be best described by taking as a type that which is performed for a lesion of the nerve a little above the middle of the thigh. The incision is carried along the line of the nerve from a little above the lower border of the gluteus maximus muscle to the upper angle of the popliteal space. Should the injury be at a higher or lower level

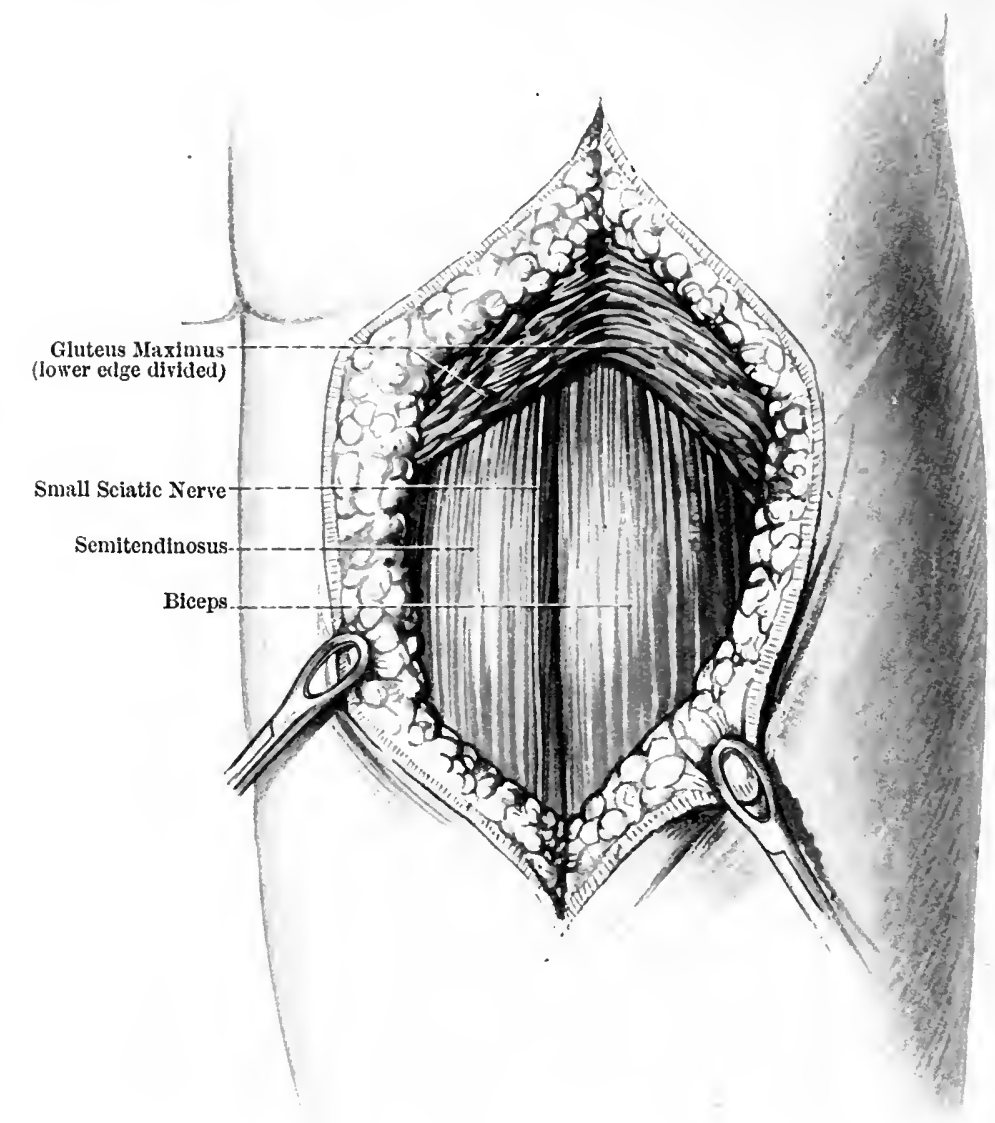

FIG. 40.-Exposure of sciatic nerve in upper third of thigh-septum found between biceps and semitendinosus.

than that selected the incision must, of course, be modified accordingly, so that the dissection may encroach either upon the buttock or upon the upper part of the popliteal space. If the incision is accurately placed, it will expose the interval between the fleshy bellies of the biceps and semitendinosus which are separated by a very delicate septum, for which the small sciatic nerve acts as a guide. When the fascia has been split, the small sciatic nerve and the terminal branch of the sciatic artery and its companion vein will be exposed. (Figs. 39 and 4r.) The septum 
between the above-named muscles is now opened up and their bellies are held well apart by suitable retractors. The great sciatic nerve lies deeply at the bottom of the wound on the dorsal surface of the adductor magnus. It is surrounded by an abundance of adipose tissue, ramifying in which are the muscular branches of the three perforating arteries of the profunda femoris and their companion veins. In applying hæmostatic forceps to

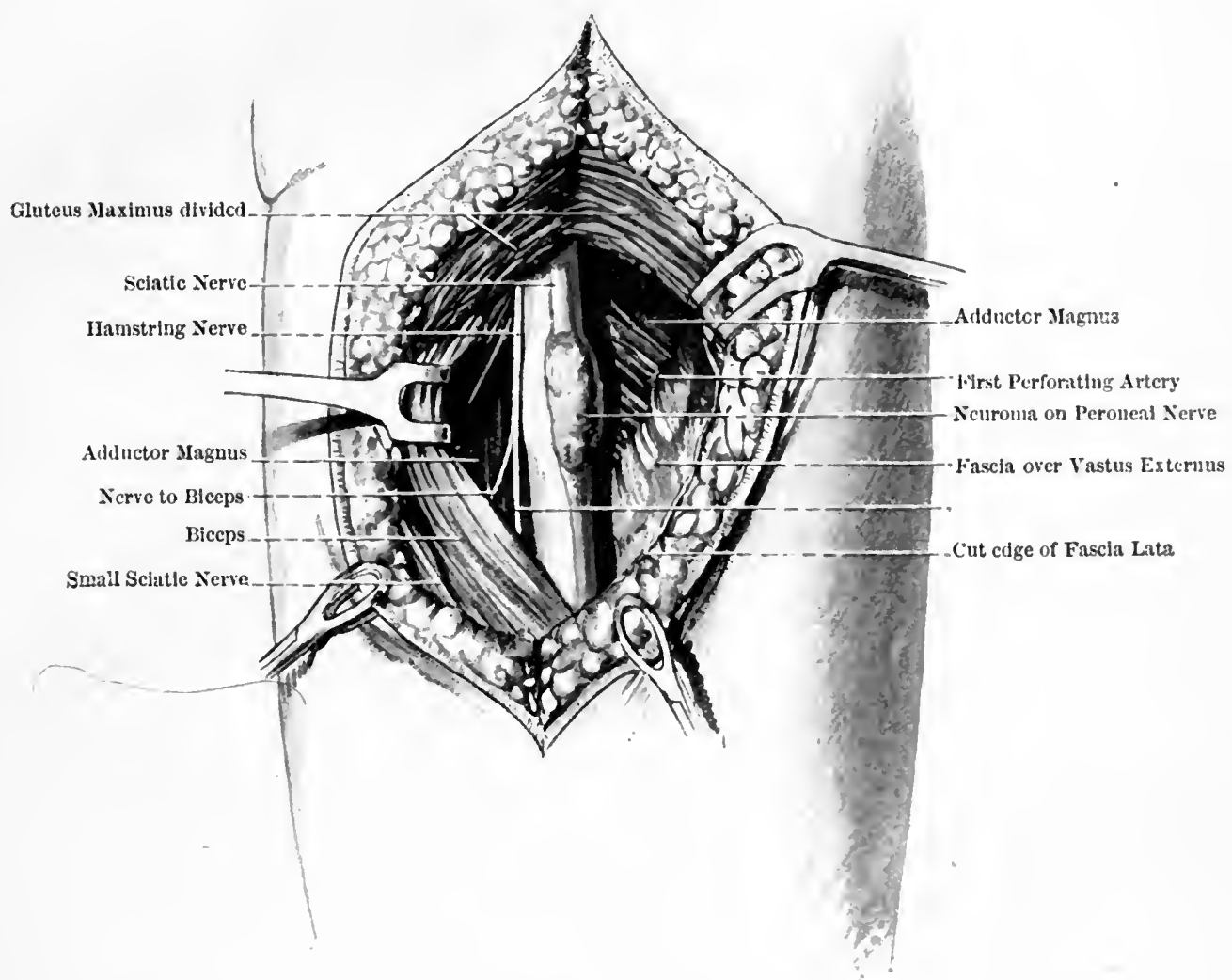

FIG. 4I.-Operation for a neuroma involving the peroneal portion of the right sciatic nerve at the upper part of the thigh. The lower border of the gluteus maximus has been divided and the biceps retracted inwards.

these vessels care must be taken not to injure the motor nerves which generally accompany the vessels and enter the muscles along with them. Again, the motor nerves are less liable to be injured if the sciatic is first exposed well above the lesion and then carcfully traced distally, a sharp look-out being kept for the branches as they arise.

The branches of the sciatic nerve to the hamstring muscles all arise 
from the medial border of the nerve (tibial portion) with the exception of the branch to the short head of the biceps, which springs from the ventral aspect of its outer border about the middle of the thigh. (Fig. 43.) The branch to the upper half of the semitendinosus and long head of the biceps comes off at the junction of the upper and middle thirds of the thigh ; the latter branch descends upon the dorsal aspect of the sciatic, which it crosses very obliquely. The branch to the lower half of the semitendinosus and to the semimembranosus comes off at a lower level.

When the lesion lies nearer the buttock, the intermuscular septum between the biceps and semitendinosus muscles is split upwards into the substance of their common origin so as to separate the tendinous portion, which belongs to the biceps, from the fleshy portion, which is associated with the semitendinosus. Before this can be done it will be necessary to repeat the lower part of the dissection just described by prolonging the incision upwards so as to expose the nerve beneath the lower part of the gluteus maximus before it descends ventral to the biceps muscle. If now the lateral border of this muscle is well freed, it can be retracted either upwards and outwards or downwards and inwards as may be necessary. When this muscle itself is badly involved in scar tissue, it may be necessary to excise a portion of it. (Fig. 40.)

Before leaving this dissection, it is advisable to warn the beginner against the error of at first mistaking the ribbon-like tendon of the semimembranosus for the great sciatic nerve. This error is quite likely to happen in exposing the nerve in the proximal half of the thigh, after splitting up the septum between the biceps and the semitendinosus muscles. When this has been done, the tendon of the semimembranosus, and not the great sciatic nerve, will be the only structure exposed at the floor of the wound. This tendon, which is about the same thickness as the nerve, lies a $\frac{1}{4}-\frac{1}{2}$ in. medial to it, both descending parallel to one another upon the dorsal surface of the adductor magnus. If the biceps be retracted well outwards, the great sciatic nerve will be exposed and the error detected. (Fig. 42.) The mistake is easily avoided if the operator will first expose the upper and outer border of the biceps and then retract it downwards and inwards; when this is done, the nerve, and not the tendon, will be the first structure exposed.

In prolonging the dissection into the upper part of the popliteal fossa, the bulky substance of the distal half of the semimembranosus is freed and retracted well inwards, while the biceps is retracted outwards. This will expose the commencement of the tibial and peroneal divisions of the nerve, one or both of which may be injured along with the lowest part of the parent trunk. The tibial division, which is considerably the larger, descends vertically dorso-lateral to the popliteal vessels in the upper part of the popliteal fossa. The peroneal division, on the other 


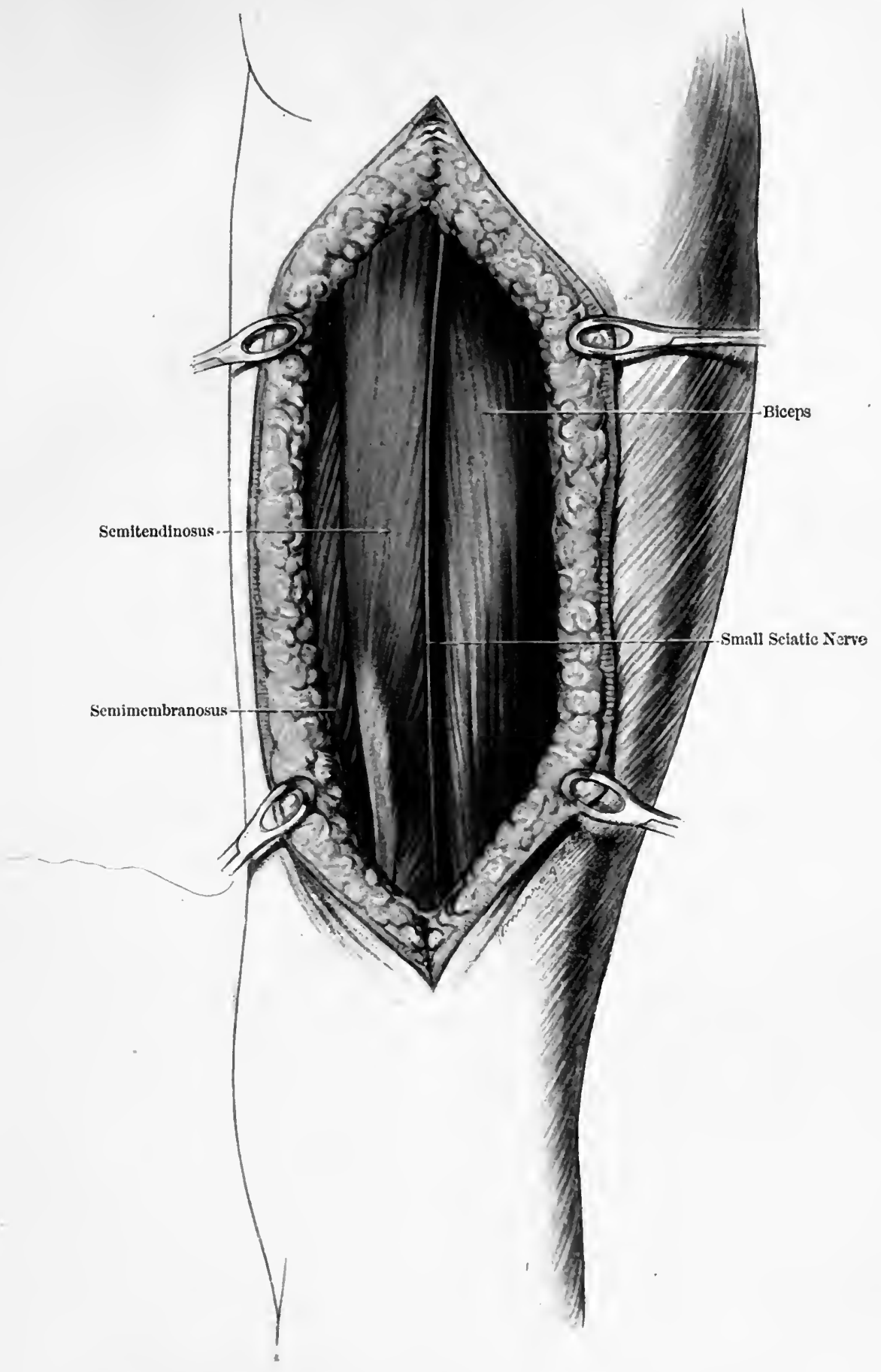

Fig. 42.-Shows first stage of dissection to expose right sciatic nerve in middle of thigh. While the N. cutaneous femoris posterior is a guide to the delicate septum between the biceps and semitendinosus muscles, the real guide to the interspace is obtained by noting the slight difference in the direction of the fibres of the two muscles. 


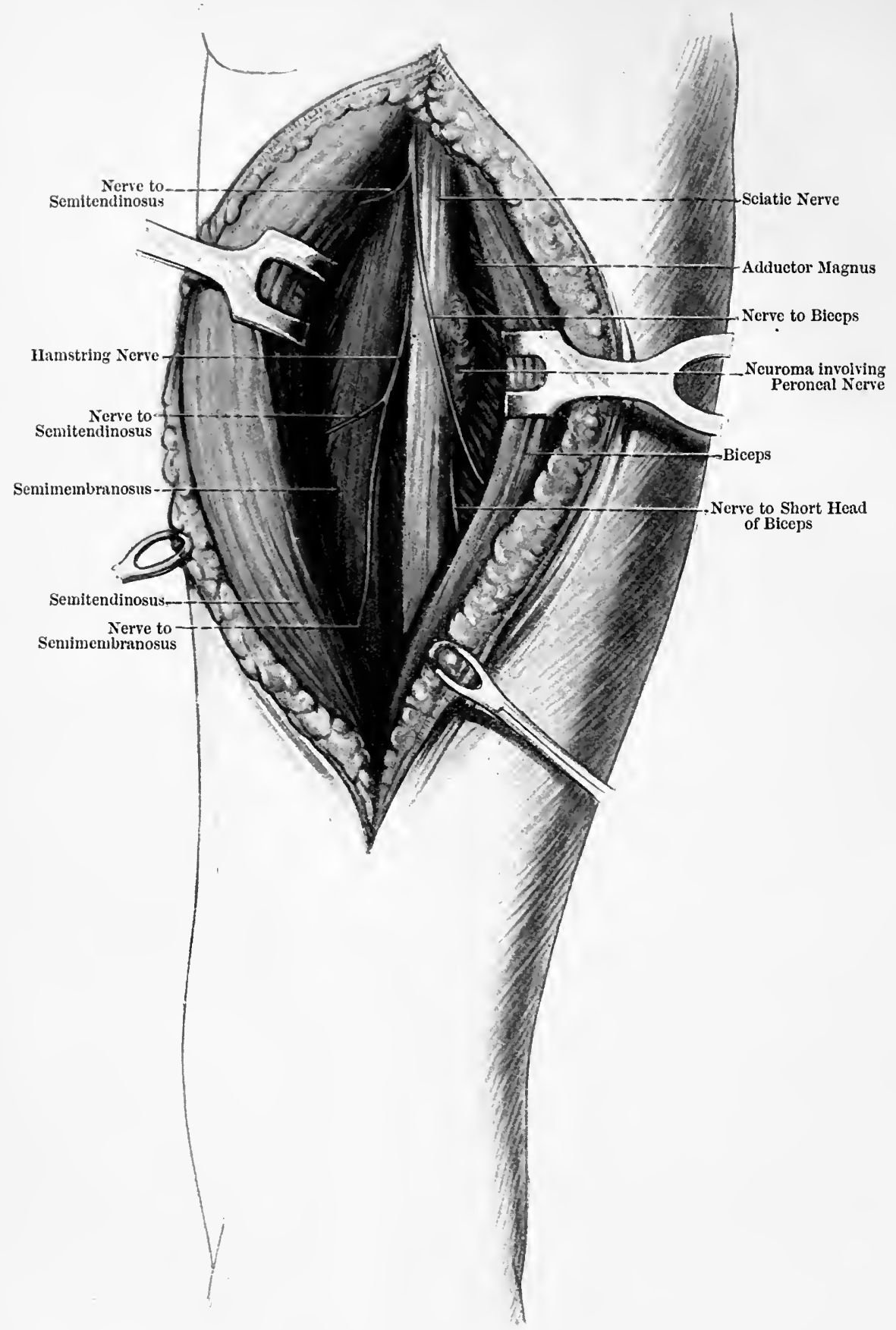

FIG. 43.-Second stage of operation shown in Fig. 4 I. Biceps retracted outwards, semitendinosus inwards. Neuroma involves peroneal portion of sciatic nerve. Nerves to hamstring muscles well seen. Note that the nerve to the long head of the biceps crosses the dorsal surface of the sciatic very obliquely, and that the nerve to the short head of the biceps arises separately from the lateral border of the peroneal portion of the sciatic. The tendon of origin of the semimembranosus muscle is seen lying medial and parallel to the sciatic nerve. 
hand, diverges towards the head of the fibula and is overlapped by the biceps tendon.

If the nerve has been sutured, the knee must be kept flexed, the degree of flexion depending on the extent of the resection. To maintain the necessary position of the joint, a Jones double-gutter knee splint is employed and a metal club-foot shoe is applied to the foot to keep it at $<90^{\circ}$.

\section{EXTERNAL POPLITEAL NERVE}

(Nervus Peroneus Communis)

In operating on the external popliteal nerve, a sandbag should be placed under the shoulder and pelvis of the same side to enable the patient to be placed in the three-quarter prone position. A third sandbag is placed under the ankle, which should be so arranged that the dorsolateral aspect of the knee is presented towards the operator. As in the case of the sciatic and internal popliteal nerves, an assistant should be detailed to maintain the leg at the necessary angle of flexion and rotation.

To lay bare the nerve, an incision is made from the upper angle of the popliteal space downwards and laterally along the medial border of the biceps to end a little below the neck of the fibula. When the popliteal fascia has been split, the proximal end of the nerve is exposed by freeing and retracting the lower part of the fleshy belly of the biceps, which overlies the nerve. The nerve is then exposed as it crosses the plantaris muscle. Lower down it crosses the outer head of the gastrocnemius, and in this situation it is very closely applied to the inner border of the biceps tendon. Both structures lie immediately beneath the popliteal fascia, which is here so thick and dense as to be almost aponeurotic in character. In many cases the injury has caused the nerve to be so firmly adherent to these structures by dense scar tissue that some difficulty may be experienced in isolating it, and not infrequently, the nerve is found to be completely destroyed for 2 or 3 in. When this extensive destruction extends so low down as to involve the commencement of its three divisions (recurrent tibial, musculo-cutaneous, and anterior tibial nerves) in the substance of the peroneus longus muscle, all idea of an end-to-end suture must be abandoned. Fortunately, the drop-foot which results from the paralysis of the extensor and peroneal muscles can be very satisfactorily dealt with by the operation of tendon suspension of the foot, a description of which will be found on p. 175 . The communicans fibularis nerve, which descends vertically between the popliteal fascia and the outer head of the gastrocnemius, will frequently have to be sacrificed. This is a matter of no importance as it is a purely sensory nerve. 
Provided a good distal stump of the external popliteal can be obtained, suture can be done in cases where there is a considerable gap between the rerve ends, because good relaxation can be obtained by flexing the knee and tracing the nerve up the thigh, if necessary as high as the buttock. In doing this it can be separated from the internal popliteal portion of the sciatic for the whole distance by splitting their common sheath. The only branch given off in the thigh from the external popliteal portion is one to the short head of the biceps, so that there is no contra-indication to this extensive dissection.

Fixation of the limb after operation is obtained by the methods employed for lesions of the sciatic nerve. When the knee has been extended and the patient begins to walk, it is necessary to fit the boot with an outside iron and inside T-strap, a stop-joint preventing plantar flexion of the foot.

\section{INTERNAL POPLITEAL NERVE}

In spite of the close relation of the internal popliteal nerve to the popliteal vessels, it is not infrequently injured without the vessels being involved. The injury may be due to a shrapnel wound which has only penetrated as far as the nerve or to a missile passing transversely through the popliteal fossa close to its roof. In other cases the nerve is compressed and stretched over the wall of a popliteal aneurism.

Exposure of the nerve in the upper part of the space has been dealt with in the description of operations on the sciatic in the lower third of the thigh. To expose the nerve in the middle and lower divisions of the fossa, an incision is commenced over the centre of the posterior aspect of the thigh at the junction of its lowest and middle thirds, and continues vertically downwards to the junction of the upper and middle thirds of the leg. The popliteal fascia is now split along the whole length of the fossa, and any portion of it which has become adherent to the subjacent structures should be dissected away. The external saphenous vein, which ascends in the superficial fascia covering the lower half of the space, should be freed and divided between forceps. If it is involved in scar tissue, care should be taken not to wound it just where it joins the popliteal vein. No attention need be paid to the terminal branch of the small sciatic nerve or to the communicans tibialis and communicans fibularis nerves as they are purely sensory, and if they are embedded in scar tissue it is better that the involved portions should be excised. The communicans tibialis nerve descends in the furrow between the two heads of the gastrocnemius, while the communicans fibularis descends on the dorsal surface of the outer head of that muscle. If these nerves are not involved in scar they are easily freed and drawn aside. The nerves to the gastrocnemius, one of which enters the dorsal surface of 
each head of the muscle about 2 in. below its origin, along with its companion muscular artery, should, on the other hand, be preserved if possible. Traced upwards, these branches are found to be given off from the internal popliteal at the level of the upper border of the femoral condyles. It follows, therefore, that, when the lesion of the main nerve occupies the lower part of the fossa, these motor branches are not necessarily involved, and that by careful dissection they may often be preserved intact. (Fig. 44.)

The next step in the operation is the exposure and frecing of the internal popliteal nerve below the lesion. To do this, the two heads of the gastrocnemius muscle must be separated and well retracted, the plantaris muscle being freed and displaced along with the outer head. The nerve to the soleus is now seen descending immediately upon and dorsal to the internal popliteal trunk close to the medial border of the plantaris. At a little lower level, the muscle passes downwards and inwards between the two nerves. The branch to the soleus, however, is so closely related to the parent trunk that it is almost certain to be involved along with it. The plantaris muscle itself may be so implicated that a part of it may have to be resected.

The lowest part of the dissection involves the cutting across of the tendinous arch of the soleus as it spans the poplitcal vessels and internal popliteal nerve, where they become continuous with the posterior tibial vessels and nerve. It is just at this level that the posterior tibial nerve gives off a muscular trunk which, after passing beneath the arch, immediately breaks up into a leash of branches for the supply of the three deep muscles of the calf. The highest branch supplies the tibialis posticus, which explains the escape of this muscle in some cases of complete division of the posterior tibial nerve high in the calf. If the lesion is at the lowest part of the popliteal space, the soleus must be split in a downward direction as far as is necessary (v. Posterior Tibial Nerve).

The most troublesome part of the dissection is generally the separation of the injured portion of the ncrve from the subjacent popliteal vessels, more especially from the vein. In the middle of the space the nerve lies superficial (dorsal) to the vein; while at the lowest part of the space it lies dorso-medial to the popliteal vessels. These vessels, even in the normal condition, are closely bound together in a common sheath. Fortunately the vein is almost as thick-walled as the artery. Troublesome bleeding is liable to occur when the vessels are implicated in the scar tissue. The vein may have to be ligatured, but great care should be taken to avoid wounding the artery.

A drain may have to be introduced down to the floor of the space, but it should be omitted if possible. If the popliteal fascia is intact it may be brought together by a few interrupted catgut sutures; frequently, 


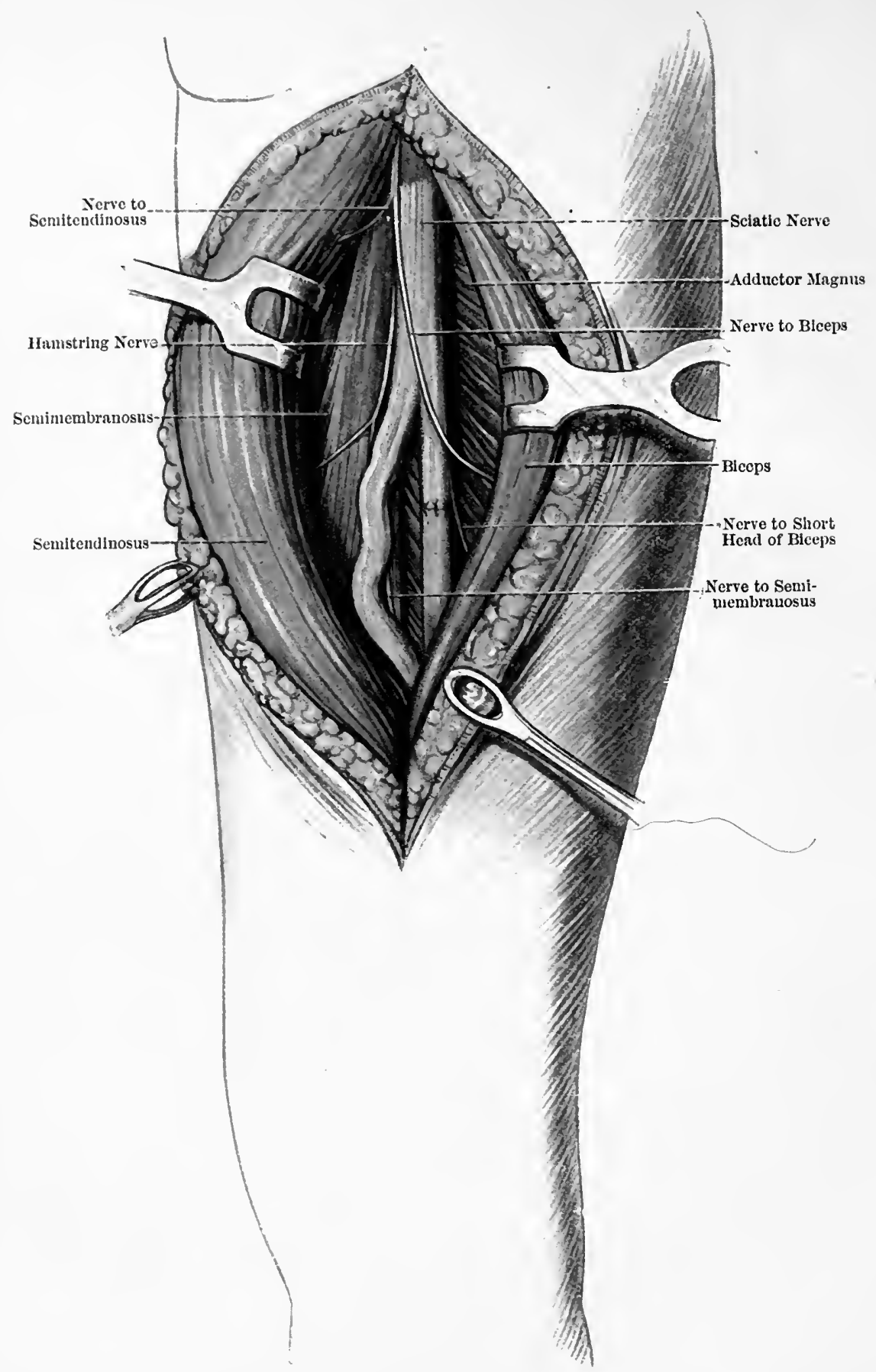

FIG. 44.-Third stage of operation shown in Figs. $4 \mathrm{I}$ and 42 . The sciatic nerve has been split longitudinally, the neuroma which involved its peroneal portion has been resected and the stumps sutured with fine linen thread. Note the apparent lengthening of the tibial portion of the nerve. 
however, enough of the fascia has to be removed to prevent its edge from being approximated.

Fixation of the limb after operation is obtained by the methods employed for sciatic nerve lesions. When the knee has been extended and the patient begins to walk, his boot should be fitted with an inside iron and an outside T-strap ; the iron should have a stop-joint to prevent dorsiflexion of the foot.

\section{POSTERIOR TIBIAL NERVE}

In the writer's experience the posterior tibial nerve has furnished only 6 per cent. of the operations on the nerve trunks of the lower extremity. While this is the case, it should be remembered that patients themselves often do not notice partial anæsthesia of the foot and weakness of its intrinsic muscles, as they would do at once in a corresponding lesion of the hand. The objective signs, such as clawing, trophic ulcers and atrophy of the sole muscle, are late in developing and are very variable in their degree of severity, so that in some cases of complete division of the nerve, they will only be noted after careful examination. If all cases of wounds of the leg are examined for symptoms of nerve injury within the first two weeks, some involvement of the posterior tibial will be found in a large proportion of cases. In many, of course, spontaneous recovery occurs.

In the upper two-thirds of the leg, the posterior tibial nerve is deeply placed beneath the gastrocnemius and soleus muscles, where it occupies, along with the posterior tibial vessels, a special compartment of the fascial septum which intervenes between the superficial and deep muscles of the calf of the leg. While the nerve, like the artery, may be approached either through a mid-dorsal or an internal incision, there can be no question that in the case of the nerve, the former is much the better route as it allows the nerve to be more directly and more freely exposed and to be more easily drawn up into the wound during the suturing. Much of the difficulty will be overcome if the operator does not hesitate to make the wound of sufficient length to allow of adequate retraction of the superficial muscles.

The incision should extend from a little below the middle of the popliteal space down the mid-dorsal line of the limb with a slight deviation inwards as far as the junction of its middle and lowest thirds. The terminal branch of the small sciatic nerve may be ignored, but the other structures which occupy the line of the incision, namely the external saphenous vein, the communicans tibialis, and communicans fibularis nerves, which, at the lower part of the wound, have joined to form the external saphenous nerve, are freed and drawn outwards or, if involved in scar tissue, are 
resected. The incision is deepened in the upper part of the wound in the interval between the two heads of the gastrocnemius, and below this level the muscle is split down the middle and the cut is prolonged still farther downwards, so as to bisect the tendinous portion to within a few inches of the heel. As these cases are not infrequently complicated by equinus, this procedure allows the surgeon to complete the operation by a Z-shaped lengthening of the tendo Achillis. The two halves of the muscle having been retracted, the wound is deepened so as to split also the subjacent soleus muscle, care being taken to preserve the motor nerve which enters its dorsal aspect close to the upper border of its outer head. In splitting the muscle, the operator should bear in mind its great thickness, and when the knife has penetrated half its depth, he should be prepared to see the flattened tendinous intersection which occupies the whole width of the muscle. The fibres which spring from the dorsal aspect of the intersection pass downwards and backwards, while those which spring from its ventral aspect proceed downwards and forwards, the result being that along the middle of the cut surface of the muscle there is exposed the cut edge of the tendinous insertion with fibres radiating from it in a bipenniform manner. It is necessary to call special attention to this tendinous sheet in the substance of the muscle, otherwise the operator is liable to mistake it for the fascial layer which lies beneath the muscle and covers the deep flexors. When the whole thickness of the soleus and the upper part of the tendo Achillis has been split, the two halves are firmly retracted so as to expose the above-mentioned fascia. (Fig. 46.) By splitting this fascia a little internal to the medial border of the flexor longus hallucis muscle, opposite the middle of the calf, the posterior tibial nerve will be at once exposed with the posterior tibial artery and its venæ comites lying in close relation to its medial edge. The nerve is now freed in both directions. In doing this, some trouble may arise from the muscular branches of the posterior tibial vessels, and especially from the veins where they join the venæ comites. When the upper part of the nerve is reached, it will be found to lie in the interval between the posterior tibial artery and its large peroneal branch, while higher up still it lies directly upon, that is to say, dorsal to, the posterior tibial artery just where the peroneal artery is given off. It is here especially that the field of operation may be obscured by bleeding from the venæ comites. The peroneal vessels are so closely applied to the bone that if they are injured they are difficult to secure except by a stitch. To follow the nerve upwards into the popliteal space, the tendinous arch at the upper border of the soleus must be divided. The motor nerves to the deep muscles of the calf frequently spring from a common trunk which arises a little above this arch. The main branch to the flexor longus hallucis accompanies the peroneal artery ; a small branch comes off at a lower level for the supply 
of the distal half of the muscle. The tibialis posticus also receives a second branch in the lower half of the leg. If the nerve is firmly adherent in scar tissue, either the posterior tibial or the peroneal artery may have to be ligatured. As the wound is a very deep one, all bleeding should be carefully arrested before the nerve is sutured. It may be necessary to

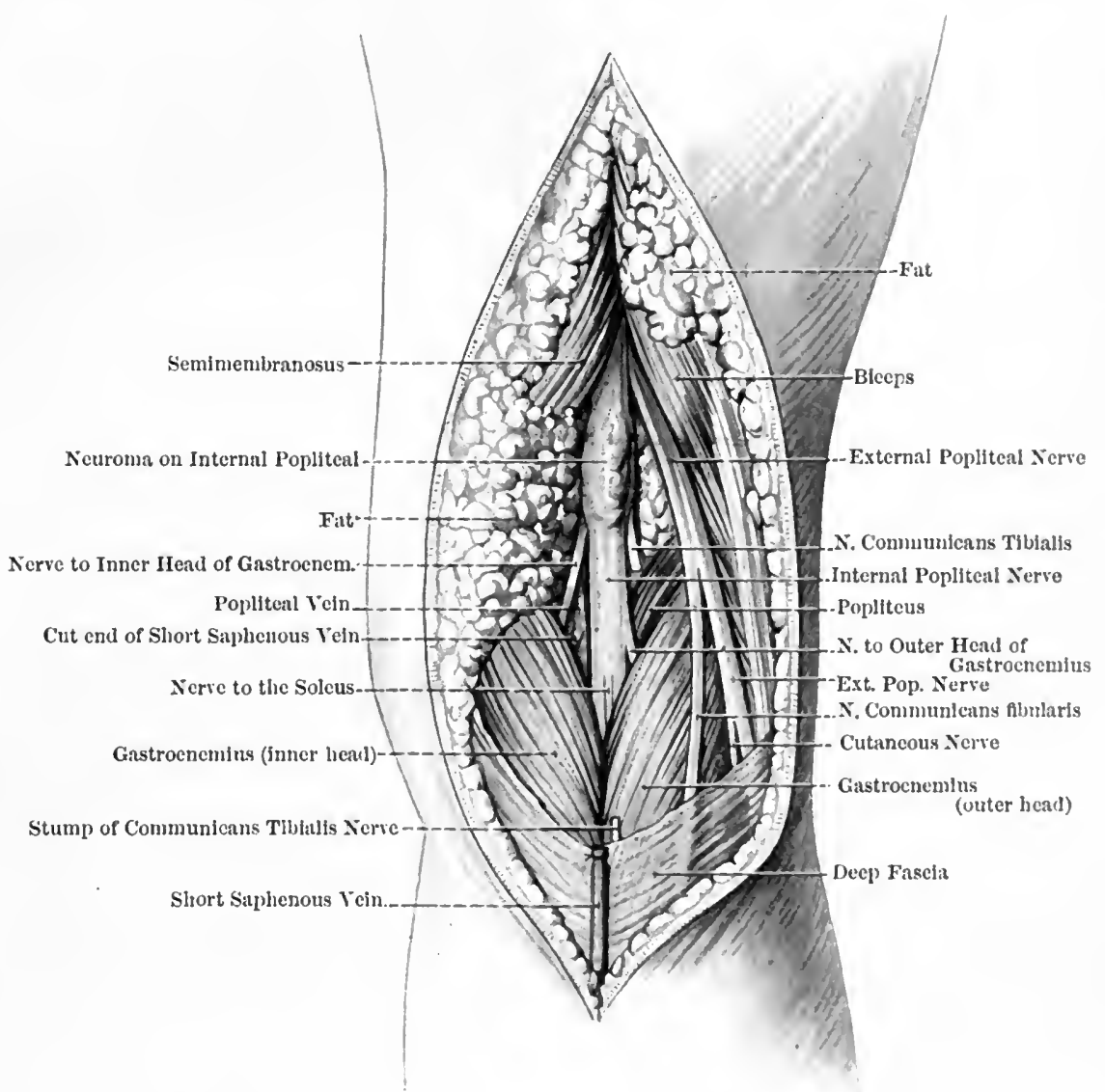

FIG. 45.-Operation for neuroma of internal popliteal (tibial) nerve.

introduce a drain. In the upper part of the wound, the split soleus and gastrocnemius muscles are sutured independently; while in the lower half, where they join to form the tendo Achillis, only one row of sutures is employed.

If much of the nerve has been resected it will be necessary to plantar flex the foot as well as flex the knee. If the foot is brought gradually back to a right angle before commencing to straighten the knee, there will be no necessity subsequently to lengthen the tendo Achillis to allow 


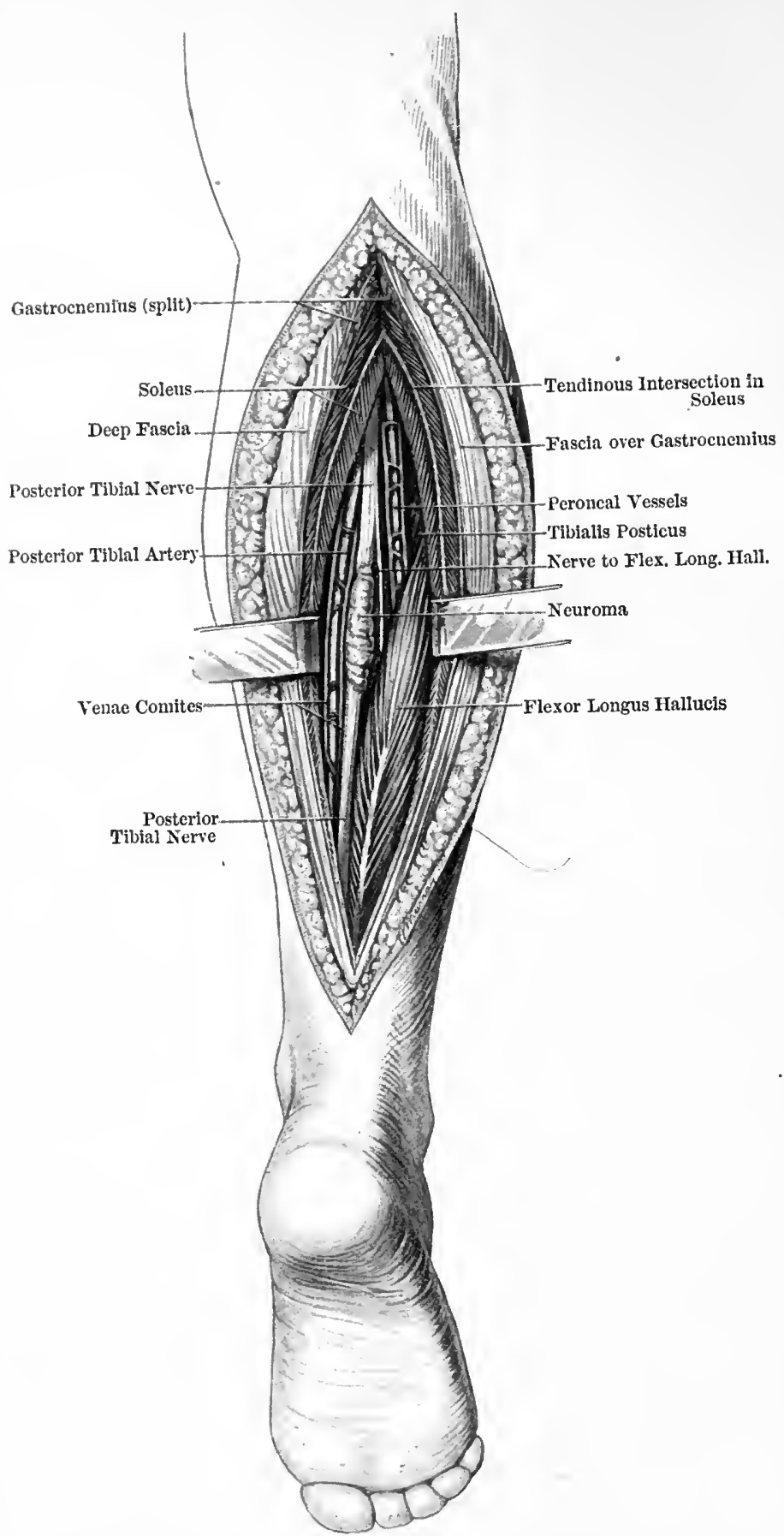

FIG. 46.- Operation for the exposure of a lesion of the posterior tibial nerve in the middle third of the leg. Gastrocnemius and soleus muscles split through a long median dorsal incision; tendinous intersection of soleus well seen. Note the relation of the posterior tibial nerve to the main vesscls and to the flexor hallucis longus muscle. 
the foot to be dorsiflexed after the nerve is united. A 3-in. gap can be overcome by flexion of the knee alone if the internal popliteal nerve is freed up into the lower third of the thigh, care being taken to dissect up the branches to the superficial muscles of the calf.

In exposing the posterior tibial nerve in the lowest third of the leg the incision should run parallel to the medial border of the tibia midway between it and the tendo Achillis. The internal saphenous vein and nerve lie in the subcutaneous fat at the medial edge of the wound. After the strong deep fascia has been divided, a still deeper layer is exposed; it extends laterally between the tendo Achillis and the deep muscles. To lay bare the posterior tibial nerve, the operator should keep the edge of the knife directed towards the latter muscles so as to divide this deeper layer of fascia which forms their immediate covering. The incision should be made behind and lateral to the neuro-muscular bundle so as to avoid injury to the venæ comites which lie internal to the nerve. In the upper part of the wound; the fleshy medial border of the soleus must be retracted well outwards, when the nerve will be found in the interval between the adjacent borders of the flexor longus digitorum and the flexor longus hallucis muscles, both of which are here still fleshy. The tibialis posticus muscle, being overlapped by the above muscles, does not come directly into view. Near the ankle the flexor longus hallucis is still fleshy, while the tibialis posticus and flexor longus digitorum muscles are represented by their tendons, both of which lie antero-internal to the nerve in close relation to the medial border of the tibia. The posterior tibial vessels lie between the two tendons and the nerve, but closer to the latter. The calcanean branch, which supplies the heel and adjacent part of the sole of the foot, should be spared if possible; it arises from the posterior tibial a little above the upper border of the internal annular ligament.

\section{NERVE INJURIES IN THE FOOT}

Operations for nerve injuries in the foot are less frequently necessary than in the case of the hand. If the original injury is extensive, operative treatment of the nerve lesion per se is not practicable. In localized lesions, operation may be called for to relieve pain or tenderness due to the implication of the nerve in scar tissue or for the removal of a foreign body-a piece of khaki cloth in one case-which has implicated the nerve.

In other cases, exploration is indicated for the cure of a trophic ulcer. The plantar nerves may be implicated at or near their origin, and in this situation a successful resection may sometimes be carried out. The branches to the toes seldom call for operative measures except when they are involved in a painful scar.

To expose the terminal portion of the posterior tibial nerve and the commencement of the plantar nerves, an incision is begun well above 
the ankle-joint and carried down nearly one finger's-breadth behind the posterior border of the internal malleolus and thence across the abductor hallucis muscle to the internal intermuscular septum. The internal annular ligament and deep fascia having been divided in the line of the incision, the posterior tibial nerve is found lying between the posterior tibial vessels and the tendon of the flexor longus hallucis. To follow the plantar nerves into the sole, the upper and lower borders of the abductor hallucis muscle are freed and the muscle is either retracted downwards or completely divided, according to the position and extent of the lesion. To expose the internal plantar nerve farther forwards the incision is made along the line of the internal intermuscular septum.

\section{ANTERIOR CRURAL NERVE}

It may be stated at once that complete paralysis of the anterior crural nerve is rarely met with, its short course in the thigh as an individual trunk accounting for this. Again, a wound of any severity in this locality would be very apt to cause damage to the femoral vessels, amputation or death from hæmorrhage resulting. Incomplete paralysis from a grazing type of wound is the more usual occurrence from injury to one or more of the individual muscular branches; again, in certain cases of extensive laceration of muscles with subsequent scar formation on a large scale, the direct muscle involvement may be as much responsible for the clinical findings as the injury to the nerve itself.

While exploration of the nerve is indicated for a partial lesion which is not recovering or for the relief of pain, actual resection is rarely possible on account of the leash of branches into which the nerve breaks up. Transplantation of the biceps cruris and gracilis or semitendinosus, preferably the former, into the periosteum of the patella is the best procedure in such cases.

\section{PROCEDURES WHEN END-TO-END UNION CANNOT BE EFFECTED}

\section{NERVE GRAFTING}

When all efforts to obtain end-to-end suture have failed, the gap may be bridged by means of a portion of a sensory nerve, the largest available being used, either as a single or a multiple cabie. The internal cutaneous of the arm may be sacrificed and in some cases its calibre is almost equal to that of the ulnar. Unfortunately, it is usually injured by the same lesion which destroyed the median or ulnar; it may also bifurcate at a higher level than usual, in which case the trunk may be too short. The 
dissection for the exposure of the internal cutaneous nerve is described along with operations upon the median and ulnar nerves in the upper arm.

A nerve which is very suitable for grafting purposes is the cutaneous portion of the musculo-cutaneous nerve in the leg. It is exposed by an incision along the middle third of a line extending from the anterior border of the head of the fibula to a point one finger's-breadth in front of the external malleolus. This incision exposes the septum between the peronei and extensors; the nerve, after piercing the septum very obliquely, becomes subcutaneous at the junction of the middle and lower third of the leg. The nerve measures about 6 in. from the point where it leaves the motor branches to the point where it bifurcates. Quite exceptionally it may be convenient for grafting purposes to utilize either the external cutaneous nerve of the thigh or a portion of the long saphenous nerve below the knee.

The graft is fixed in position by means of one or two retaining sutures of No. I6o linen thread which pass through the sheaths of the graft and prepared stumps. Care is taken that the graft is not subjected to undue strain.

Only six nerve-grafting and two flap operations have been done at the Edinburgh War Hospital and particulars of these are given in the table on p. 146 .

In one case, where there was only the scantiest evidence of nerve regeneration, the nerve was explored again and the graft was found to be shrunken, and flattened and fibrous-looking. The whole graft and its junctions with the ulnar were removed and prepared for microscopic examination. A fresh graft from the musculo-cutaneous of the leg was put into the ulnar.

In one of the two cases in which satisfactory return of function has occurred after nerve grafting (i.e. into the median), the question of an abnormal ulnar distribution cannot be wholly excluded. The clinical evidence in all the other cases points to an arrest of the advance of the growing axones at the distal junction of the graft with the main nerve which could easily be explained by a development of scar tissue at the distal suture line before the axones reached it. If this be the case, it would be a logical procedure to explore the distal junction of all grafts when the distal tingling has ceased to extend beyond it. The further progress towards recovery is no doubt due to the fact that the growing axones have been unable to penetrate the sclerosed barrier which has developed at the distal junction.

The partial but very incomplete recoveries indicated in the table are accounted for by the successful penctration of a few nerve fibres through the sclerosed distal junction. 

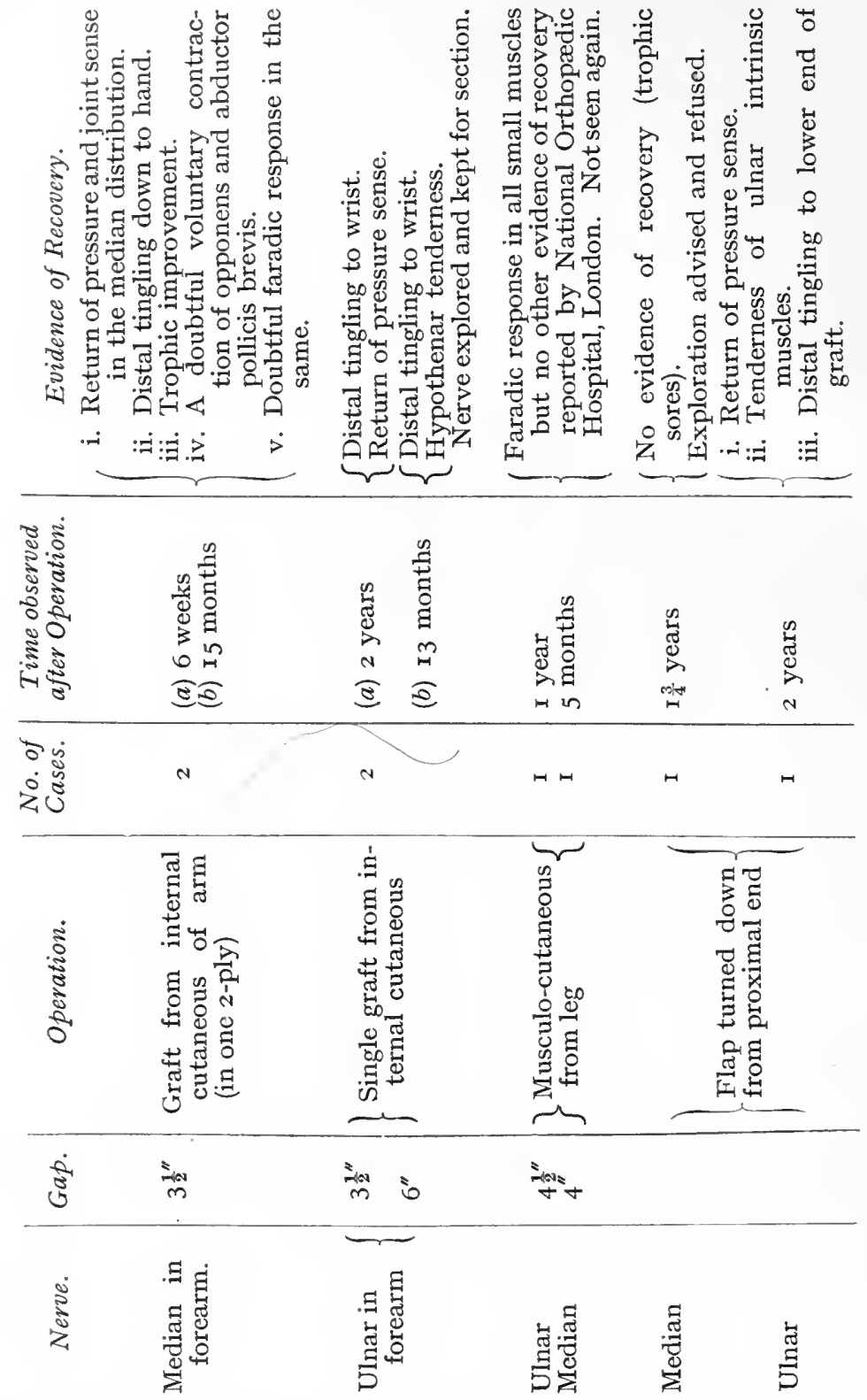

The alternative procedure, of turning down a flap from the proximal end of the injured nerve, is not to be recommended because it increases the distance which one-half of the growing axones have to traverse and it fails to provide a bridge equal in calibre to that of the injured nerve. To turn up a flap from the distal segment is not advisable if it entails, as it frequently would, the sacrifice of important branches. 
In cases of very extensive lesions of the sciatic, especially where stiffness of the knee-joint prevents relaxation of the nerve by flexion of that joint, it may be advisable to bridge the more important tibial portion of the nerve by sacrificing a corresponding length of the peroneal portion for use as a graft ; in some cases a double-ply graft can be taken from the peroneal portion, half being obtained above, and half below the lesion. This is desirable as the tibial portion is usually twice the diameter of the peroneal nerve. The permanent foot-drop resulting from sacrifice of the peroneal portion of the nerve can be treated by a foot-slinging operation, done later. The sensory loss from destruction of the peroneal nerve is

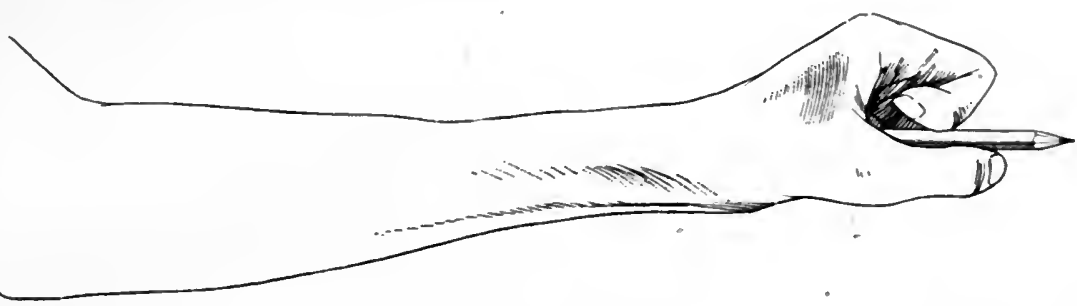

FIG. 47.-To illustrate functional disability in combined paralysis of lumbricæ and interosseous inuscles in cases where median and ulnar nerves are both involved.

of little importance. Everything should be done to secure recovery of the tibial portion of the nerve, as its paralysis is serious, from its tendency to be complicated by disabling trophic ulcers on the sole of the foot.

\section{BONE SHORTENING}

Shortening of the femur has been done as an adjuvant to approximation, where stiffness of the joints in extension prevents immediate relaxation or slow stretching of the nerve. Stiffness of neighbouring joints is a serious complication of extensive nerve injuries. When the elbow is the joint involved, a preliminary arthroplasty is sometimes advisable. This is not to be considered in the case of the knee, but, as an alternative, shortening of the femur is justifiable in certain cases.

Sir Robert Jones recommends a transverse osteotomy followed by lateral displacement of the fragments. Later on, when recovery is advancing, the bone is gradually lengthened again.

In most cases, shortening of the humerus is to be preferred to an arthoplasty of the elbow; this procedure is especially applicable when, along with the nerve lesion, there is also an ununited fracture of the humerus.

The humerus may be shortened to the extent of two inches, or even more, without serious disability. To ensure subsequent union in good position it is advisable to wire the fragments after they have been dovetailed by the step method. 
In cases of extensive nerve destruction in the forearm, complicated by non-union of one or both bones, shortening of the forearm is also to be recommended, the bones being dealt with at a preliminary operation and the nerve sutured at a subsequent date. Even in cases where the nerves of the forearm have been extensively destroyed it will be found that an end-to-end approximation of the stumps can almost invariably be effected if the procedures recommended in this article be adopted. The writer has not found it necessary, therefore, to shorten the bones of the forearm in cases in which they have been uninjured. In this connexion, too, it may be mentioned that the writer, on two occasions, has been able to effect an end-to-end suture of the stumps in patients who had previously undergone a nerve-grafting operation on the supposition that a direct end-to-end approximation was impossible: the suspicion that it was possible, was aroused by the comparatively short incision which had been employed at the original operation.

\section{TREATMENT OF NERVE INJURIES WHERE PAIN IS THE CHIEF DISABILITY}

In certain cases the motor function of a nerve may be little interfered with, and anaesthesia may be slight, yet the limb may be rendered useless by pain referred to the distribution of some particular nerve, and often associated with tenderness in the skin and muscles supplied by that nerve. All grades are found, the most extreme form being that originally described by Weir Mitchell under the name of 'Causalgia', from the burning character of the pain, which is somewhat relieved by cold wet applications.

In treating these cases, it is important to find out how much of the symptoms are due to a psychic element, which naturally will not yield to any peripheral operation, and how much to some localized lesion in the nerve. Many of the less extreme forms yield to neurolysis. The severe ones which were encountered by the writers were cured by division and suture of the nerve in a healthy part above the lesion. The pain had been such that the patient was willing to put up with the paralysis and risk the future failure of regeneration, the matter having been fully explained to him. In cases where an ascending neuritis has passed up as high as the spinal roots, the only treatment likely to relieve the pain is resection of the corresponding posterior nerve roots; fortunately such cases are rare. Alcohol injections into the offending nerve have been recommended by some surgeons as being less radical than a resection, and in certain fortunate cases the alcohol affects the sensory fibres without paralysing the motor ones, but this is by no means constant, and in the writers' opinion the ultimate prognosis for the nerve is more precarious after injection than after resection, while in the former the relief of pain is apt to be very transitory. 


\title{
PART III \\ SURGERY OF THE PERIPHERAL NERVES
}

\author{
TENDON-TRANSPLANTATIONS
}

I. INDICATIONS.

2. Essentials for a Successful Operation.

3. Details of some Special Operations.

(I) For Musculo-spiral Paralysis.

(2) For Complete Flexor Paralysis in the Upper Limb.

(3) For Paralysis of the Intrinsic Muscles of the Hand.

(4) For Partial Flexor and Fxtensor Paralysis in the Upper Limb.

(5) For Quadriceps Paralysis and Injury.

(6) For Paralysis of the Tibialis Anticus, or a Single Extensor.

(7) For Complete External Popliteal Paralysis.

\section{INDICATIONS FOR TENDON-TRANSPLANTATIONS}

Many operations on the peripheral nerves require to be supplemented by a tendon-transplant, and in the case of irreparable injury to a nerve like the musculo-spiral, which is almost purely motor, its function can be satisfactorily substituted by a tendon-transplant. In operating for a peripheral nerve injury, it is always important to examine the condition of the muscles in the neighbourhood of the lesion, as frequently they are found cut or adherent, so that the repair of the nerve alone would not restore their function. For example, in lesions of the ulnar nerve in the forearm it is common to find the inner two profundus tendons cut, or the corresponding part of the muscle-belly destroyed. Their function can be restored in the latter case by suturing them to the corresponding sublimis tendons. The indications, then, for tendon-transplants are:

(a) Destruction of the belly of a muscle.

(b) Irreparable damage to a motor nerve:

(I) where the fibres actually enter the muscle, a not uncommon finding, and owing to the small size of the fibres, one not amenable to nerve-suture.

(2) Where all methods fail to secure approximation of the ends, after extensive destruction of the whole nerve.

(3) Where motor branches have had to be sacrificed in order to suture sensory ones, such as those of the median in the forearm, or more important motor ones, such as the ulnar 
supply to the hand, to secure which it is sometimes necessary to sacrifice the twigs to the inner half of the flexor profundus digitorum.

(c) Incomplete destruction, or incomplete recovery of a mixed nerve in cases where it is not advisable to sacrifice the part of the nerve which is intact on the mere chance of recovery of certain motor fibres.

An example of this state of affairs is the claw-hand following a partial lesion of the median and ulnar nerves in the axilla, or occurring as the result of arrested recovery after suture of the two nerves. These cases present the most difficult problem, because in them sensation may be almost normal, and all the forearm muscles acting vigorously, yet the function of the hand nil, from loss of the intrinsic muscles. In such a case, nerve suture, which entails the immediate loss of sensation and of the forearm muscles, cannot guarantee a complete recovery in the end; indeed the chances are that sensation will be less satisfactory, yet it is of vital importance in the index finger and thumb. In such a case, a form of tendon-transplant, to be described later, is advised; for though it is not likely to replace absolutely the lost lumbricals and interossei, yet it restores some very useful function to the digits, which otherwise have none.

\section{THE ESSENTIALS FOR A SUCCESSFUL TENDON-TRANSPLANTATION}

The possible varieties of tendon-transplant are numerous, and it is not proposed to attempt to describe them all in detail, for if certain general principles are observed, the actual technique will present no difficulty to the general surgeon, while the method is best modified to suit the individual case.

Certain general principles are essential for success and must be most carefully observed. Neglect of them has led to the failures which can be seen in most of the clinics for war injuries, for though the operation may have been done by a good surgeon, if he did not have special experience of these cases, he was apt to overlook some simple points which make all the difference between success and failure. These fundamental principles will now be considered.

(a) Preliminary cure of all 'static deformities'. No transplanted muscle, however powerful in itself, will overcome a contracture, or even a spasm of opposing muscles, or the shortening of ligaments. Many surgeons still fail to appreciate this fact and will not give the time for preliminary stretching of adhesions, before they attempt their tendontransplant. Yet it is absolutely essential that the joints move with perfect freedom in the direction and to the full extent to which the transplanted muscle is to be asked to take them. In a musculo-spiral paralysis, if there is a contracture of the wrist in flexion, no muscle will 
give active dorsiflexion, until this has been attained passively. Similarly with the claw-hand, it is useless to try to replace the lumbricals until the spasm, or shortening of the long finger flexors has been overcome.

In the writer's opinion, by far the best method of overcoming these contractures, whether due to spasm or actual muscle-shortening, is by persistent, moderate traction, begun in the line of the deformity, the direction being altered as the relaxation of the shortened structures allows. The advantage of the method is that it throws no sudden strain on scar tissue and so avoids the acute inflammatory reaction by which that tissue otherwise responds; secondly it prevents any grinding of joint surfaces together, which also is apt to produce inflammation. The actual method of application of the traction is unimportant. For the fingers the 'banjo-splint' of springy metal, which was employed at Bangour, is useful, if the wrist and radio-ulnar joints are free; if these are fixed, it is difficult to apply the splint. Equally effective is a plastercast in which is incorporated a wire 'out-rigger' to hold the traction strings. These are best applied as loops of fine rubber-tubing, fixed to the splint by a small brass-hook and to the patient's fingers by a spica of adhesive strapping. The cast should be valved, so that massage and even baths can be applied daily. In fixing the wire out-rigger to the cast, it should be arranged so that it will not interfere with the valving of the cast and will remain firmly attached to one half. In forearm casts this is most conveniently the flexor aspect. In applying traction to the fingers, it is essential to fix the wrist and elbow, otherwise the splint will merely be pulled off the hand.

(b) The second essential for a successful operation is that the active muscle which is to be made to do a new work, should be a vigorous one and equal to the task demanded of it. It is not always possible to obtain a new muscle equal to the paralysed one, and every healthy muscle has a certain power of hypertrophy in response to increased demands on it; nevertheless it must be remembered that every muscle has an inherent limit to its capacity even to hypertrophy, so that some muscles are incapable of replacing strong ones. Thus the palmaris longus acts well as an extensor of any of the joints of the thumb, and gradually hypertrophies; whereas in one case where it was transplanted into the extensor communis digitorum, it proved unequal to the task.

Occasionally one meets a case where the whole brachial plexus has been injured, but spontaneous recovery has occurred in all parts except the musculo-spiral muscles. In such a case, it is sound to transplant the flexors to replace the extensors, even if the former are not back to their normal strength. Two such cases were done with success at Bangour.

(c) The active, transplanted muscle must be made to pull in as 
direct a line as possible, care being taken that no angulation occurs where it passes from one fascial compartment to the other, as any kinking impairs its force.

(d) Correct tension is one of the most important factors in the success of these operations. Experience has shown that the maximum effect of the transplanted muscle on the joint on which it acts, will be that attained at the operation, i.e. if the wrist-joint is held dorsiflexed to $45^{\circ}$ while a muscle is being sutured taut into the wrist extensors, then $45^{\circ}$ is all the dorsiflexion that will ever be attained by the transplanted muscle. It is essential, therefore, that all the joints over which the muscle passes should be put in the optimum position; then the distal end of the passive (paralysed) tendon must be pulled very taut, to allow of no slack there, while the active tendon must also be pulled moderately

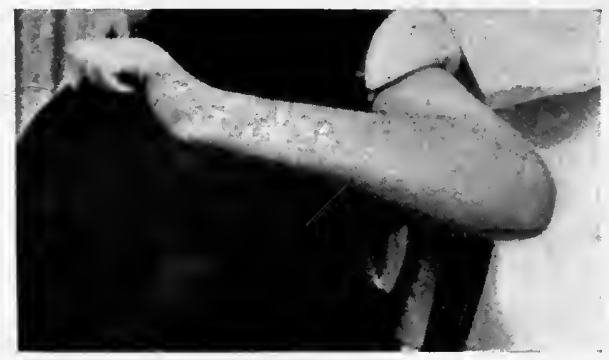

Frg. 48.-Extension of wrist by transplanted pronator teres, which had only recently recovered from injury to brachial plexus (whole limb completely paralysed at first-musculo-spiral failed to recover).

taut. It must be remembered that the active muscle which is being transplanted is an elastic structure and has a normal tonicity, which can be overcome by violence, that may result in an overstretch paralysis, or at any rate paresis, very detrimental to the operation. This question of tension is therefore a very delicate and very vital one. It may be said that if the joints are placed in the relaxed position for the muscle's new action, are held carefully by the assistant from the moment of stitching till the end of the operation, and are then fixed by careful splinting so that gravity, etc., cannot strain the muscle after the operation; then a strong pull on the paralysed (distal) tendon, and a moderate one on the active (proximal) one will give a satisfactory result. From the above, it will be seen that continuous support of the joint for some time after the operation is very important in preventing later over-stretch paralysis of the transplanted muscle.

(e) As regards the bed in which the transplanted tendon is to lie, it has been found that the subcutaneous fat is by far the best. Although light adhesions probably form after every operation, yet if the trans- 
planted muscle is a strong one, it will easily pull itself free. Judicious after-treatment, which will be referred to immediately, plays an important part in this connexion.

( $f$ ) As regards suture material, it is important to have a strong substance, which will not break, nor absorb until the two tendons have become united by firm fibrous tissue, not the young scar-tissue which may stretch. For this reason the writers have been in the habit of using linen-thread, no. 60 ; or even stronger for leg tendons.

(g) The question of a synergic connexion between the active and passive muscles is an important one, but the ideal is not always possible of attainment. As Beevor pointed out in his Croonian lectures, certain groups of muscles are always thrown into action together by a reflex mechanism, quite independent of the patient's volition, and indeed usually without his consciousness of the fact. For example, voluntary contraction of the extensor communis digitorum is always accompanied by involuntary, 'synergic' contraction of the wrist flexors. This contraction of the wrist flexors is not abolished by paralysis of the extensor communis, and to the patient's surprise, when he tries to extend his knuckles, his wrist falls into flexion, as the flexors are now unopposed. If these same wrist-flexors are transplanted by the surgeon into the extensor communis, then they they will inevitably contract when the patient wills to straighten his fingers and they will now carry out that action, i.e. practically no education of the transplant is required. It is important therefore for the surgeon to study what groups of muscles are synergic, and whenever possible to use corresponding groups. Unfortunately, many instances occur when such are not available. Some writers have laid down the principle that under no circumstances should a true antagonist be used for transplantation into its opposer. It is possible that the antagonist can never be trained to work independently of its old group in support of its new one; nevertheless, functionally the result of the operation may be good; for instance, the very fact that the transplanted muscle contracts simultaneously with its old group in a position antagonistic to them will prevent the unbalanced action of these and so restore stability and balance to the limb. The writer saw striking instances of this when one of the hamstrings had been transplanted into the quadriceps; before the operation the men were unable to support themselves on the limb, because the unopposed flexors caused it to give way in flexion; after the operation they were able to keep the limb actively extended, and after much practice to flex it also to any desired angle. From this it is evident that synergism should not be made a fetish. Moreover, it must be borne in mind that muscles frequently contract reflexly with their antagonists in order to prevent sudden unbalanced action, and are continually adjusting their length to altered 
positions of the joints, so that there is no such thing as a simple, pure antagonist. Indeed, the whole subject is a most complex one, and is worth much careful study.

(h) After-treatment plays a more important part in connexion with tendon-transplants than with almost any other operation in surgery.

It has three aims :

(I) To prevent over-stretching of the transplanted muscles in the early stages, lest the stitches cut out, or the muscles be paralysed by strain.

(2) To prevent or break down adhesions by the judicious use of movement, by massage, and by baths.

(3) To educate the patient in the use of the muscles in their new positions.

As regards the prevention of over-stretching : a splint must be applied at the end of the operation to keep the limb in exactly the position in which it has been held by the assistant. It is important to select the splint and fit it to the patient before the operation, seeing what an important factor towards success it is. In discarding or modifying the splint, the best principle to follow is that laid down by Sir Robert Jones for recovering muscles in infantile paralysis, i.e. to allow just as much strain on the muscle as it is capable of resisting, and no more. Thus when a certain degree of dorsiflexion of the wrist is obtained voluntarily, then lower the splint a little; but if the action is weaker the next day, it is evident that strain has been thrown on the muscle too early, and the support must be restored. Once the patient has acquired control over the transplanted muscles, their strength increases remarkably from day to day. The splint should be omitted for an hour one day, and if this does not prove injurious, for increasing periods. Many patients realize themselves that it is beneficial to continue wearing the splint at night even after the function of the hand has become good and they have ceased to wear it by day. Thus no definite rule of so many days or weeks can be laid down ; each case must be judged on its merits, and the function test is the only safe one.

As regards the prevention and treatment of adhesions, hot baths, whether of the whirlpool type, or merely a hot compress, seem valuable; but if they are begun before the wound is absolutely firmly healed, they are apt to break it open, and so interfere with massage and exercises, thereby doing more harm than good.

The same remarks apply to massage, which is valuable, so long as it does not disturb the wound. As unusually long incisions are necessary; and as any bursting of the wound with subsequent adhesion to underlying tendons impairs the functional result, the healing of the skin wound plays a specially important part in these cases. 
Adhesions are best prevented by early voluntary use of the transplanted muscles, followed later by judicious passive stretching, so that the tendon is then freed in both directions. It is essential that the surgeon should inspect these cases once a week at least, preferably twice, and that they should be treated by a masseuse with special experience of them and one who works under the direct supervision of the surgeon. It is desirable that the masseuse should be present at the operation, though unfortunately this is not always practicable. She should certainly have seen at least one operation of the same type, or she will never actually realize what she is working with, and for what she should strive.

Re-education of the patient and his muscles is naturally of prime importance. Some patients learn the new actions very rapidly, just as some people learn games much faster than others, and this is especially the case where synergic muscles have been used. With a patient of dull intellect or deficient muscle-sense the process of education may be a very tedious one, but the most hopeless-looking individual can be taught by a patient instructor.

The simplest method to adopt is to ask the patient to perform the action which was formerly carried out by the transplanted muscle; then show him the tendon acting in its new course, and induce him to continually repeat the voluntary effort while observing the tendon. With practice he will acquire the knack of deliberately performing the new movement by means of the transplanted muscle. The energetic patient will soon develop strength in the movement, whereas the inert individual, who only exercises when under supervision, will make slow progress.

Electrical treatment is of value in these cases as a means of demonstrating to the patient the new action of the transplanted muscles, and also to free adhesions by more frequent movements of the muscles than are at first obtainable voluntarily owing to the intervention of fatigue. It is well known that fatigue is always manifested in the nerve centres, before the peripheral nerve, much less the muscle itself, ceases to respond to electrical stimulation; this phenomenon is especially marked in transplanted or recovering muscles. Some of them show early fatigue with the faradic current, in which case it is best to use galvanism. Otherwise it is immaterial which current is used. Whichever gives the most vigorous response should be employed. It is often remarkable the way in which one demonstration of the muscle's action by electricity enables the patient to regain voluntary control of it. In order to counteract adhesions, it is necessary that the contraction of the muscle should be vigorous and jerky, so that no mere hardening of its belly suffices, a point which it is sometimes difficult to impress on the masseuse. 


\section{TYPICAL TENDON-TRANSPLANTATION OPERATIONS}

(I) For Musculo-spiral Paralysis (forearm muscles).

This operation has now become classical, and is one of the most successful forms of tendon-transplantation. Though all operators observe the same principle in carrying it out, yet there are considerable variations possible in the technique. The method in use at Bangour will be described now. Following the lines laid down by one of the authors (H. J. S.), long incisions were used and free dissection of the muscles,

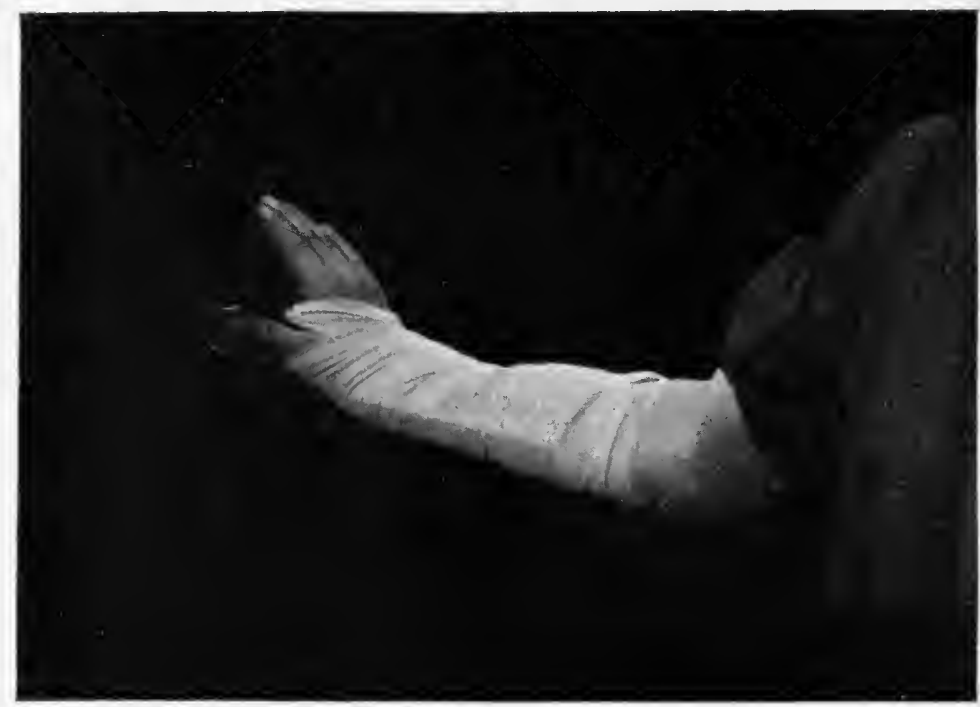

FIG. 49.- - Long cock-up splint for use after tendon-transplant or in musculospiral paralysis. Note the position of thumb in extreme extension at all joints, and the knuckles which are barely fully extended, to avoid stiffness.

and the active tendon was threaded through a slit in the paralysed one and then sutured with ' 60 ' linen thread. The muscles used were :

Pronator radii teres into extensores carpi radiales longior and brevior (to give dorsiflexion of the wrist).

Flexor carpi radialis into extensores ossis metacarpi and brevis pollicis (to restore extension of the first two joints of thumb).

Palmaris longus, when present, into extensor longus pollicis (to extend terminal phalanx of thumb). This muscle was found absent both sides in I7 per cent. of normal men tested, and absent on one side in another 8 per cent. ( 25 per cent. in all).

Flexor carpi ulnaris :

(a) The tendon into extensor communis digitorum, extensores indicis and minimi digiti, and into the extensor longus pollicis when the 
palmaris longus was absent (to restore extension of all the knuckles). It is important to note that these extensors do not normally extend the interphalangeal joints, being unable to overcome the tone of the long flexors; when the latter are paralysed, the extensor communis is able to extend the middle and terminal phalanges, but as the flexors recover, the extensor can be observed gradually losing this power. The lumbricals and interossei extend the interphalangeal joints by their pull on the extensor communis tendons.

As the communis tendons pass over the interphalangeal joints, they are a iittle stretched by flexion of the joints, and therefore to attain the correct tension in tendon-transplants into them, it is best to keep the fingers completely extended, and not merely at the knuckles. It is equally important to see that the knuckles are not hyperextended by the transplant, because any stiffness of this type is most difficult to overcome and is most disabling; the function of the hand never requires extreme extension at the knuckles.

(b) The lower fleshy fibres into extensor carpi ulnaris, so as to counteract the tendency to radial deviation of the hand, which occurs from the pull of the pronator teres on the radial extensors. The tendon of flexor carpi ulnaris, after being dissected up from these fleshy fibres, is made to pass to the back of the forearm superficial to them; it is important to see that the tendon is freed sufficiently high, so that it is not in any way anchored by the other fibres. As the nerve fibres enter the belly immediately below the elbow, there is no fear of paralysing the muscle by this free dissection.

\section{Details of technique.}

(a) Dorsal incision to prepare the paralysed tendons for the transplant. The line of this incision is from the external condyle of the humerus to the tubercle on the back of the lower end of the radius. The incision begins in the middle of the forearm, just below which the insertion of pronator teres will be exposed, and it ends at the level of the wrist joint.

In the upper part of the wound there is exposed the interval between the bellies of the extensor communis and the extensor carpi radialis brevior; this is opened up and the radius exposed where the tendon of the pronator teres is wrapped round it and inserted into it. Supination of the forearm brings the tendon well into the field. It should be detached with a periosteal elevator as the tendon is flat and papery, so that care is required to obtain it long enough and strong enough for suture. It must be remembered that its attachment to the radius is below the middle of the forearm, sometimes extending very low, so that if it is sought for above this level, the fibres of the supinator brevis are apt to 
be mistaken for it. When the tendon has been detached, it is pulled on and the belly freed by blunt dissection from the flexor carpi radialis, with which it is intimately associated. In doing this care must be taken not to injure the radial vessels where they cross the tendon; there is sometimes troublesome bleeding from muscular branches. At the level at which the pronator has been freed, the deep fascia over the extensores carpi radiales is opened and the two muscles are pulled on to see that they run freely to their insertion. These paralysed muscles have nearly always adhesions to the tendon sheathes, which can readily be broken by firm pulling at the operation, but not so readily by any transplanted tendon, so that the success of the operation largely depends on attention to this detail.

The pronator is not sutured to the carpal extensors at this stage, because the dissection of the flexors on the front of the forearm is made difficult by dorsiflexion of the wrist, which is essential after the suture is done; it is therefore simplest to finish as much of the dissecting and skin suturing as possible, before suturing the tendons, thereby reducing the strain on the assistant and also the risk of any accident.

In the lower part of the dorsal wound, the tendon sheath is opened just above the annular ligament and each tendon of the extensor communis is isolated and made to pull freely to its insertion; then they are all retracted inwards, so as to pick up the extensor longus pollicis, which lies deep to their radial border; on its ulnar aspect the extensor indicis is also picked up and both are freed. These two muscles are apt to be fleshy, even down at this level. The extensor communis is next retracted outward, and the extensor minimi digiti found deep to its ulnar border, also often fleshy. If the transplant is merely made into the common extensor tendons of the index and little fingers, good extension of those digits is not usually obtained, so that it is important to find and use the special extensors also. This was shown in a case where the extensor indicis had lost its connexion after the operation, and although the extensor communis was being well acted on, yet during extension the index finger lagged behind the others. The sheath of the extensor carpi ulnaris is opened at the same level. The skin edges on the dorsum are now held together with Lane's clips, while incisions are made on the front of the forearm; if this is not done the elasticity of the skin is lost and it is difficult to make a clean incision.

(b) An incision is made along the ulnar border of the flexor carpi ulnaris tendon, which is dissected free from fleshy fibres up to the middle of the forearm and cut across opposite the wrist joint. Care is taken not to open the deep fascia over the flexor profundus digitorum, which protects the ulnar nerve and vessels, where they run under cover of the flexor carpi ulnaris. The fleshy part of the ulnaris is also freed from 


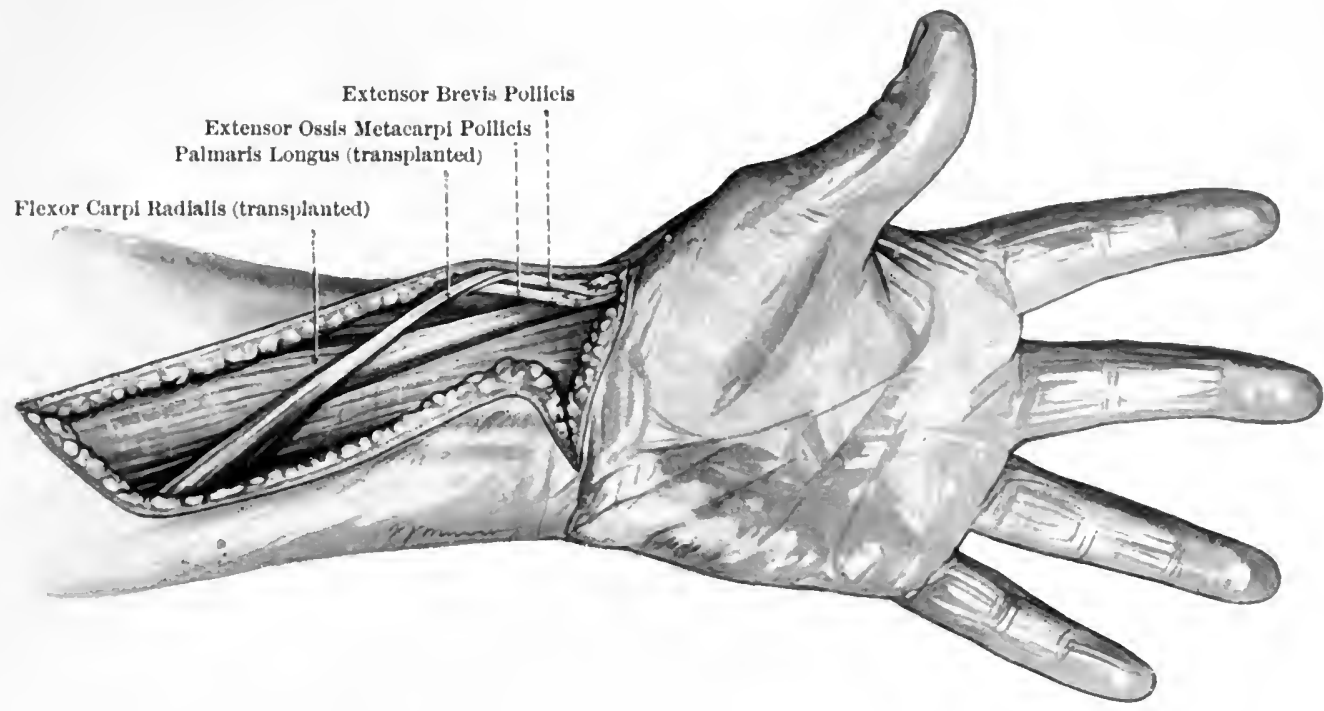

Fig. 50a.-Tendon-transplantation for Musculo-spiral paralysis-Flexor Carpi Radialis transplanted into short extensors of thumb; Palmaris Longus passing to dorsum of limb to reach Extensor Longus Pollicis.

Flexor Carpl CInaris tendon detaehel from lelly

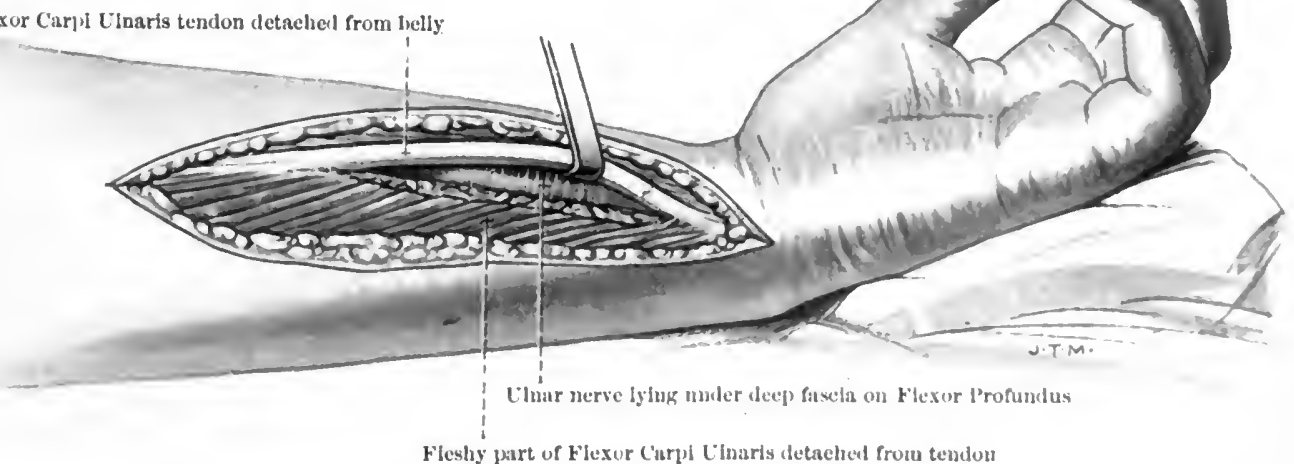

Fleshy part of Flexur c'urpl Cinarls detached from teindon

FIG. 50 b.-Flexor Carpi Ulnaris tendon freed from its bed and from the fleshy fibres preparatory to passing it to dorsum of limb. Note the Ulnar nerve lying under a layer of deep fascia on the surface of Flexor l'rofundus Digitorum. 


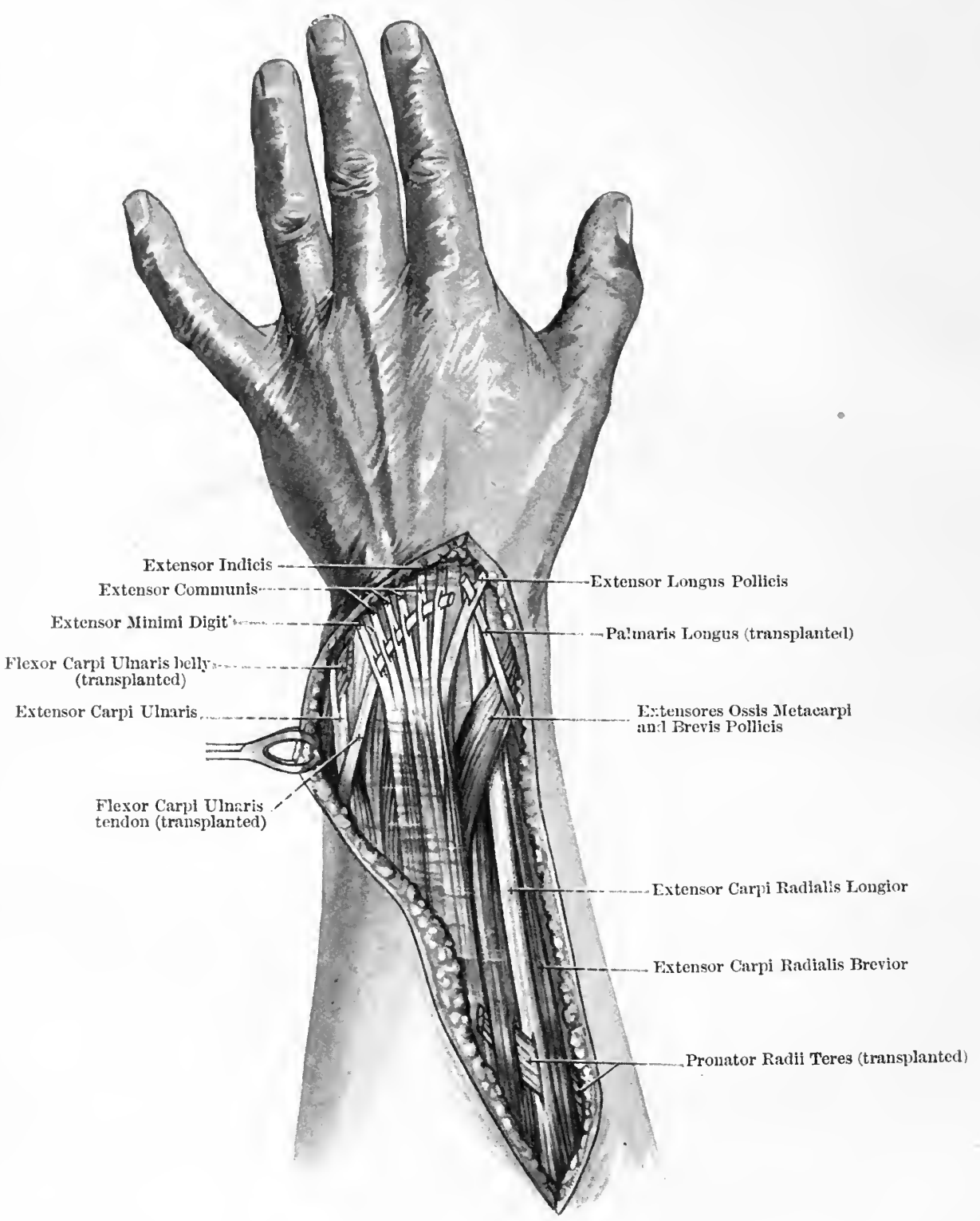

FIG. 50c.-Dorsum of forearm showing transplants of: Pronator Radii Teres into Radial carpal extensors; Flexor Carpi Ulnaris belly into Extensor Carpi Ulnaris; Flexor Carpi Ulnaris tendon into extensors of all knuckles; Palmaris Longus into Extensor Longus Pollicis. For the sake of simplicity, the tendons are not shown drawn taut with the wrist dorsiflexed, as they must be before any sutures are inserted. 
the ulna and its insertion, so that it can be pulled to the back of the forearm. The skin is next undermined between this wound and the dorsal one, so as to afford passage for the tendons; if a blunt dissector is passed close to the deep fascia and the ulna, a space is easily opened up without damage to vessels, but if an attempt is made to pass immediately under the skin, there is apt to be troublesome bleeding from the ulnar veins. The nearer the second incision is made to the ulnar border of the forearm, the less skin will there be to undermine and the more easily can any injured vessels be ligatured; while the flexor carpi ulnaris is just as easily reached.

When the flexor carpi ulnaris has been passed to the back of the forearm and clipped with Kocher's forceps to prevent it slipping forward again, the front wound can be sutured.

(c) An incision is next made along the radial border of the flexor carpi radialis tendon in the lower two-thirds of the forearm. The tendon is isolated, divided opposite the wrist joint, and then freed up to the level of its fleshy belly, care being taken not to interfere with nerves or vessels entering it.

If a palmaris longus is present, it is similarly freed and detached. Care must be taken not to mistake for it the middle-finger tendon of the sublimis, which also lies along the ulnar side of the flexor carpi radialis, but which is covered by the deep fascia, whereas the palmaris longus is the only muscle which runs in the superficial fascia.

The lower end of the skin incision is curved outwards towards the root of the thumb, and then the tendons of the extensor ossis metacarpi and brevis pollicis are easily exposed opposite the wrist joint. Their sheath is opened and they are pulled free, so that the flexor carpi radialis may be transplanted into them; they are split for $\frac{1}{2} \mathrm{in}$. by a tenotome and the flexor tendon threaded through them and both pulled taut before suturing. Care must be taken not to miss the extensor brevis as the ossis itself is usually in two or more portions, and so may be mistaken for the second muscle. From this moment to the end of the operation the thumb must be held fully extended at its two proximal joints.

The second anterior wound can now be sutured. If a palmaris longus is being used, a tunnel must first be made for it to the back of the forearm round the radial border of the limb. If the anterior wounds are not closed at this stage, it is very difficult to get approximation of the skin after the wrist and fingers have been fixed in extension. During dissection on the flexor aspect of the limb, it is convenient to have the wrist slightly flexed, either by the assistant, or by a sandbag.

(d) The dorsal aspect of the limb is again dealt with, the pronator and flexor tendons being threaded through the extensors and sutured to under correct tension, in the manner described under 'General principles'. 
Finally the dorsal wound is sutured; ample dressings are applied, as there is always considerable oozing and it is very undesirable to disturb the position of the parts to apply fresh dressings for several days. A suitable splint is applied and the patient kept quiet with morphine. There is considerable pain for about 36 hours after these operations, after which it subsides, provided the dressings are not too tight. If the precaution has been taken of putting on the inner dressings loose, and only the outer bandage firm, the latter can be cut at the end of 24 hours and a fresh one applied over it without disturbing the splint. The tips of the patient's fingers should be left exposed, so that the condition of the circulation in the limb can be watched.

The question of after-treatment has been sufficiently dealt with above.

\section{(2) For Complete Flexor Paralysis.}

In cases of paralysis of all the flexors of the digits, extensors can be transplanted to give satisfactory flexion, but unfortunately these cases are nearly always complicated by extensive anæsthesia, which is in itself most disabling, and also by paralysis of the intrinsic muscles of the hand, without which the flexors are useless from the point of view of function. The question of substitution for the intrinsic muscles will be dealt with in a later section. With regard to the flexors, the following has been found a satisfactory transplant:

Extensor ossis metacarpi pollicis, or part of it, into flexor longus pollicis (to flex terminal joint of thumb and give it stability).

Extensor carpi radialis longior, which has a long tendon, split in two parts to both flexors of the index and middle fingers respectively. It appears better to fix the two tendons to the same finger together, rather than the two sublimis tendons, because the sublimis and profundus to any one digit practically always act simultaneonsly. The extensors of the carpus are synergic with the flexors of the fingers and thereby well adapted for transplantation.

Supinator longus (brachio-radialis), also split, for the flexors of the ring and little fingers.

In carrying out this transplant, the tension of the muscles should be exactly adjusted to the function which it is intended that the digits should perform. One cannot expect to reproduce a normal hand, but one may benefit the patient greatly, if one restores to him the pincer action of the index finger and thumb, together with a grip of the inner two or three digits which will enable him to carry a bag. To attain this aim, at the operation the tendons should be sutured with the thumb fully flexed at its terminal joint, with the index finger flexed sufficiently to meet the end of the thumb, with the inner two digits flexed almost into the palm, while the middle finger is somewhat less flexed, for fear that a contracture might interfere with the function of the index and 
thumb. As the functional position of the limb involves slight dorsiflexion of the wrist, and as one of the powerful dorsiflexors is being reversed in its action, it is undesirable to flex the wrist while securing flexion of the digits. The wrist joint must be fixed by the splint which secures the fingers, otherwise the transplanted muscles may be subjected to strain by its movements. The best method of fixation after the

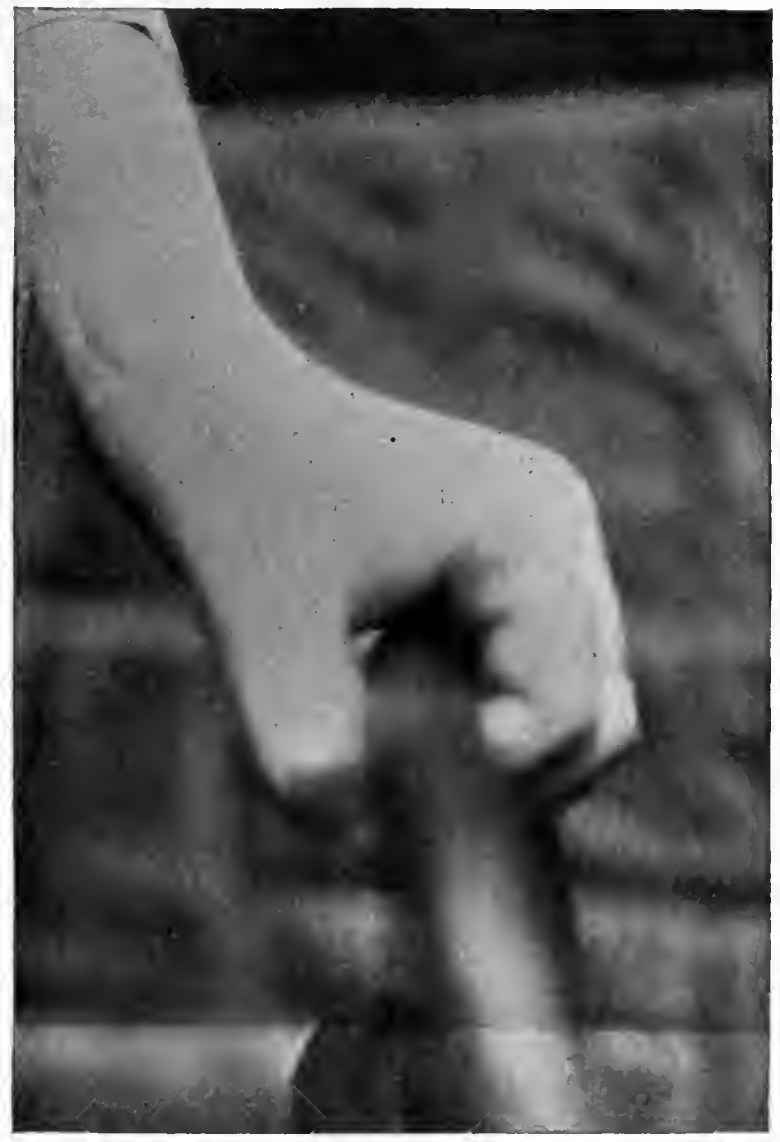

FIG. 51.-Lifting a chair-a case of complete flexor paralysis treated by transplantation of the extensors.

operation is to bandage the fingers and thumb into a fist over some wool and then to apply a short cock-up splint, which keeps the wrist dorsiflexed and prevents the bandage from slipping over the fingers.

\section{Details of Technique.}

(a) Dorsal incision to expose the extensors ready for transplantation. This incision should run along the radial border of the forearm 


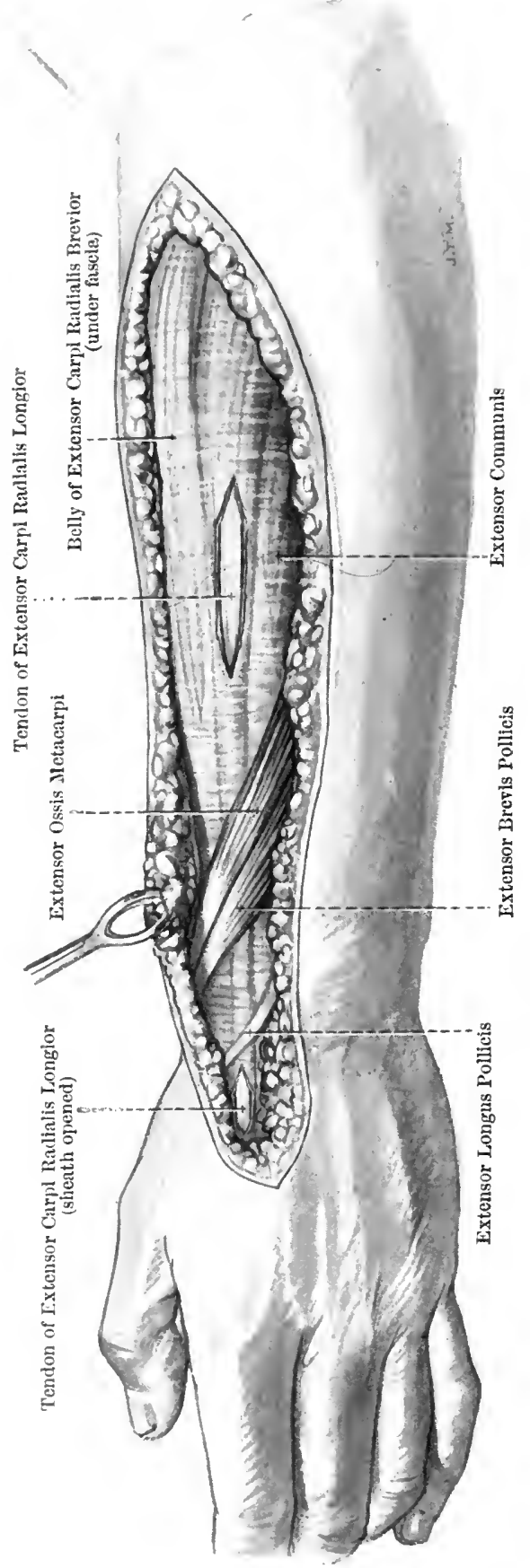

FrG. $52 a$.-Dorsum of forearm showing extensors exposed preliminary to transplantation into the paralysed flexors.

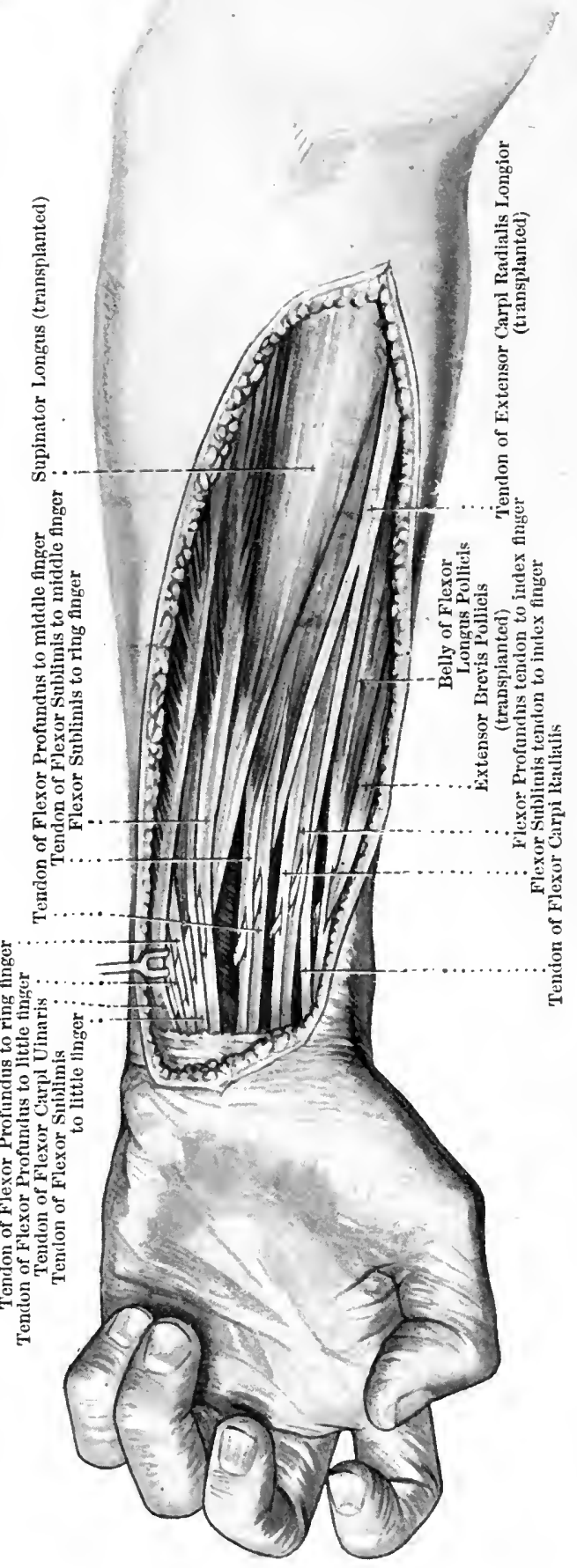

FIG. 52b. - Volar aspect showing transplants done, i.e. Extensor Ossis Metacarpi Pollicis into Flexor Longus Pollicis. Extensor Carpi Radialis Longior into flexors of index and middle fingers. Supinator Longus into flexors of inner two fingers. 
between the supinator longus and extensores carpi radiales, and should extend from the root of the thumb up to the upper third of the forearm.

In the lower end of the wound, the extensor ossis metacarpi pollicis is found crossing the carpal extensors; it is freed down to its insertion and divided as low as possible.

Below the extensor ossis, the tendon of the extensor longus pollicis is also found crossing the carpal extensors, i. e. below the annular ligament ; it is retracted upward without opening its sheath, and below it an incision is made into the sheath of the extensor carpi radialis longior close to its insertion into the second metacarpal, and the tendon cut across.

Next the inner edge of the wound is well retracted and the supinator longus detached from its insertion, the muscle then being freed as far as the upper end of the wound, great care being taken to avoid injury to the radial vessels and nerve. The extensor carpi radialis brevior (fleshy) lies immediately on the radial border of the supinator longus, with the strap-like tendon of the extensor carpi radialis longior coursing down its surface; it is the latter which we require. A small longitudinal incision is made through the deep fascia to expose this tendon in the upper third of the forearm, and as it has already been detached from its insertion, it can now be pulled right out of its bed into the upper part of the wound, while the fascia over the brevior is undisturbed, except for the small incision.

(b) An incision is next made on the front of the forearm along the line of the flexor carpi radialis, to expose the flexors of the digits.

The flexor carpi radialis is first retracted inwards, so as to expose the flexor longus pollicis, which lies on the lower end of the radius just under cover of the radial border of the carpal flexor. The tendon is isolated immediately above the annular ligament and pulled free of any adhesions to the sheath.

Next the flexor carpi radialis is retracted outwards, and the sublimis and profundus tendons to the fingers are isolated above the annular ligament and identified. Their arrangement is well known : the sublimis in pairs, those to the middle and ring fingers on the surface; deeper the ones to the index and little fingers on corresponding sides; deeper still the profundus tendons, their surface forming an oblique plane. The large solitary tendon to the index finger lies deep to the sublimis, while a broad tendon, often common to the other three fingers, extends beyond the inner border of the sublimis, the part to the little finger lying under cover of the flexor carpi ulnaris; this, or the ring-finger portion, bears the ulnar vessels and nerve on its surface. The ring and little finger tendons are often undifferentiated at the upper border of the annular 
ligamient, so that this may need to be partly divided in order to expose them satisfactorily.

The skin is now undermined on the radial border of the forearm between the two wounds in order to allow the extensors to reach the flexor aspect ; care must be taken that there is no kinking of the tendons and that the radial vessels are not injured. The transplant is finished on the lines described above under 'General principles'. It is simplest to suture the dorsal wound before fixing the tendons. If this is done, the surgeon's gloves and forceps must be well rinsed, or changed in order not to carry organisms from the skin in among the tendons.

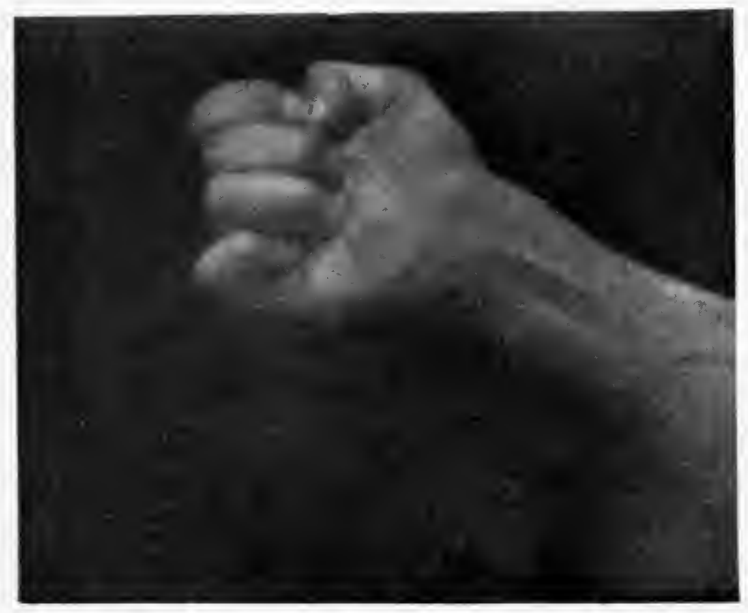

FIG. 53.-Flattening of the hand when all the thenar muscles are paralysed.

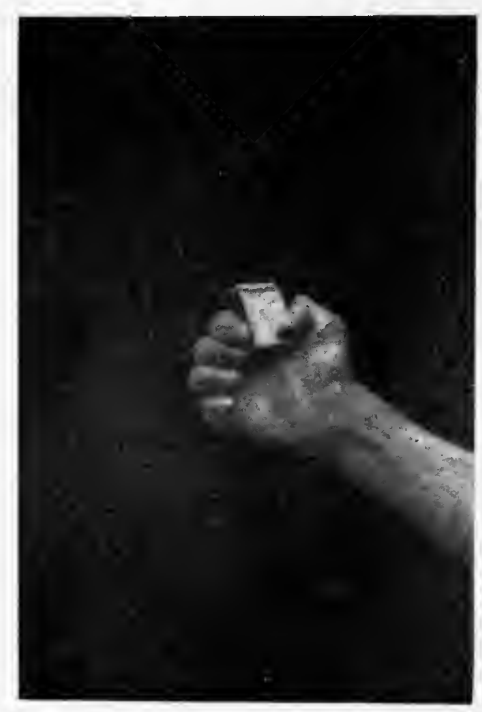

FIG. 54--Loss of the pincer action of the thumb; grip by long flexor only, aided by long extensor, which together give a pseudo-adduction.

\section{(3) Transplantation to Compensate for Loss of the Intrinsic muscles of the Hand.}

No satisfactory transplant appears to have been devised to replace the intrinsic thumb muscles, whose loss makes that digit almost useless, as it falls back into line with the other digits and is unable to be opposed to them for picking up objects. Fortunately this function can be largely restored by the operation of arthrodesis of the first carpo-metacarpal joint, as devised by Major Baldwin, U.S.A., who pointed out that the functioning position of the thumb for almost all purposes is one in which the first and second metacarpals meet at an angle of $60^{\circ}$; so that if the joint is fixed at this angle, the loss of the muscles which normally bring 
it into opposition, is scarcely noticed. This operation, however, does not restore the powerful grip of the thumb which depends on the adductor pollicis, and for which no substitute has yet been devised. The arthrodesis operation restores admirably the pincer action, which is normally employed in picking up small light objects, where delicacy rather than strength is needed. (Figs. 55 and 56.)

Clawing. The other function of the intrinsic hand muscles, whose loss induces a severe disability, is flexion of the knuckles together with extension of the interphalangeal joints, which is dependent on the action of the lumbricals and interossei. If only one of these groufs alone is paralysed, the other is usually sufficient to prevent clawing, but when both are gone, the long flexors are found to have no power to flex the knuckles, and the long extensors none to extend the interphalangeal joints. Under these circumstances, when the patient attenipts to close his hand: the fingers are curled up on themselves by the action of the long flexors and hyperextended at the knuckle by the unopposed action of the long extensors, so that they do not reach the palm and are useless for grasping. The condition is exceedingly disabling, and therefore any procedure which iniproves the function of the limb is welcome, even if it be impossible to restore the normal use.

One of the authors (H. J. S.) devised an operation to alter the insertion of one of the flexor tendons, so as to make it a flexor of the knuckles, and by suturing it to the externsor communis tendon just distal to the knuckle, to make it also an extensor of the interphalangeal joints, i. e. to make it take the course of the lumbrical.

In the operation as first devised, half of the flexor profundus digitorum tendon was transplanted, the flexor sheath being split longitudinally opposite the two proximal phalanges. Although this improved the function of the digit, yet the extensive opening of the sheath gave adhesions of the flexor tendons and a tendency for them to spring forward like bowstrings. It was found that this drawback could be avoided by utilizing half the sublimis tendon, where it splits to let the profundus through, while the tendon was able to be detached from its insertion through a small lateral slit in the sheath and passed to the back of the firger through another small slit, neither of which interfered with the function of the sheath. Finally it was found that to attain a correct balance, it was necessary to transplant both halves of the sublinis, instead of only one. This operation can naturally only be performed when both sublimis and profundus are working vigorously. It certainly enables the digits to be guided into the palm, and the index finger to meet the thumb and pick up objects. It promises, with careful reeducation, to allow of flexion of the phalanges, independent of flexion of the knuckles, because the profundus, acting on the former, has been 


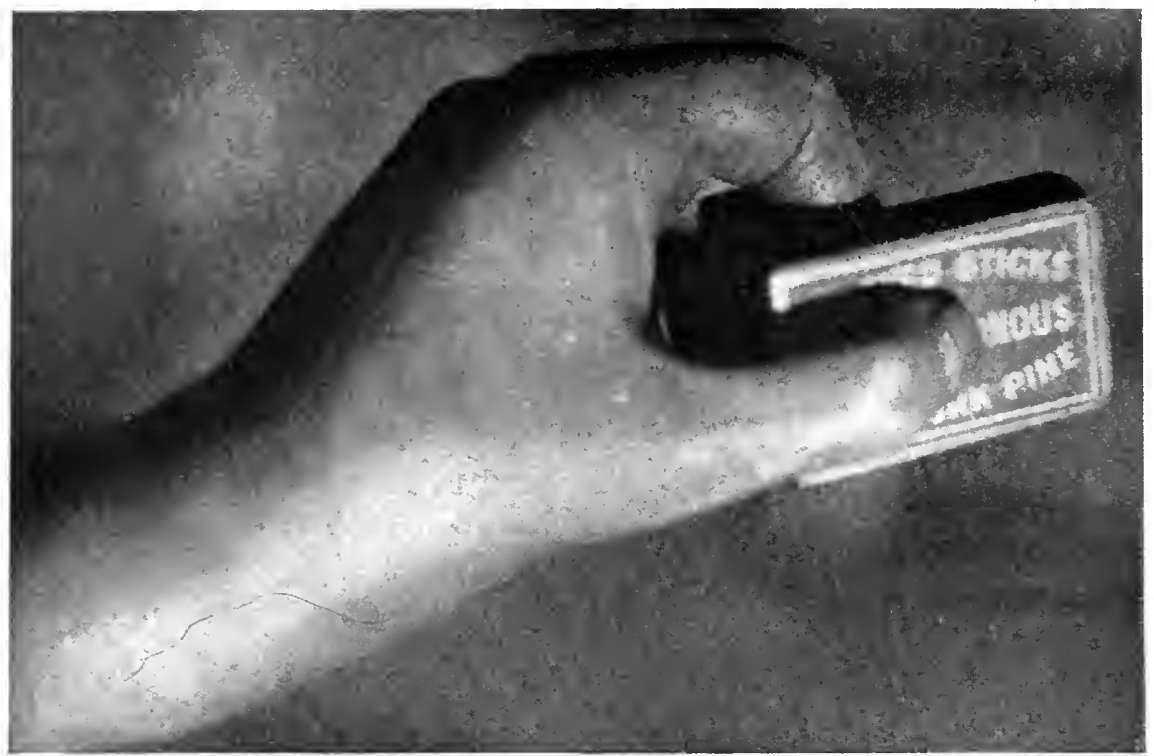

Fig. 55.-Pincer action of index finger and thumb restored in case of complete paralysis of the flexors and thenar muscles by arthrodesis of the first carpo-metacarpal joint, transplant of extensors, and finally transplant of half flexor profundus tenclon to act as lumbrical.

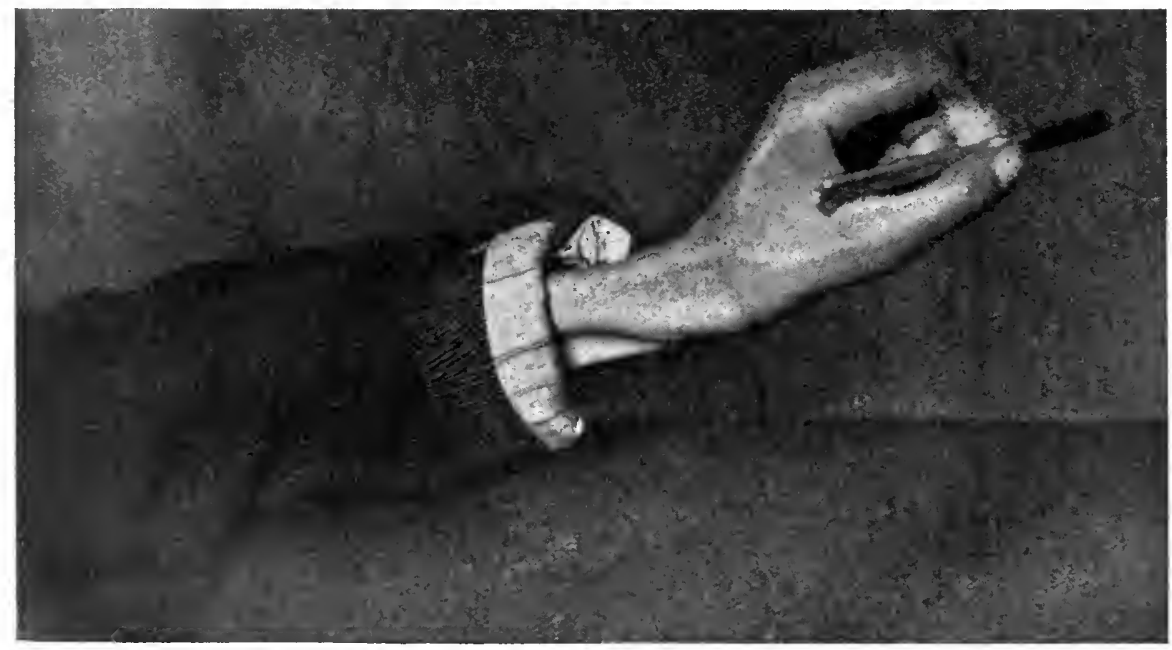

Fig. 56.--Pincer action restored to thumb and index finger in case of median and ulnar suture where the intrinsic muscles failed to recover, though the long tlexors did so. Arthrodesis of first carpo-metacarpal joint done and transplant of half hexor profundus tendon into extensor communis to act as lumbrical. 
Details of Technique. As noted often above, it is essential that any contracture, such as is apt to complicate these cases, should be thoroughly overcome before the operation is attempted.

(a) Incision on the dorsum of the affected digit opposite the middle of the proximal phalanx, to expose the common extensor tendon which widens out at this level and receives the insertions of the interossei and lumbrical into its lateral aspects, the lumbrical being on the radial side. These insertions should be defined by cleaning the edges of the tendon,

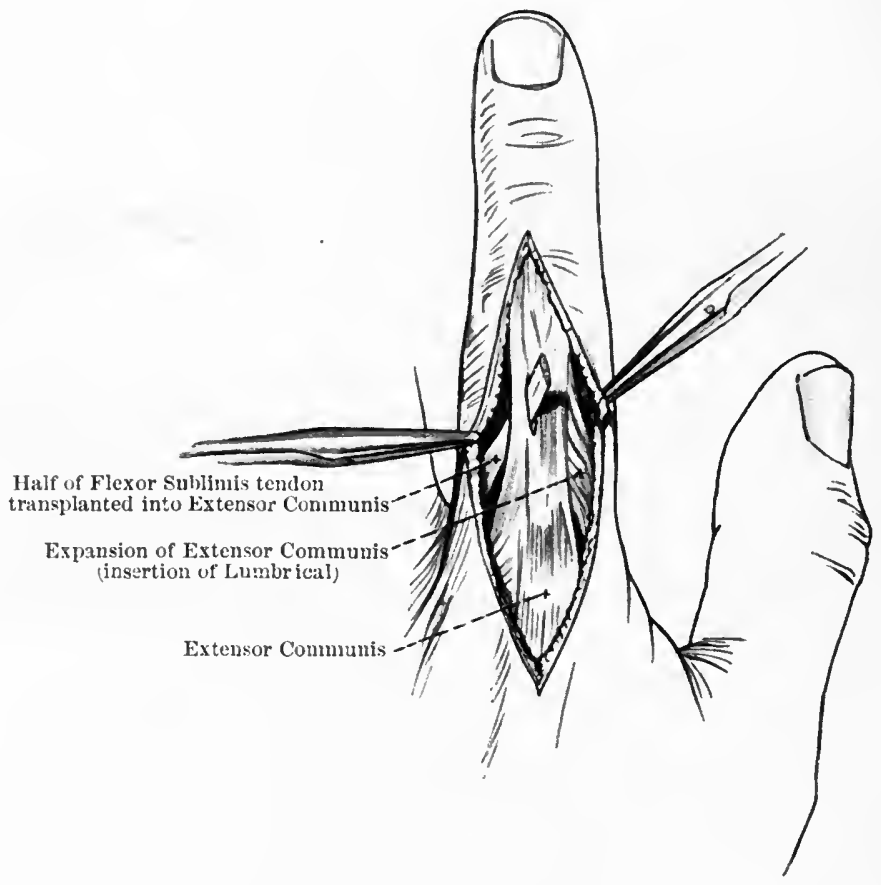

FIG. 57 b.-Dorsum of index finger, showing Ulnar half of Sublimis passed through a slit in Extensor Communis tendon before it is stitched to the latter. The extensor expansion is shown undisturbed on the Radial side, where it forms the insertion of the first Lumbrical as well as of the first Dorsal Interosseus.

and it should be raised from the phalanx, so that at a later stage it can be perforated for the passage of the transplanted tendon.

(b) Incision down the flexor aspect of the digit from the neck of the metacarpal (transverse palmar crease) to the distal interphalangeal joint. The flexor sheath should be exposed by this incision, but care taken not to open it in this line. A small lateral opening is now made on each side of the sheath opposite the distal interphalangeal joint to enable the half of the sublimis tendon on each side to be divided near its insertion. It is not essential to obtain the whole length of the tendon. 
Next the tendon is exposed opposite the metacarpo-phalangeal joint, where it has just begun to split, an opening being made on each side, so as to allow each half to be pulled out of the sheath. In making these openings in the sheath, it is important not to disturb the strong bands which hold the profundus in place opposite each phalanx, otherwise the tendon will stand forward under the skin like a bowstring, when the patient attempts to flex the finger, and will lose its pull on the terminal phalanx.

A tunnel is now made on each side from the flexor to the extensor aspect of the finger close to the bone opposite the base of the proximal phalanx, passing a little obliquely downward as it goes backward, indeed following the course of the lumbrical on the radial side. Through this the half of the sublimis on each side is brought to the back of the digit. The two portions are then inserted into the extensor communis tendon by passing them through a slit in it and suturing them with linen thread. Any superfluous portion of tendon is cut away and the raw end buried by a suture through the two sides of the communis. While the transplant is being done, it is essential to have the digit held in the position in which it is intended that the muscle shall now act, i. e. with the knuckle flexed to a right angle and the interphalangeal joints straight. Omission of this precaution will entirely spoil the result. At the end of the operation the fingers can be fixed in this position by pads of cotton wool and bandages, almost better than by a splint.

After-treatment, as with any other transplant, consists in education of the new movement, initiated by faradism usually, and later careful stretching of the transplanted muscle till full range of the finger is restored.

\section{(4) Partial Extensor and Flexor Paralyses.}

The varieties of transplant for incomplete injuries are almost as numerous as the tendons. Bearing in view the advantages of synergic action, it is evident that extensors of the thumb or digits are best repaired by a transplant of one of the wrist flexors. The flexor carpi radialis, owing to the length of its tendon, is the most suitable; the palmaris longus makes an excellent extensor of any joint of the thumb, but is too weak for all the digits at once.

Where only one carpal extensor is paralysed, it is seldom necessary to resort to a transplant, as the others hypertrophy. The extensor carpi radialis longior, with its long tendon, makes a convenient transplant into any extensor, but is probably less satisfactory from the functional point of view than a wrist flexor.

Where one, or even two flexors of the wrist are destroyed, as a rule no disability is complained of. These injuries are usually complicated 
by damage to nerves and finger tendons, which quite masks the injury to these tendons.

When the sublimis tendon to one finger is injured, the function of the digit can be restored by suturing the tendon to the corresponding profundus one. The converse holds for injuries to the profundus.

If both tendons to one digit are cut, then from the point of view of synergism, the tendon of the extensor carpi radialis longior is the best to transplant into them. In practice, it usually seems simpler to use the flexor carpi radialis, or the palmaris longus, which lie at hand without any further dissection.

To be able to flex the terminal phalanx of the digit independently of the proximal one is a luxury, and indeed an art only acquired by a few normal people, so that there is no advantage in using a separate tendon for the sublimis from the profundus. It is, however, most important to see that the tension of the two tendons corresponds, so that the transplant pulls on both equally.

In the lower limb it is stability, rather than precision of movement, which is the most important function, and therefore tendon-transplants play a less valuable part than they do in the upper limb; indeed an arthrodesis is often a more rational operation. Certain transplants, however, have proved valuable in practice and will be referred to.

\section{(5) For Quadriceps Paralysis and Injury.}

A lesion which puts out of action more than about half the fibres of the quadriceps, is most disabling, for when the patient puts weight on the limb in the flexed position, however slight the angle of the initial flexion, the limb gives way into full flexion under him ; as moreover he is unable to throw the limb straight forward in the act of walking, he cannot progress at all without support. An apparatus which holds the limb rigid, or an arthrodesis, will enable him to put weight on the limb and to walk, but has the drawback that the limb sticks out straight when he is seated, and he is unable to kneel down. In many such cases, useful function can be restored to the limb by a transplantation of one of the hamstrings into the quadriceps tendon. Although these muscles are usually considered to be direct antagonists, and as such unsuitable for transplantation into one another, yet, as has been previously pointed out, the stability of a joint often depends on simultaneous contraction of antagonists, and if this is the result of a transplant, it is functionally a good one. In practice it is found that the patient can by careful education and much practice be trained to extend the knee and also flex it to any required angle voluntarily. As regards which of the hamstrings should be used, it may be pointed out that the semitendinosus has too narrow a tendon to stand the strain, while the biceps which has a long 
strong tendon, making it in many respects suitable, yet tends to pull the patella to the outer side of the joint; yet it is support for the inner side of the joint which is most important functionally; hence the semimembranosus is likely to be the best to use. In cases where a large part of the vastus internus has escaped, the biceps makes a satisfactory transplant, if its tendon is inserted across into the inner side of the patella. It is, however, remarkable that when the vastus internus is acting, an otherwise extensive injury to the quadriceps may give very little disability. On the other hand the wasting of the vastus internus, which is an accompaniment of recurrent dislocations of the internal semilunar cartilage, causes extreme weakness of the joint, even after the offending cartilage has been removed. This teaches us that it is the inner part of the quadriceps which we must on all accounts reinforce by our transplant.

\section{Details of the Operation.}

Incision. A long $J$-shaped incision on the side of the limb corresponding to whichever of the hamstrings is to be transplanted, gives the best access; the vertical limb of the $J$ should be placed far back, and the lower end curved forward below the patella, allowing of the setraction of a flap of skin to give free access to the quadriceps tendon.

The hamstring must be freed high up the limb, so that there is no kinking in its line of pull. Its tendon should be threaded obliquely through the other, which is best split in the coronal plane, after it has been lifted by blunt dissection from the synovial membrane, so that that is not injured. The remarks regarding tension, made repeatedly above, apply to this as to all transplants. The knee must be held straight, and after the operation secured by some apparatus which allows no risk of displacement; probably a plaster cast applied (loosely over much wool) at the end of the operation is the most satisfactory. If the back half of the cast is strongly reinforced by folds of plaster bandage and the front left thin, then it can be split for removal of the stitches and the back half can be worn during the course of massage. Very few of the knee-splints in common use fit well enough not to get displaced, and if this happens on a single occasion, the whole operation may be undone, as the lower limb is heavy and exerts a great leverage.

\section{(6) Transplants for the Tibialis Anticus.}

When the tibialis anticus is cut or paralysed, the disability is a double one:

(a) Weakening of dorsiflexion of the ankle from loss of the most powerful of the muscles which have this action.

(b) Loss of one of the most powerful of the invertors of the foot, the only one which acts on the front part of the ferot. in front of the ' mid-tarsal' joint. 
The tibialis posticus, the other invertor of importance, although described by anatomists as having insertions into nearly all the bones of the foot, yet is found in practice to invert the back part of the foot only (the heel). It is also a plantar-flexor, so that when it alone is acting the patient is only able to invert the foot during plantar-flexion; whereas the tibialis anticus enables him to invert and dorsiflex simultaneously. The extensors of the toes, which also dorsiflex the ankle, act as evertors of the foot at the same time.

The muscles available for replacing the tibialis anticus are the extensor proprius hallucis and the peronei.

The action of the extensor of the big toe can ill be spared from that digit ; it is of course possible to suture the distal end of its tendon to the extensor longus or to the brevis digitorum, so as to avoid dropping of the big toe. In some cases the extensor hallucis is sufficiently powerful to replace the tibialis anticus. In others it is too weak.

As regards the peronei :

The longus is a plantar-flexor as well as evertor, so that its removal from these functions weakens the direct opposers of the tibialis anticus and so to a certain extent restores the balance of the foot, while it is a powerful muscle which can be made to take a course that converts it into a direct dorsiflexor. It apparently cannot be made to invert with the force of a normal tibialis anticus, possibly because the peroneus brevis cannot be dissociated from contraction with it, but nevertheless the result of a transplant is found to be dorsiflexion of the foot in the neutral position, which is valuable for walking.

The peroneus brevis is a less powerful evertor than the longus; it is possibly itself a weak dorsiflexor. It gives a satisfactory result when transplanted into the tibialis anticus, but the foot tends to be a little less balanced than when the longus is used. In many of the cases, one of the pair is itself too damaged for transplantation so that the operator has not the choice.

\section{Details of the Operation,}

Incision $\boldsymbol{J}$-shaped, the vertical limb running down over the peronei and the lower end curving forward across the front of the ankle to expose the tibialis anticus tendon at tle upper border of the annular ligament, at which level the transplant can be done into it.

The peroneus longus is found as a long tendon running down the surface of the fleshy brevis; it is freed down to the external annular ligament; if it is divided at the upper border of this, sufficient tendon will be obtained to reach to the tibialis anticus. The muscle must be well freed upwards, care being taken not to injure nerve twigs entering it, and also to see that the muscle is not kinked where it is made to cross 
the external intermuscular septum; a direct line of pull is sometimes a little difficult to attain.

The peroneus is passed through a slit in the tibialis anticus tendon and sutured while the foot is held in extreme dorsiflexion and inversion. This position must be maintained with great care, which, in the writer's opinion can only be done satisfactorily by a plaster-cast. The cast should be kept on three weeks to allow firm healing; then the stitches can be removed and massage and re-education begun. Either the cast or a strongly inverted tin-shoe must be worn continuously. When the patient begins to be able to dorsiflex the foot actively, he can be allowed to walk with a foot-drop iron fixed to his boot to keep the foot at right angles and also with an inside $\mathbf{T}$-strap to maintain the inversion. These should not be omitted until active dorsiflexion with the boot on is obtained, and then they should be discarded gradually, i.e. for increasing periods each day. If the transplant has been done with correct tension and aftertreatment is efficient, the result of the operation is good.

\section{(7) Tendon-slinging for External Popliteal Paralysis.}

Where there is complete paralysis of the external popliteal nerve, the sensory loss does not distress the patient, but the motor loss is most disabling. He has no power to dorsiflex the foot or toes, nor to evert the foot, nor to invert the front part of it. The plantar-flexors 1emain intact; also one invertor, the tibialis posticus, acting on the heel alone ; also the extensors of the interphalangeal joints of the toes (intrinsic muscles of the foot). In walking, the front of the foot and the toes droop and do not clear the ground, unless the whole foot is lifted by flexion of the knee.

Sufficiently powerful muscles are not available for transplantation to restcre these three lost functions. Two alternatives remain: to anchylose the ankle-joint in the dorsiflexed position ; or to sling up the foot by its tendons, or by artificial slings, such as silk ones.

Fixation of the ankle-joint does not overcome the dropping of the front part of the foot and of the toes, and it does take away spring from the foot. To tackle all the joints involved is a lengthy operation, involving much shock; and it is most difficult to control the several joints at once so as to ensure fixation in a good position.

As regards the use of silk tendons, these are unsuitable in cases where there is extensive scarring, and consequently latent sepsis in the part of the limb where they are to be inserted, as absolute asepsis and a deep bed are essential for success when such a foreign body is put into the tissues. These conditions are seldom available in the lesions due to gunshot-wounds.

The following operation in which the distal ends of the paralysed 
tendons are used to sling the foot up to the bones of the leg has given satisfactory results, provided certain conditions are carefully observed.

In the operation as originally performed only the tibialis anticus and peroneus longus were dealt with. It has been found, however, that it is

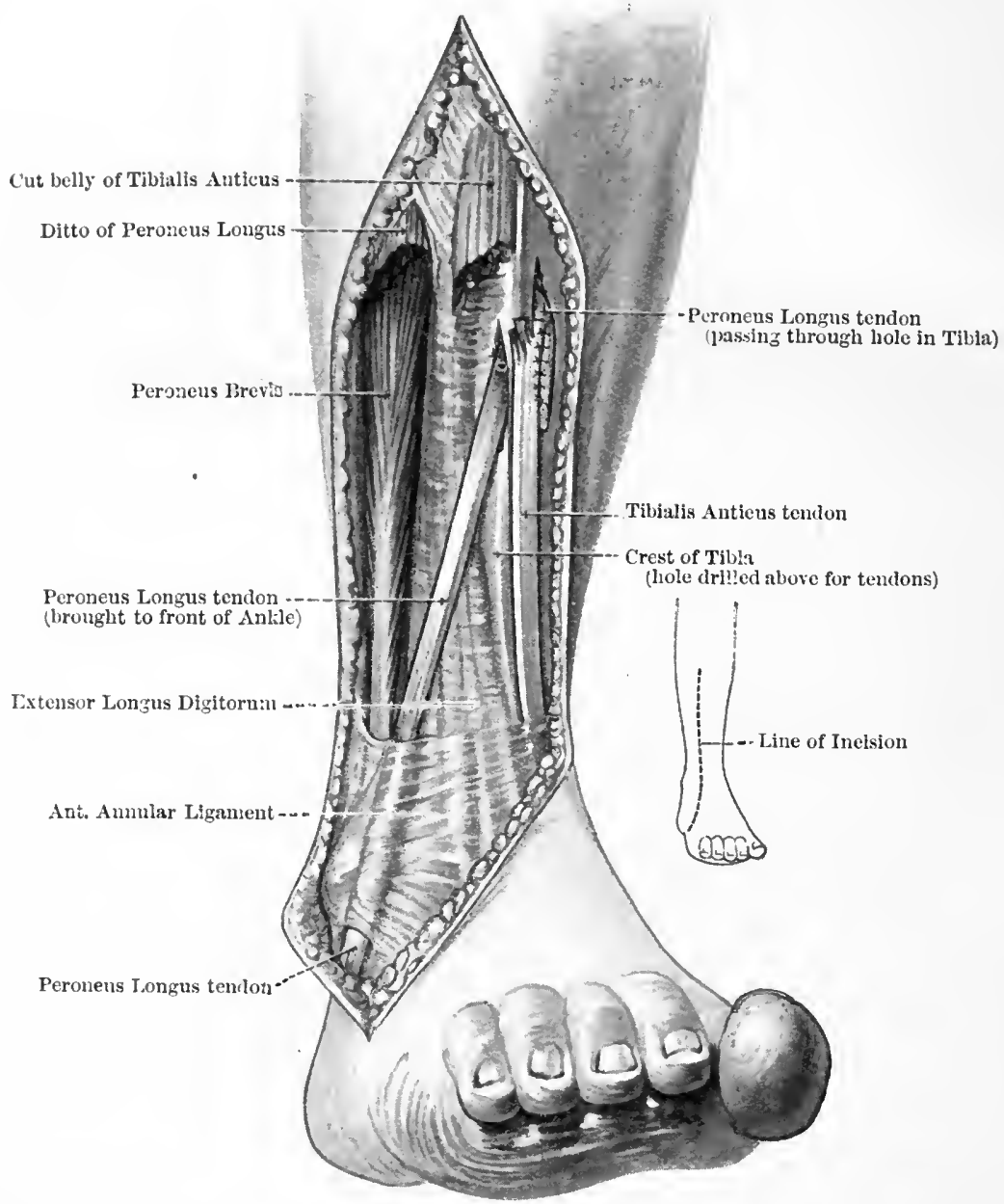

FIG. 58.-Tendon-slinging operation for foot-drop. The Peroneus Longus Tendon has been divided and brought forward out of its bed to the front of the limb, so as to pass up deep to the Anterior Annular Ligament and through a hole in the crest of the Tibia. The Tibialis Anticus tendon has also been detached from its origin and sutured to the free proximal end of the Peroneus, so that both act as slings for the foot.

important to sling up the toes also by the muscles inserted into them and also to use both peronei, because one invertor, the tibialis posticus, still remains acting unopposed. 


\section{Details of the Operation.}

Incision $\boldsymbol{J}$-shaped, the vertical limb running down midway between the tibia and fibula, and the lower end curving outwards below the external malleolus.

The outer flap of skin can be dissected up to give access to the peronei below the external annular ligament. The tibialis anticus tendon is freed upwards as high as possible and separated from the fleshy fibres; the same is done with the extensor proprius hallucis and extensor longus digitorum, so that all three tendons have a free upper cnd, while their distal insertion is not interfered with. Next the two peronei are exposed in the middle of the leg and similarly divided at the junction of their tendons with the fleshy bellies; their tendons are next exposed at the outer side of the foot and pulled out of the annular ligament, so that they can be made to run up the front of the ankle to the subcutaneous surface of the tibia.

Next a hole is made from the subcutaneous surface of the tibia through to its external surface, large enough for the tendons to be drawn through it. It is easily made with a narrow gouge by cutting obliquely from each surface in turn till the two openings meet. It is impossible to make a track large enough for all five tendons, but if possible the tibialis anticus and peroneus longus should both be made to pass through from opposite directions; the other tendons can later be sutured to them. When the two tendons have been passed through, they must be pulled very taut while the assistant keeps the foot in extreme dorsiflexion and neutral as regards inversion; then each tendon is secured by strong silk stitches to itself, so as to sling up the foot. It is attention to the position of the foot at this stage which makes or mars the operation. The tendons are apt to stretch a little in time, but if the foot has been dorsiflexed to $85^{\circ}$ at the operation will not slip below $95^{\circ}$ later, which gives a good result from the point of view of function. Some of the early cases done were unduly inverted, and one too much everted, which ultimately caused aching, as in any other type of flat foot. To obtain the correct angle is not easy.

When the two chief tendons have been fixed, the peroneus brevis and toe extensors can be sutured to them.

It is essential to fix the foot in plaster-of-Paris in the corrected position and to leave it, if possible, for six weeks. After that the patient should not walk without the support of a foot-drop iron for another six wceks and should wear a tin-shoe at nights, for it takes about three months for the tendons to be fixed by dense, unstretchable scar-tissue. The expenditure of this time in care will be a saving in the long run, for an unsuccessful operation leaves the foot in a very unsatisfactory 
state. In many cases the patient would prefer an amputation to a paralysed, inverted foot. In suitable cases, where there are no contractures, no spasm of the unopposed internal popliteal muscles, no trophic changes, and where the slinging operation has been carefully done, the patients have felt much benefit from it and been very pleased with the result.

Naturally these operations, where the distal end of a paralysed muscle is used as a passive sling, do not give a restoration of normal function comparable to that where an active muscle is made to act in some new manner. It must never be forgotten that stability rather than movement is of prime importance in the lower limb. Hence tendon-slingings have their place in it, whereas they seldom, if ever, are indicated in the upper limb.

Numerous other transplants are possible and useful in the lower limb, but they will not be dealt with individually, because the principles are the same in all and it is only. necessary to weigh in each case the number and quality of muscles available and the function which it is desired to restore.

The illustrations have been made by the artist from drawings done at the time of the operations by one of the authors (M.F.B.). 


\section{INDEX}

Abnormal distribution of nerves, 37 .

Adhesions of nerves, 44 .

After-treatment-nerves, 56, 63 .

transplants, 154 .

Anatomy of the nerves, 2-2I.

Aneurysm complicating nerve injury, 98 .

Anterior crural nerve-operation, 144.

Arterial lesions, 17,55 .

Artery, subclavian, 87 .

Atrophy-in arterial lesions, 17, fig. 7 . effects of massage on, 64 .

Axilla-nerve operations, 93.

Bed for nerves, 56, 77 .

for tendons, 152 .

Bone-shortening, 5o, I 47 .

Brachial plexus-structure of, 3 .

relations, 82 .

operations on, 84 .

transplantations for injuries of, I5I, fig. 48 .

Causalgia, I 48 .

Circumflex nerve-anatomy, 5, 7 . operation on, 95.

Clawing, II, 65 ; figs. 4 , I7.

transplant for, 167 .

Complications of nerve injuries, $5 \mathrm{I}, 54$, I +9 .

Constriction of a nerve, 44 .

Contractures, $65, I_{5}$.

Coraco-brachialis, 94.

Cords of brachial plexus, $\uparrow$.

Diagnosis-general principles, $2 \mathrm{I}$. special difficulties, 30 .

Electrical tests, 29, 39. at operation, 74 .

Extensor carpi radialis longior, use in transplantations, $165, \mathbf{I} 7 \mathbf{I}$.

Extensor paralysis, transplantations for, I 56 , I 7 I.

External popliteal nerve-anatomy, I8. operations on, 135.

tendon-slinging for paralysis of, 175 .

lilexor carpi radialis, use in transplantations, 161,171 .

lifexor carpi ulnaris, use in transplantations, $15 S$.

Flexor paralysis, transplantations for, $123,112,172$.

loot-nerve operations, $1+3$.
Gaps in nerves-possibilities of suture, 47.

Grafting of nerves, 5I, 56, I44.

Hamstrings, use in transplantations, 172.

Hand-nerve operations, 124 .

Incomplete lesions of nerves, operations for, $51,72,74$.

Indications for exploration of nerves, 4.5 . for tendon-transplants, 149 .

Induration of nerves, 44 .

Inner cord of brachial plexus, 5, 6 .

Internal popliteal nerve-anatomy, I9. operations on, 137 .

Interosseus paralysis, 35 .

Intrinsic hand muscles-actions, 34 . transplant for paralysis of, $\mathbf{I} 66$.

Irritation of nerves, 17 .

Joint-stiffness, 55, 66 . stretching after operations, 73 .

Lesions of nerves-types of, $4 \mathrm{I}$.

Lumbrical paralysis, 34 . transplantation for, 167 .

Median nerve-anatomy, II, operations on (axilla), 94, 99 ; (elbow), I 20 : (forearm), I I 7 .

transplantation for paralysis of, 123. 162 .

Motor branches, arrangement of, 79 .

Motor tests, 23, 31 .

Nuscle-substitution, 32.

Iusculo-cutaneous nerve, 5, $11,94$.

Musculo-spiral nerve-anatomy; 7 . operations on, IOO, IO2.

transplants for paralysis of, $15^{6}$.

Nerve-bed, 56.

Operations on the nerves-tachnigue, 70 .

Order of recovery, 57.

Origins of the limb nerves, $2-2 \mathrm{I}$.

Outer cord of brachial plexus, 5 .

Overstretching of muscles, 13.

Peripheral clistribution of the nerves. $2-21$.

Peronei, use in transplantations, $17 t$

l'hrenic nerve, s.3.

l'lantar nerves-anatomy, 20.

operations on, It.3. 
Pleura-accidental injury of, 89.

Posterior cord of brachial plexus, 4-6.

Posterior interosseus nerve-anatomy, 8 . operations on, 108.

Posterior tibial nerve-anatomy, I9. operations on, I39.

Prognosis of nerve injuries, 52.

Pronator radii teres-use in transplantations, 157 .

Pseudo-neuroma, 43.

Quadriceps-transplantation for paralysis of, 172 .

Re-education, 57 .

Regeneration, rate and order, 58 .

Results of nerve-grafting, $1_{4} 6$.

Sacrifice of nerve branches, 50 .

Sciatic-anatomy, I8.

operations on, 125 .

Sensory distribution $\mathrm{v}$. individual nerves.

Sensory tests, 3, 24, 26, 31 .

Sepsis, influence of, 52 .

Skin changes, 27.

Splints-for musculo-spiral injuries, fig. 49 .

for ulnar operations, figs. I $4, I_{7}$.

for sciatic operations, fig. I3.

Stiffness, v. joints.

Subclavian artery, 87 .

Substitution of muscles, 32 .

Suture of nerves across gaps, 47 .
Suture material for nerves, 56,76 . for transplants, I 53 .

Synergism of muscles, I53.

Technique of nerve operations, 70 . of tendon-transplants, ${ }_{5} \mathrm{O}$.

Tendon injuries, 55 .

Tendon-slinging operation on foot, 175 .

Tendon-transplantations, I 49 .

Tension after nerve-suture, 56 . tendon-transplants, 152 .

Thenar muscle paralysis, 35 . arthrodesis for, 123 , I66.

Tibialis anticus-transplant for paralysis of, 173 .

Tinel's sign, 57, $24 \mathrm{I}$.

Transposition of nerves, 49.

Trophic changes, 25, 27; and v. individual nerves.

in arterial lesions, $\mathbf{I} 7$.

Trunks of brachial plexus-upper one, exposure of, 85 .

lowest one, exposure of, 87 .

Types of nerve lesions, 4 . of operations, 46.

UInar nerve-anatomy, xo. operations on (axilla), 95, 100 ; (forearm), rog.

Vaso-motor phenomena in nerve injuries, 27.

Working capacity after nerve injuries, 67 . 


$4,3.0$

UC SOUTHERN REGIONAL LIBRARY FACILITY

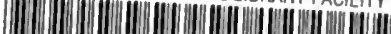

AA 000 (103633

AA $000103633 \quad 4$ 


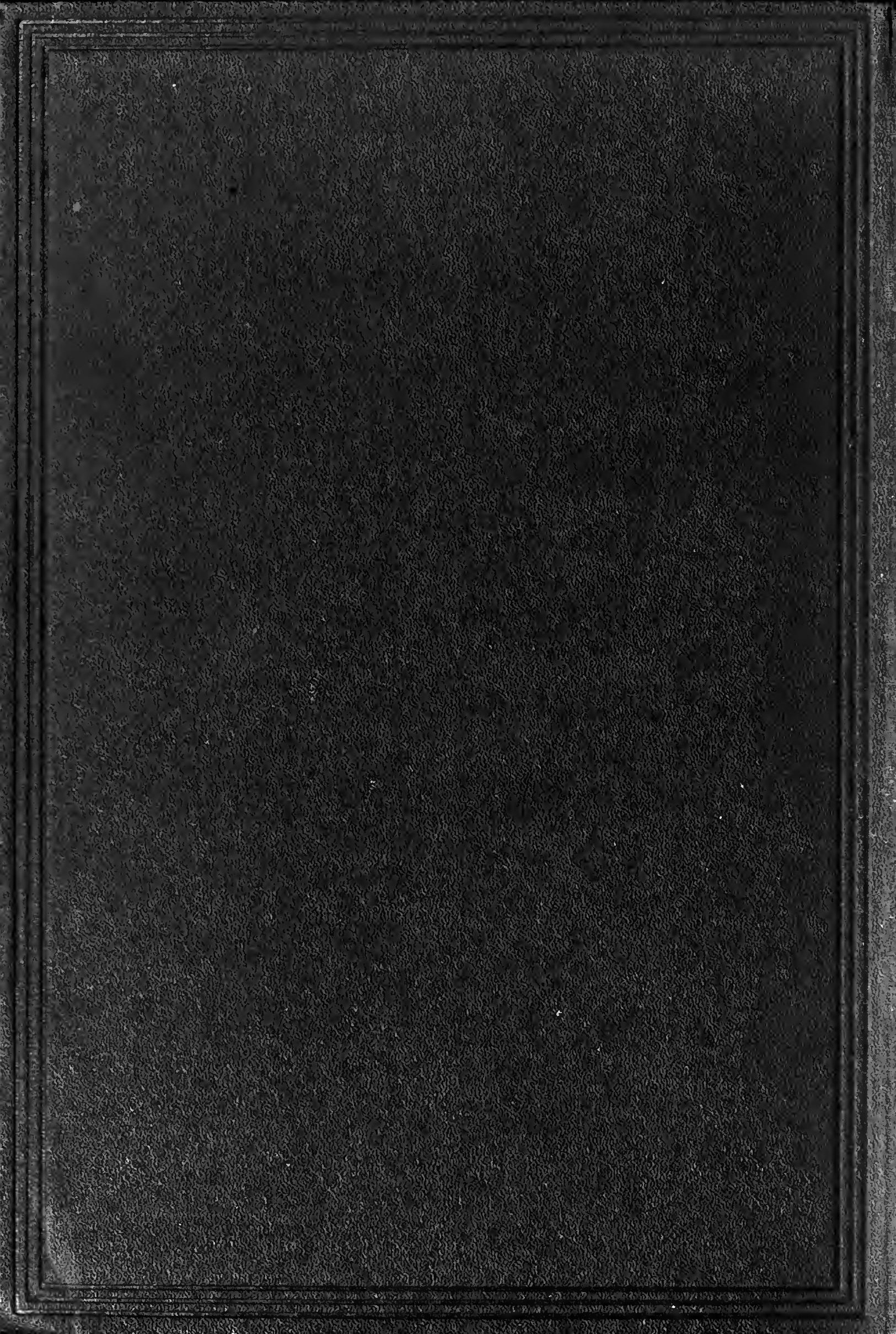

